ACCEPTED MANUSCRIPT • OPEN ACCESS

\title{
Pushing the boundaries of lithium battery research with atomistic modelling on different scales
}

To cite this article before publication: Lucy Morgan et al 2021 Prog. Energy in press https://doi.org/10.1088/2516-1083/ac3894

\section{Manuscript version: Accepted Manuscript}

Accepted Manuscript is "the version of the article accepted for publication including all changes made as a result of the peer review process, and which may also include the addition to the article by IOP Publishing of a header, an article ID, a cover sheet and/or an 'Accepted Manuscript' watermark, but excluding any other editing, typesetting or other changes made by IOP Publishing and/or its licensors"

This Accepted Manuscript is @ 2021 The Author(s). Published by IOP Publishing Ltd..

As the Version of Record of this article is going to be / has been published on a gold open access basis under a CC BY 3.0 licence, this Accepted Manuscript is available for reuse under a CC BY 3.0 licence immediately.

Everyone is permitted to use all or part of the original content in this article, provided that they adhere to all the terms of the licence https://creativecommons.org/licences/by/3.0

Although reasonable endeavours have been taken to obtain all necessary permissions from third parties to include their copyrighted content within this article, their full citation and copyright line may not be present in this Accepted Manuscript version. Before using any content from this article, please refer to the Version of Record on IOPscience once published for full citation and copyright details, as permissions may be required. All third party content is fully copyright protected and is not published on a gold open access basis under a CC BY licence, unless that is specifically stated in the figure caption in the Version of Record.

View the article online for updates and enhancements. 


\title{
Pushing the boundaries of lithium battery
}

\section{research with atomistic modelling on different}

\author{
scales
}

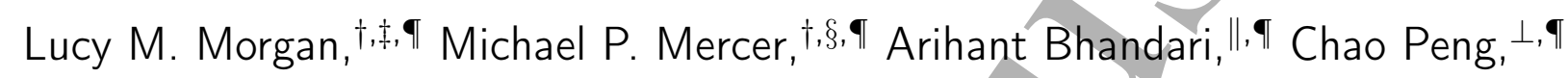
Mazharul M. Islam, Hui Yang, \#, Julian Holland, Sharpe ${ }^{\ddagger}$ Aron Walsh,, @, Benjamin J. Morgan, Denis Kramer, ${ }^{\perp, \triangle,}$ M. Saiful Islam, ${ }^{\ddagger}$ Harry E. Hoster, ${ }^{\S}$ Jacqueline Sophie Edge, ${ }^{*, \nabla, \Phi}$ and Chris-Kriton Skylaris*,

$\dagger$ The two authors contributed equally to this review.

$\ddagger$ Department of Chemistry, University of Bath, Claverton Down, Bath BA2 7AY, UK

\The Faraday Institution, Quad One, Harwell Campus, Didcot, OX11 0RA, UK

$\S$ Department of Chemistry, Lancaster University, Bailrigg, Lancaster, LA1 4YB, UK

\|School of Chemistry, University of Southampton, Southampton SO17 1BJ, UK

$\perp$ School of Engineering, University of Southampton, Southampton SO17 1BJ, UK

\# Department of Materials, Imperial College London, London SWr 2AZ, UK

@Department of Materials Science and Engineering, Yonsei University, Seoul 03722, Korea $\triangle$ Faculty of Mechanical Engineering, Helmut-Schmidt University, Holstenhofweg 85, 22043

Hamburg, Germany

$\nabla$ Department of Mechanical Engineering, Imperial College London, London, SW' 2AZ, UK E-mail: j.edge@imperial.ac.uk; c.skylaris@soton.ac.uk 
Abstract 


\title{
Contents
}

\author{
1 Introduction
}

2 Methods

2.1 Method overview . . . . . . . . . . . . . . . . . . . . . 14

2.1 .1 Density Functional Theory . . . . . . . . . . . . . . . . . . . . . . 14

2.1 .2 Linear-Scaling DFT . . . . . . . . . . . . . . . . . . 17

2.1 .3 Nudged Elastic Band . . . . . . . . . . . . . . . . . . . . . 20

$2.1 .4 \quad$ Cluster expansion . . . . . . . . . . . . . . . . . . . . . . . 21

2.1 .5 Lattice gas and Monte Carlo . . . . . . . . . . . . . . . . . . 23

2.1 .6 Molecular Dynamics . . . . . . . . . . . . . . . . . 25

2.2 Method Development . . . . . . . . . . . . . . . . . . . 28

2.2.1 Continuum models of electrolyte solutions within Density Functional Theory . . . . . . . . . . . . . . . . . . 28

$2.2 .2 \quad$ Fitting Potentials for Classical Molecular Dynamics . . . . . . . . . . 32

2.3 Calculating observable properties . . . . . . . . . . . . . . . . . . 34

2.3 .1 Equilibrium voltage . . . . . . . . . . . . . . . . . . . 34

2.3 .2 Activity coefficients of electrolytes . . . . . . . . . . . . . . . . . . 37

2.3 .3 Diffusion coefficients . . . . . . . . . . . . . . . . . . 37

2.3 .4 Vibrational and Thermal Properties . . . . . . . . . . . . . . . . . 40

\begin{tabular}{|ll|l}
\hline 3 Anodes & 41
\end{tabular}

3.1 Introduction . . . . . . . . . . . . . . . . . . . 41

3.2 Bulk Properties . . . . . . . . . . . . . . . . . . . . . . . . . . . . . . . . . 43

3.2 .1 Graphite structure and Li-graphite stages . . . . . . . . . . . . . . . 43

3.2 .2 Equilibrium potential and measured open circuit voltage . . . . . . . 44

3.2 .3 Entropy $\ldots \ldots \ldots$. . . . . . . . . . . . . . . . . . . . . . . 49

3.2 .4 Ion diffusion in Li-GIC . . . . . . . . . . . . . . . . . 53 
$3.3 \quad$ Graphite Surfaces and Interfaces . . . . . . . . . . . . . . . . 56

3.3.1 $\quad$ Possible graphite surfaces and their stability . . . . . . . . . . . 56

$3.3 .2 \quad$ Surface Effect on Intercalation Energy _ . . . . . . . . . . . . 57

3.3 .3 The Surface Effect on Li Diffusion . . . . . . . . . . . . . . . . . . 60

3.3 .4 Li deposition on graphite anodes . . . . . . . . . . . . . 63

3.3 .5 Solid-Electrolyte Interphase _. . . . . . . . . . . . . . . . . . . 64

$3.4 \mathrm{C} / \mathrm{Si}$ composities $\ldots \ldots \ldots \ldots \ldots \ldots$

3.5 Outlook and challenges for anodes . . . . . . . . . . . . . . 69

\begin{tabular}{|lll}
\hline 4 & Electrolytes & 72
\end{tabular}

4.1 Introduction . . . . . . . . . . . . . . . . . . . . 72

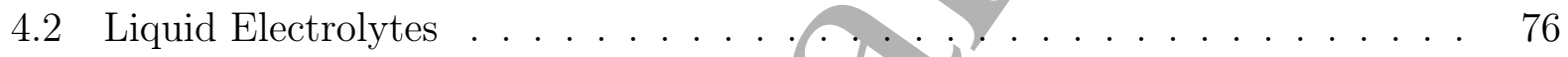

$4.2 .1 \quad$ Introduction to liquid electrolyte materials . . . . . . . . . . . . . 76

4.2 .2 An introduction to modelling liquid/electrolytes . . . . . . . . . . . . 77

$4.2 .3 \quad$ Ab initio modelling of liquid electrolytes . . . . . . . . . . . . . . . . 78

4.2 .4 Classical modelling of liquid electrolytes . . . . . . . . . . . . . . . . 81

4.2 .5 Bulk Structure and Landscaping . . . . . . . . . . . . . . . . . . . . . 83

4.2 .6 Li-ion Diffusion . . . . . . . . . . . . . . . . . . . . . . . . 85

4.2 .7 Solvation Energiès . . . . . . . . . . . . . . . . . . . . . . . . 88

4.2 .8 Activity coefficients of electrolytes . . . . . . . . . . . . . . . . . . . . 90

$4.2 .9 \quad$ Interfacial Nanostructure of Electrolytes $\ldots . . . . . . .90$

4.2 .10 Outlook and challenges . . . . . . . . . . . . . . . . . . 93

4.3 Solid Electrolytes $\quad \ldots \ldots \ldots 6$

4.3 .1 Introduction . . . . . . . . . . . . . . . . . . . . . . . . 96

4.3 .2 Sulfides . . . . . . . . . . . . . . . . . . . . . 99

4.3 .3 Oxides . . . . . . . . . . . . . . . . . . . . . . . 103

4.3 .4 Interface stability . . . . . . . . . . . . . . . . 108

4.3 .5 Outlook and challenges . . . . . . . . . . . . . . . . . 113 
715 Cathodes

725.1 Introduction . . 115

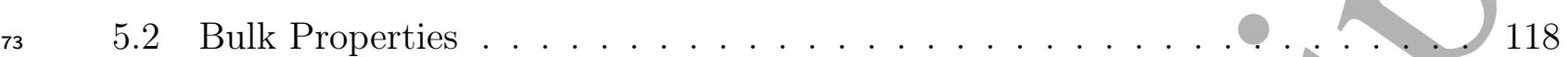

74 $5.2 .1 \quad$ Crystal Structure and Micro-Structure $\ldots \ldots \ldots . . . . .118$ 5.2 .2 Lithium-ion Diffusion . . . . . . . . . . . . . . . . . . . . 120

76 $5.2 .3 \quad$ Redox and Electronic Properties . . . . . . . . . . . . . . . . . . . . 124 5.2 .4 TM Ordering in NMC Layered Oxides . . . . . . . . . . . 127 $5.2 .5 \quad$ Vibrational and Thermal Properties . . . . . . . . . . . . . 128

83 Acknowledgement

${ }_{84}$ References 


\section{5 \\ 1 Introduction}

Lithium-ion (Li-ion) batteries (LiBs) were first commercialised by Sony in 1991. 1. They are ubiquitous in portable electronic devices, are emerging in hybrid and all-electric vehicles, 2 and are starting to play a role in large scale stationary storage. ${ }^{3}$ Despite over 30 years of commercialisation and longer for development, not all factors dictating their capacity, performance, safety, and longevity are completely understood. The complexity of battery systems makes it time consuming and impractical to directly measure all of their physical attributes. The grand challenge is to construct a multiscale model, incorporating inputs across lengthand time scales that can not only describe, but also predict, changes in behaviour.

To build a truly predictive modelling framework, a physical underpinning to battery models is required, incorporating physically correct descriptions of thermodynamic and kinetic battery behaviour. With sufficient accuracy built in, these models can provide insights on difficult-to-measure internal states, such as degree of Li intercalation and local electrolyte and ionic concentrations, as determined by the nanostructure of the materials used. By contrast, empirical models, which fit a curve to experimental data, are widely used in battery research, but have only a limited physical basis or, in some cases, no physical basis at all. For example, equivalent circuit models, which are widely used in industry, cannot be relied upon to predict battery behaviour over several charge-discharge cycles.

Physics-based continum models attempt to describe the behaviour of whole cells, for example the widely used Doyle-Fuller-Newman (DFN) model. 4 [ ${ }^{8}$ These models need to use drastic simplifications to enable them to run in real time, but their accuracy can be greatly improved by adopting parameters measured using more detailed, microscopic simulations. Atomistic models are key to building truly physics-based models and form the foundation of the multiscale modelling chain, leading to more robust and predictive models.

Atomistic models can also be applied to fundamental research questions with high predictive accuracy. For example, they can be used to predict new behaviour not currently accessible by experiment, for reasons of cost, safety, or throughput. They can be used to op- 
timise experimental design and use resources more efficiently, determining whether particular experiments are even worth performing and also provide unique insights into the behaviour of materials that may not even be accessible, or are impractical to obtain, by experimental probes. Atomistic models are useful for quantifying and evaluating trends in experimental data, explaining structure-property relationships and informing materials design strategies and libraries.

With the above in mind, we target this review to the following audiences. Non-atomistic battery modellers, such as continuum and control modellers, who would benefit from an easily accessible summary of atomistic methods and how they connect with longer length scale models. As atomistic models comprise a range of methods, it is also beneficial to summarise how these interlink, since it is possible to be an expert in one area, whilst being unfamiliar with another. For this reason, it is instructive to summarise these different methods in one place, so that non-specialists can understand these links. Recent developments in these techniques, such as linear scaling Density Functional Theory (DFT), require summarising standard DFT techniques. Likewise, longer length scale atomistic techniques can be parameterised using ab initio data. While DFT and other atomistic methods are widely known and utilised individually, their other applications and connections to one another are often not as thoroughly described, nor do these descriptions account for more recent developments.

This review also aims to reach those working in the battery area, such as experimentalists, who may not be experts in atomistic modelling and want to understand how atomistic models can help to understand experimental behaviour. Likewise, we show examples of behaviour currently out of reach with experiments. Similarly, this review is of benefit to junior researchers new to the battery area, and those who are already familiar with atomistic methods but are new to lithium batteries. With these audiences in mind, we have summarised applications of atomistic methods in lithium-ion batteries and all solid-state batteries (ASSBs), which represent the most technologically advanced rechargeable battery 

a $\mathrm{LiCO}_{2}$ cathode. $\frac{13}{13}$ The low conductivity of these transition metal oxides requires addition scales, from the level of electronic structure calculations through conventional and linearscaling DFT, to ab initio Molecular dynamics (MD) and on to longer length scale models, such as classical MD, Monte Carlo (MC), and kinetic Monte Carlo (kMC) calculations, which are parameterised by force field potentials or ab initio data. These techniques, along with recent method developments and battery-specific observable properties, are summarised in the methods section of this review, section 2.1. Specific applications to anodes, liquid and solid electrolytes, and cathodes are broken down in the following sections. Links between different methodologies are emphasised and this review may thus be of particular interest to those looking, for example, to link DFT calculations to MC calculations, or apply linear-scaling DFT to MD, bridging possible gaps in nomenclature at different length scales. Atomistic models linking to ab initio calculations are summarised by Van der Ven et al.;9 also noteworthy in this area is a review by Shi et al. ${ }^{10}$ and an older review by Franco. 11 A recent review of method development in the area of hybrid quantum-continuum solvation models is presented by Herbert. 12

The review covers mechanisms in both the conventional liquid electrolyte based and solidstate based LiB, as shown schematically in Figure 1. In a single cell of a conventional LiB, as shown here, the anode, or negative electrode, comprises a copper current collector and the primary active material is graphite in the vast majority of commercial LiBs. Increasingly, small amounts of silicon are being added to boost the gravimetric capacity. The electrode material also comprises a binder, such as polyvinylidene fluoride (PVDF), and sometimes a small fraction of conductive carbon is added to boost conductivity. The two electrodes are divided by a separator soaked in an organic electrolyte, which is usually a mixture of carbonates with dissolved $\mathrm{LiPF}_{6}$ salt. The cathode, or the positive electrode, has an aluminium current collector. Various different types of cathode material are utilised in commercial LiBs, with the example shown here being the classic "rocking-chair" battery with

The family of atomistic models itself represents a range of different length- and time 


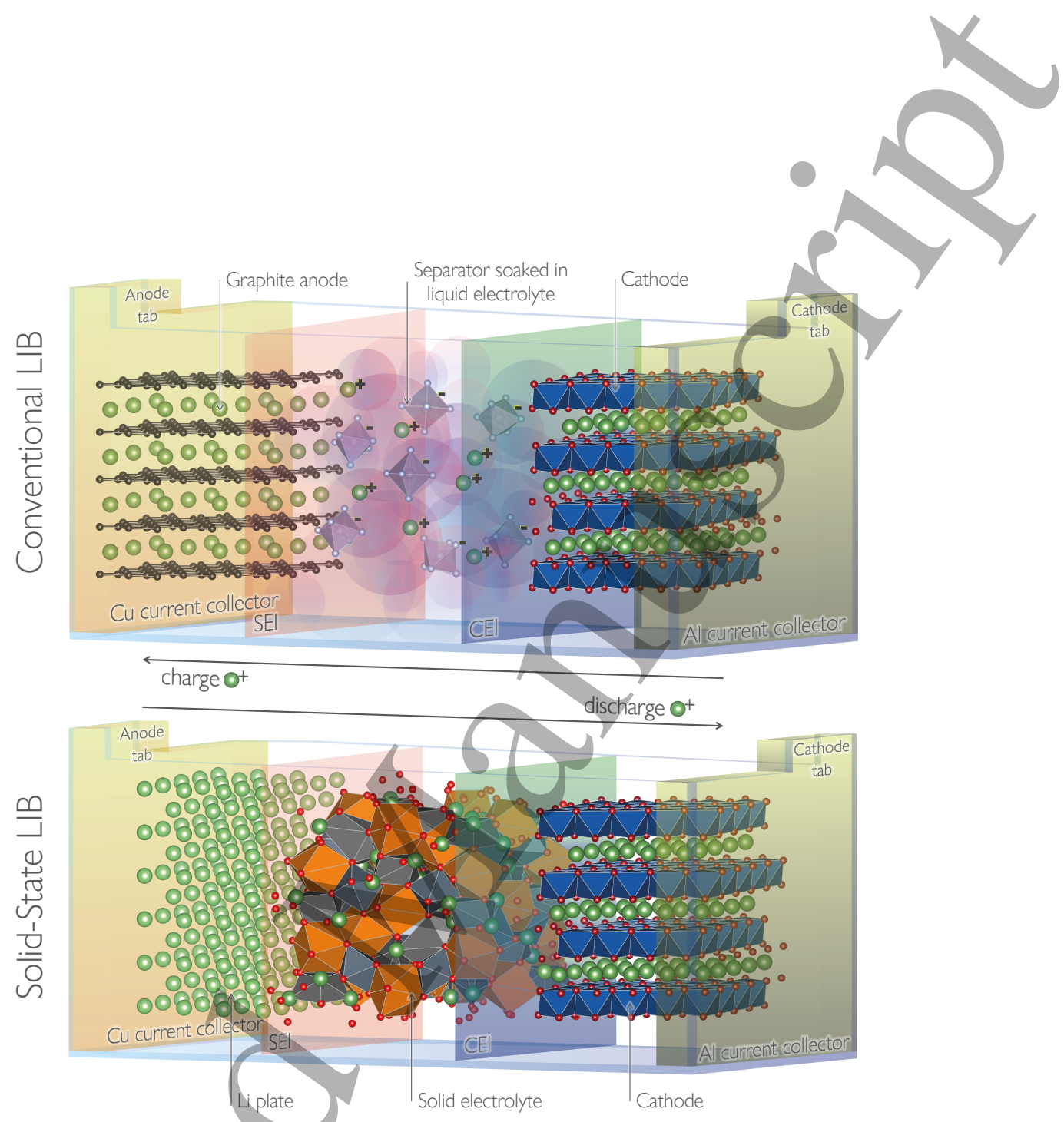

Figure 1: A schematic of a single cell of a conventional, liquid-based lithium-ion battery (LiB) and a solid-state $\mathrm{LiB}$. The conventional $\mathrm{LiB}$ comprises an anode composed of a $\mathrm{Cu}$ current collector and an active anode material (graphite), a separator soaked in an organic electrolyte, and a cathode composed of a Al current collector and an active cathode material, for example, $\mathrm{LiCO}_{2}$, as shown here. The solid-state $\mathrm{LiB}$ comprises a similar cathode, a solid electrolyte, and an anode composed of a Li-ion plate and $\mathrm{Cu}$ current collector. The anode-electrolyte interphase (SEI) and cathode-electrolyte interphase (CEI) for both LiBs are represented as pink and blue transparent layers, respectively. The tabs are shown protruding from the top of the current collectors. Both LiB cells show all components as fully lithiated, with directional $\mathrm{Li}^{+}$movement during (dis)charge indicated with arrows. 

of conductive carbon and, as with the anode material, the active cathode material is held together with a binder, such as PVDF. When the cell is assembled, the cathode starts fully lithiated and the anode is completely delithiated. On the first cell charge cycle (also known as the formation cycle) lithium is removed from the cathode and the anode becomes filled with lithium while the solid electrolyte interphase (SEI) and cathode electrolyte interphase (CEI) are formed. While Figure 1 shows both electrodes in a fully lithiated state, Li is transferred between the electrodes reversibly during (dis)charging, therefore allowing this system to be rechargeable.

Although not yet commercialised, ASSBs are a promising future alternative to conventional liquid electrolyte LiBs. Their anode, or negative electrode, comprises a copper current collector and either a a metallic lithium plate (Li-metal), as shown in Figure 1, or less commonly a graphite-based material (Li-ion). As there is no liquid, there is no longer a need for separators, with the two electrodes being separated by the solid electrolyte material, shown here with $\mathrm{Li}_{7} \mathrm{La}_{3} \mathrm{Zr}_{2} \mathrm{O}_{2}$ (LLZO). The cathode, or positive electrode, has an aluminium current collector and, as with the conventional LiB, can accommodate various cathode materials, such as $\mathrm{LiCO}_{2}$. The interfacial regions between the electrodes and the solid electrolyte are known as the solid-solid interphase,/or anode/cathode-solid interphase. Figure 1 shows both electrodes in a fully lithiated state; however, the Li is transferred between the electrodes reversibly, as in conventional LiBs.

The anodes section, section 3 , heavily focuses on graphite, which is still the predominant anode material in Li-ion cells. The section describes atomistic modelling of bulk graphite, graphite edges where initial Li-ion insertion occurs, and the Solid-Electrolyte Interphase (SEI). The bulk modelling discussion includes a direct comparison between experimental and theoretical thermodynamic parameters, such as the open circuit voltage (OCV) and entropy, which will also be of interest to battery control modellers. Kinetic predictions are made and linked to DFT predictions of the influence of graphite edge morphology on surface states, which may be of interest to those working on battery material development 

and discovery. Recent work applying linear scaling DFT to complex interfaces will be of interest to those at the forefront of DFT method development, focusing on the boundary between atomistic and continuum modelling. Lastly, recent developments in silicides to boost anode gravimetric capacity, along with their associated challenges, are summarised in the outlook. Recent reviews in this area include Asenbauer et al., ${ }^{14}$ summarising aspects of lithiation/delithiation mechanisms and morphological aspects in graphite and silicon oxide composites, and Zhang et al. ${ }^{15}$ similar in scope but providing a more ab initio focus. Here, our review here covers graphite structure and lithiation/delithiation mechanisms, including surfaces and interfaces, which have tended to be neglected, although aspects of modelling the SEI have been reviewed by Wang et al. 16

The liquid electrolyte section, section 4.2 , has a strong focus on the development of atomistic models, both $a b$ initio and force field-based. This includes a pivotal discussion on the atomic interactions between the components and method development to study electrolytes via classical MD simulations. This will be of particular interest to those at the forefront of classical MD method development. Liquid electrolytes are known to be limited by narrow electrochemical windows, solyent toxicity, and material flammability/safety concerns. The latter parts of this section describe the atomistic modelling of the bulk structure and landscaping, Li-ion diffusion, solvation energies, and activity coefficients of liquid electrolytes, and the interfacial nanostructure relating to the interface with a solid electrode. These topics cover the major aspects for improving liquid electrolytes for use in a battery and research towards circumventing critical safety ${ }^{17 \mid 18}$ and energy density ${ }^{19}$ limitations. The challenges and potential avenues for solving these issues are summarised in the outlook, including recent developments to resolve these within the liquid electrolyte family and alternative materials. Recent reviews in this area include Galiński et al., ${ }^{20}$ summarising the field of ionic liquids, Wang et al., ${ }^{21}$ reviewing the recent progress in water in salts electrolytes, and Logan and Dahn, 22 giving some recent developments in conventional electrolytes. Here, our review ${ }_{219}$ covers the continued development of interatomic potentials for liquid electrolytes and a de- 

scription of the solid electrode-liquid electrolyte interface from the perspective of the liquid, which is not the conventional frame of reference.

Solid state electrolytes (SSEs) are becoming an increasingly popular avenue of research, motivated by the rise of the electric vehicle (EV). $\stackrel{23}{23}$ They have been proposed as an alternative to liquid electrolytes to resolve safety issues pertaining to the flammable organic liquid electrolytes that are currently used, $\stackrel{17 / 18}{1}$ and also as a route to increased energy density. 19 In the solid electrolyte section, section 4.3 , we review a selection of the promising candidate materials currently being investigated. Each material discussed has a different focus, highlighting a range of properties applicable to different SSE materials. In this section, we focus on four material families, grouping them into sulfide and oxide based SSEs. Sulfide based SSEs typically have a high Li-ion conductivity and poor electrochemical stability against $\mathrm{Li}$ metal (the anode typically used in combination with $\mathrm{SSEs}$ ) ${ }^{24 / 25} \mathrm{Li}_{10} \mathrm{GeP}_{2} \mathrm{~S}_{12}$ (LGPS) is reviewed, with a focus on how atomistic methods reveal the isotropic ion pathways, while $\mathrm{Li}_{6} \mathrm{PS}_{5} X$ based Li-argyrodites are focused towards the relationship between ionic conductivity and anion substitution, as well as atomistic predictions of occupied Li sites. Oxides typically have a higher electrochemical stability but still suffer from dendrite formation, amongst other issues. $\frac{24}{2 L Z O}$ is also reviewed, with a focus on how multiple atomistic methods have been applied to probe dendrite formation and ionic transport in the material. State-of-the-art models of interfaces in oxide nanocomposites are reviewed. Lastly, the challenges of the SEI are discussed and an outlook to future modelling of SSEs is given. Related reviews in the area include Zhang et al. ${ }^{26}$ summarising the future directions of ASSBs, and Gurung et al., ${ }^{27}$ highlighting the advances and challenges in SSEs and ASSBs. Xiao et al. ${ }^{28}$ and others ${ }^{29130}$ provide a more specific review of the SEI. Ceder et al. ${ }^{31}$ outlines the principles that should be employed when modelling SSEs. Here, our review discusses a broad range of SSE properties, following the notion that these properties are applicable to range of materials.

The cathodes section, section 5, covers a range of different cathode materials used in a 
variety of Li-ion cells. This section describes atomistic modelling in the bulk, at the surfaces, and the Cathode-Electrolyte Interphase (CEI). In discussing bulk modelling, a comparison of the different cathode crystal structures, micro-structuring, and available diffusion pathways within the material are covered, as well as important properties, including redox and electronic properties, transition metal ordering, and vibrational and thermal properties. Use of electronic structure methods modelling techniques has been essential for investigating crystalline structure, so will be of great interest to those who utilise DFT in their research. Surface structures and morphologies of cathode particles can be difficult to determine using experimental methods alone, which is where ab initio and potentials-based MD can provide vital insight. As with the SEI, linear-scaling DFT has recently been applied to CEI, where discussions on CEI will be of interest to those doing state-of-the-art DFT method development. Related reviews in the area include $\mathrm{Ma}, 32$ summarising modelling Li-ion battery cathode materials, Yan et al. 33 focusing on DFT calculations of cathode materials, and Wang et al. 34 discussing closing the gap between theoretical and practical capacities in layered oxide cathode materials. Our review includes a discussion on the CEI, which has recently been reviewed by Maleki Kheimeh Sari and Li. ${ }^{35}$ Here, our review covers thermal, electronic, dynamic, and structural properties for a range of prominent cathode materials in terms of both electronic structure methods and potential-based modelling, which have tended to be more isolated in other reviews.

Finally, we provide an outlook on the key remaining challenges for atomistic modelling of LiBs and promising future directions for resolving them. 
268

269

270

271

272

273

274

275

\section{Methods}

\subsection{Method overview}

\subsubsection{Density Functional Theory}

Density Functional Theory (DFT) is amongst the most accurate methods for atomistic simulations of materials, as it is a quantum mechanical method. This means that it is able to simulate the electrons in materials and how they result in all the observable processes and properties of a material. As electrons are microscopic particles, to simulate their properties we need to use the theory of quantum mechanics. However, the computational cost of calculations with this theory is very high, as all the observable properties are obtained from the wave function: a highly complicated function of many variables (proportional to the number of particles we are simulating) and, for exact solution, the computational effort scales exponentially with the number of particles. Approximate wave function based theories with more favourable computational scaling ( $\operatorname{such}$ as $\sim N_{e}^{5}$ or $\sim N_{e}^{7}$, where $N_{e}$ is the number of electrons in the calculation) have been developed, but the computational effort is still so high that they cannot be applied to molecules with more than a few atoms.

DFT is a reformulation of quantum electronic structure theory, where the central quantity is no longer the wave function, but instead the electronic density, $\rho(\mathbf{r})$, which is a comparatively simpler function of only one position variable, r. As a result, DFT has lower computational scaling, allowing simulations of much larger systems (up to a few hundred atoms on supercomputers). Another advantage of DFT is that it is formally an exact theory. Due to these two significant advantages, DFT is today the method of choice for most simulations.

DFT was originally developed by Hohenberg and Kohn $\stackrel{36 \mid 37}{37}$ and reformulated by Kohn and Sham ${ }^{38}$ into the mathematical description we use today, often called KS-DFT, where the energy of a material is expressed as: 


$$
E[\rho]=T_{K S}[\rho]+E_{\text {ext }}[\rho]+E_{H}[\rho]+E_{x c}[\rho] .
$$

Here all the terms are expressed as functionals of the density and $T_{K S}[\rho]$ is the kinetic energy of the electrons, $E_{\text {ext }}[\rho]$ is the energy of attraction of the electrons to nuclei (also called external potential energy), $E_{H}[\rho]$ is the classical (Coulomb) electrostatic energy of the electronic density charge distribution (also called Hartree energy), and $E_{x c}$ describes the purely quantum effects of exchange and correlation.

DFT calculations are performed in an iterative fashion, with electron density expressed as a sum of one-electron wave functions, $\left\{\psi_{i}\right\}$, called molecular orbitals (MOs):

$$
\rho(\mathbf{r})=\sum_{i=1}^{N_{e}}\left|\psi_{i}(\mathbf{r})\right|
$$

and these MOs are obtained by solving the Kohn-Sham eigenvalue equation:

$$
\left[-\frac{1}{2} \nabla^{2}+v_{\text {ext }}(\mathbf{r})+v_{\mathrm{H}}[\rho](\mathbf{r})+v_{\mathrm{xc}}[\rho](\mathbf{r})\right]=\varepsilon_{i} \psi_{i}(\mathbf{r}) .
$$

As we can see from eqn. 3, the Hartree, $v_{\mathrm{H}}[\rho]$, and exchange-correlation, $v_{\mathrm{xc}}[\rho]$, potentials are functionals of the density, thus ultimately functionals of the MOs, which provide the solutions of the equation. This equation cannot be solved directly, but must follow an iterative procedure called the self-consistent field (SCF) process. The simplest SCF method is to guess a set of $\left\{\psi_{i}\right\}$ and use these to build and solve (eqn. 3), obtaining a new set of $\left\{\psi_{i}\right\}$ and repeating this process until the $\left\{\psi_{i}\right\}$ and the energy (eqn. 1) no longer change.

KS-DFT is formally an exact theory, but it does not provide an explicit expression for the exchange-correlation energy, $E_{\mathrm{xc}}[\rho]$. The exact exchange-correlation functional is unknown or, more precisely, unknowable. Thus a very active area of DFT development is to construct approximations of increasing accuracy for $E_{\mathrm{xc}}[\rho]$. The simplest approximation is the local density approximation (LDA), where $E_{x c}[\rho(\mathbf{r})]$ is expressed as: 


$$
E_{x c}^{L D A}[\rho(\mathbf{r})]=\int \rho(\mathbf{r}) \epsilon_{\mathrm{xc}}[\rho(\mathbf{r})] d \mathbf{r}
$$

The value of $\epsilon_{\mathrm{xc}}$ at some position, $\mathbf{r}$, is computed exclusively from the value of $\rho$ at that position. In practice, $\epsilon_{\mathrm{xc}}[\rho(\mathbf{r})]$ describes the exchange and correlation energy per particle of a uniform electron gas of density $\rho . \underline{39}$

In general, the electron density in a molecular system is not spatially uniform, even at small volumes of space, limiting the applicability of LDA. More accurate functionals are obtained by the inclusion of a density gradient correction, known as the generalised gradient approximation (GGA), or semi-local functionals. In the GGA, the functionals depend on both the density and the gradient of the density, i.e. $v_{x c}^{\mathrm{GGA}}=f(\rho, \nabla \rho)$. Popular examples of GGA functionals are Perdew-Wang GGA (PWGGA) (both exchange and correlation), 40 Perdew-Burke-Ernzerhof GGA (PBEGGA), ${ }^{41}$ and Becke-Lee-Yang-Parr (BLYP). ${ }^{42143}$ Functionals including contributions from the second derivative of the density are called meta-GGA functionals. 44

Standard DFT methods fail to describe dispersion effects that are of a non-local electron correlation nature. Consequently, DFT methods are often inaccurate for the investigation of molecular crystals, adsorption on surfaces, and other systems in which dispersion forces due to van der Waals (vdW) gaps between layers play a significant role. Several versions of dispersion corrected DFT (DFT-D) approaches are available, e.g. DFT-D2, ${ }^{45}$ DFT-D3, 46 DFT-D4, $\stackrel{47}{ }$ DFT-D3BJ,, 48149 etc.

GGA functionals, however, still have problems with self interaction. The hybrid functionals usually offer some improvement over the corresponding pure DFT functionals. Of all modern functionals, the B3LYP method is the most popular to date. ${ }^{43 \mid 50}$ It works well both for structural investigations and for the computation of electronic properties. ${ }^{51}$ An-

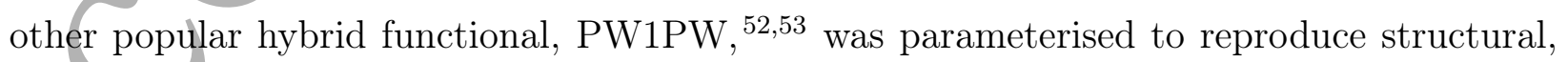
energetic, and electronic properties of solids. A more recent and popular hybrid functional is HSE06, where the correlation part is defined by a PBE functional and a range-separation 
approach is used for the exchange part. $\underline{54}$

The applicability of the hybrid functionals depends mainly on the type, size, and complexity of the studied systems, as these functionals incur a huge computational cost. An alternative approach is the DFT $+\mathrm{U}$ method, where the effects of strong intra-atomic electronic correlations are modelled by adding an on-site Coulomb repulsion, $U$, and site exchange term, $J$, to the DFT Hamiltonian. ${ }^{55-57}$ Parameters $U$ and $J$ can be extracted from ab initio calculations, but are usually obtained semi-empirically. Inspired by the Hubbard model, the DFT $+\mathrm{U}$ method is formulated to improve the ground state description of strongly correlated systems. The Hubbard Hamiltonian describes the strongly correlated electronic states ( $\mathrm{d}$ and $\mathrm{f}$ orbitals), while the rest of the valence electrons are treated by normal DFT approximations.

\subsubsection{Linear-Scaling DFT}

In conventional DFT, solving the Kohn-Sham eigenvalue equations, eqn. 3, subject to the required orthonormality constraint, results in a computational cost scaling with the third power (it is an $\mathcal{O}\left(N^{3}\right)$ procedure) with the number of atoms, $N$. This is demonstrated in the example of Figure 2, showing the computation time as a function of the number of atoms for slabs of graphite of increasing size. This unfavourable scaling is the reason why conventional KS-DFT is practically unfeasible beyond several hundred atoms. However, there are many grand challenges in materials research, where, due to their inherent complexity, building realistic models requires thousands of atoms, such as simulations of defects, complex structures of the Solid-Electrolyte Interphase (SEI), and metallic and semiconductor nanoparticles used in catalysis and battery electrodes, among others. This need for large-scale DFT calculations has motivated the development of new theoretical methods which can scale linearly with system size. ${ }^{58}$ In these linear-scaling methods, conventional KS-DFT is reformulated in terms of the one-particle density matrix, $\gamma$ : 


$$
\gamma\left(\mathbf{r}, \mathbf{r}^{\prime}\right)=\sum_{i} f_{i} \psi_{i}(\mathbf{r}) \psi_{i}^{*}\left(\mathbf{r}^{\prime}\right)
$$
can be clearly seen. DFT calculations with tens of thousands of atoms can be performed the density matrix decays exponentially with the distance, $\left|\mathbf{r}-\mathbf{r}^{\prime}\right|, \frac{59}{5}$ wile the MOs, $\left\{\psi_{i}\right\}$, are, in general, fully delocalised over the entire electronic system (molecule, nanoparticle, slab, etc.) and do not decay. The exponentially-decaying tail of the density matrix can be truncated to develop methods with reduced or linear-scaling computational cost. As the system size (number of atoms) is increased, it reaches a point where the remaining amount of information increases linearly with the size of the system. This can be implemented more efficiently with non-orthogonal, localised orbitals, $\left\{\phi_{\alpha}\right\} \cdot{ }^{60161}$ In this representation, the density matrix can be written as:

$$
\gamma\left(\mathbf{r}, \mathbf{r}^{\prime}\right)=\phi_{\alpha}(\mathbf{r}) K^{\alpha \beta} \phi_{\beta}^{*}\left(\mathbf{r}^{\prime}\right)
$$

Here, the density kernel matrix, $\mathbf{K}$, is a generalisation of the MO occupancies, $\left\{f_{i}\right\}$, of equation 5, while implicit summation (Einstein convention) is assumed for repeated Greek indices.

The development of linear-scaling methods has proven to be a very challenging research topic, as the goal of developing methods that accommodate the conflicting requirements of orbital localisation with high accuracy is extremely difficult to achieve. Recent developments towards this goal have made this possible by using a dual resolution approach, where both $\left\{\phi_{\alpha}\right\}$ and $\mathbf{K}$ are optimised self-consistently during the calculation, while subject to localisation constraints. ${ }^{62 / 64}$ The $\mathcal{O}(N)$ Electronic Total Energy Package (ONETEP), $\frac{65}{6}$ has the unique capability of achieving linear-scaling computational cost, while maintaining the near-complete basis set accuracy of conventional DFT. The computational efficiency of this code is demonstrated on the graphite example in Figure 2, where the linear-scaling behaviour

allowing us to exploit the principle of "nearsightedness of electronic matter", 59 because 
with ONETEP, opening avenues for simulating realistic models of materials and interfaces in lithium-ion batteries (LiBs) with DFT-scale accuracy. ONETEP is being actively developed and offers a large and diverse range of capabilities, including: different boundary conditions, various exchange-correlation functionals, finite electronic temperature methods for metallic systems, methods for strongly correlated systems, molecular dynamics, vibrational calculations, time-dependent DFT, electronic transport, core loss spectroscopy, implicit solvation, density of states calculations, and distributed multipole analysis. ${ }^{65}$ Recent focus in ONETEP is on developing specific electrochemistry tools for battery simulations, aiming to develop the first atomistic simulation platform (in particular, the first linear-scaling DFT platform) for electrochemistry. Some of these developments are described in this review, in subsection 2.2.1.

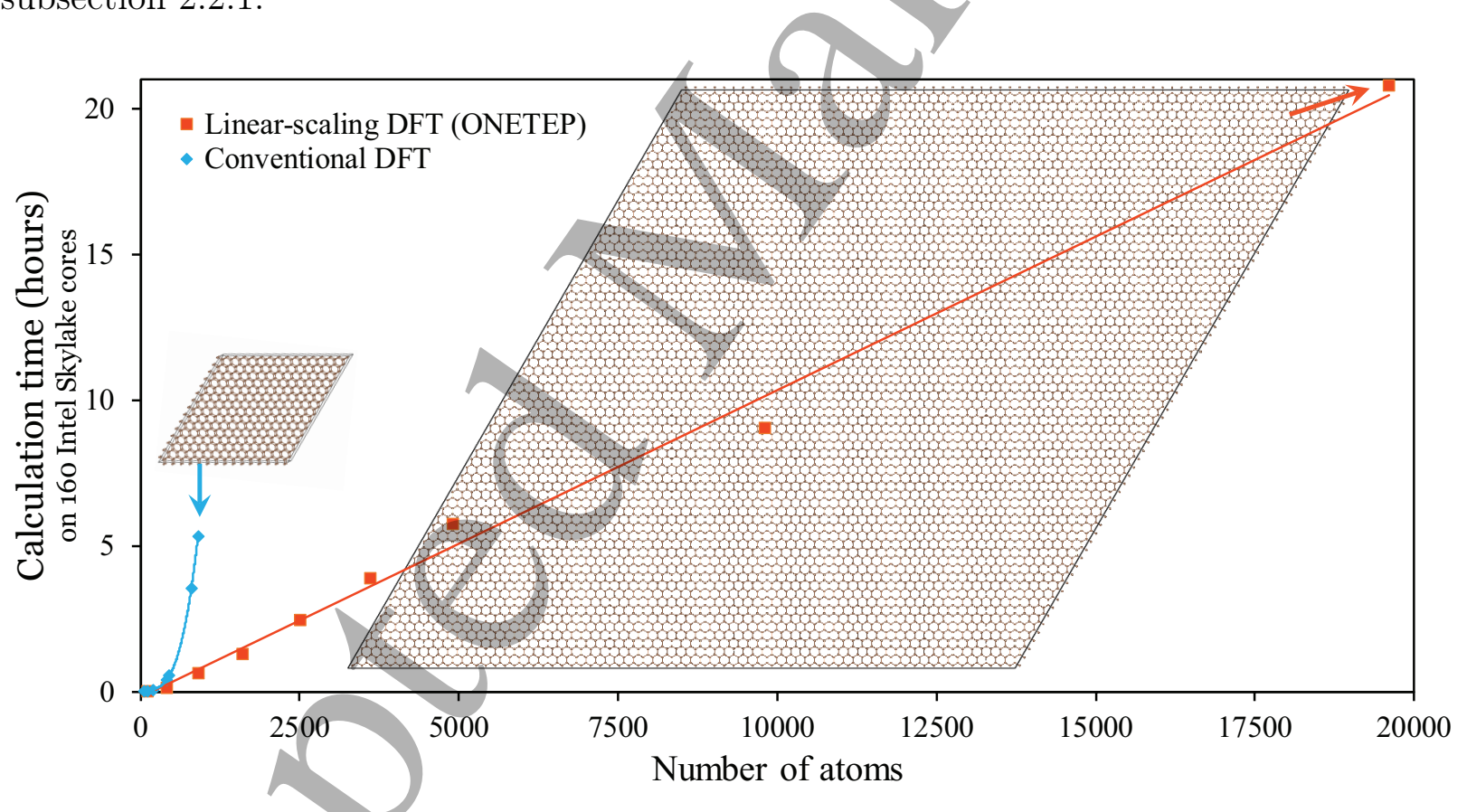

Figure 2: Comparison of the computational time with the number of atoms for slabs of graphite of increasing size using the ONETEP linear-scaling DFT code versus a conventional plane wave DFT code. The computations were performed on the Iridis 5 supercomputer at the University of Southampton on 40 MPI processes, with 4 OpenMP threads each (160 cores in total). Reprinted from Ref. 66, with the permission of AIP Publishing. 


\subsubsection{Nudged Elastic Band}

Nudged elastic band (NEB) theory is a useful method based on transition state theory, seeking the minimum energy path and the saddle point (or transition state) between two

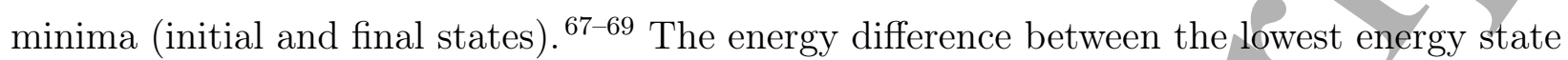
and the saddle point is defined as the activation barrier $\left(E_{a}\right)$, Figure. 3.69

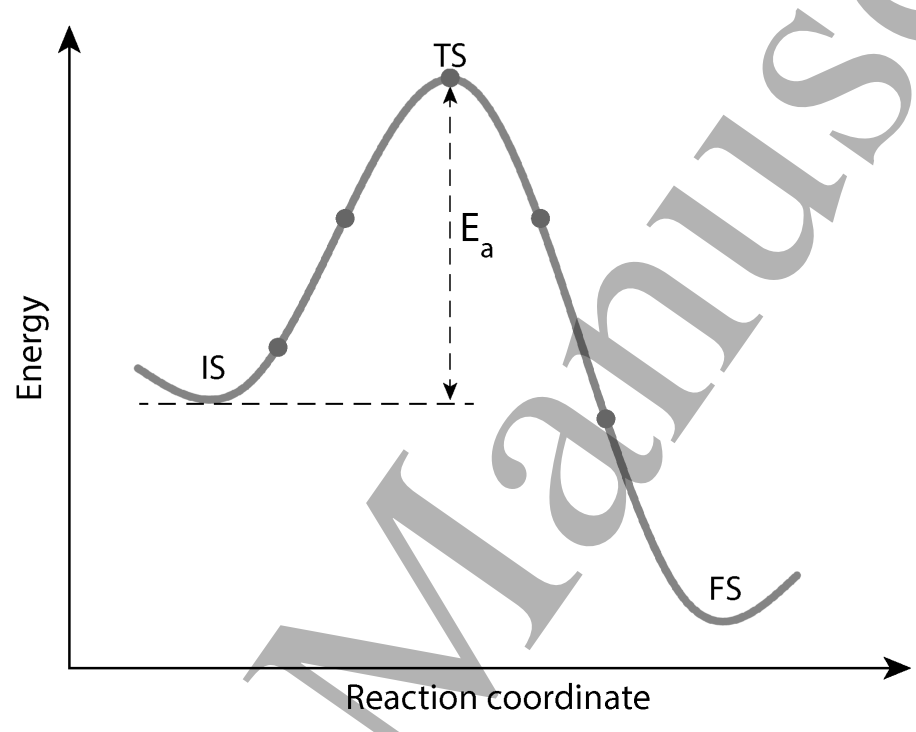

Figure 3: Energy profile of Nudged Elastic Band (NEB) calculation. The IS, TS, and FS are the initial state, transition state and final state, respectively. $E_{a}$ denotes the activation barrier along the reaction path. The grey circles are the "images" in the NEB calculation.

The NEB approach initially guesses a number of configurations of several possible intermediate "images" that may occur along the reaction coordinate or diffusion path. This set of images can be created by linear interpolation between the initial and final states. The NEB algorithm further conducts constrained optimisation and converges those images along the minimum energy path. Furthermore, fictional spring forces are added between adjacent images to maintain the spacing and the continuity of the reaction or diffusion path. The NEB approach is widely applied in the studies of chemical transformations, such as catalytic reactions or ion diffusion in solid materials. The determined chemical reaction energy barriers can then be used in further, larger time- and length-scale models, such as microkinetic models. $70 \mid 71$ 
410

411

412

413

414

415

416

\subsubsection{Cluster expansion}

The cluster expansion method enables a statistical approach to sample configurational phase space at finite temperature. ${ }^{72}+74$ This method aims to capture the energetics of mixing two or more atoms on a given set of lattice sites, typically with an accuracy close to DFT calculations. The approach borrows ideas from the Ising model, ${ }^{75}$ where each lattice site is assigned as a spin variable to simulate the magnetic properties, but maps site occupancy onto spin variables instead. ${ }^{76}$ For example, for a binary alloy system with atom types $\mathrm{A}$ and B, the occupation of each site can be described by a spin-like variable, i.e. $\sigma_{i}=+1$, if the site is occupied by atom $\mathrm{A}$, and $\sigma_{i}=-1$ if the site is occupied by atom $\mathrm{B}$, as shown in Figure 4 A configuration can then be written as $\sigma=\left(\sigma_{1}, \ldots, \sigma_{n}\right)$. Accordingly, the energy of each configuration can be expressed as: $E \equiv E\left(\sigma_{1}, \ldots, \sigma_{n}\right)$.

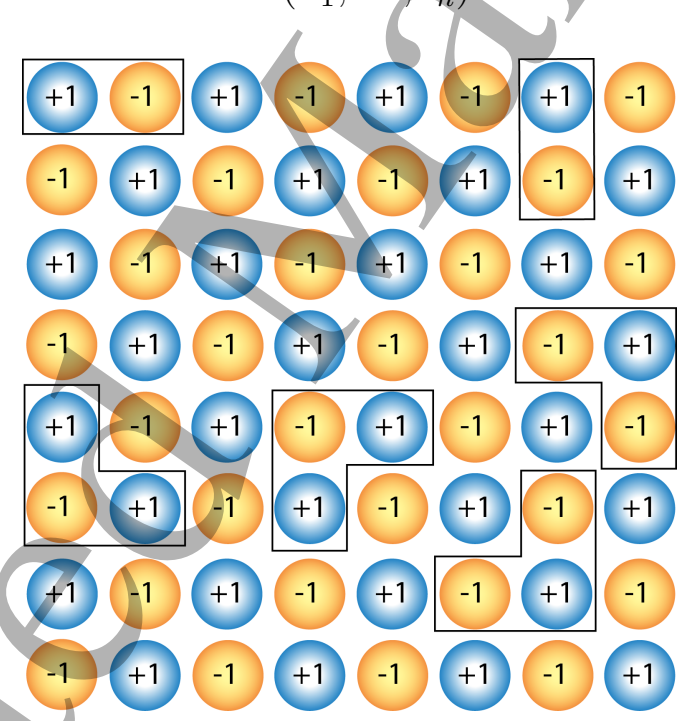

Figure 4: A 2D $(8 \times 8)$ structure including several clusters. +1 and -1 are the lattice sites assigned with different spins.

425 tions) can be grouped into "clusters $(\alpha)$ ". Including all relevant cluster interactions, the 
energy can be expressed as:

$$
E_{\alpha}=\sum_{\alpha} m_{\alpha} J_{\alpha} \bar{\Pi}_{\alpha}(\sigma),
$$

where $m_{\alpha}$ is the multiplicity of the cluster, $\alpha$, and can be obtained by considering all the point symmetries in the lattice cell. $J_{\alpha}$ is the effective cluster interaction (ECI) associated with a cluster, $\alpha . \bar{\Pi}_{\alpha}(\sigma)$ is the correlation matrix of normalised spin-products for a particular cluster of the entire lattice, obtained via:

$$
\bar{\Pi}_{\alpha}(\sigma)=\frac{1}{N m_{\alpha}} \sum_{i \in \alpha} \Pi \sigma_{i}
$$

where $N$ is the number of parent lattice cells required to generate the configuration $\sigma$. Theoretically, the expansion should include all possible clusters. However, that is not practical and one of the key features of cluster expansions is that they usually converge quickly after including a handful of terms. ${ }^{77}$ Consequently, only a relatively small number of DFT calculations are therefore required to parameterise a handful of ECIs. For example, if we calculate the energy of an A-B alloy system and consider only four clusters and four configurations, the energy of each configuration can be expressed as:

$$
\left(\begin{array}{c}
E_{1} \\
E_{2} \\
E_{3} \\
E_{4}
\end{array}\right)=\left(\begin{array}{llll}
\Pi_{1}(1) & \Pi_{2}(1) & \Pi_{3}(1) & \Pi_{4}(1) \\
\Pi_{1}(2) & \Pi_{2}(2) & \Pi_{3}(2) & \Pi_{4}(2) \\
\Pi_{1}(3) & \Pi_{2}(3) & \Pi_{3}(3) & \Pi_{4}(3) \\
\Pi_{1}(4) & \Pi_{2}(4) & \Pi_{3}(4) & \Pi_{4}(4)
\end{array}\right)\left(\begin{array}{c}
J_{1} \\
J_{2} \\
J_{3} \\
J_{4}
\end{array}\right)
$$

55
57
58
9

442 set of clusters with the highest accuracy for predicting configurations achieves the highest 
cross-validation score and is selected.

Various codes exist to link the results of DFT calculations with cluster expansion codes, such as the Alloy Theoretic Automated Toolkit (AT-AT), 180 the Clusters Approach to Statistical Mechanics (CASM), 11 Ab Initio Random Structure Search (AIRSS), ${ }^{82}$ Integrated Cluster Expansion Toolkit (IceT),,$\underline{83}$ and CLuster Expansion in Atomic Simulation Environment (CLEASE).$\underline{84}$ These codes usually provide a means to fit ECIs and include Monte Carlo (MC) features to sample phase spaces. They also allow the generation of DFT calculations to expand the training set. MC methods are explained in the next section.

\subsubsection{Lattice gas and Monte Carlo}

Lattice gas methods simulate the system state as an array of points. ${ }^{85}$ This data structure is ideally suited to represent periodic, crystalline systems, but extensions to more complex systems are possible. In atomistic simulations, the array values denote the occupation of particular sites by certain types of atoms. The evolution of the system state can then be computed in terms of changes in those array values, i.e. site occupancies. $\frac{85}{85}$

In the Ising Hamiltonian described in the previous section, each site can be in either a +1 or -1 state. ${ }^{86}$ This data structure is suited to studying the thermodynamics and kinetics of binary alloys. $\frac{87188}{}$ Simplistically, a $\mathrm{LiB}$ intercalation material can be represented as a binary alloy of lithium atoms and vacancies within an Ising model. $\frac{76[89 \mid 90}{7}$

The interaction Hamiltonian describes how the energy of the system depends on the configuration of the lattice. For a simple interaction model, it is possible to perform a direct evaluation of the partition function, $Z$, via:

$$
Z=\sum_{i} e^{-\beta E_{i}}
$$



be reduced to scale linearly with the number of particles in the system, making the summation computationally tractable. ${ }^{91}$ Measurable quantities, like the open circuit voltage (OCV), voltammograms, and partial molar enthalpy and entropy can be simulated. ${ }^{91-93}$ This approach has been applied to lithium intercalation in lithium manganese oxide (LMO) $\stackrel{93}{\text { and }}$ graphite, ${ }^{9192}$ as demonstrated in section 3.2 .3 . The interactions between the particles can be approximated by taking the average occupation in two levels, allowing ordered structures like graphite stages to be modelled. This approach represents a step in complexity beyond the assumption of simple solid solution behaviour, which is still commonly applied in continuum level models. $\frac{94}{4}$ The approach is closely related to the phase field models applied by Bazant to systems such as lithium iron phosphate (LFP) and graphite. ${ }^{95}$

For a more general and realistic interaction Hamiltonian, the number of energy states precludes direct evaluation of equation 10. In that case, MC methods are useful for calculating thermodynamic properties. This is true for the Ising model defined in section 2.1.4. when represented in more than one dimension, as is the case in most practical systems. It is then more practical to obtain the thermodynamic properties by the Metropolis algorithm. 98 Following the Markov chain of states, the limiting distribution equals the probability distribution of the thermodynamic ensemble. Properties of interest can be obtained from taking the average of sampled configurations once the distribution has reached equilibrium.

Inputting a chemical potential, $\mu$, in the grand canonical ensemble, the ground state properties of the system are obtained as follows. For a LiB, $\mu$ represents the chemical potential of intercalated $\mathrm{Li}$ in the host, i.e. the electrode potential, described in section 2.3.1. Computing the average occupation, $\langle N\rangle$, of particles in the system at each $\mu$ value, therefore allows the equilibrium potential to be simulated at any input temperature, $T$. Along with $\langle N\rangle$, the average internal energy, $\langle E\rangle$, is a useful parameter to check the convergence of the simulation results with respect to the system size. $\frac{85|89| 90 \mid 99}{6}$

Variances can be computed to check the system size convergence and derive experimentally measurable parameters. For example, the configurational component of the heat 


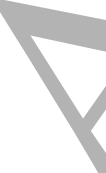

capacity at constant volume, $C_{V}$, given by:

$$
C_{V}=\frac{\beta}{T}\left(\left\langle E^{2}\right\rangle-\langle E\rangle^{2}\right)=\frac{\beta}{T} \operatorname{var}(\mathrm{E})
$$

where $\operatorname{var}(\mathrm{E})$ is the variance of $E$. The vibrational and electronic components of $C_{V}$ must be determined by other means, such as the approaches outlined in section 2.3.4.

It is also possible to determine voltammograms from var $(\mathrm{N})$, as explained by Darling and Newman and Mercer et al. $\frac{89 \mid 99}{}$ If the covariance of $U$ and $N$ is also known, the partial molar internal energy, $\partial U / \partial N$ and partial molar entropy $\partial S / \partial N$ can be obtained, as defined elsewhere. $\frac{89190}{}$ These parameters can be compared with experimental parameters from "entropy

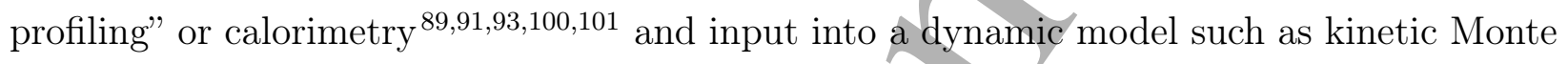
Carlo (kMC), $\frac{76|99| 102 \mid 103}{10}$ or Molecular Dynamics (MD) to describe temperature dependent behaviour. A review of $\mathrm{kMC}$ has recently been published; 104 the technique is also briefly described by Van der Ven et al. ${ }^{9} \mathrm{MD}$ is described in the following section.

\subsubsection{Molecular Dynamics}

$\mathrm{MD}$ is an approach which probes the dynamic evolution of a system over time. The crucial input for these simulations is the potential energy surface (PES), describing the interactions between atoms. In $a b$ initio MD (AIMD), this is described by solving the Schrödinger equation, whereas in a classical (potentials-based) mechanics framework the interactions are described using parameterised interatomic potentials. Here, we give an overview of both frameworks.

AIMD is able to capture/events that potentials-based MD cannot, including bond breaking, and bond formation. AIMD also assumes that the dynamics of particles can be treated classically and that the equation of motion for all particles can be written as:

$$
M_{I} \ddot{\mathbf{R}}_{I}=-\nabla_{I}\left[\varepsilon_{0}(\mathbf{R})+V_{N N}(\mathbf{R})\right]
$$


where $M_{I}$ is the mass of a given nucleus, $\mathbf{R}$ denotes all nuclear coordinates, $\nabla_{I}$ is the Laplacian operator of a given nucleus, $\varepsilon_{0}(\mathbf{R})$ represents the ground state energy of the system at that given nuclear configuration, and $V_{N N}(\mathbf{R})$ represents the nuclear-nuclear coulomb repulsion at that given nuclear configuration.

Most modern techniques use KS-DFT (c.f. section 2.1.1) to solve the Schrödinger equation which finds the ground state energy. AIMD can be broadly split up into two main categories: Born-Oppenheimer dynamics and Car-Parrinello extended Lagrangean. The Born-Oppenheimer dynamics method uses a symplectic integrator to numerically integrate the equation of motion in Eq. 12 for each time step. The Car-Perrinello extended Lagrangean method gives the Kohn-Sham orbitals an artificial time-dependence. To attain a minimum energy with each new $\mathbf{R}$, the orbital dynamics are kept at a temperature much lower than that of the nuclei, but still high enough for the orbitals to quickly relax as the equation of motion proceeds. The new orbitals and their dynamics can then be defined by the Lagrangean equation: $\frac{105}{105}$

$$
L=\mu \sum_{i} f_{i} \int d \mathbf{r}\left|\psi_{i}(\mathbf{r}, t)\right|^{2}+\frac{1}{2} \sum_{I=1}^{N} M_{I} \dot{\mathbf{R}}_{I}^{2}(t)-E[\psi(t), \mathbf{R}(t)]+\sum_{i, j} \Lambda_{i j}\left[\int d \mathbf{r} \psi_{i}^{*}(\mathbf{r}, t) \psi_{j}(\mathbf{r}, t)-\delta_{i j}\right]
$$

where $\mu$ is an artificial kinetic energy term (discussed further in Refs. 106 and 107), $\psi_{x}(\mathbf{r}, t)$ are the time-dependent Kohn-Sham orbitals, and $\Lambda_{i j}$ contains a set of Lagrange multipliers to implement the orthonormality constraint on the orbitals.

Potentials-based MD is not able to capture some of the finer details of the system dynamics that AMD is able to, however, it is able to reach longer time- and length- scales, providing information on long range diffusion properties. In classical potentials-based MD, the atomic interactions are described using parameterised interatomic potentials. There are multiple forms interatomic potentials can take, with their relevancy and accuracy relating to the system and study being conducted. Atoms are either attracted or repelled by one an- 
other based on their interatomic distance, $r$, to reduce their potential energy to a minimum, $r_{e q}$. This is known as a pair-interaction, which can be used to calculate the force, $\overrightarrow{\mathrm{F}}$, acting on each atom, given by:

$$
\overrightarrow{\mathrm{F}}_{\mathrm{i}}=\sum_{j} \vec{\nabla} E\left(r_{i j}\right)
$$

In complex systems, there is a "net effect" of the $N$ surrounding atoms which can be accounted for by calculating the vector summation of each pair interaction contribution. Within ionic materials, the pair interactions are dominant and therefore it is computationally tractable to truncate the expression after the first term ${ }^{108}$ to give an approximation of the pair potential. The charged nature of ions forms a coulombic interaction, where the relatively slow decay of $\frac{1}{r}$ as $r$ increases, gives rise to the long range component of the potential. The general term for the total potential can therefore be written as:

$$
E\left(r_{i j}\right)=\frac{Q_{i} Q_{j}}{4 \pi \varepsilon_{0} r_{i j}}+\Phi_{s r}
$$

where $i$ and $j$ are ions of charge $Q_{i}$ and $Q_{j}$ at a distance of $r_{i j}$, and $\varepsilon_{0}$ is the permittivity of free space. $\Phi_{s r}$ is used to denote the remaining short-range interactions.

For ionic solids, including cathode materials, a common choice for an interatomic potential is a Coulomb-Buckingham potential, $\underline{109}$ derived from the Born model of the ionic solid, $110 \mid 111$ where the potential energy of the system can be expressed as:

$$
E\left(r_{i j}\right)=\sum_{i j} \frac{Q_{i} Q_{j}}{4 \pi \varepsilon_{0} r_{i j}}+\sum_{i j} A \exp \left(\frac{-r_{i j}}{\rho}\right)-C r_{i j}^{-6},
$$

where, $A, \rho$, and $C$ are constants.

MD simulations can be performed using a range of ensembles, with the most commonly used being microcanonical (NVE), canonical (NVT), and isothermal-isobaric (NPT) ensembles.112 114 Here, the number of atoms $(\mathrm{N})$, volume (V), energy (E), temperature (T), and ${ }_{557}$ pressure $(\mathrm{P})$ are conserved within the respective ensembles. Within the NVT and NPT 
ensembles the energy of endothermic and exothermic processes is exchanged with a thermostat. A variety of thermostat algorithms are available, with some of the most popular methods including the Nosé-Hoover, Berendsen, and Andersen thermostats. ${ }^{112}[114$ For NPT ensembles, a barostat is also applied to control pressure.

The choice between AIMD and potentials-based MD is a trade-off between computational cost, accuracy, and transferability. AIMD is highly accurate, however, it is computationally expensive and scales poorly $\left(>O\left(N^{3}\right)\right)$, making reachable system sizes and timescales relatively small (<1000 atoms, $\sim 100 \mathrm{ps}$ ). On the other hand, potentials-based MD is less computationally expensive and can be applied to much larger system sizes, up to millions of atoms, with longer reachable time scales in the range of nanoseconds. However, the potentials-based approach is generally less accurate, as developing an interatomic potential which is sufficiently accurate enough to describe the specific system chemistry is challenging. The development of interatomic potentials is discussed in greater detail in section 2.2.2. More recently, development of linear-scaling DFT approaches, as discussed in section 2.1.2, has worked towards reducing this trade-off.

\subsection{Method Development}

\subsubsection{Continuum models of electrolyte solutions within Density Functional The-} ory

Electrode-electrolyte interfaces are an important part of LiBs and an area of active research. $\frac{115 \mid 116}{116}$ The complexity of the structure and formation of electrical double layers at the interface has hindered the understanding of important electrochemical processes. While DFT-based electronic structure methods have been successfully used to study the solid-state physics in the bulk electrodes of LiBs, they are inadequate to describe the liquid state, which lacks structural order. This has led to rapid development of methods to describe the electrode-electrolyte interfaces. 117

The liquid state can be described mainly via explicit solvation, $\frac{118}{1}$ implicit solvation,, 119 


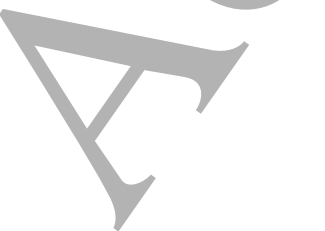
models. $66 \mid 128] 135$ $c_{i}(\mathbf{r})$, as: 135 the P-BE:

or both. $\frac{120}{120}$ the former, the surrounding solvent and electrolyte molecules are considered at the same level of chemical accuracy as the electrode atoms. The surrounding solvent and electrolyte molecules can not only neutralise the excess charge on the electrode surface, but also form bonds and adsorb on the electrode surface. $121-123$ The addition of a large number of solvent and electrolyte molecules to describe the liquid state drastically increases the configurational degrees of freedom. Sampling this large configurational space is computationally demanding and often leads to loss of focus on the main region of interest: the interface. While consideration of the first bonding layer of explicit solvent and electrolyte molecules is necessary to describe the local effects of bonding and electric field, 124 the degrees of freedom of the non-participating solvent and electrolyte molecules far away can be averaged out via an implicit model of the electrolyte solution. 125126 The electrostatic potential in these hybrid quantum-continuum models is obtained from the solution of the Poisson-Boltzmann equation (P-BE). $\stackrel{127}{ }$ Recently, many DFT codes have integrated P-BE based continuum

The continuum electrolyte ions with space-dependent concentrations, $c_{i}(\mathbf{r}), i=1 \ldots p$, and charges, $\left\{z_{i}\right\}$, create a mobile electrolyte density, $\rho_{\text {mob }}(\mathbf{r})=\sum_{i=1}^{p} z_{i} c_{i}(\mathbf{r})$, which interacts with the quantum charge density, $\rho(\mathbf{r})$, within a mean-field electrostatic potential, $\nu(\mathbf{r})$. This effect can be included in standard DFT by extending the standard free energy functional to include the mean-field electrostatic potential, $\nu(\mathbf{r})$, and the mobile charge concentrations,

$$
E[\rho(\mathbf{r})] \rightarrow \Omega\left[\rho(\mathbf{r}), \nu(\mathbf{r}), c_{i}(\mathbf{r})\right]
$$

The variation of the free energy functional with the electrostatic potential, $\nu(\mathbf{r})$, gives

$$
\nabla \cdot[\varepsilon(\mathbf{r}) \nabla \nu(\mathbf{r})]=-4 \pi\left[\rho(\mathbf{r})+\rho_{\mathrm{mob}}(\mathbf{r})\right]
$$


The P-BE not only includes the quantum charge density, $\rho(\mathbf{r})$, as in standard DFT calculations in vacuum, but also the effect of the solvent in terms of a continuum dielectric with permittivity function, $\varepsilon(\mathbf{r})$, and mobile charge density of electrolyteions, $\rho_{\text {mob }}(\mathbf{r})$. The permittivity function is chosen as a smooth function with value varying from 1 in the quantum region to $\varepsilon^{\infty}$ in the solvent region: 131

$$
\varepsilon(\mathbf{r})=1+\left(\varepsilon^{\infty}-1\right) s(\mathbf{r})
$$

where $s(\mathbf{r})$ is a smooth interface function varying from 0 in the quantum region to 1 in the solvent. Several choices for the interface function have been discussed by Andreussi et al. ${ }^{136}$ The variation of the free energy functional with ion concentrations, $c_{i}(\mathbf{r})$, gives the Boltzmann expression for ionic concentrations:

$$
c_{i}(\mathbf{r})=c_{i}^{\infty} \lambda(\mathbf{r}) \exp \left(-\frac{z_{i} \nu(\mathbf{r})}{k_{\mathrm{B}} T}+\frac{\mu_{i}^{\mathrm{ex}}}{k_{\mathrm{B}} T}\right), i=1 \ldots p,
$$

where $\left\{c_{i}^{\infty}\right\}$ and $\left\{\mu_{i}^{\text {ex }}\right\}$ are the bulk concentrations and excess chemical potentials of the electrolyte ions. The mobile charge density of electrolyte ions, $\rho_{\mathrm{mob}}(\mathbf{r})=\sum_{i=1}^{p} z_{i} c_{i}(\mathbf{r})$, is shown schematically in Fig. 5. As the interaction with mobile electrolyte charge is purely electrostatic and excludes any quantum effects such as Pauli repulsion, there is a problem of electrolyte charge accumulating infinitely close to the electrode. In order to prevent this problem, the models include an electrolyte accessibility function, $\lambda(\mathbf{r})$, which varies from 0 near the electrode to 1 in the bulk electrolyte region. $\frac{133 \mid 137 / 138}{13}$ One of the ways of defining such an accessibility function is as a product of atom-centred interlocking spheres of error functions: 135

$$
\lambda(\mathbf{r})=\prod_{k}^{n_{\text {atoms }}} \frac{1}{2}\left[1+\operatorname{erf}\left(\frac{\left|\mathbf{r}-\mathbf{R}_{k}\right|-R_{k}^{\text {solute }}\left(\rho_{\mathrm{e}}^{\lambda}\right)-R_{k}^{\text {solvent }}}{\sigma}\right)\right],
$$

where $\sigma$ is a smearing width $\left(0<\sigma<0.5 a_{0}\right)$. This description of the ion exclusion region derives from a physical picture: the electrolyte ions are moved away from the quantum 


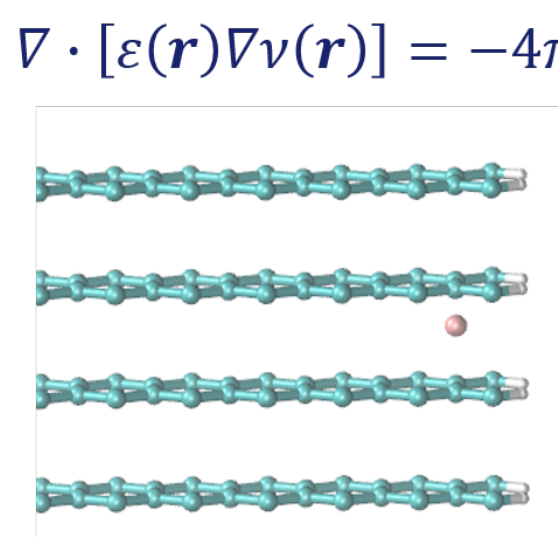

Figure 5: DFT simulation of a lithiated graphite interface in contact with an implicit electrolyte solution, based on the solution of the Poisson-Boltzmann equation. Reprinted with permission from Ref. 135. Copyright 2020 American Chemical Society.

electrode, up to a distance that incorporates not only the size of the species but also a solvation shell radius around the electrolyte ions. The species size can be described in terms of an isoradius of electronic density, $\rho_{\mathrm{e}}^{\lambda}$. The solvation shell radius, $R_{k}^{\text {solvent }}$, depends on the solvent and is added to the species size, to calculate the overall radius of interlocking spheres for the accessibility function.

The electrostatic potential, $\nu(\mathbf{r})$, obtained from equation 18 is due to the entire electrodeelectrolyte interface, where the electrode, is treated quantum mechanically and the electrolyte solution as a continuum. Variation of the free energy functional with electronic density gives the Kohn-Sham equations in the total electrostatic potential, with additional terms for the variation of interface function with electronic density. ${ }^{[301139]}$ Solvation energies are defined as: 1301133
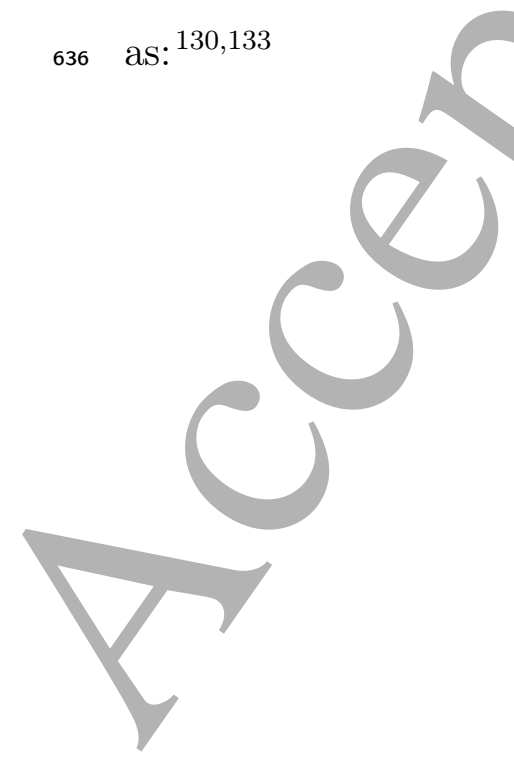

$$
\begin{aligned}
\Delta \Omega & =\Omega-\Omega_{\mathrm{vac}}-\Omega_{\text {electrolyte }} \\
& =\Omega\left[\rho(\mathbf{r}),\left\{c_{i}(\mathbf{r})\right\}, \nu(\mathbf{r})\right] \\
& -\Omega\left[\rho_{\mathrm{vac}}(\mathbf{r}),\left\{c_{i}(\mathbf{r})\right\}=0, \nu_{\mathrm{vac}}(\mathbf{r})\right] \\
& -\Omega\left[\rho(\mathbf{r})=0,\left\{c_{i}(\mathbf{r})\right\}=\left\{c_{i}^{\infty}\right\}, \nu(\mathbf{r})=0\right],
\end{aligned}
$$


where the respective terms can be computed as the total free energy in the electrolyte solution, the total free energy in vacuum, and the total free energy of the pure electrolyte. $\frac{135}{1}$ The electrolyte effect on solvation energies can be computed as the difference of solvation energy in electrolyte at $\left\{c_{i}^{\infty}\right\}$ and solvation energy in pure solvent at $\left\{c_{i}^{\infty}=0\right\}$ :

$$
\begin{aligned}
\Delta \Delta \Omega & =\Delta \Omega\left[\left\{c_{i}^{\infty}\right\}\right]-\Delta \Omega\left[\left\{c_{i}^{\infty}=0\right\}\right] \\
& =\Omega-\Omega_{\text {sol }}-\Omega_{\text {electrolyte }}
\end{aligned}
$$

where the respective terms are computed as the total free energy in the electrolyte solution, $\left\{c_{i}^{\infty}\right\}$, the total free energy in pure solvent, $\left\{c_{i}^{\infty}=0\right\}$, and the total free energy of the pure electrolyte.

\subsubsection{Fitting Potentials for Classical Molecular Dynamics}

The development of sufficiently accurate interatomic potentials for a specific chemistry is quite challenging. Interatomic potentials are traditionally based on mathematical functions that have been parameterised using experimental and/or electronic structure methods derived data. $\frac{109 \mid 140}{1}$ There are a limited number of codes available with the explicit purpose or functionality for fitting potentials. Here, we present several available codes and discuss the complexities and considerations involved in deriving accurate interatomic potentials.

GULP, 141 the General Utility Lattice Program, is a widely used code for performing a variety of simulation types on materials using boundary conditions. $\frac{114}{11}$ Within this code, there is the functionality to fit interatomic potentials to either experimental measurements or electronic structure methods data. ${ }^{142}$ GULP is capable of simultaneous fitting to multiple structures and can also handle core-shell models (which capture polarisation of atoms).

Atomicrex, $, \frac{143}{d f t f i t}, \frac{144}{14}$ and potfit ${ }^{145 \mid 146}$ are codes designed to fit potentials to electronic structure methods data. Each of these codes have different levels of flexibility and 
their own unique features, however, a joint limitation is the ability to fit empirical potentials is limited to rigid ions and cannot fit a core-shell model.

During the process of developing potentials for $\mathrm{Li}\left(\mathrm{Ni}_{x} \mathrm{Mn}_{y} \mathrm{Co}_{z}\right) \mathrm{O}_{2}(\mathrm{NMC})$, and its ternary system $\mathrm{LiNiO}_{2}$, it was found that none of these codes are able to accurately produce potentials for these materials. The complex nature of Ni chemistry in a layered oxide material is challenging, and to the best of our knowledge, no interatomic potentials exist for $\mathrm{Ni}^{3+}$. Oxide systems are widely described using a Buckingham potential form, as given in equation 16, and for layered structures, including NMC and its ternary systems, variations of the Buckingham potentials are presented. Some use rigid ion/models, $\frac{147+150}{150}$ others use core-shell models, $147 \mid 151-156$ and a mixture of formal and partial charges have been implemented. With literature in disagreement over which variation of the Buckingham potential is the most accurate for representing the system, a code capable of fitting different permutations of the Buckingham potential is needed.

Structure and composition of a material are crucial to determine the functional form of the potential. For example, for a layered structure such as NMC-811, it is crucial to consider polarisability. Polarisability is described in classical (potentials-based) MD using a core-shell model. There are predominately two types of core-shell models: the relaxed (massless shells) model ${ }^{157}$ and the dynamic (adiabatic shells) model. 158 The adiabatic shell model is more widely used in literature, including all core-shell related cited works in this section,,$\underline{147151-156}$ for calculating long trajectories, as it is less computationally taxing. In the adiabatic shell model, a fraction of the atomic mass is assigned to the shell. There is no defined fraction size; however, placing $10 \%$ of the atomic mass on the shell is considered common practice. ${ }^{112113}$ An additional consideration for using a core-shell model is the separation of the formal atomic charge across the core and shell. However, determined numerical values of the core-shell charge separation are inconsistent. $156 \mid 159$ 162 In some systems, where there is high polarisability, such as in LMO, the short-range interactions are overwhelmed by the longer-range coulombic term. In these cases, the system charges can be scaled to 
increase the influence of the short-range interactions, and are termed partial charges. The scaling factor is system dependent therefore no specific value is ideal in all cases, however 60 $\%$ formal charge is commonly adopted. 163

POtential Parameter Optimisation for Force-Fields (PopOff), 164 a code developed within the Faraday institution, was specifically created for fitting different permutations of the Buckingham potential. It is unique in its ability to consider all the factors discussed above (rigid ion/core-shell/charge separation/charge scaling) in a modular design, allowing flexible fitting to suit individual systems. The code has been developed in Python, using a training set consisting of DFT derived data $(D F T)$ and utilising the Large-scale Atomic/Molecular Massively Parallel Simulator (LAMMPS). ${ }^{113}$ The potential is fitted by minimising the mean squared error $\left(\chi^{2}\right)$ between the DFT forces, $F^{D F T}$, and stress tensors, $\sigma^{D F T}$, and those produced using the fitted interatomic potential $\left(F^{I P}, \sigma^{I P}\right)$, defined as:

$$
\chi^{2}=\sum_{i, \alpha}^{N} \frac{\left(F_{i, \alpha}^{D F T}-F_{i, \alpha}^{I P}\right)^{2}}{N_{i}}+\sum_{\beta} \frac{\left(\sigma_{\beta}^{D F T}-\sigma_{\beta}^{I P}\right)^{2}}{6}
$$

This modular design allows the construction of a Buckingham potential able to accommodate the considerations and complexities of different systems. PopOff also allows for individual parameters to be fixed/excluded from the fit, lowering the fit dimensionality and computational cost. This is particularly useful for excluding dispersion terms, which are known to be zero or close to, for a range of elements. $161 \mid 165$

\subsection{Calculating observable properties}

\subsubsection{Equilibrium voltage}

The equilibrium cell voltage, $E(x)$, where $0<x<1$ denotes the fraction of sites occupied by lithium in the intercalation host, is a fundamental thermodynamic quantity related to the energy density of a cell. $\frac{9166 \mid 167}{} E(x)$ can be probed through experimental measurements of the open circuit voltage $(\mathrm{OCV})$, that is, the voltage between the cathode and anode 
terminals under zero current flow, assuming that the system has been given sufficient time for the OCV to relax to the value of $E(x)$. Computationally, the equilibrium cell voltage can be modelled through DFT calculations at $T=0 \mathrm{~K} ; 911661167$ the effect of thermal fluctuations can be included by modelling using Monte Carlo (MC) calculations.

There is a fundamental relationship between the Gibbs free energy of lithium dissolution into the host, $G(x)$, the chemical potential of Li intercalation in the host, $\mu(x)$, and the cell voltage $E(x)$. Knowledge of $G(x)$ also provides information about the evolution of the phase behaviour dependent on the fraction of intercalated Li,, 1761167168 enabling the construction of phase diagrams from DFT. The relationships are represented schematically in Figure 6. In essence: the tangent to the free energy curve, $G(x)$, allows $\mu(x)$ and hence the cell voltage to be obtained. Alternatively, integration of $\mu(x)$ can be used to derive free energy curves.

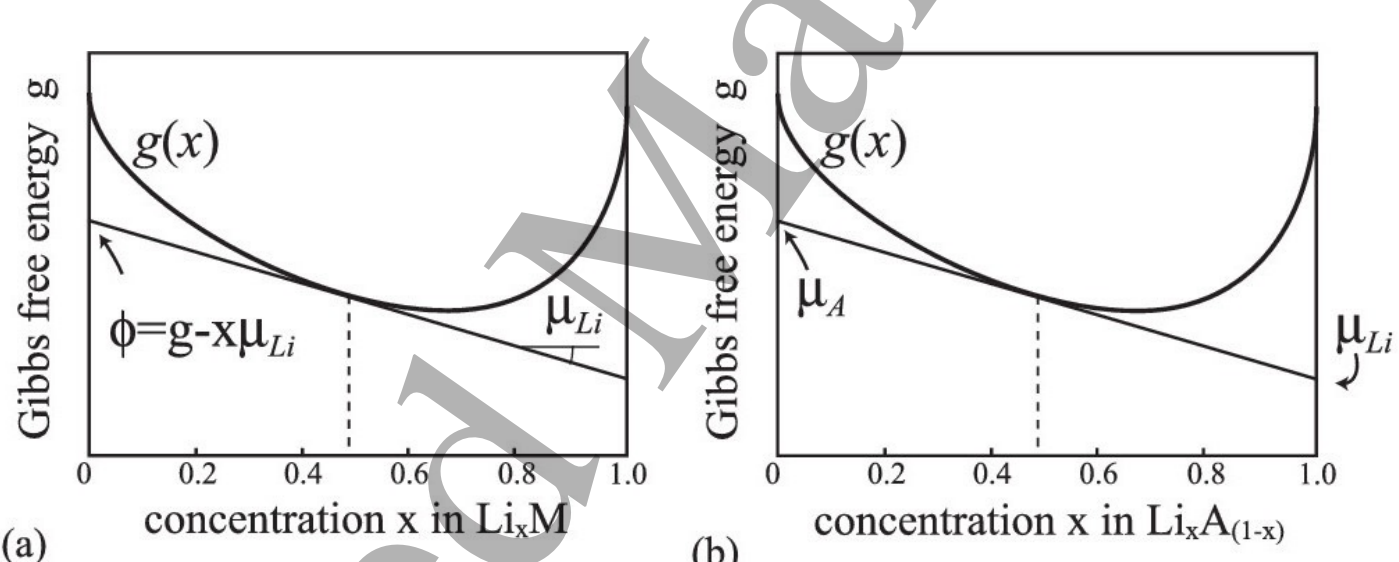

Figure 6: Representation of the connection between the Gibbs free energy, $G(x)$, the lithium chemical potential $\mu(x)$ in (a) an intercalation electrode and (b) an alloy electrode. Reprinted with permission from Ref. 9. Copyright 2020 American Chemical Society.

In the case of a Li-ion cell, the equilibrium cell voltage, $\phi(x)$, and the chemical potential of intercalated Li, $\mu(x)$, are related by:

$$
\phi(x)=-\frac{\mu(x)-\mu_{\mathrm{Li}}^{\mathrm{ref}}}{n F},
$$

721 where $\mu_{\mathrm{Li}}^{\mathrm{ref}}$ is the chemical potential of the reference electrode, $n$ is the number of electrons 722 transferred per formula unit of intercalation host ( $n=1$ for Li-ion cells), and $F$ is the Faraday 
737

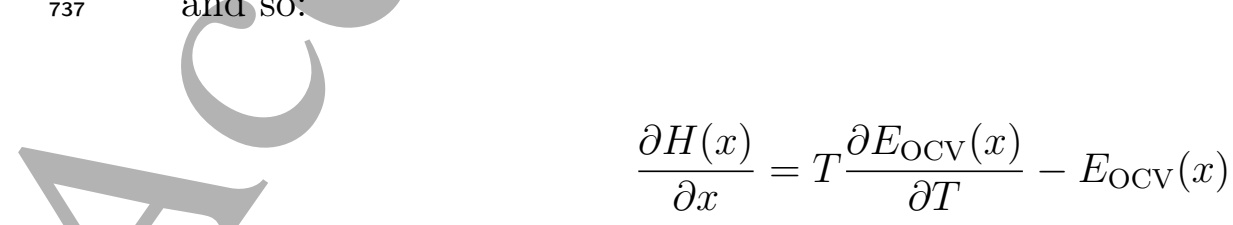
assumed to be constant from now on and dropped, to simplify notation.

Similarly, it is well known that:

$$
\frac{\partial G(x)}{\partial x}=\frac{\partial H(x)}{\partial x}-T \frac{\partial S(x)}{\partial x}
$$
material. of the OCV with/respect to $T$ and using the chain rule, we obtain:

$$
\frac{\partial S(x)}{\partial x}=\frac{\partial E_{\mathrm{OCV}}(x)}{\partial T}
$$

where $\underline{G}$ is the absolute (i.e. extensive) Gibbs free energy of Li dissolution into the host, $p$ is pressure, $T$ is the absolute temperature, and $N_{\text {host }}$ and $N_{\mathrm{Li}}$ are the number of host and lithium atoms in the system, respectively. The subscripts $p, T$, and $N_{\text {host }}$ will be implicitly

where $H(x)$ and $S(x)$ are the enthalpy and entropy, respectively, per formula unit of host

We can use equations 28, 29, and 30 to get $\partial G / \partial x=-E_{\mathrm{OCV}}$, then, taking the derivative 
Due to the units of electron Volts $(\mathrm{eV})$ per formula unit for the potentials $H(x)$ and $T S(x)$, i.e. as in the conversion between equations 27 and 28 , the usual factors of $F$ have been omitted. In this way it is possible to simulate not only the equilibrium voltage, but split its contributions into enthalpy and entropy components. Both components can be experimentally measured $\frac{01 / 93 / 100 / 169 \mid 170}{1}$ and modelled through MC or mean field methods, $\underline{89 \mid 91,93}$ providing additional properties for model validation purposes and to check the temperature dependence of those properties is modelled accurately. A good thermodynamic basis can then be used to derive dynamic properties, as outlined in the subsequent sections.

\subsubsection{Activity coefficients of electrolytes}

The activity coefficients of electrolytes $\left(\gamma_{j}, j=1 \ldots p\right)$ describe the thermodynamics of nonideal solutions. 171 The activity coefficient of electrolytes can be computed from DFT $+\mathrm{P}-\mathrm{BE}$ models, as described in section 2.2.1, by computing the electrolyte effect on solvation energies, $\Delta \Delta \Omega: \underline{130 \mid 135}$

$$
\ln \gamma_{j}=\frac{\Delta \Delta \Omega_{j}\left[\left\{c_{i}^{\infty}\right\}\right]}{k_{\mathrm{B}} T}, j=1 \ldots p
$$

For an electrolyte dissociating into $p$ species, the mean activity coefficient can be calculated as:

$$
\ln \gamma_{\text {mean }}=\frac{1}{p} \sum_{j=1}^{p} \ln \gamma_{j}
$$

\subsubsection{Diffusion coefficients}

The diffusion coefficient is a term used to describe the rate of ion transport within a system. This term, however, has been used in literature to express several forms of diffusion, which characterise diffusion in a material in different ways. Here, we describe several commonly used forms of diffusion coefficient, in context of where they are used, focusing on bulk diffusion. Heitjans and Kärger gives a detailed description of diffusion along grain boundaries 
and along surfaces (chapters 7 and 8). 172

Ionic transport within the electrodes and electrolyte plays a vital role in the kinetics of a battery. It can be described fundamentally with flux expressions that relate ion fluxes to chemical or electrochemical potential gradients. This is related by Fick's first law, where the diffusion flux, $\boldsymbol{\jmath}$, is described using the gradient of the concentration, $c$, via:

$$
\boldsymbol{\jmath}=-\mathcal{D} \nabla c
$$

where $\mathcal{D}$ is denoted as the diffusion coefficient tensor or diffusivity tensor and implies that $\mathcal{D}$ varies with direction. In general, the diffusion flux and concentration gradient are not always antiparallel. They are antiparallel for isotropic médiums. Heitjans and Kärger discusses this in more detail. 172

Steady state methods for measuring diffusion coefficients, like the permeation method, are directly based on Fick's first law. ${ }^{173}$ In non-steady states, the diffusion flux and concentration vary with time, $t$, and position $x$, and a balanced equation is necessary. For particles which undergo no reaction this become the continuity equation:

$$
\frac{\partial c}{\partial t}+\nabla \boldsymbol{\jmath}=0
$$

Combining equations 35 and 36 leads to Fick's second law, also called the diffusion equation, which predicts how diffusion causes the concentration to change with time:

$$
\frac{\partial c}{\partial t}=\nabla(\mathcal{D} \nabla c)
$$

In diffusion studies with trace elements the material composition does not practically change and $\mathcal{D}$ is independent of the tracer concentration, presenting a concentration-independent diffusion coefficient. For diffusion in multiple dimensions Fick's second law becomes: $\frac{174}{17}$

$$
\frac{\partial c}{\partial t}=\mathcal{D} \nabla^{2} c
$$




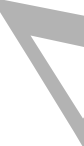

The temperature dependence of the diffusion coefficient is often described empirically by an Arrhenius relation:

$$
\mathcal{D}=\mathcal{D}_{0} \cdot \exp \left(-\frac{E_{A}}{k_{B} T}\right)
$$

where $E_{A}$ is the activation energy for the mass transport, $D_{0}^{T}$ is the pre-exponential factor, $k_{B}$ is the Boltzmann constant, and $T$ is the temperature.

From the microscopic point of view, the tracer diffusion coefficient can be defined by the Einstein-Smoluchowski relation: $\underline{175 \mid 176}$

$$
\mathcal{D}=\lim _{t \rightarrow \infty} \frac{\left\langle r^{2}(t)\right\rangle}{2 d t}, \text { where }\left\langle r^{2}(t)\right\rangle=\left\langle\left(x(t)-x_{0}\right)^{2}\right\rangle,
$$

where, $\left\langle r^{2}(t)\right\rangle$ is the mean square displacement (MSD) of the particles after time $t$ and $d$ is the dimensionality of the movement. This is also known as the self diffusion coefficient and is the main approach used to calculate the diffusion coefficient in $\mathrm{kMC}$ and MD from the atom trajectories. Van der Ven et al. discusses in greater detail. 9

In atomistic modelling, diffusion coefficients can also be calculated using other approaches, such as Green-Kubo. The Green-Kubo approach is linked to the Einstein-Smoluchowski relation approach, equation 40, Both approaches assume that particle dynamics can be well approximated by Brownian motion. As described in equation 40, Brownian motion of independent particles can be expressed by the MSD of a particle proportional to time. This can also be termed as the integral of the velocity. The Green-Kubo approach is derived from the integration of the velocity (or current) autocorrelation function. Assuming that dynamics is ergodic, the diffusion coefficient can be calculated using a linear fit to the velocity autocorrelation function. Averaging is applied to this, for example, a time average for a selected particle type, a sample average, or an ensemble average. 
797

\subsubsection{Vibrational and Thermal Properties}

While MD simulates the evolution of a chemical system over time, lattice dynamics is an approach that models the underlying vibrations. In crystalline solids, extended vibrations can be described as phonons with a characteristic frequency and wavevector, $\omega(q)$. A unit cell with $N$ atoms contains $3 N$ phonon modes. The theory of phonons provides a direct connection between microscopic atomic motion and macroscopic properties including specific heat capacity, IR and Raman spectra, and thermal expansion. $177+179$

While assuming that phonons are harmonic simplifies the theoretical description, it is necessary to include anharmonic effects to describe phenomena such as heat transport. The lattice thermal conductivity, $\kappa$, depends on the lifetime of each phonon, i.e. how long it persists before decaying, which is an anharmonic process. Formally, the thermal conductivity given by the product of the modal heat capacity, $\left(C_{V}\right)$, the group velocity, $v$, and the phonon mean free path, $v \times \tau$ (where $\tau$ is the phonon lifetime). The macroscopic $\kappa$ is obtained by summing over band indices, $v$, averaging over wavevectors, $q$, and normalising by the unit cell volume:

$$
\kappa=\frac{1}{N V_{0}} \sum_{q v} C_{V, q v} v_{q v} \otimes v_{q v} \tau_{q v}
$$

where $N$ is the number of unit cells in the crystal (number of wavevectors in the Brillouin zone summation) and $V_{0}$ is the volume of the crystallographic unit cell.

The heat capacity and group velocity can be extracted from the harmonic phonons, which are readily accessible from calculations based on electronic structure methods or potentialsbased potential methods. The lifetime of each phonon mode is more demanding to compute and is often performed within a many-body perturbation theory expansion of phonon-phonon interactions. One approximation is to consider only the leading term of three-phonon creation and annihilation. $\frac{180}{10}$ However, higher-order processes may limit the lifetimes, depending on the material and temperature. There are a range of packages available to compute the 
terms in equation 41 including PHONO3PY ${ }^{180}$ (recently applied to $\mathrm{LiCoO}_{2}$ and $\mathrm{NMC}^{-1}$ cathodes,, 181182 ALAMODE, 183 and SHENGBTE. 184

\section{Anodes}

\subsection{Introduction}

Critical to the success of lithium-ion batteries (LiBs) was the development of graphite-based anodes. Graphite proved to be ideal for this application, due to its low (de)-intercalation potential, only slightly higher than that of metallic lithium, and high theoretical gravimetric capacity of $372 \mathrm{mAh} \mathrm{g}^{-1}$. However, many key degradation mechanisms in present-day LiBs that lead to their eventual failure, including cracking/reformation of the solid-electrolyte interphase (SEI) and lithium plating, are still intimately connected with graphite-based anodes. $\frac{185[186}{1}$ The understanding of these mechanisms is still far from complete and leads to complex, non-linear degradation behaviour that is difficult to predict, $\stackrel{187}{ }$ motivating the development of multiscale models with a descriptive and predictive capability. A critical starting point for these models is a physically accurate atomistic description of the graphite and its interface with organic electrolytes.

The possibility to form Li-graphite intercalation compounds (Li-GICs), also known as "stages", up to a stoichiometry of $\mathrm{LiC}_{6}$, was known in 1975 , albeit at that time it was only possible to form them by heat treating powders. 188 -190 Initial attempts to electrochemically intercalate lithium into graphite resulted in co-intercalation of the organic solvent and exfoliation of the graphite. 191 In 1983, Yazami and Touzain reported the first successful intercalation into graphite using a solid polymer electrolyte. 192 Fong et al. found that reversible lithium intercalation could be achieved in liquid organic electrolytes using ethylene carbonate (EC) as part of the solvent, which finally enabled the formation of a stable SEI on the graphite surface. ${ }^{193}$ Mixtures of EC and dimethyl carbonate (DMC) were developed by Tarascon and Guyomard in $1993^{194}$ and present-day graphite-based LiBs are still primarily 
based on this electrolyte mixture. The key challenge was finding a solvent chemistry that provided sufficient ionic conductivity, did not decompose significantly at the $\sim 4 \mathrm{~V}$ cathode potential, while also avoiding co-intercalation into the graphite and producing a stable SEI on its surface. Further incremental improvements in performance have since been achieved through additional additives and, more recently, the inclusion of small amounts of silicon in the anode as a secondary material.

This section predominantly focuses on graphite, since it remains the primary anode electrode material in the majority of commercial lithium ion (Li-ion) cells. $\frac{14}{14}$ Here, the experimentally confirmed Li-graphite stages and the nomenclature necessary for atomistic models of bulk behaviour are defined. Atomistic modelling in the graphite bulk is outlined, including both thermodynamic and kinetic properties. The key graphite surfaces relevant to understanding the initial intercalation are described, then moving to modelling at the graphite edges and the interface with the electrolyte. Throughout, it is shown how these models enable quantitative understanding of the physical mechanisms of Li intercalation in the graphite bulk, the initial insertion at the graphite edges, and the interface between graphite and the electrolyte. Along the way, the key experimentally observable parameters are outlined, showing success stories of atomistic models to not only quantify and describe those parameters but to also predict new behaviour. In some cases, quantitative disagreement between model and experimental observations is also informative and can create new research directions. Work linking atomistic and continuum models is presented in the case of the technologically important SEI. Given the emerging importance of $\mathrm{C} / \mathrm{Si}$ and $\mathrm{C} / \mathrm{SiO}_{x}$ composites in commercial anode materials, some of the challenges in atomistic modelling of Si and related materials are summarised at the end. In the outlook, key remaining challenges are presented for modelling not only graphite, but also next generation materials such as silicides. Challenges related to metallic Li formation on graphite anodes, and the use of metallic Li as an anode material, are also summarised in the outlook. 
872

873

874

875

876

877

878

879

880

881

882

883

884

885

\subsection{Bulk Properties}

\subsubsection{Graphite structure and Li-graphite stages}

Graphite possesses a layered structure with carbon atoms forming a network of hexagons/in each layer. The carbon atoms located within one layer are covalently bonded to each other, whereas the weak interlayer binding arises from the dispersion or van der Waals (vdW) interactions. (Figure 7b), but synthesised graphite structures also contain a small amount of rhombohedral (ABC-stacked) domains. 198

Li-graphite stages, also known as lithium-graphite intercalation compounds (Li-GICs), are lithium concentration-dependent structures of various stoichiometries. ${ }^{[88|189| 195|197 / 199|}$ In Li-GICs, Li atoms form a $2 \mathrm{D}$ hexagonal $(\sqrt{3} \times \sqrt{3}) \mathrm{R} 30^{\circ}$ superstructure, with Li atoms sitting directly above each other, as shown in Figure $7 \mathrm{a}$. The stage number, $n$, denotes the number of graphene layers between each lithium-filled layer. $\frac{188 / 195 / 197 / 200}{1}$ The experimentally confirmed stages adopt different stackings in the carbon host lattice, as shown in Figure 7. The standard nomenclature for GICs $\frac{189}{10}$ denotes the carbon stacking and Li occupancies: periodic carbon layer stackings along the [001] axis are designated by uppercase letters separated by Greek lowercase letters, if Li is intercalated between planes. For instance, fully lithiated Stage $\mathrm{I} \mathrm{LiC}_{6}(x=1)$ adopts $\mathrm{A} \alpha \mathrm{A} \alpha \mathrm{A} \alpha$ stacking. $170 \mid 197 / 201$ Here $\alpha$ denotes a lithium filled layer and $x$ is the fraction of $\mathrm{Li}$ in $\mathrm{Li}_{x} \mathrm{C}_{6}(0 \leq x \leq 1)$.

Li-GICs vary not only in their lithium concentrations, but also in their carbon stackings. The current consensus of all known stages, including their carbon stackings and lithium stoichiometries, is tabulated in Table 1.

Experimental observation of these stages relies largely on probing the average interlayer carbon spacing through diffraction measurements. Probing the lithium orderings of Li-

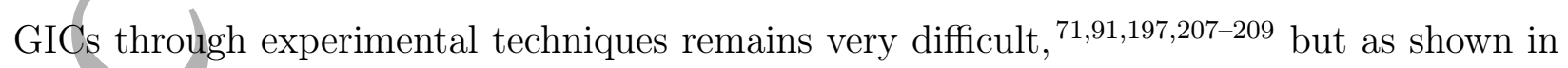
section 3.2.3, atomistic techniques shed light on these orderings. 
(b)

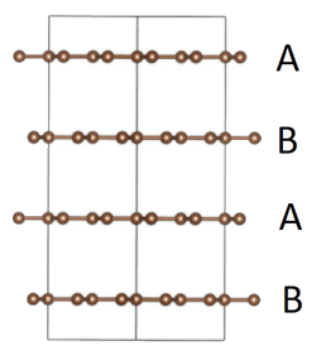

AB stacked graphite (a)

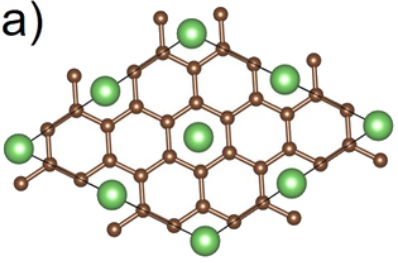

(c)

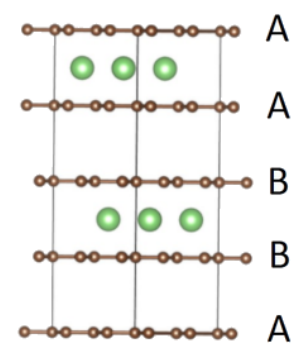

A (d)

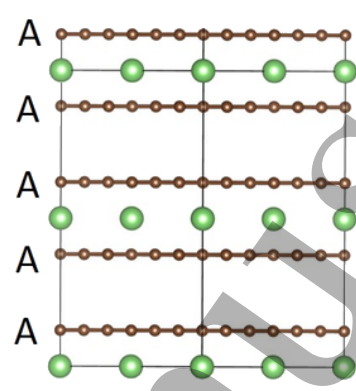

Stage II (e)

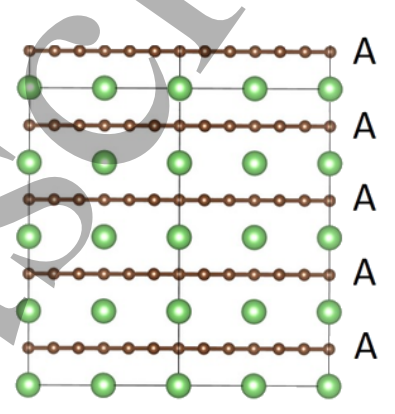

Stage I

Figure 7: Structural representations of different carbon stackings in experimentally confirmed stages of graphite. (a) Top down view of carbon and lithium arrangements in Stages I and II. (b-e): side views, showing the layers occupied with Li and carbon stackings in (b) empty $\mathrm{AB}$ stacked graphite, (c) $\mathrm{A} \alpha \mathrm{AB} \beta \mathrm{B}$ stacked dilute Stage II, with $\beta$ indicating a lithium layer translated with respect to $\alpha$, (d) A $\alpha A A \alpha A$ Stage II and (e) A $\alpha$ stacked Stage I. Green represent $\mathrm{Li}$ atoms, while the brown indicate $\mathrm{C}$ atoms. Reproduced from Ref. 71 - Published by The Royal Society of Chemistry.

Thermodynamic and kinetic properties of Li-GICs have been studied by considering various structures of $\mathrm{LiC}_{6 n}$ using Density Functional Theory (DFT), $\frac{70|76| 196|196| 210 \mid 216}{216}$ mean field, ${ }^{91 / 92 \mid 217}$ canonical and grand canonical Monte Carlo (MC), , ${ }^{76 / 218 \mid 219}$ and kinetic Monte Carlo (kMC) simulation techniques. ${ }^{76|102| 103|214| 220}$ The rest of the section outlines electronic structure based studies of experimentally measurable bulk thermodynamic properties, before describing atomistic modelling of kinetic properties.

\subsubsection{Equilibrium potential and measured open circuit voltage}

Knowledge of the correct phase behaviour of an intercalation electrode is an important pre-requisite to building a dynamic model of the intercalation process. One of the most 
Table 1: Overview of carbon stackings and stoichiometries of Li-graphite stages from the literature, where Latin characters denote carbon stackings and Greek characters denote Lifilled layers. $\frac{188|189| 195|197| 200 \mid 202+206}{2}$

\begin{tabular}{|ccc|}
\hline Stage & Stacking & $x$ in $\mathrm{Li}_{x} \mathrm{C}_{6}$ \\
\hline Stage I & $\mathrm{A} \alpha \mathrm{A} \alpha$ & $x=1\left(\mathrm{LiC}_{6}\right)$ \\
Stage II & $\mathrm{A} \alpha \mathrm{AA} \alpha \mathrm{A}$ & $x=0.5\left(\mathrm{LiC}_{12}\right)$ \\
Dilute Stage II (IID) & $\mathrm{A} \alpha \mathrm{AB} \beta \mathrm{B}$ & $x \approx 0.33\left(\mathrm{LiC}_{18}\right)$ \\
Stage III & $\mathrm{A} \alpha \mathrm{AB} / \mathrm{A} \alpha \mathrm{ABA} \alpha \mathrm{AC}$ & $x \approx 0.22\left(\mathrm{LiC}_{27}\right)$ \\
Stage IV & Unknown & $x \approx 0.17\left(\mathrm{LiC}_{36}\right)$ \\
Dilute Stage I (ID) & $\mathrm{AB}$ & $x \approx 0.083\left(\mathrm{LiC}_{72}\right)$ \\
Graphite & $\mathrm{AB}$ & $x=0$ \\
\hline
\end{tabular}

directly measurable observables is the experimental open circuit voltage (OCV), which is related to the equilibrium potential determinable from atomistic methods (c.f. Methods section 2.3.1). The OCV is an important input parameter in continuum models and is also used in control models, for example, to determine the state of charge of a battery within a Battery Management System (BMS). ${ }^{221}$ Inputting a polynomial fit to the experimental OCV at an arbitrary temperature without physical meaning could lead to incorrect predictions of temperature-dependent behaviour in these models. Therefore, to attain predictive, dynamic models on longer length scales, atomistic models of the OCV and equilibrium potential are important and can contribute to physically more robust and more predictive temperature dependence in continuum and control models. $\frac{9166}{960}$

In any intercalation electrode, ordered phases give rise to steps in the OCV. In the lithium-graphite system, the ordered stages described in section 3.2.1 therefore give rise to characteristic features in OCV versus $x$ curves ${ }^{195 \mid 200}$ as shown in Figure 8. The influence of the Li-graphite stages on the measured OCV at $T \approx 25{ }^{\circ} \mathrm{C}$ has been well charac-

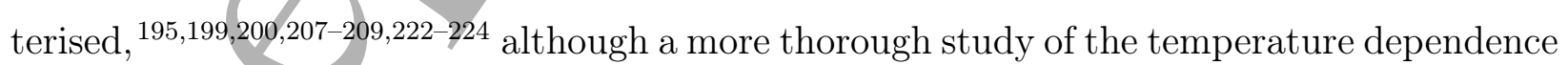
of the OCV has only been conducted more recently. ${ }^{71}$ Each OCV plateau represents a different two-phase equilibrium. At zero Kelvin, there is no contribution from configurational entropy and each step represents a sudden transition between two different two-phase equilibria. This is the behaviour that can be captured using DFT code. The cluster expansion 
framework, described in more detail in the Methods section 2.1.4, allows the accuracy of DFT to be retained to explore configurational degrees of freedom. Thermal fluctuations can be included by determining effective cluster interactions (ECIs) from fitting DFT data and using these as parameters within an MC method (section 2.1.5). The entropy contribution at temperature, $T>0 \mathrm{~K}$ has the effect of smoothing out those steps, $\frac{7191217225}{12}$ which is caused by some limited single phase solubility around the stoichiometric composition. This can be seen in experimentally measured OCV profiles at $T \approx 300 \mathrm{~K}$, such as the ones shown in Figure 8 .

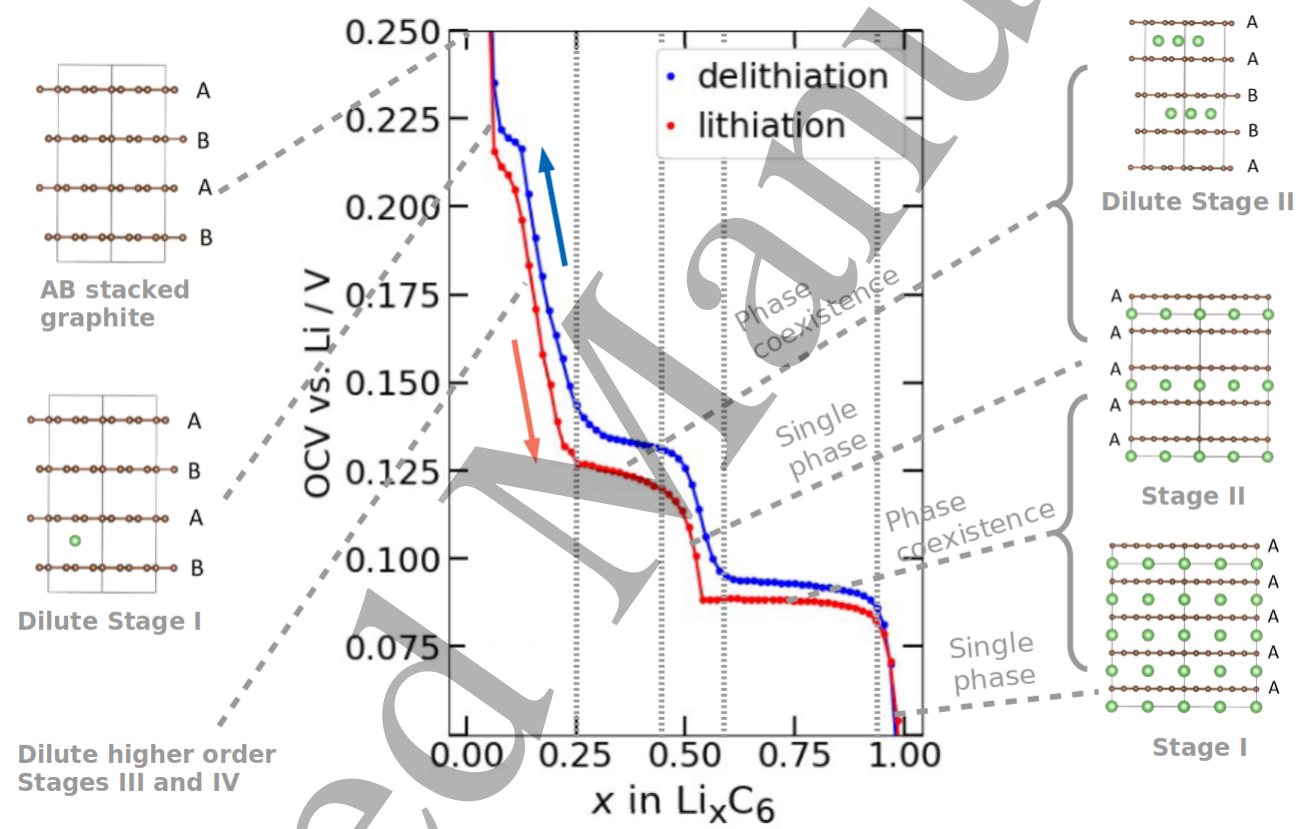

Figure 8: Illustration of OCV features of lithium in graphite using experimental data from ref. 91. Lithiation and delithiation behaviour is overlaid; labelled stages are linked to the lithiation profile, which is closer to the true equilibrium potential. Reproduced from Ref. 71 - Published by The Royal Society of Chemistry.

The equilibrium potential versus $x$ can be modelled through atomistic techniques. For example, Li-graphite phase diagrams were constructed and the equilibrium potential was modelled by Persson et al. ${ }^{[76}$ They performed a cluster expansion of Li degrees of freedom from total energy DFT calculations, by fixing the carbon stacking degrees of freedom. Those degrees of freedom represent the host lattice stackings in the experimentally confirmed stages shown in Figure 7. Typically, different cluster expansions are performed in Li-vacancy lattices 
of the respective hosts, ${ }^{71 / 76 \mid 196}$ to account for carbon stacking degrees of freedom with the result from a more recent work ${ }^{71}$ represented in Figure 9 a. Within this work, AA, AABB, and $\mathrm{AB}$ stackings of the host lattice were considered, representing all stages of order up to II (c.f. Figure 7). Reference states at $x=0$ and $x=1$ were used in AB and AA stackings, respectively, to linearly correct the free energy and thus obtain the formation energies at each lithium concentration. The convex hull over all stackings represents the lowest energy structure for a given $x$ value. A common tangent construction between the different stackings represents two-phase coexistence. The slope of the resultant ground state free energy profile, $d G(x) / d x$, (equation 29) equals the intercalated Li chemical potential, $\mu$, where $-\mu$ is equivalent to the equilibrium potential at $T=0 \mathrm{~K}$, as represented more generally in Figure 6 and the surrounding discussion in the Methods section.

The phase behaviour of the lithium-graphite system, and therefore the voltage profile, is sensitive to the vdW interactions between the carbon planes. $76 / 210 / 211$ Conventional DFT approaches without accounting for vdW interactions do not correctly reproduce the structure and energetics of graphite and Li-GICs ${ }^{76 / 2101211}$ (Figure 9p). Therefore, vdW-corrected DFT approaches, for example DFT-D2 $\frac{45}{4}$ and DFT-D3, $\frac{47}{2}$ are important for correctly describing the phase behaviour and dynamics of graphite and Li-GICs. Persson et al. considered the vdW interaction as a constant. ${ }^{76}$ This approximation can accurately describe the step height at $x=0.5$ (the height difference represents the difference between the chemical potentials in the Stage I-Stage II and Stage II-Stage IID coexistence regions). The simulated voltage profile Figure $9 \mathrm{~b}$ (blue line), shows that the constant vdW interaction results in a systematic error in the voltage scale.

Voltage profiles like the ones shown in Figure $9 \mathrm{~b}$ represent the ground state behaviour, at $T=0 \mathrm{~K}$. As an additional step, cluster expansions can be used to parameterise an MC simulation (section 2.1.5) and therefore include thermal fluctuations. The lithium-graphite phase diagram, Figure 9c, has been constructed by performing a combination of canonical and grand canonical MC simulations at different temperatures.76 

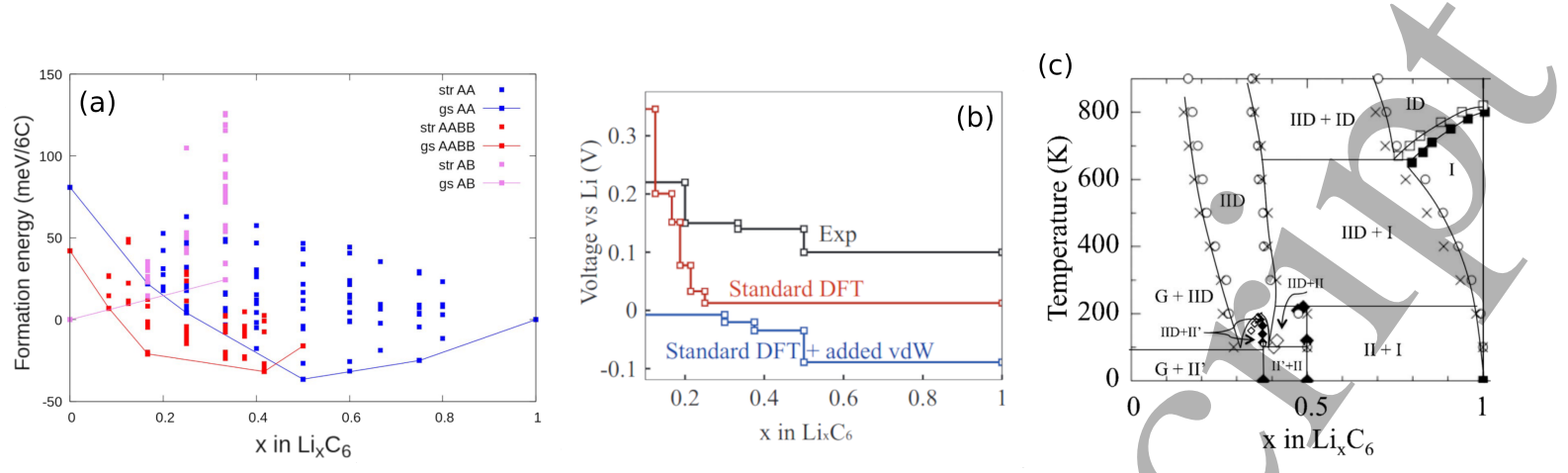

Figure 9: (a) Formation energies of lithium in graphite performed with different carbon stackings. All calculated structures are denoted "str" while the "gs" represent the ground state structures in each of the three carbon stackings: AB, AABB, AA. (b) Phase diagram of lithium in graphite, determined by performing Monte Carlo calculations parameterised by effective cluster interactions from Density Functional Theory calculations. (c) zero kelvin equilibrium potential profiles dependent on different levels of van der Waals corrections. (a) Reproduced from Ref. 71 - Published by The Royal Society of Chemistry; (b-c) Reprinted with permission from Ref. 76. Copyright 2010 American Chemical Society.

The experimental OCV and the theoretical equilibrium potential are often, erroneously, considered to be equivalent. However, the OCV refers to the measured cell voltage without any external current and drifts with time. With sufficient time, it is often assumed the OCV will eventually relax to the equilibrium potential, but meta-stable states can occur that show no variation over experimental time scales of hours or even days. ${ }^{71 / 2261227}$ The true equilibrium potential, as defined in equation 29, is a thermodynamic quantity and is not history-dependent. ${ }^{971}$ Experimentally, a hysteresis of the measurable OCV between lithiation and delithiation is observed for Li/graphite half cells $\frac{71|170| 195|200| 206|207| 223|228| 229}{\text { as }}$ shown in Figure 8. Hysteresis is observed even after several hours of relaxation time and for $T>298 \mathrm{~K}$, clearly demonstrating that the measured OCV is not a simple function of the thermodynamic ground state. Hysteresis therefore poses an interesting challenge to atomistic modellers.

It was recently shown that (de)-lithiation hysteresis in graphite is intimately connected with disorder in Stage II configurations and appears to be associated with a different carbon stacking pathway in each cycling direction. ${ }^{71}$ Notably, energetic barriers to translate between 
ground state configurations, as determined through climbing image nudged elastic band (CINEB) calculations (Methods section 2.1.3), do not explain the hysteresis in graphite. Nonground state configurations are involved in the delithiation direction. Understanding that behaviour requires the configurational entropy of Li/vacancy arrangements to be quantified, which is explained in more detail in the next section.

\subsubsection{Entropy}

The internal energy of intercalation electrodes arises largely from electrostatic interactions between the constituents. Those interactions can be well approximated by DFT. An atomistic description of the entropic behaviour of intercalation electrodes, $S(x)$, is also needed to correctly model thermal behaviour at $T>0 \mathrm{~K}$. The partial molar entropy, $d S(x) / d x$, is an experimentally accessible quantity, which can be probed by monitoring how the OCV, described in the previous section, varies with temperature (equation 31, c.f. refs. 71,91,93,100, 169, 170,230 for further details). $S(x)$ is a sum of configurational, vibrational, and electronic components. $\frac{169 \sqrt{225}}{}$ For lithium in graphite, the electronic component can be neglected and the vibrational component can be well approximated by assigning a Debye temperature to all of the vibrational modes, $\frac{169225}{2}$ or by computing phonon spectra from electronic structure

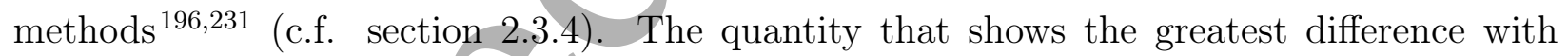
lithium concentration, $x$, is the configurational entropy of Li/vacancy arrangements, $S_{\text {config }}$. Because of the staging phenomena described in section 3.2.1, $S_{\text {config strongly deviates from }}$ ideal solid solution behaviour for Li in graphite.

The partial molar entropy $d S(x) / d x$ is difficult to interpret atomistically and so integration is required to get $S_{\text {config: }}$

$$
\int_{x^{\prime}=0}^{x^{\prime}=x}\left(\frac{\partial S_{\text {config }}\left(x^{\prime}\right)}{\partial x^{\prime}}\right) d x^{\prime}=S_{\text {config }}(x) \approx S(x)-S_{\text {vib }}(x)
$$

${ }_{1004}$ where $S_{\text {vib }}$ is the vibrational entropy approximated by Debye temperatures. $\frac{169[225}{125}$ The integration constant is $S_{\text {config }}=0$ at $x=0$, because there can be no Li disorder in pure 
graphite.
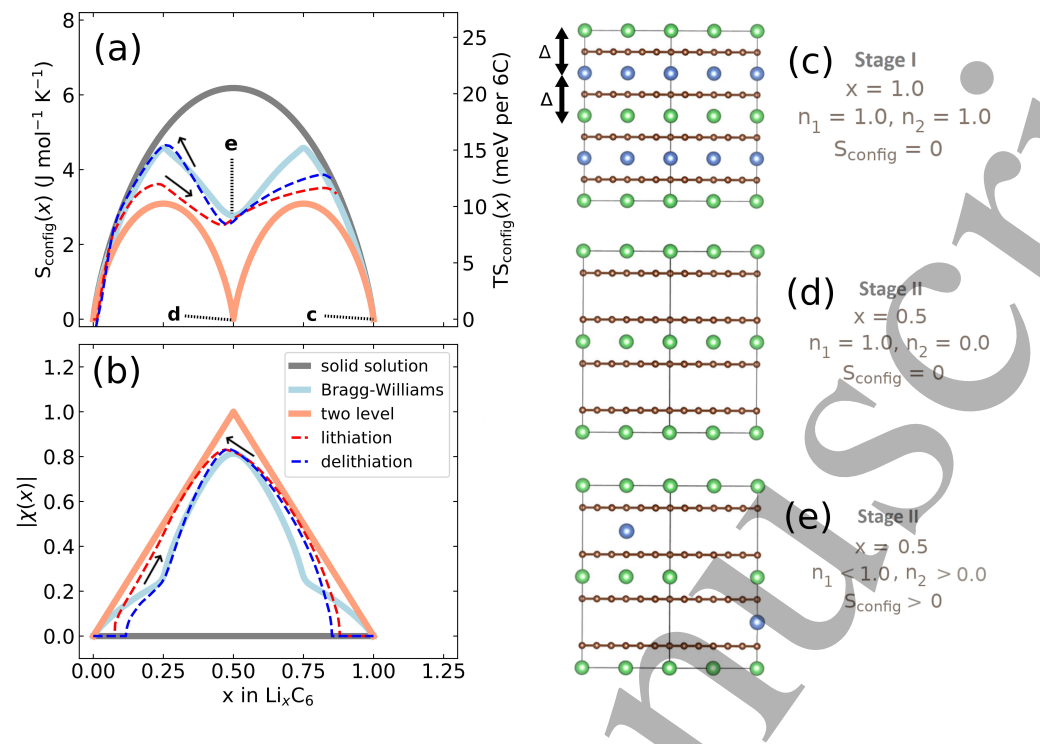

Figure 10: (a) Configurational entropy obtained at $T=320 \mathrm{~K}$ : dark grey solid line: ideal solid solution; light blue solid line: Bragg-Williams solution; orange solid line: sequential two level solid solution; red dashed line: experimental lithiation; blue dashed line: experimental delithiation. (b) Order parameter $|\chi|$, as described in the main text, labelled as in (a). In (a), select points (c-e) are indicated and schematic representations of the lattice occupations of $\mathrm{Li}$ in levels $n_{1}$ (green balls) and $n_{2}$ (blue balls) are shown on the right. Reproduced from Ref. 71 - Published by The Royal Society of Chemistry. 1018

Dashed lines in Figure $10 \mathrm{a}$ denote post-processed experimental data obtained during lithiation and delithiation using equation 42 from ref. 71. Qualitatively, this shows more configurational Li disorder, i.e. larger entropy, is obtained during delithiation than lithiation. The lithium arrangements can be split into sublattice occupancies $n_{1}$ and $n_{2}$ arranged in alternate planes, as shown visually in Figure 10 -e. Each sublattice occupancy is linked to the degree of lithiation, $x$, via $x=\left(n_{1}+n_{2}\right) / 2$.

Solid lines in Figure $10 \mathrm{a}-\mathrm{b}$ indicate three hypothetical cases. The orange solid line denotes solid solution (random) filling of Li into one of the sublattices for $x<0.5$, followed by solid solution filling of the other sublattice, resulting in two maxima. Note that $S_{\text {config }}$ is zero in Stage II at $x=0.5$ (c.f. Figure $10 \mathrm{~d}$ ). The dark grey line shows the result 
only nearest neighbour repulsive pairwise lithium interactions between planes of $\Delta=75$ $\mathrm{meV}$ and no in-plane interactions. That model allows a direct evaluation of the partition function (c.f. equation 10) by enumerating through all possible arrangements of Li atoms on the two sublattices for a given $x$ within the canonical ensemble. The out-of-plane LiLi interactions are treated within a mean field (non-local) approximation to simplify the computation (details and formulae in refs. 91,921217).

The Bragg-Williams model produces a behaviour in $S_{\text {config }}(x)$ between that expected for the solid solution and sequential two level filling. At $x=1$, there is a net repulsion on each $\mathrm{Li}$ atom of $2 \Delta$, as represented in Figure 10c. At $x=0.5$, one of the sublattices becomes preferentially filled, as represented schematically in Figure 10 e. In contrast, a perfect Stage II structure as predicted by sequential two level filling (Figure 10d), would result in $S_{\text {config }}(0.5)=0$.

These results can be understood within the framework of order parameters. ${ }^{81}$ The relevant staging order parameter, $\chi(x)=n_{1}-n_{2}$, is shown in Figure 10b. Formally, $\chi(x)$ takes values between -1 and +1 , but only the absolute value is meaningful in this case. If $|\chi(x)|=1$, then only one layer is filled with $\mathrm{Li}$, representing maximal staging order. If $\chi(x)=0$, both sublattices are occupied with equal probability, maximising disorder and hence no staging order is observed.

Greater interlayer Li disorder is observed during delithiation below $x=0.5$. The Li ordering, as described by the order parameter, closely follows the Bragg-Williams model. This is expected if the host lattice remains in a metastable AA stacking. The lithiation behaviour shows a configurational entropy closer to solid solution filling of half the sites, which would be expected in AABB stacking, since only half the interlayers (i.e. those locally adopting $\mathrm{AA}$ or $\mathrm{BB}$ stacking) provide favourable Li insertion sites. As shown in Figure $9 \mathrm{a}$, this is the ground state stacking configuration for $x<0.5$.

The wider implication of these results is that the transformations between the stackings in 1045 graphite, and possible stacking dynamics in other layered intercalation hosts, deserve more 

attention. These phase transformations not only create a challenge from a cell diagnosis point-of-view, they could also be partially responsible for mechanical degradation, fracture, unstable interfaces and loss of active material. Phase transformations should be described in a rigorous way in continuum models. It is not sufficient to approximate the guest ions as an ideal solid solution as, for instance, done in the popular Doyle-Fuller-Newman (DFN)-type models.

It is worth noting that the host lattice ordering of graphite obtained in electrochemical conditions is difficult to access from experimental analysis. In-operando x-ray and neutron diffraction experiments allow the interlayer carbon separation to be obtained. 189|195|200|204|206 However, it is extremely difficult to obtain the stacking displacements within each carbon layer, as the superstructure peaks associated with these displacements ${ }^{197}$ are weak and obscured by peaks from the current collector..$^{206 / 208 / 209}$ A reason as to why there is still some dispute in the literature regarding the Li-graphite stages of order greater than 2, as highlighted earlier in 3.2.1, is that these in-plane displacements cannot be completely elucidated. These displacements influence the lithium ordering in each atomic layer, which is even harder to determine than the carbon ordering and is currently not feasible even in neutron diffraction experiments. It is also not possible to determine this information through local probes such as nuclear magnetic resonance (NMR) and Raman scattering. ${ }^{222 \mid 232}$ It is only through the combination of atomistic and statistical mechanical models, together with entropy profiling measurements, that the proportion of lithium in the different layers in Stage II configurations could be quantified. ${ }^{71}$ With regard to systems other than lithium-graphite, atomistic techniques could enable lithium guest orderings to be quantified by means of order parameters, which, for the reasons explained above, is information inaccessible through experiment alone. Synergies between models of host and guest ion orderings with appropriate experimental characterisation will enable a new generation of modelling tools that can predict these phenomena with greater accuracy. 
1073

1074

1075

1076

1077

1078

1079

1080

1081

1082

1083

1084

1085 atoms.

\subsubsection{Ion diffusion in Li-GIC}

Having outlined the use of atomistic techniques to evaluate observable thermodynamic properties of anodes and, in particular, graphite, this section focuses on the computation of bulk dynamic properties by DFT and kMC approaches.

Li diffusivity is similar for stage I and stage II Li-GICs, $\frac{76}{6 i t h}$ the probable Li migration pathways for $\mathrm{LiC}_{6 n}$ illustrated in Figure $\left.11\right|^{210}$ These pathways were determined from DFT calculations within a CI-NEB approach. Here, Li diffusion across the graphite layers through a carbon hexagon hollow $(\mathrm{H})$ are denoted as the through-plane pathway. The in-plane or two-dimensional Li migration along the crystallographic ab plane occurs either by a bridge (B) migration pathway, where Li passes through a rectangle of carbon atoms of subsequent layers, or a top $(\mathrm{T})$ migration pathway, where Li passes in between two congruent carbon

Figure 11: Li migration pathway in $\mathrm{LiC}_{6}$. In the through-plane pathway, lithium migrates through a carbon hexagon hollow $(\mathrm{H})$ along the crystallographic c direction. The in-plane pathways are denoted as bridge (B) and top ( $\mathrm{T}$ ). Reprinted (adapted) with permission from Ref. 210. Copyright 2014 American Chemical Society.

Diffusion proceeds in the aforementioned through-plane pathways and in-plane pathways via the Frenkel and vacancy mechanisms, respectively. Thinius et al. showed that Li diffu- 
energy barrier. 210 The calculated activation energy for this migration pathway is extremely high $(8.00-8.23 \mathrm{eV})$, therefore, the Boltzmann probability for diffusion through pristine graphene planes is negligible at $T=300 \mathrm{~K}$. It is therefore likely that diffusion in the $c$ direction occurs via grain boundaries. $\frac{216}{2 n}$ contrast, the activation energy for Li diffusion in the crystallographic ab plane is much lower $(0.42-0.52 \mathrm{eV})$, showing that in Li-GICs, Li diffuses mostly within the intercalation layers. $\stackrel{210}{2 n}$ the literature, DFT-based theoretical investigations provide the same qualitative trends for ion diffusion mechanisms in Li-GICs and the calculated activation barriers vary slightly, but are within the same order of magnitude. $215|216| 234 \mid 235$

In order to gain insights into the Li diffusion process in graphite, far from equilibrium and under fast charging conditions, Garcia et al. simulated a range of compositions between stage I and IV, i.e. dilute Stage I. 211 Their study determined reduced activation barriers in the in-plane migration pathways $(0.2-0.32 \mathrm{eV})$, which is attributed to the presence of a higher number of electrons compared to $\mathrm{Li}^{+}$ions, occurring at the very beginning of the lithiation cycle during fast charging conditions. This extra charge increases the interlayer spacing in the diffusion layer and adjacent channels, increasing the Li diffusivity. $\frac{211}{2}$ Ji et al. investigated the anisotropic strain effects on lithium diffusion in graphite anodes using DFT and kMC simulations. ${ }^{214}$ According to their study, the activation energy for Li diffusion in unstrained $\mathrm{Li}_{x} \mathrm{C}_{6 n}$ is $0.48 \mathrm{eV}$. The tensile strain along the direction perpendicular to the graphite planes facilitates in-plane Li diffusion by reducing the energy barrier and vice versa. 214

Gavilán-Arriazu et al. have recently simulated the dynamic properties of lithium intercalation in graphite using $\mathrm{kMC} . \frac{102] 103 / 220}{.}$ These models considered exchange of Li with the solution on one side of a slab (Figure 12), with only interplanar Li transport allowed, based on the diffusion barrier arguments presented above. Energetic barriers for Li exchange into/out of the graphite were calculated assuming Butler-Volmer kinetics, based on experimental exchange current density data. Interplanar diffusion barriers were computed using random walk theory, based on experimental data in the dilute limit. Respective barriers of $0.655 \mathrm{eV}$ 
and $0.370 \mathrm{eV}$ for exchange and interplanar diffusion were obtained. This approach enabled the simulation of several different dynamic properties dependent on lithium concentration, $x, \frac{102102}{10}$ sweep direction, $\frac{102}{102}$ and temperature, $\frac{103}{10}$ with a few of these highlighted in Figure 13. Additionally, the importance of metastable Daumas-Hérold orderings in Stage II cønfigurations $^{220}$ and clogging of lithium at the interface ${ }^{102}$ leading to slow Li insertion kinetics were identified as important challenges limiting the kinetics of the lithium (de)insertion processes.

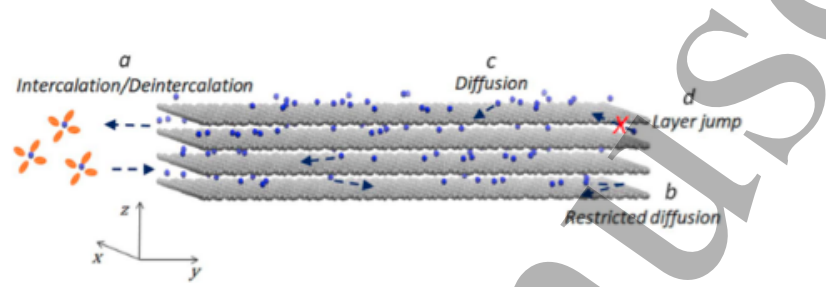

Figure 12: Representation of insertion and diffusion of lithium in graphite in a kinetic Monte Carlo model. Reproduced from Ref. 103 - Published by the/Journal of The Electrochemical Society.
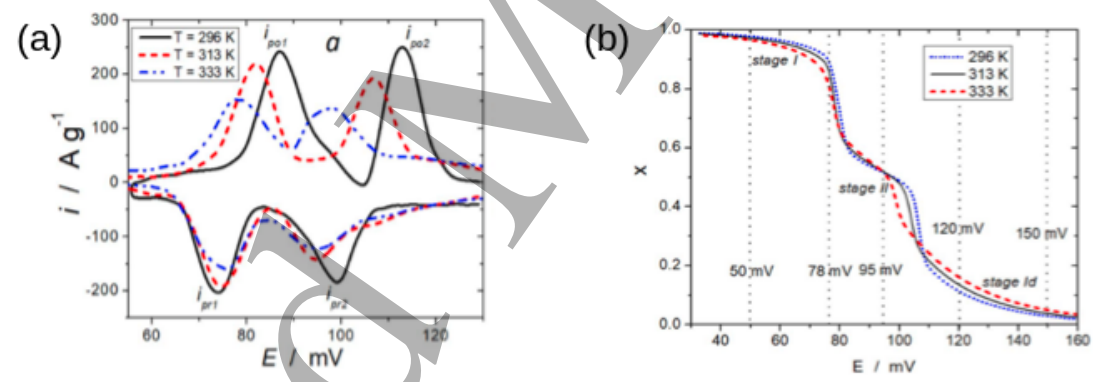

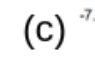

(c) ${ }^{-7.5}$

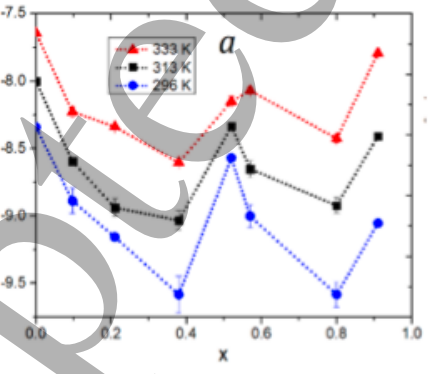

(d)

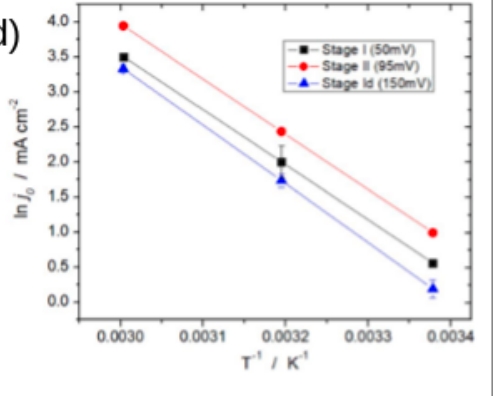

Figure 13: Effect of temperature on the dynamic behaviour of lithium insertion in graphite. (a) voltammograms (b) voltage profiles (isotherms) (c) diffusion coefficients, (d) exchange current density. insertion and diffusion of lithium in graphite in kMC model. Reproduced from Ref. 103-Published by the Journal of The Electrochemical Society.

Having described modelling of the thermodynamics and bulk Li diffusion in graphite, the 1123 following section will focus on another important aspect for a multiscale model: the structure 
1124

1125

1126

1127

1128

1129

1130

1131

1132

1133

1134

1135

1136

1137

1138

1139

and dynamics of the graphite edges.

\subsection{Graphite Surfaces and Interfaces}

\subsubsection{Possible graphite surfaces and their stability}

As discussed above, investigating the bulk properties of lithium is key to understanding Li intercalation kinetics and (dis)charging rates in graphite. However, Li exchange occurs between the graphite surfaces and the electrolyte, hence a multiscale model needs to include these phenomena. Addressing the surface properties of graphite would improve the understanding of (dis)charging behaviours at graphite anodes and possibly suggest how to enhance the (dis)charging rates.

As shown in Figure 14 and section 3.2, graphite consists of multiple stacked graphene layers. One of the exposed surfaces is the basal plane or the (001) surface, which has been widely investigated in both the theoretical and experimental studies. ${ }^{76[2101235+237} \mathrm{In}$ contrast, the non-basal planes attract less attention, due to their complicated edge morphology. Recently, experimental studies characterised the SEI formation and growth along the graphite edges as opposed to the basal plane, 2381239 indicating the importance of the graphite nonbasal plane for facilitating Li intercalation.
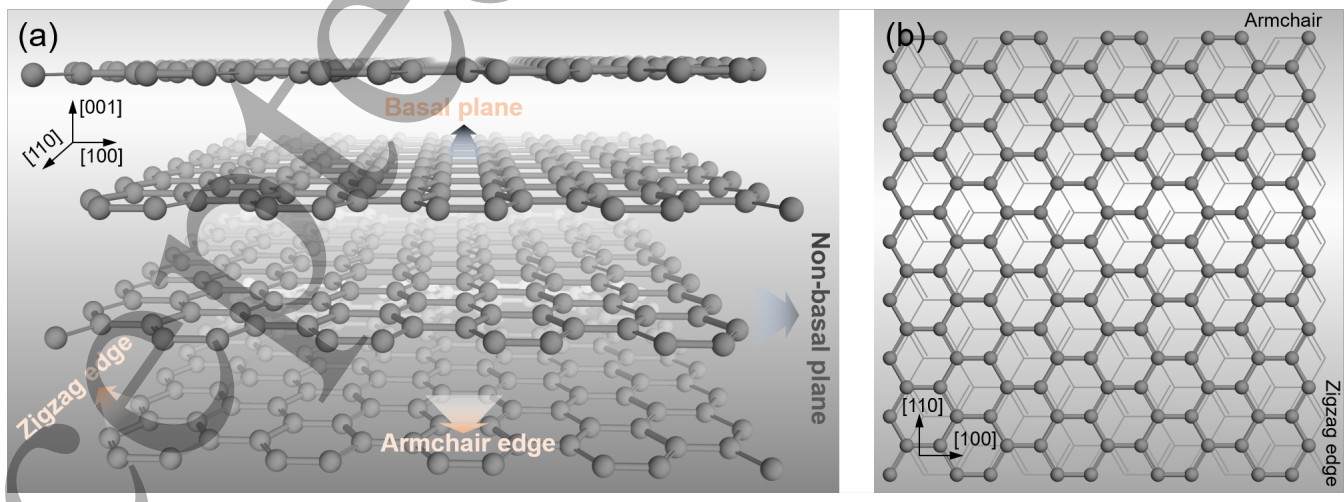

Figure 14: (a) structures of the basal plane and the non-basal plane of graphite. The latter plane consists of different edges of graphites, such as armchair edge and zigzag edge. (b) topological geometries of graphite edges. 
cluding the (001), (110), and (100) planes. The calculations were performed using dispersion corrected DFT approaches. $\stackrel{4648}{ }$ The surface energies of these planes were found to go in the

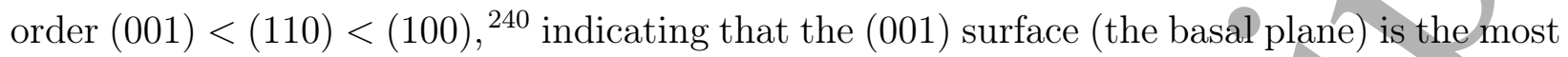
energetically favourable. However, this plane does not favour Li intercalation, due to the high diffusion barrier required for Li to go through the carbon hexagon, $\frac{76210}{21}$ as highlighted in the previous section on ion diffusion in Li-GICs ( $\sec 3.2 .4$. Li intercalation of graphite particles must therefore proceed either via defects in the (001) plane or via the non-basal planes.

The (100) surface consists of nanoribbons with a zigzag edge, whereas the (110) surface adopts an armchair conformation. The relatively unstable surface planes, such as the (100) plane, can be stabilised by various procedures, including chemisorption of oxygen atoms. $\frac{241}{24}$ It was found that the oxygen functional groups not only stabilise the graphite edges, but are also critical for the formation of the SEI layer near the edge, thereby preventing graphite exfoliation. ${ }^{242}$ Investigating those non-basal planes and their effects on Li intercalation are therefore important and are addressed in the following section.

\subsubsection{Surface Effect on Intercalation Energy}

Understanding the nature of $\mathrm{Li}$ intercalation in graphite is important for optimisation of the anode material. As described above, Li intercalation in the bulk of graphite has been widely

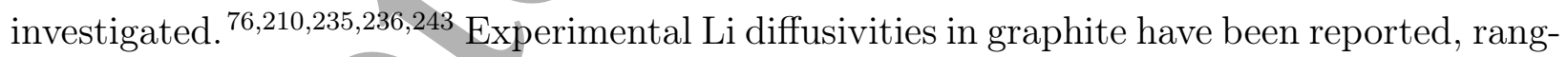
ing from $10^{-6}-10^{-14} \mathrm{~cm}^{2} \mathrm{~s}^{-1} \cdot \frac{235 \mid 244}{246}$ However, DFT calculations ${ }^{76}$ based on bulk graphite indicate that Li diffusion coefficients based on the AABB and AA stacked graphite are around $10^{-7} \mathrm{~cm}^{2} \mathrm{~s}^{-1}$ and decrease slightly with increasing Li concentration. $\frac{76}{76}$ The variability between reported experimental diffusion coefficients arises from a combination of the staging dynamics and the anisotropy of Li diffusion (through versus into the basal plane). There is also a difference between the surface morphologies of different types of graphite, i.e. the proportion of zigzag and armchair edges and their surface chemical terminations, implying 
(a)

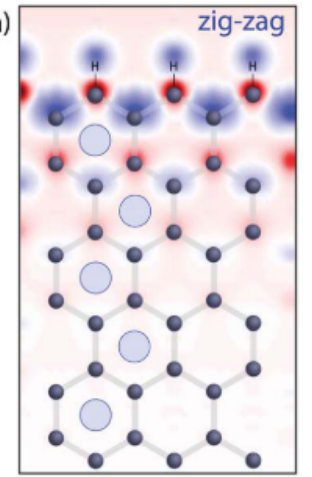

(b)

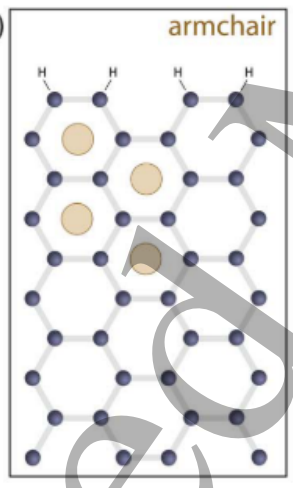

(c)

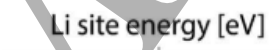

(d)

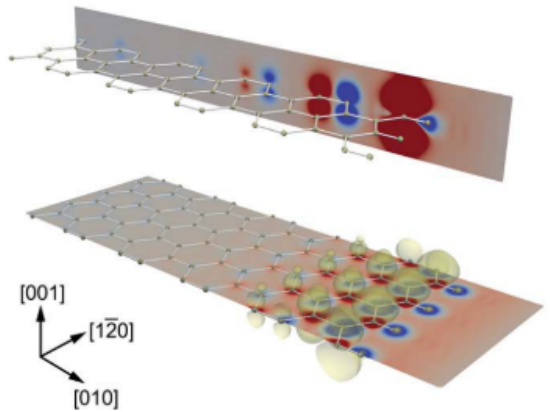

Figure 15: Structures of the zigzag-edged graphite (a) and the armchair-edged graphite (b). (c) shows the energy profile of Li adsorption in edged graphite. (d) is the spin densities of zigzag-edged graphite. The iso-surface value is 0.0002 e $\AA^{-3}$. Reproduced from Ref. 70 with permission from The Royal Society of Chemistry.

49
50
51
52
53
54
5
56
57
58
59

1179 From an atomistic perspective, the surface and edge morphology of anode materials were found to have a strong impact on Li binding energies. 247248 Through investigating Si 1181 nano-structures, Chan and Chelikowsky found that Li has higher binding energies at the 1182 bulk site compared to the edge, requiring a higher energy cost of Li migration from the ${ }_{1183}$ bulk towards the edge. ${ }^{249}$ In graphite anode materials, however, Leggesse et al. reported 
1207 1208 that the edged graphite systems showed remarkably enhanced Li binding energies and high Li mobility along graphite edges. .248 Peng et al. recently quantified the edge effects on Li intercalation in graphite. ${ }^{\frac{70}{1}}$ In their work, different edged graphites at dilute Li concentration were comprehensively investigated using DFT calculations. Interestingly, they found the unique topological electronic structures near the edges, particularly near the zigzag edge, induced distinct intercalation energies of Li in graphite. Figure 15 shows the Li adsorption energies at the armchair-edged and the zigzag-edged graphite, respectively. The adsorption energy, $E_{\text {ads }}$, is expressed as:

$$
E_{\text {ads }}=E_{\mathrm{Li} \mid \text { Graphite }}-E_{\text {Graphite }}-E_{\mathrm{Li}}
$$

where $E_{\mathrm{Li} \mid \text { Graphite }}, E_{\text {Graphite, }}$, and $E_{\mathrm{Li}}$ are the energies of Li adsorption in graphite, the pristine graphite, and one $\mathrm{Li}$ in body-centred cubic (bcc) Li metal, respectively. At the armchair edge, from the energy profile (c.f. Figure 15), the adsorption energy of Li is the lowest at the edge site $(-0.38 \mathrm{eV})$. With Li penetrating into the bulk, the adsorption energy decreases rapidly to $-0.24 \mathrm{eV}$ at the sub-surface site and becomes $-0.26 \mathrm{eV}$ at the bulk site. The topological geometry of the armchair edge promotes Li adsorption relative to the graphite bulk.

At the zigzag edge, the edge effect becomes even stronger, due to the existence of the surface state which consists of $\mathrm{C}-p_{\mathrm{z}}$ orbitals emerging from the zigzag edge. ${ }^{2501251}$ Figure 15. shows that $\mathrm{Li}$ achieves a much lower adsorption energy of $-0.66 \mathrm{eV}$ at the zigzag edge site, indicating the strong binding of $\mathrm{Li}$ at the edge. The edge effect in the zigzag system is much stronger thân that at the armchair edge and additionally penetrates into the bulk, indicated by the gradual decrease in magnitude of the Li adsorption energy from the edge to the bulk.

The zigzag edge displays completely different spin densities contributed by the $p_{z}$ orbitals perpendieular to the graphene planes, as shown in Figure 15a-b. ${ }^{70 \mid 24812501251}$ These spin densities consist of the unpaired electrons accumulating on the edged carbons. The amplitude 


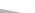

1234

of this topological surface state gradually diminishes over a few bond distances beneath the surface. It is this surface state that interacts with $\mathrm{Li}$ at the zigzag edge and favours its adsorption. In summary, the graphite edges show stronger interactions with Li than those in the bulk. The effect is especially pronounced at the zigzag edge, strongly stabilising Li binding due to the topological surface states.

\subsubsection{The Surface Effect on Li Diffusion}

As Li obtains higher binding energies at the graphite edge, due to the specific topological structure of graphite edges, $\underset{70248}{ }$ it's worth examining the impact of those edges on Li diffusion. In bulk graphite, the diffusion barrier of Li jumping from one site to another is around $0.4 \mathrm{eV}$ at the dilute limit. ${ }^{210} \mathrm{Li}$, however, exhibits completely different diffusion kinetics at graphite edges in contrast to those in the bulk.70248

Peng et al. show the energy profile of Li diffusion from the graphite edge towards the bulk at dilute Li concentration, Figure 16. In the armchair-edged graphite, Li has to overcome an energy barrier of $0.43 \mathrm{eV}$ to move from site 1 to site 2 and a $0.42 \mathrm{eV}$ barrier to further move from site 2 to site 3 . The direct jump from site 1 to site 3 has to overcome an energy barrier of $0.58 \mathrm{eV}$ and is therefore less favourable. In contrast, for bulk diffusion, Li needs to overcome a $\sim 0.43 \mathrm{eV}$ barrier to move to either adjacent site. The higher diffusion barrier at the armchair edge is caused by the compensation of Li adsorption energy at the edge site. At the zigzag edge, Li obtains two different diffusion pathways. Li diffusion from the edge (site 1) to the subsurface (site 3), where the diffusion barrier is $0.48 \mathrm{eV}$. In contrast, there is only a $0.21 \mathrm{eV}$ activation barrier for Li diffusion along the edge sites (site 1 to site 2), which is much lower. This indicates that $\mathrm{Li}$ is extremely mobile at the zigzag edge, which can be verified by the stronger flux connecting the edge sites compared to diffusion towards the bulk (c.f. Figure 16). Due to the surface effect identified at the zigzag edge, Li favours diffusion along the edge direction within the first sub-surface sites, as the diffusion barrier $(0.41 \mathrm{eV})$ is still lower than the barrier to moving Li into the bulk $(0.49 \mathrm{eV})$. Markov chain analysis 

was conducted in Peng et al.'s study to examine Li diffusion from the armchair edge and the zigzag edge to a bulk site $20 \AA$ below the edge surface (see Figure 16c). They demonstrated that Li diffusion from the armchair edge to the bulk site is around one order of magnitude faster than its diffusion from the zigzag edge to the bulk, due to the strong binding of Li at the zigzag edge that generates a deep potential well for Li. ${ }^{70}$

On the basis of these studies, it was shown that the graphite edges have strong effects not only on the Li intercalation energies but also on its diffusion kinetics close to the edge. ${ }^{701248}$ The effect is pronounced at the zigzag edge. 24212521253$]$ Thus much more sluggish (de)intercalation kinetics are expected at that edge, compared to the armchair edge. Strategies including promoting growth of armchair edge over zigzag edge during synthesis of graphite nanomaterials, $\stackrel{242}{2}$ and tuning the edge properties by chemical doping to improve Li diffusion rate towards the bulk could be useful to enhance Li (dis)charging rate for graphite anodes. $254 \sqrt[256]{256}$

Designing edge-controlled graphite for validating the electronic and electrochemical properties predicted by DFT is still state-of-the-art. Commercial graphite powders contain a distribution of sizes, the proportion of edge types on each particle is dependent on particle size, and it is currently not possible to form graphite with only one type of edge. This makes systematic experimental characterisation to determine the influence of edges difficult. ${ }^{257}$ To try to understand these effects Bernardo et al. studied the effect of hydrogen and oxygen gas etching on graphite materials with a higher incidence of zigzag or armchair edge orientations. ${ }^{242}$ The proportion of each edge was quantified by high resolution transmission electron microscopy (HR-TEM), with the authors finding that a higher proportion of zigzag edges leads to a less stable SEI. Velický et al. studied the local electron transfer rate, double layer capacitance, and local density of states of the edge versus the basal plane of graphite in a mierodroplet electrochemical cell. ${ }^{252}$ This study was feasible owing to the vastly different electronic structure and electron transfer rate constant of the basal plane versus the graphite edges. It has yet to be determined if it is currently feasible to distinguish these properties 
for the zigzag and armchair edge, in a real electrochemical environment, through such an approach. Even so, the finding that the surface state from the zigzag edge could be a major bottleneck to the Li intercalation rate is a triumph for atomistic modelling that could direct future materials design strategies and experimental characterisation for anodes. ${ }^{70258}$ Moreover, the interplay between edge orientation and nitrogen/boron doping warrants further systematic exploration from both experiment and theory.

These studies can also offer some universal insights for investigating the interface effects of other materials such as the cathode. Prior to Li intercalation into graphite, the Li desolvation process is also an important step affecting the overall (dis)charging rate. However, due to the complicated solid-liquid interface, addressing the graphite interaction with the electrolyte is an extremely challenging aspect for both modelling and experiment, as discussed in the following sections. We discuss the effect of that interface on Li plating and aspects related to the SEI in the following sections.

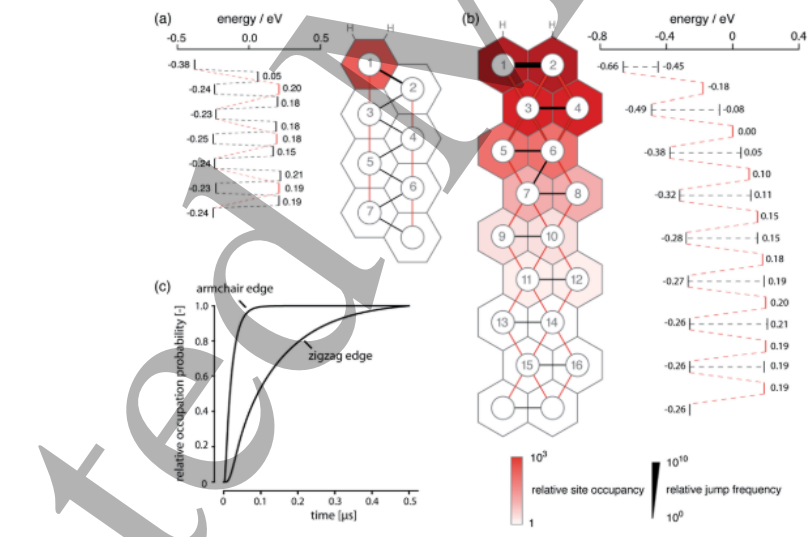

Figure 16: Li diffusion at (a) the armchair-edged and (b) the zigzag-edged graphite. The hexagons indicate lattice sites and the colours show occupancy probability relative to that in the bulk. The width of the lines connecting sites implies the jump frequencies. (c) shows the occupation probability for Li to occupy a site approximately $20 \AA$ below the graphite edge, relative to the steady-state value after being introduced at time zero at the edge. Reproduced from Ref. 70 with permission from The Royal Society of Chemistry. 
1275

1276

1277

1278

1279

1280

1281

1282

1283

1284

1285

1286

1287

1288

1289

1290

1291

1292

1293

1294

1295

1296

1297

1298

1299

1300

1301

\subsubsection{Li deposition on graphite anodes}

Apart from intercalation of $\mathrm{Li}$ ions into the graphite anode, $\mathrm{Li}$ ions can also deposit on surface of graphite in the form of metallic Li dendrites, which can grow during battery operation and cause internal short-circuits. Several situations for the deposition of Li metal on the graphite anode have been identified, as shown schematically in Figure $17{ }^{259} \mathrm{~A}$ "normal" intercalation mechanism is shown in Figure 17a. When the voltage of the graphite electrode drops below $0 \mathrm{~V}$ with respect to $\mathrm{Li} / \mathrm{Li}^{+}$, deposition of $\mathrm{Li}^{+}$ions on the graphite surface, as metallic $\mathrm{Li}$, becomes thermodynamically possible, as shown in Figure 176 . The thermodynamic criterion can be satisfied when the overpotential, $\eta_{i n t}$, is larger than the equilibrium voltage of the stage II to stage I phase transition $(\sim 85 \mathrm{mV})$. Deposition becomes kinetically feasible when the overpotential for the intercalation reaction $\left(\eta_{\text {int }}\right)$ becomes larger than the intercalation voltage $(\sim 85 \mathrm{mV})$, so that the graphite voltage drops below $0 \mathrm{~V}$ with respect to $\mathrm{Li} / \mathrm{Li}^{+}$. The overpotential originates from mass transfer limitations in the electrolyte region near the graphite edge, as shown schematically in Figure 17k. Li plating can be triggered upon local salt depletion in the electrolyte, $c_{l} \rightarrow 0$, if liquid diffusion is slow compared to intercalation. Solid-state diffusion between the graphite edge and the bulk, as shown schematically in Figure $17 \mathrm{~d}$, also contributes to this overpotential. Li plating can occur when intercalated $\mathrm{Li}^{+}$ions saturate the graphite edge $(c \rightarrow 1)$ and block further insertion, if diffusion from surface to the bulk is slow compared to Li insertion at the edge. A combination of both effects can result in Li deposition on the graphite surface.

A recent DFT study by Peng et al. has shown that in a vacuum environment: (1) Li deposition is more favourable near the graphite edges rather on the basal plane, (2) the energy barrier for Li deposition at the zigzag edge (only) increases with the degree of lithiation of the graphite, (3) chemical doping of nitrogen can increase the energy barrier and can possibly suppress the Li deposition on graphite anode on the zigzag edge. ${ }^{258}$ More advanced models for DFT simulations in the presence of an electrolyte under applied potential (cf. (sec. 2.2.1 and Ref. 260) ), have the potential to shed more light on the Li deposition phenomenon in 
experimental conditions.

A

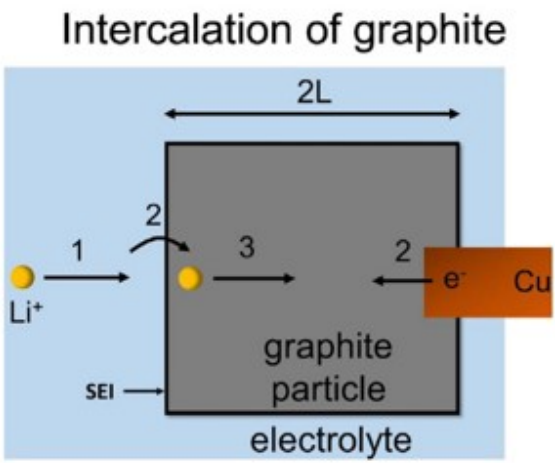

C

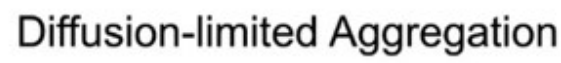

B

Thermodynamic condition

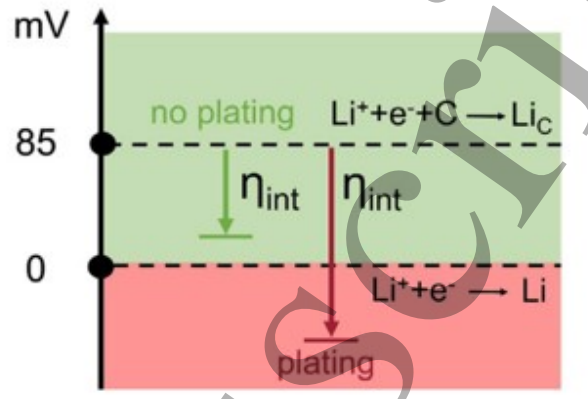

D Solid diffusion limitation

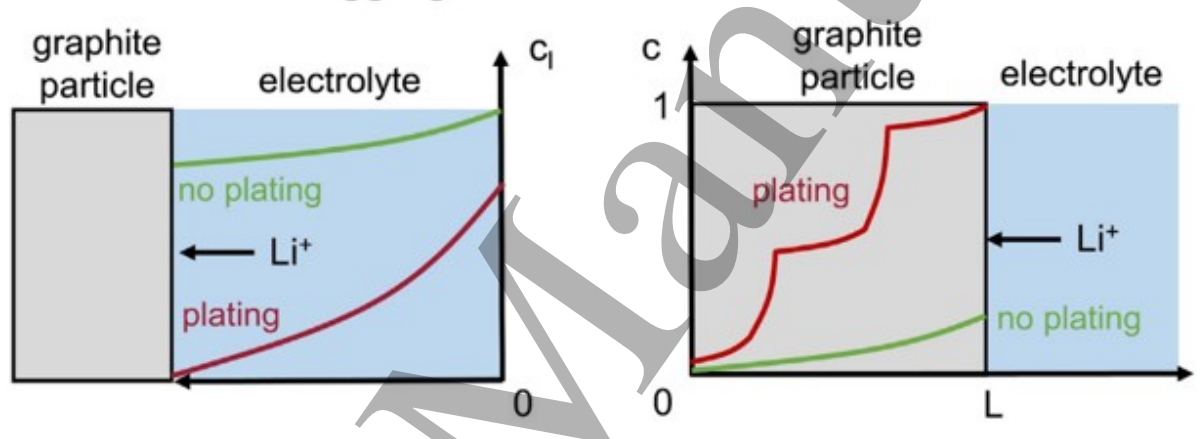

Figure 17: (a) 2D schematic of intercalation of a graphite particle. Three sequential steps take place during charging at the graphite anode: (1) $\mathrm{Li}^{+}$transport in electrolyte toward the reaction site; (2) $\mathrm{Li}^{+}$intercalation into a graphite particle (including de-solvation and migration through the SEI); and (3) $\mathrm{Li}^{+}$solid diffusion within the graphite particle. (b) Thermodynamic criterion for $\mathrm{Li}$ plating (cell voltage, $U<0 \mathrm{~V}$ versus $\mathrm{Li} / \mathrm{Li}^{+}$). The green and red arrows illustrate the required overpotentials to drive the insertion reaction at small current/fast insertion kinetics and large current/slow insertion kinetics. (c) 1D schematic of diffusion-limited aggregation resulting from electrolyte transport limitations. The green and red curves illustrate the $\mathrm{Li}^{+}$salt concentration profile in the electrolyte. (d) 1D schematic of solid diffusion-limitation mechanism. The green and red curves illustrate the $\mathrm{Li}^{+}$concentration profile in the graphite particle. Reprinted from Ref. 259, with permission from Elsevier.

\subsubsection{Solid-Electrolyte Interphase}

1304

The SEI is an important component of the rechargeable Li-ion battery and is formed from

1305 deposition of the decomposition products of the electrolyte and solvent on the anode surface. 1306 The SEI allows transport of $\mathrm{Li}^{+}$ions but blocks the transfer of electrons, thereby stopping 
further electrolyte decomposition reactions. $\frac{261 \mid 262}{2}$ Here we discuss aspects of the SEI related to our discussion of Li-ion diffusion energy barrier in bulk and graphite surfaces. A recent comprehensive review on the atomistic modelling of the SEI describes several other aspects of the SEI in detail: 16

- Electrolyte and solvent reduction mechanisms, including: prediction of the reduction voltage for each solvent and electrolyte species, the effect of the electrolyte solvation structure, the effect of anode surface termination, and the dynamic buildup of the nanometer thick SEI layer.

- Modification of the SEI by electrolyte additives and prediction of new electrolyte additives.

- Correlation of the SEI properties with battery performance, including: the electron insulating properties of the inorganic components in the SEI, the ionic conductivity of the SEI components, Li-ion desolvation at the SEI/electrolyte interface, chemical stability of the SEI components, and mechanisms of SEI growth and battery aging.

- The use of coatings to artificially design the SEI.

One way to describe the SEI is via the implicit continuum models described in sec. 2.2.1 Applying their DFT + implicit electrolyte model on an armchair edge of 1634-atom graphite slab in contact with a $0.5 \mathrm{M} \mathrm{LiPF}_{6}$ in EC solution, Dziedzic et al. calculated that a Li atom is $2.34 \mathrm{eV}$ more stable at the graphite edge than in the electrolyte solution. ${ }^{135}$ Similarly, Haruyama et al. found favourable energetics for Li intercalation from the electrolyte solution into the graphite edge. ${ }^{263}$ They also studied the variation in energy as a function of Li distance from the graphite edge, as shown in figure 18. In Haruyama et al.'s model, Li intercalation is accompanied by an electron gain from the external circuit. This was implemented using a grand canonical version of electronic DFT, where the number of electrons in the electrode can change subject to fixed electrode potential. Correspondingly, the appropriate thermodynamic quantity to represent this ensemble is the grand potential, $\Omega=A-\mu_{e} N_{e}$, 
which is plotted on the y axis for several different constant chemical potentials of electrons, $\mu_{e}$. Two illustrative cases include: (a) the potential of zero charge (PZC), which is the electrochemical potential of a charge-neutral Li-graphite system, and (b) the equilibrium potential (c.f. sections 2.3.1 and 3.2.2), where the net change in the grand potential for the intercalation reaction becomes zero. Haruyama et al.'s simulations estimate an energy barrier of around $0.6 \mathrm{eV}$ for $\mathrm{Li}$ intercalation into the graphite edge, which is close to the experimental measurements from impedance spectroscopy. $\frac{264}{26}$

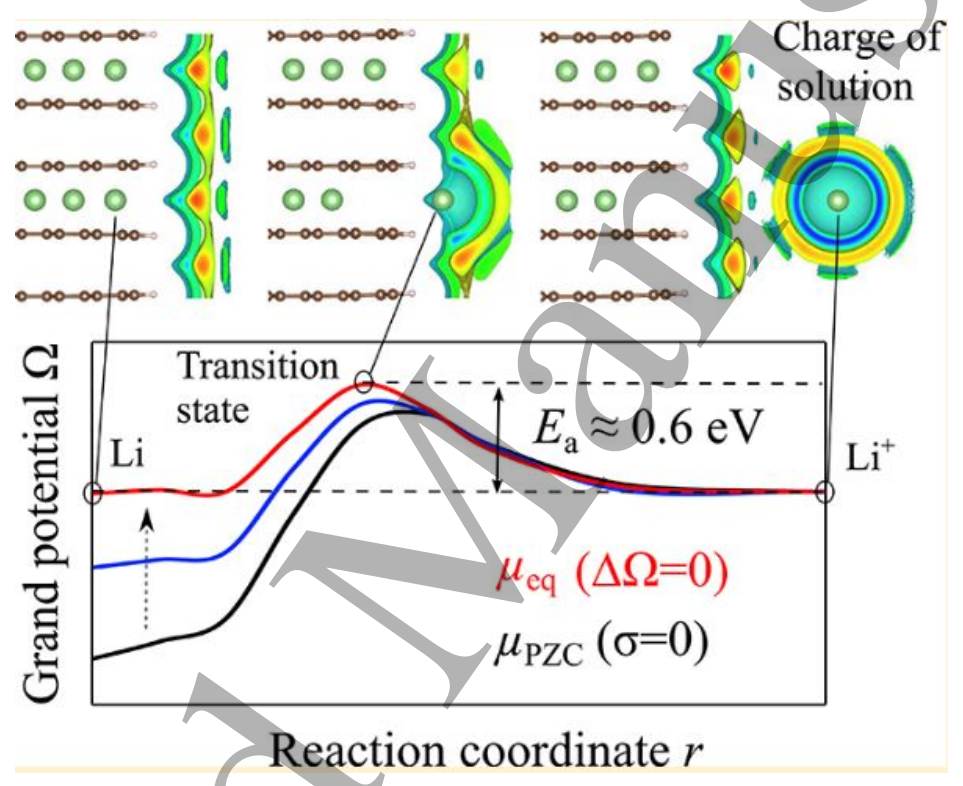

Figure 18: Profiles of grand potential $\Omega$ as a function of the Li-position during Li-intercalation process at the interface between graphite edge and an implicit electrolyte solution. The simulation is performed at conditions of constant chemical potential of electrons $\mu_{e}$ (constant electrode potentials similar to experiments). Reprinted with permission from Ref. 263. Copyright 2018 American Chemical Society.

Another way to describe the SEI is via explicit consideration of SEI components. Shi et al. performed a direct calculation of Li-ion transport in the $\mathrm{Li}_{2} \mathrm{CO}_{3}$ component of the SEI, 265 via DFT-based CI-NEB calculations (section 2.1.3). Two mechanisms for $\mathrm{Li}^{+}$diffusion were considered, namely, the knock-off and direct hopping mechanisms, which were found to have energy barriers of $0.31 \mathrm{eV}$ and $0.54 \mathrm{eV}$ respectively, as shown in Figure 19. The Li self-diffusion coefficient was calculated to be $1.1 \times 10^{-7} \mathrm{~cm}^{2} \mathrm{~s}^{-1}$ and $8.4 \times 10^{-12} \mathrm{~cm}^{2} \mathrm{~s}^{-1}$ respectively. Estimating the formation energy of corresponding defects in the lattice of 
$\mathrm{Li}_{2} \mathrm{CO}_{3}$ as a function of voltage, the total activation energy barrier for Li-ion diffusion was predicted to be in the $0.67-1.07 \mathrm{eV}$ range for the knock-off mechanism and in the $0.92-1.32$ $\mathrm{eV}$ range for the direct-hopping mechanism.
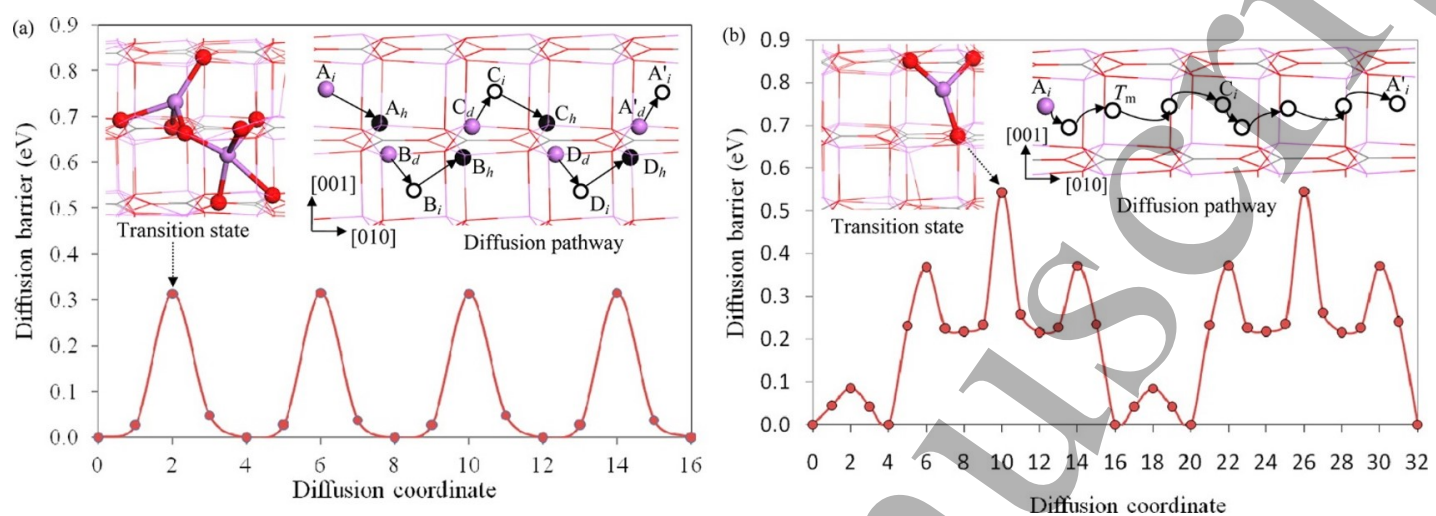

Figure 19: Energy barrier for Li-ion transport in the SEI via (a) knock-off and (b) direct hopping mechanisms. Reprinted with permission from Ref. 265. Copyright 2012 American Chemical Society.

The predicted values of the Li-ion diffusion energy barrier by both the implicit and the explicit models described above are significantly higher than that in the bulk of graphite, which is reported to be between $0.2-0.5 \mathrm{eV}$ (c.f. section 3.2.4). limiting role of the SEI in determining overall kinetics of Li-ion diffusion and the overall rate-capability of Li-ion batteries.

\section{$3.4 \mathrm{C} / \mathrm{Si}$ composities}

Use of anode materials capable of electrochemically alloying with lithium could allow higher energy densities than are possible with graphite. In particular, silicon, due to its high gravimetric capacity of $4200 \mathrm{mAh}^{-1}$, has achieved tremendous attention as an anode material. ${ }^{266} \mathrm{Si}$ has a low electrochemical potential $0.37-0.45 \mathrm{~V}$ vs. $\mathrm{Li} / \mathrm{Li}^{+}$, which is only $\sim 0.27 \mathrm{~V}$ higher than graphite. ${ }^{267} \mathrm{Si}$ is highly abundant, cost effective and non-toxic. ${ }^{267+269}$ While pure Si anode materials are not presently viable, present day anode materials combine a small atomic fraction (typically 5-10 at \%) of silicon with graphite to boost the gravimetric capacity of the anode. ${ }^{14}$ However, there are certain challenges in understanding the behaviour 

of $\mathrm{Si}$ and $\mathrm{C} / \mathrm{Si}$ composites that are summarised in this section.

The phase diagram of lithium and silicon shows five crystalline intermetallic Zintl-like phases: $\mathrm{Li}_{21} \mathrm{Si}_{5}, \mathrm{Li}_{13} \mathrm{Si}_{4}, \mathrm{Li}_{7} \mathrm{Si}_{3}, \mathrm{Li}_{12} \mathrm{Si}_{7}$, and $\mathrm{LiSi} . \stackrel{270}{ }$ However, $\mathrm{LiSi}$ is not accessible under electrochemical conditions, since it is synthesised under high pressure, and the stoichiometry of $\mathrm{Li}_{21} \mathrm{Si}_{5}$ is disputed, with a mixed $\mathrm{Li}_{21} \mathrm{Si}_{5} / \mathrm{Li}_{22} \mathrm{Si}_{5}$ phase also proposed. ${ }^{271}$ Under electrochemical conditions, metastable phases with compositions $\mathrm{Li}_{15} \mathrm{Si}_{4}{ }^{272}$ and amorphous $\mathrm{Li}_{x} \mathrm{Si}_{y}$ can be formed. ${ }^{273}$ It has been proposed that a different reaction pathway between these phases during lithiation and delithiation contributes to the observed charge/discharge hysteresis in lithium silicides and $\mathrm{C} / \mathrm{Si}$ composites. ${ }^{272} \mathrm{In}$ particular, Jiang et al. found that the crystalline phase $\mathrm{Li}_{15} \mathrm{Si}_{4}$ is accessed during lithiation, but the lattice undergoes an amorphisation process during delithiation, with the latter step being rate determining. ${ }^{272}$ This limits the utility of ground state DFT calculations for understanding the Li-Si system under operating battery conditions, and is therefore a challenge for multiscale modelling.

An additional challenge is the volume expansion. Upon full lithiation, the volume of $\mathrm{Si}$ can expand to more than three times its original volume, which means the Si electrodes do not retain their morphology during prolonged cycling or, even worse, some particles become detached from the electrode assembly. ${ }^{267269274}$ This volume expansion/contraction during cycling also leads to severe cracking and degradation of the SEI. It is for mainly these reasons that pure $\mathrm{Si}$ anodes are not currently commercially viable and must be combined with graphite. Several strategies have been proposed to change the morphology to mitigate these issues, including development of different Si robust nanostructures (0D or hollow nanoparticles, 1D nanowires, 2D film-like $\mathrm{Si}$, and 3D Si structures), ${ }^{267}$ and the development of composites ( $\mathrm{Si} /$ carbon composites, $\mathrm{Si}$ /polymer composites, $\mathrm{Si}$ alloys, and $\mathrm{Si} /$ metal oxide composites) .14 While modelling the complex nature of the degradation pathways of the $\mathrm{Si}$, Si-composites and their SEIs is presently out of reach of atomistic methods, these techniques nonetheless emerge as natural tools for high-throughput screening of different promising anode materials. ${ }^{275}$ These approaches can also tell experimentalists the most promising part 

Many aspects of modelling the bulk behaviour of lithium (de)insertion graphite are well

of the parameter space in which to perform more extensive, time consuming, and sometimes costly characterisation.

A more comprehensive overview of the application of mesoscale models to challenging composite systems is presented by Franco et al., with the volume averaging approach highlighted perhaps being particularly applicable to $\mathrm{Si}$ and $\mathrm{C} / \mathrm{Si}$ systems. ${ }^{233}$ Particularly for carbon anodes in combination with $\mathrm{Si}$ or silicon suboxide $(\mathrm{SiOx})$, collectively referred to as $\mathrm{C} / \mathrm{Si}$ or $\mathrm{C} / \mathrm{SiO}_{x}$, it may presently be necessary to sacrifice some details of the atomic level description to enable these systems to be tractably modelled at either mesoscale or continuum levels. Regarding the dynamic and metastable behaviour described above, kMC would be a natural technique to bridge length scales and include different time scale dynamic events, as explained in a recent review dedicated to this technique. ${ }^{104}$

\subsection{Outlook and challenges for anodes}

Graphite remains the predominant anode material in most Li-ion cells, due to its suitably high capacity of $372 \mathrm{mAh} \mathrm{g}^{-1}$, an operating potential close to $0 \mathrm{~V}$ vs. $\mathrm{Li} / \mathrm{Li}^{+}$, and its compatibility with liquid organic electrolytes. Alternative materials that form solid solutions with lithium (including silicides) presently do not have sufficient long term structural stability to be used as the primary anode material, requiring them to be composites with graphite. The development of graphite-based anodes has relied upon not only understanding staging formation in bulk, but also upon the development and understanding of a stable SEI and its implications of that SEI for cell longevity and (de)intercalation rate behaviour.

Advancements in developing all solid-state batteries (ASSBs) have resulted in additional research of Li metal anodes, as reviewed by Fang et al. ${ }^{276}$ and Li et al. ${ }^{277}$ In this section, we have summarised the safety and degradation challenges caused by lithium plating on graphite anodes. The use of Li metal as the anode for LiBs and ASSBs still face similar issues regarding redeposition of metallic $\mathrm{Li}$ as dendrites and consumption of cyclable lithium. .2762277 

understood. As shown in this section, challenging aspects like quantifying the Li ion ordering with lithiation fraction can only be obtained by combining experimental observations with atomistic models. However, there are challenges with atomistic modelling in anodes that hinder improvements in capacity, rate performance, safety and durability of the anøde itself and, consequently, full Li-ion cells. In addition, there are challenges with transferring insights from atomistic modelling in a scalable form to models on different length and time scales, while maintaining physical integrity. These outstanding challenges are:

- The role of metastable phases in the kinetics of staging behaviour. New theoretical frameworks should be developed to understand the connectivity between different phases and the effect of this on the path dependency of measurable behaviour like the OCV. These distinct pathways also have implications for mechanical degradation and fracture. A promising approach in this direction is the semi-grand canonical framework developed by Van der Ven et al., Van der Ven et al. describing layered transitions in cathodes $^{\frac{9|231| 278 \mid 279}{1}}$ that could also be applicable to graphite anodes and other candidate materials like silicides.

- The role of the configurational, vibrational and electronic entropy of lithium insertion. Longer length scales, i.e. continuum models, still assume that the entropy follows an ideal solid solution behaviour. The importance of configurational entropy to the phase transitions of lithium in graphite was highlighted in previous sections. ${ }^{\text {71/91|225] }}$ One promising extension would be to use the results from MC calculations to parameterise a phase field model, such as those developed by Bazant, ${ }^{95}$ Guo et al. ${ }^{96}$ and Bai et al., ${ }^{97}$ with a more realistic Hamiltonian and thus include entropy effects in a rigorous way.

- Regarding dynamics, kMC approaches with an empirical Hamiltonian show promise, 102,104|220 but are limited by the length and time scale of the properties that can currently be modelled. A possible solution would be to develop an effective cluster interaction Hamiltonian linking with a linear scaling DFT code, such as ONETEP. Parellelisation 

of the kMC calculations could be achieved by exploiting recently developed graphical processing unit (GPU) architectures.

Superior models of surface and interface effects are needed. This includes development of a physically rigorous version of the Butler-Volmer equation, which is valid for electron transfer but is conventionally assumed to be valid too for ionic transfer in Li-ion batteries. The current models of the interface are too simplistic or represent an ideal situation instead of dealing with the complex reality of the SEI. A systematic coarse-graining approach involving multi-length- and multi-time-scale physics can help in understanding the complex nature of the SEI and its influence on performance of Li-ion batteries. Controlling and improving the properties of SEI is crucial to improve the overall rate capability of Li-ion batteries, as that interface is the bottleneck for Li-ion diffusion.

Regarding graphite, atomistic modelling can be used to predict systematic modifications

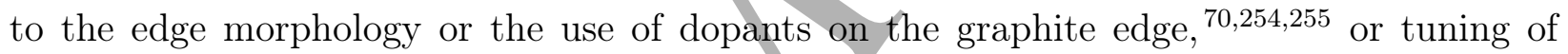
the interlayer carbon spacing 214 to enable systematic tuning of the rate performance. This approach has the potential to lead to more robust interfaces and strategies to tune the anode voltage and dynamics, thus tuning nucleation barriers and mitigating the risk of lithium plating. ${ }^{258}$ In this regard, it should be pointed out that decoupling the rate performance of different graphite edges is still a great challenge from experiment and therefore this finding represents a success for atomistic modelling.

We highlight that there are still outstanding challenges regarding modelling metastable behaviour, volume expansion and degradation in solid solution materials such as silicides. So far, high-throughput atomistic modelling techniques have provided a predictive tool to suggest anode materials that are promising for more extensive experimental characterisation. However, composite materials such as $\mathrm{C} / \mathrm{Si}$ and $\mathrm{C} / \mathrm{SiO}_{x}$, which are increasingly being used in commercial anodes, are presently challenging to model on the atomistic scale. In this regard, an extension to mesoscale modelling, such as a volume averaged approach as suggested by Franco et al., could be a promising way to model challenging materials such as composites, 
1470

${ }_{1471} 4$ Electrolytes

\subsection{Introduction}

1473

1474

in which each component experiences different degrees of volume expansion. 233

Electrolytes are a medium for the transport of charged ionic species, i.e. $\mathrm{Li}^{+}$, between the

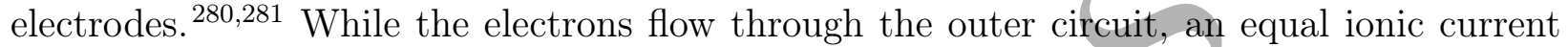
flows through the electrolyte to balance the charge. Electrolytes can be categorised into two groups: liquid and solid, both of which have their benefits and drawbacks. Liquid electrolytes are currently used in commercial lithium-ion batteries (LiBs) and offer high conductivities, but have safety concerns. $\frac{282+284}{2}$ Solid electrolytes are a safer alternative that are approaching commercialisation and can potentially reach higher energy densities. ${ }^{285}$ There are several key aspects to the design of either liquid or solid electrolytes in LiBs: their electrochemical

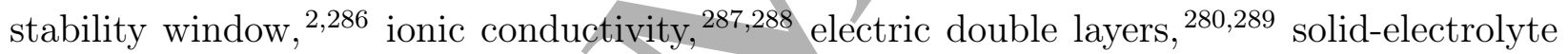
interphase (SEI), $290 \mid 291$ and safety, which are all discussed in the following sections. $\frac{292 \mid 293}{2}$

Electrochemical stability window An electrolyte can be safely used within its electrochemical stability window, which defines the voltage range outside of which it can be oxidised or reduced. ${ }^{2}$ The electrochemical stability window is schematically depicted in Figure 20, showing the electronic energy levels in the electrodes and electrolyte of a battery cell. If the anode electrochemical potential, $\mu_{A}$, is above the lowest unoccupied molecular orbital (LUMO) of the electrolyte, the electrolyte will be reduced. Conversely, if the cathode electrochemical potential, $\mu_{C}$, is below the highest occupied MO (HOMO) of the electrolyte, the electrolyte will be oxidised. Therefore, the electrochemical potentials, $\mu_{A}$ and $\mu_{C}$, should lie within the energy gap, $E_{g}$, between the LUMO and the HOMO of the electrolyte, constraining the open circuit voltage (OCV), $V_{\text {oc }}$, of a battery cell, such that:2

$$
e V_{\mathrm{oc}}=\mu_{A}-\mu_{C} \leq E_{g}
$$


where $e$ is the elementary charge, i.e. the magnitude of the charge on an electron.
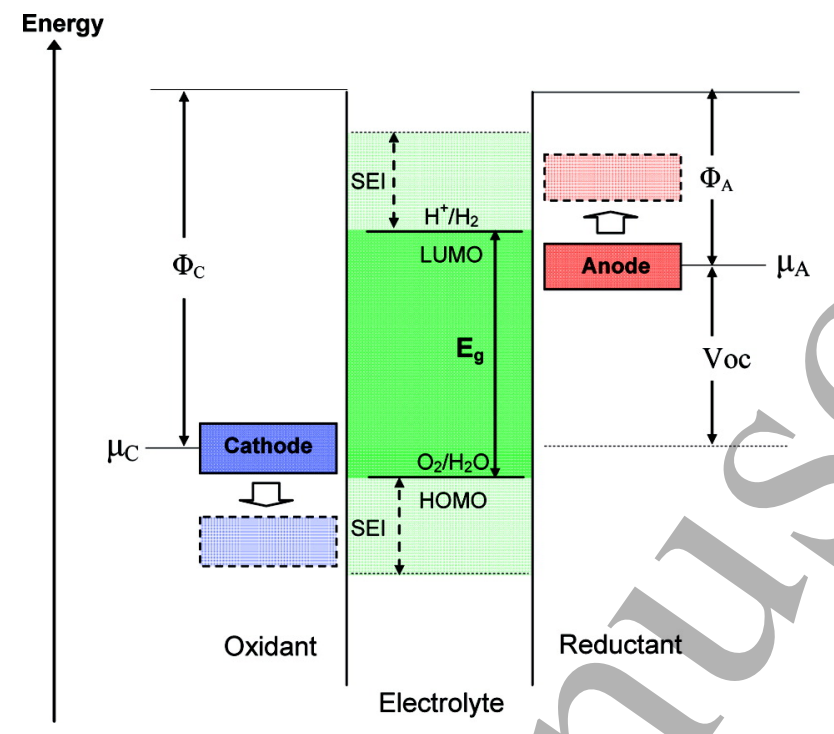

Figure 20: Schematic open circuit energy diagram of an aqueous electrolyte. $\Phi_{A}$ and $\Phi_{C}$ are the anode and cathode work functions. $E_{g}$ is the electrochemical stability window of the electrolyte. If $\mu_{A}>$ lowest unoccupied molecular orbital (LUMO) and /or $\mu_{C}<$ highest occupied MO (HOMO), the electrolyte would be thermodynamically unstable and its usage would require kinetic stability through the formation of a solid-electrolyte interphase (SEI) layer. Reprinted with permission from Ref. 2. Copyright 2010 American Chemical Society.

The energy gap, $E_{g}$, for an aqueous electrolyte is $\sim 1.3 \mathrm{eV}$, severely limiting the $\mathrm{OCV}$, $V_{o c}$. In order to obtain a higher $\mathrm{OCV}$, non-aqueous electrolytes with larger $E_{g}$ have been used in LiBs. ${ }^{[2292]}$ A good summary of electrochemical stability windows of different classes of nonaqueous electrolytes including (organic and inorganic) liquids, solids, ionic liquids, polymers and their combinations is presented by Goodenough and Kim. ${ }^{2}$ Commonly used organic liquid electrolytes, such as $1 \mathrm{M} \mathrm{LiPF}_{6}$ in 1:1 ethylene carbonate (EC) : dimethyl carbonate (DMC), have stability windows between $\sim 1.3-5.0 \mathrm{~V}$, while ionic liquids have stability window between $\sim 1.0-5.3 \mathrm{~V}$. A desirable property of solid electrolytes is their larger electrochemical stability window $(\sim 0.0-8.0 \mathrm{~V})$, compared to liquid electrolytes, ${ }^{[2}$ allowing them to operate within a larger voltage window and thus increase the energy density of the battery.

Ionic conductivity High ionic conductivity $\left(>10^{-4} \mathrm{~S} \mathrm{~cm}^{2}\right)$ in the electrolyte (liquid 1505 or solid) and across the electrode-electrolyte interphase enables a high rate-capability of the 1506 overall Li-ion battery. ${ }^{21287 / 288}$ Generally, the ionic conductivity of liquid electrolytes is higher 

than that of solid electrolytes. However, new classes of solid materials have been found with ionic conductivity surpassing that of liquids (cf. section 4.3), known as superionic conductors. The ionic conductivity of commonly used liquid electrolytes is several orders of magnitude higher than that in the bulk of electrodes and the electrode-electrolyte interphase. ${ }^{287}$

Electric double layer During the charging of an electrode in contact with a liquid electrolyte, excess charge develops at the electrode surfaces. This triggers the rearrangement of electrolyte ions in the electrolyte solution, such that counter-electrolyte charges accumulate near the electrode-electrolyte interface, forming an interfacial charge density perturbation, to achieve local electroneutrality at the interface. In the classical system of dilute electrolytes, electroneutrality is achieved by the formation of a monotonically decaying 'double layer'.280 The double layers in solid electrolytes cannot be directly observed experimentally, so modelling can be used to rationalise their effects. Several models of the electric double layer in electrochemistry exist, such as Helmholtz, Gouy-Chapman, and Gouy-Chapman-Stern. 281 Early models were limited in sophistication: the Helmholtz double layer model suggested charge screening by a plane of counter-charged electrolyte ions near the electrode surface, resembling a capacitor. In contrast, the Gouy-Chapman model screens charge via a diffuse layer of electrolyte ions, decaying monotonically to their bulk concentration value, where the electric potential will fall to zero. The Gouy-Chapman-Stern model accounted for discrepancies encountered by including both a Helmholtz layer of counter charge, as well as a diffuse layer of electrolyte ions, as shown schematically in figure 21(a). These continuum models of electrolyte ions are also being integrated with quantum mechanical methods, such as Density Functional Theory (DFF) (c.f. section 2.2.1). Bhandari et al. recently implemented such a hybrid quantum-continuum model to achieve electroneutrality in simulations of charged electrochemical interfaces, based on a modified Poisson-Boltzmann equation (PBE). [6] At the interface between solid electrolytes and electrodes, a similar decay in charge is observed. However, in this case, the charge carrier is the charge vacancy. Maier discuss the theory of this decay in detail ${ }^{294}$ and new continuum models continue to be developed for 
solid electrolytes. ${ }^{295} \sqrt{298} \mid$ Swift et al. present a model for formation of the double layer in solidsolid electrochemical interfaces, based on the Poisson-Fermi-Dirac equation. The resulting space charge layer of point defects in a solid electrolyte material is shown schematically in figure 21(b). However, this study only accounts for the effect of correlations between ions by limiting the concentration of defects in the interfacial layer to be below a certain value.

At higher concentrations, screening of electrodes changes markedly in liquids, with a new regime emerging when the Debye screening length is of roughly equal value to the ionic diameter. In this regime, charge is screened by means of exponentially damped oscillations of counter-ions and co-ions, in an ordered interfacial structure known as overscreening, 299 a structure that has previously been observed experimentally for liquids. $\frac{300+303}{303}$ In 2021 , Dean et al. became the first to propose the existence of a similar oscillatory decay at solid electrolyte grain boundaries. $\stackrel{304}{\underline{304}}$

a

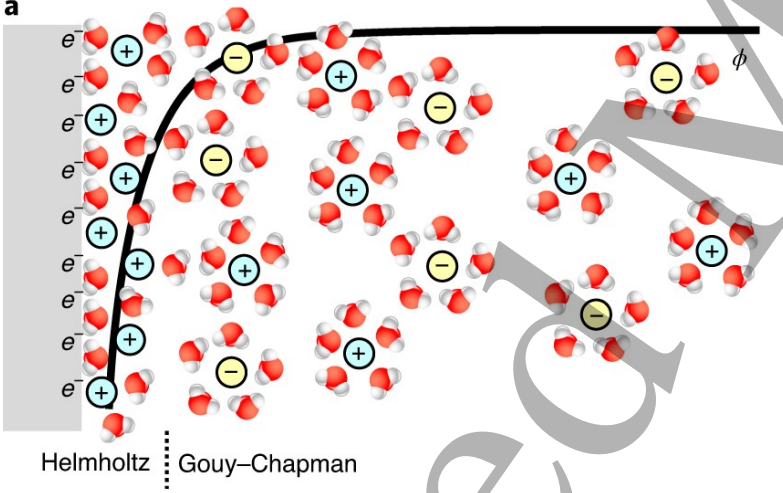

b

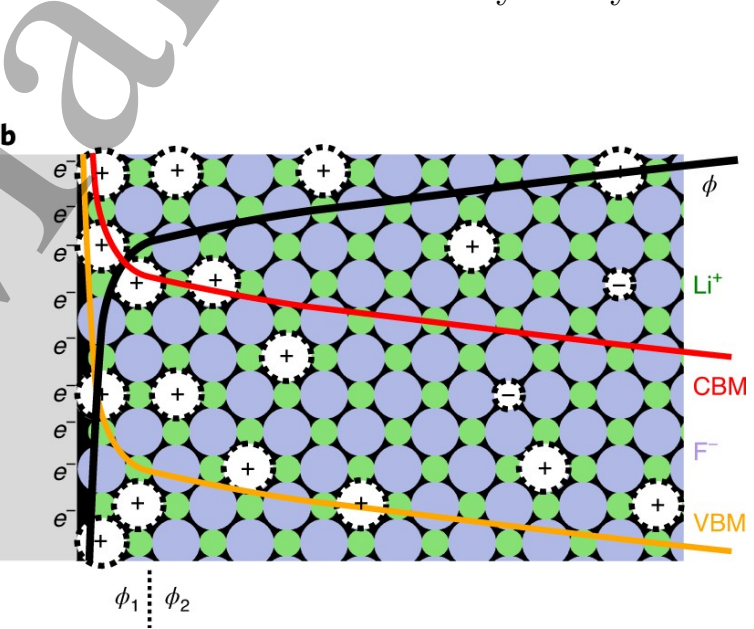

Figure 21: Schematic comparing the double layer formed at the solid-liquid and solid-solid electrochemical interfaces. (a) For the solid-liquid interface, excess electrons on the electrode are balanced by increased density of solvated positive ions in the liquid electrolyte. $\phi$ is the electrostatic potential and is mediated by the Helmholtz layer, followed by exponential decay in the diffuse layer (described by Gouy-Chapman theory). (b) For the solid-solid interface, excess electrons on the electrode are balanced by increased density of positive point defects in the solid electrolyte. Electronic band bending occurs in the solid electrolyte. $\phi_{1}$ and $\phi_{2}$ are the electrostatic potentials next to and further from the interface. Electronic bandbending is shown via the valence-band maximum (VBM), also known as the highest occupied molecular orbital (HOMO), and conduction-band minimum (CBM), also known as the lowest unoccupied molecular orbital (LUMO). Reproduced with permission from Springer Nature: Ref. 289, Copyright 2021.

Solid-electrolyte interphase The "interface" described above is basically a two-dimensional 
surface between the electrode and electrolyte. In LiBs, the electrolyte reacts irreversibly and decomposes on the electrode surfaces, leading to the formation of a distinct phase, several nanometres thick, between the electrode and the electrolyte, known as the-SEI. ${ }^{290}$ The ability to form a stable interphase, which is both ionically conducting and electronically insulating, is an important criterion for the selection of an electrolyte material. The electron insulating property of the SEI is important, to stop further decomposition of the electrolyte on the electrode. ${ }^{21292}$ High ionic conductivity through the SEI is important, otherwise this can form a bottleneck for the overall rate capability of LiBs. $\stackrel{16 \mid 293}{ }$ While the SEI was originally discovered in liquid electrolytes, its rate-limiting behaviour is now also observed in all-solid-state batteries (ASSBs). 291

The two major classes of electrolyte materials, solid and liquid electrolytes, are discussed separately. We focus on the atomistic modelling of different types of liquid and solid electrolytes and their battery related properties. For the liquid electrolyte section, this includes the bulk structure, diffusion properties, solvation/energies, and activity coefficients of different solvents. For the solid electrolyte section, there is a particular emphasis on the ion transport mechanisms, material stability, and the electrode-electrolyte interfaces. Finally, we discuss the individual challenges and outlook for future atomistic modelling of both liquid and solid electrolytes.

\subsection{Liquid Electrolytes}

\subsubsection{Introduction to liquid electrolyte materials}

The most widely used liquid electrolyte in Li-ion batteries is $\mathrm{LiPF}_{6}$ in a solvent, which is typically a mix of two or more solvents, for example EC, DMC, propylene carbonate (PC), or ethyl methyl carbonate (EMC), in order to achieve the competing objectives of dissolution of a high concentration of salt, low viscosity, and high dielectric constant at

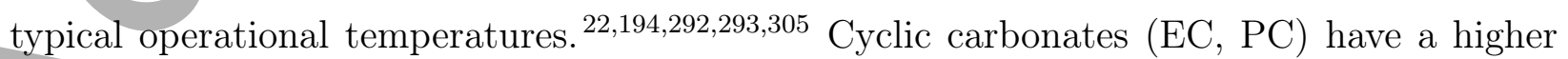
dielectric constant but also high viscosity, while "linear" carbonates (DMC, EMC) have 
low viscosity but also a low dielectric constant. For that reason, mixtures of solvents are often used to optimise performance in a specific application. ${ }^{22|194| 306}$ However, in the last two decades there has been continued innovation in electrolyte mixtures, including ionic liquids $\stackrel{307}{307}$ and salt in water-based systems. $\frac{308}{2}$ This section will touch on both traditional and emergent electrolyte solvents.

\subsubsection{An introduction to modelling liquid electrolytes}

The modelling of liquid electrolytes for conventional batteries is a broad and diverse field. Over the past 20-30 years, atomistic modelling has helped to shape the fundamental physics of liquids, determining a new physical basis and validating decades-old pen and paper theories of concentrated electrolytes. $\frac{309}{312}$ Here, we focus on the development of liquid electrolyte models and the considerations needed when modelling these materials, before moving on to their applications in measuring different properties.

Atomistic modelling of liquid electrolytes can/be broadly separated into ab initio and classical (potentials-based) Molecular Dynamics (MD) modelling (c.f. section 2.1.6). These are complementary techniques which can be used to aid each other. For example, ab initio calculations are able to provide information on the electron distribution, required for parameterising the non-bonded components of force fields used in classical MD. Classical MD can also be used to provide the starting conditions for DFT calculations. Ab initio and classical methods can also be combined in quantum mechanics/molecular mechanics (QM/MM) studies, where the larger system is treated classically with a smaller sub-region being modelled using ab initio methods. For example, a study by Fujie et al. used the "Red Moon" method to investigate the formation of the SEI at the metallic electrode. $\underline{313}$

In this section, we first discuss the separate design and use of $a b$ initio and classical MD methods, followed by their application to determine properties in the bulk liquid electrolyte. Finally, we discuss the application of atomistic methods to SEI investigations, from the 1598 perspective of the liquid electrolyte (complementary to the solid-focused SEI discussion given 
1599

1600

1601

1602

1603

1604

1605

1606

1607

1608

1609

1610

1611

1612

1613

1614

1615

1616

1617

1618

1619

1620

1621

1622

1623

\subsubsection{Ab initio modelling of liquid electrolytes}

Ab initio calculations on liquid electrolytes provide critical information that has been used to explain their behaviour in experimental applications. For many years, DFT calculations (c.f. section 2.1.1) have been used to provide information on the electrochemical stability of solvents. ${ }^{[14}$ Modelling the electrochemical stability allows more complex effects to be decoupled, which is not possible through experimental techniques, and these models have aided the understanding of the functional form of the LUMO and HOMO, opening routes to raise the stability window by design. Computational models were further developed in when Ong et al. used a combined MD and DFT approach to model the electrochemical window of several ionic liquids with a higher degree of accuracy than previously thodology has since been widely used in studying the stability of various hich was later extended to model fluctuations in the coordination shells, $\frac{317}{2}$ and copper ${ }^{318}$ ions and the redox of molecular species. ${ }^{[19}$ However, the applicability ef any such method is somewhat dependent on the solvent. This point was made clear by Lynden-Bell on the subject of the difficulties of applying Marcus theory to ionic liquids, where long range electrostatic interactions may become important. ${ }^{320}$ This type of modelling is important, as single atom events cannot easily be viewed in isolation experimentally, with a temporally and spatially averaged perspective of the system being obtained using most experimental probes. In explicit atomistic simulations, behaviour can be observed at an atomic scale, as shown in further detail in section 4.2 .5 .

${ }^{1624}$ liquid electrolytes, using the PBE-generalised gradient approximation (PBE-GGA) exchange- 
correlation functional to calculate the statistical and dynamic properties. $\frac{\sqrt[321]{121}}{\text { They performed }}$ simulations of $\mathrm{LiPF}_{6}$ at $310 \mathrm{~K}$ and $400 \mathrm{~K}$ in EC and PC at densities comparable with typical experimental compositions. They observed a spontaneous decomposition of $\mathrm{LiPF}_{6}$ into $\mathrm{Li}^{+}$ and $\mathrm{PF}_{6}^{-}$and a coordination number of 4 for solvated $\mathrm{Li}^{+}$, similar to experimental observations. The plots of the radial distribution function (RDF) of Li-ion with the carbonyl oxygen of EC and PC are shown in Figure 22. The Li-O (carbonyl) near-neighbour distance in PC is found to be $\sim 1.94 \AA$ at $310 \mathrm{~K}$ and $\sim 1.90 \AA$ at $400 \mathrm{~K}$, quite close to the experimentally measured distance of $\sim 2.04 \AA$ by time of flight neutron scattering experiments. ${ }^{322}$ The Li-O (carbonyl) peak for $\mathrm{EC}$ is $\sim 1.92 \AA$ at $310 \mathrm{~K}$ and $\sim 1.90 \AA$ at $400 \mathrm{~K}$, which is quite close to that for PC. Comparatively, a classical MD simulation predicted a Li-O (carbonyl) peak at $\sim 1.70 \AA .323$ The $\mathrm{Li}-\mathrm{O}=\mathrm{C}$ bond angle distribution is shown $1 \mathrm{n}$ the inset of Figure 22. The center of the distribution for $\mathrm{PC}$ is at $140^{\circ}$ which is in agreement with the experimentally

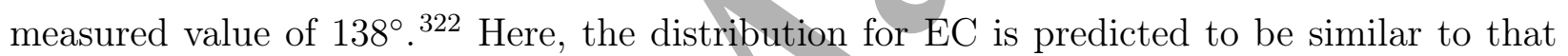
for PC. Calculations using classical MD simulation also predict EC and PC to have similar distributions, though at a much higher $\mathrm{Li}-\mathrm{O}=\mathrm{C}$ angle $\sim 160^{\circ}$ for both solvents..$^{.323]}$

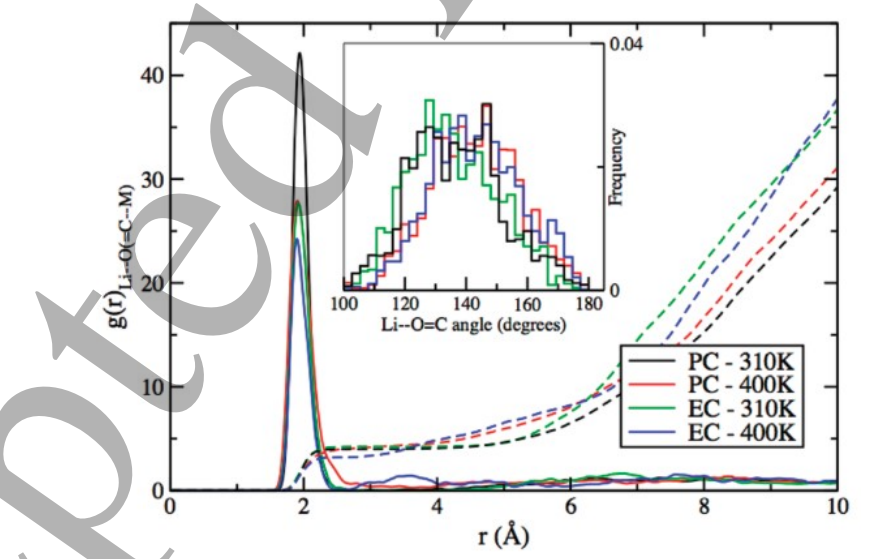

Figure 22: Partial radial distribution function of Li-ion with the carbonyl oxygen of EC and PC along with the partial-density weighted integral (dashed lines) which equals the Li-ion coordination number. In both electrolytes, the Li-O (carbonyl) distance is $\sim 2 \AA$ and the first-solvation shell of Li-ion has $4 \mathrm{EC}$ or PC molecules, consistent with the experiments. The inset shows the histogram of the $\mathrm{Li}-\mathrm{O}=\mathrm{C}$ angle. Reprinted with permission from Ref. 321. Copyright 2011 American Chemical Society.

Perhaps the most enticing possibility regarding ab initio methods at interfaces is to study 

the liquid-electrode interfacial behavior. The physics of such a study are, however, complex and therefore trade-offs in functional choice and solvent model may need to be made, in order to make calculations feasible. Lespes and Filhol used an implicit solvent model to study the interfacial electrochemistry of lithium EC solutions. $\frac{324}{32}$

While AIMD is free from the effects of arbitrary parameters and is highly/accurate, a major limitation of this approach is the high computational cost, restricting the reachable time- and length-scales to just tens of pico-seconds and between hundreds (conventional DFT) and thousands of atoms (linear-scaling DFT approaches, c.f. section 2.1.2, resulting in inaccuracies and irregularities in the calculations.

When considering the impacts of small length scales, the critical issue is the introduction of spurious long- to medium-range correlations of atoms and molecules. As liquids do not exhibit long-range order, the presence of periodic images that are located at exactly a cell's width in all directions introduces an unphysical correlation. This is observed in the modelling of systematically disordered solids in smaller cells. ${ }^{325}$ For example, Zhao et al. recently revealed that there is a distribution of different, low-symmetry, local motifs in cubic halide perovskites, such as tilting and rotations, which are only observed if you allow for a larger-than-minimal cell size. ${ }^{326}$ Beyond truncating the RDF to a shorter distance than is optimal (i.e. half the shortest distance between periodic images), this effect will also introduce (normally small) inaccuracies in thermodynamic and dynamic quantities. $\frac{85 \mid 327+329}{32}$ These inaccuracies are of a particular concern in liquid electrolytes, as the electrostatic interactions between ions gives rise to longer range interactions, even when the Debye length is far smaller than the system size.

The short time scales of $a b$ initio simulations can, particularly for more viscous liquids, lead to highly non-ergodic (fully-sampled) simulations. When snapshots throughout the whole trajectory are highly correlated, 331 this can lead to problems for both dynamic and equilibrium studies.

Often, neither time correlation nor finite size have a significant detrimental effect on 

the reproducibility of experimental results in ab initio studies. However, in specific studies where they need to be avoided, or where a quantum description of a liquid electrolyte provides no significant advantage over a classical description, it is beneficial to turn towards far less computationally expensive potentials-based simulations, allowing larger and longer simulations.

\subsubsection{Classical modelling of liquid electrolytes}

Classical simulation of liquid electrolytes includes classical force field-based MD (c.f. section 2.1.6) and the related field of classical Monte Carlo (MC) (c.f. section 2.1.5). Classical MD, also known in solid-state communities as potentials-based MD, is a broad field which uses many different types of force fields for different studies. The development of force fields for ionic solids is described in section 2.2.2, whereas here we evaluate the force fields used for liquid electrolytes and the considerations for developing them. Historically, force fields for different electrolyte systems have developed at similar paces. Here, we use the example of the development of force fields for ionic liquids.

Electrolyte solvents, from/water to molecular solvents and ionic liquids, pose a challenge that is not normally present in the solid-state, specifically the need to model covalent bonding. This is achieved by splitting the potential acting on each atom into bonding and nonbonding contributions. The non-bonding component accounts for the effects of electrostatics, dispersion, and degeneracy pressure; and the bonding component accounts for the effects of covalent bonding. In classical modelling of liquid electrolytes, we are mainly interested in the behaviour within the electrolyte's electrochemical stability window (c.f. section 4.1). Therefore, the vast majority of classical studies model bonds with unbreakable, harmonic

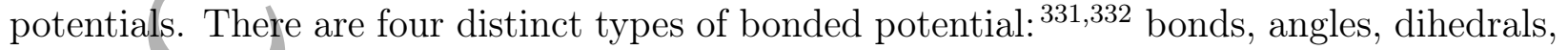
and improper dihedrals. These can be traced back to the parameterisation of force fields, such as OPLSA-AA, $\stackrel{333[334}{3}$ and are often parameterised from spectroscopic force constants. 
their discussion is beyond the scope of this review. Atoms which are subject to a bonded potential are often wholly, or partially, excluded from non-bonded interactions, though in large molecules, non-bonded intramolecular interactions are important. Alternatively, bonds can be kept rigid using a constraint algorithm.

When developing force fields, generally, it is the non-bonded force field components, in particular the partial charges on atoms, which are more frequently varied. A common model for liquid electrolytes is the OPLS-AA force field. ${ }^{334}$ This is a Lennard-Jones potentialbased force field with an additional coulombic term. 338 Further developments can be made from this base force field, such as the CP\&P force field, 333342,344 describing a wide range of ionic liquid cations and anions. Some non-bonded parameters, particularly charges, were varied from OPLS-AA. The charges on the individual molecules are obtained from DFT calculations, in this case by use of the charge mapping algorithm $\mathrm{CHelpG}^{333}$ (though other algorithms may also be used. $\underline{345+347}$ )

Electrostatic interactions are important when modelling charged electrolytes, as are the effects of polarisability. Often it is advantageous in a non-polarisable force field to scale the

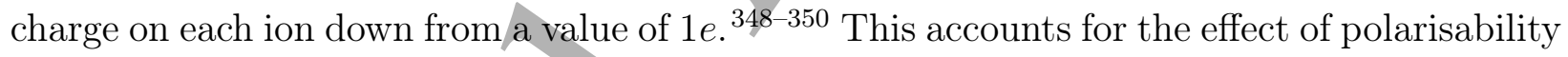
on the strength of electrostatic interactions between ions, which is particularly important for transport properties. However, other force fields have been defined to account directly for polarisability. ${ }^{349}$ As described in section 2.2 .2 , polarisability can be introduced to a force field by the employment of Drude Oscillators (core shell model). 332 348/349 This approach is computationally cheap and is core to the polarisable ionic liquid force field developed from CL\&P by Schröder ${ }^{348}$ A more advanced representation of polarisability can be provided by intrinsically polarisable force fields, normally based on the Fumi-Tosi potential. $\stackrel{351}{\square}$ This method has been used for molten salts, $\stackrel{352}{ }$ ionic liquids,, 34953 and lithium salts in molecular solvents. $\frac{354-356}{3}$ This provides the best description of polarisability in a classical force field, however, there is an associated higher computational cost, and a particular code, such as 1720 metalwalls, $\frac{357}{3}$ ) is often required to implement it. 
1721

1722

1723

1724

1725

1726

1727

1728

1729

1730

1731

1732

1733

1734

1735

1736

1737

1738

1739

1740

1741

1742

1743

1744

1745

1746

The development of force fields for metal cations has seen an equal level of discussion and interest. These cations can be slightly easier to model, owing to their relative nonpolarisability. ${ }^{34913588599}$ They are frequently modeled as Lennard-Jones spheres to match the potential in the prevailing solvent models (SPC and OPLS-AA). For alkali and alkali earth metal cations, a wide range of values of $\sigma$ (excluded volume) and $\epsilon$ (interaction strength) can be used, as the basic energetics associated with one of these force fields can be recovered for many pairs of sigma and epsilon values. The choice of which pair of parameters to use is normally driven by which property requires the highest degree of accuracy for the targeted

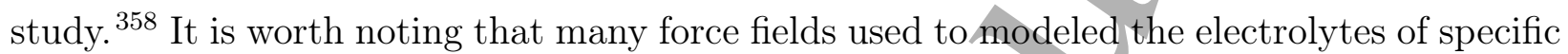
interest to us here, were parameterised for aqueous solutions. $\stackrel{358}{3}$

\subsubsection{Bulk Structure and Landscaping}

For structural analysis of liquid electrolytes, analysis of the RDF is the mostly widely used approach. Modelling of structural properties in this capacity provide more information-rich data compared to scattering, especially in complex systems, and is less labour intensive. RDFs can be converted to structure factors by a simple Fourier transform into reciprocal space, allowing for easy comparison with experimental structure factors, ${ }^{350 \mid 360+362}$ subject to re-scaling for the specific intensities associated with different atoms. This method has been used frequently for a broad array of electrolytes and has seen particular utility for ionic liquids, where the large, inhomogenous ion surface can lead to complex patterns, for which MD can provide explanation. Modelling of this sort of behaviour has been performed for

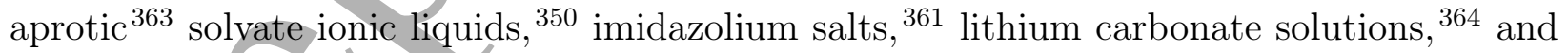
highly concentrated aqueous solvents. $\frac{360}{.}$

The RDF is closely related to potential of mean force acting on a particle, however, the physical relevance of RDFs goes further than this. The mean force describes the changing potential landscape acting between particles as they approach one another $\cdot \stackrel{331}{3 n f o r m a t i o n}$ on the potential of mean force is exceptionally challenging to obtain experimentally, which is 
why modelling techniques are frequently used in colloidal systems, as outlined below, where this information is also of interest. $330[365+370$

The form and gradient of the decay of the RDF towards an asmyptote is crucial when studying the charge screening properties of an electrolyte. These charge screening properties have a particularly prominent effect on electrolyte behaviour when confined ${ }^{299}$, for instance, in a nanoporous electrode. ${ }^{371}$ Surface force experiments ${ }^{365}$ and fluorescence ${ }^{\sqrt{366}}$ based methods do provide an indirect experimental probe of charge screening. However the form of the decay in the correlations between charges, which can be either damped oscillatory or monotonic, cannot be observed, and both methods are restrictive in the systems that can be studied. Atomistic studies of this decay are able to resolve the form of this decay with a great deal of precision, allowing direct calculation of many body correlations. $\frac{330 / 367 / 368}{\text { This provides }}$ validation to integral equation theories, which can describe confined electrolytes with greater accuracy. $\frac{369 \mid 370}{3}$

As well as being generated from an RDF, the potential of mean force can be obtained by direct calculation by use of centre of mass pulling, umbrella sampling,, 332 or running multiple calculations with ions frozen an exact distance apart from one another. When modelling liquid electrolytes, this method is also used to study the approach of ions to an electrode, where the energetics associated with decoordination from the solvent and coordination to the electrode can be modelled. Such information about the energetics of ion approach is not directly accessible experimentally and therefore this allows us to understand the liquid electrode interface more intimately. For instance, in the solvate ionic liquid $[\mathrm{G} 4(\mathrm{Li})]_{+}[\mathrm{TFSI}]_{-}$, a lithium sulfur electrolyte, this sort of analysis gives an understanding of the interplay between dechelation and approach to electrode which would not have been observable experimentally, nor explainable with mean field theory. $\stackrel{372[373}{[n}$ In another example, Sergeev et al. looked at the approach of oxygen and lithium based species towards electrodes. ${ }^{374}$ Here, the authors performed MD simulations of the electrode/electrolyte interfaces of a $\mathrm{Li}-\mathrm{O}_{2}$ cathode 1773 with an experimentally relevant potential in $1 \mathrm{M}$ dimethyl sulfoxide (DMSO) solution of 

$\mathrm{LiPF}_{6}$ salt. They found that oxygen anions are effectively pushed out of the reaction layer, making the second reduction of superoxide anion improbable, indicating the main cause of the electrode surface passivation is the presence of lithium superoxide near the electrode surface. This mechanistic result could not be obtained experimentally due to the number of side reactions; however, the ability of atomistic modelling to simplify the problem allowed for the mechanism to be elucidated. Sergeev et al. proposes a way to suppress the passivation by shifting the equilibrium $\dot{\mathrm{O}}_{2}^{-}+\mathrm{Li}^{+} \rightleftharpoons \mathrm{LiO}_{2}$ to the side of separately solvated ions, for example, by using solvents resulting in lower free energy of the ions. 374

\subsubsection{Li-ion Diffusion}

Diffusion (c.f. section 2.3.3) plays a critical role in the operation of liquid electrolytes through its impact on conductivity. However, in liquid electrolytes its impact goes deeper, as the dielectric constant of liquids consists of both dipolar and ionic contributions. These two contributions can be obtained by analysis of the dipole orientation and current auto-correlation functions using the Einstein-Helfand method. For example, Coles et al. performed this analysis on four liquid electrolytes (three in aqueous solvent and one in a common organic solvent mixture): aqueous solutions of $\mathrm{LiCl}, \mathrm{NaI}$, and lithium bistriflimide (LiTFSI), as well as the same LiTFSI salt solvated in an equimolar mixture of dimethoxyethane (DME) and 1,3-dioxolane (DOL) 330 Here, it was shown that for polar solvents, the dipolar contribution is nearly always dominant, with the current making a small corrective contribution which could feasibly be neglected (particularly for more dilute systems). For ionic liquids, which contain ionic species that can exhibit a net dipole, such as TFSI, both dipolar and ionic contributions would be observed. The effect of molecular ions having simultaneous charges and dipoles was explored by Schröder, who showed that even more thorough treatment may be required to observed the impacts of their interplay. $\stackrel{[376}{~ T h e s e ~ c o n t r i b u t i n g ~ f a c t o r s ~ c a n n o t ~}$ be easily/feasibly disentangled experimentally. 
placement, according to the Stokes-Einstein relation. For example, Ganesh et al. calculated the mean-squared displacement of solvated Li-ion in EC and PC solvents from AIMD, as shown in Figure 23. For PC, the self-diffusion coefficient is calculated to be $\sim 0.7 \times 10^{-9}$ $\mathrm{m}^{2} \mathrm{~s}^{-1}$ at $310 \mathrm{~K}$ while the experimentally measured value of self-diffusion coefficient at 303 $\mathrm{K}$ is $\sim 0.16 \times 10^{-9} \mathrm{~m}^{2} \mathrm{~s}^{-1} \stackrel{377}{ }$ For EC, it is calculated to be $\sim 1.0 \times 10^{-9} \mathrm{~m}^{2} \mathrm{~s}^{-1}$ at $310 \mathrm{~K}$, while the experimentally measured value of self-diffusion coefficient at $313 \mathrm{~K}$ is $\sim 0.21 \times 10^{-9}$ $\mathrm{m}^{2} \mathrm{~s}^{-1} \cdot 377$ At $400 \mathrm{~K}$, the calculated diffusion coefficient for PC increases to $\sim 3.7 \times 10^{-9}$ $\mathrm{m}^{2} \mathrm{~s}^{-1}$, while it remains the same for EC. It is notable here that the Li-ion diffusion in the electrolyte solution is 4-5 orders of magnitude higher than that in the bulk of electrodes, e.g. in the graphite anode (cf. section 3.2.4).

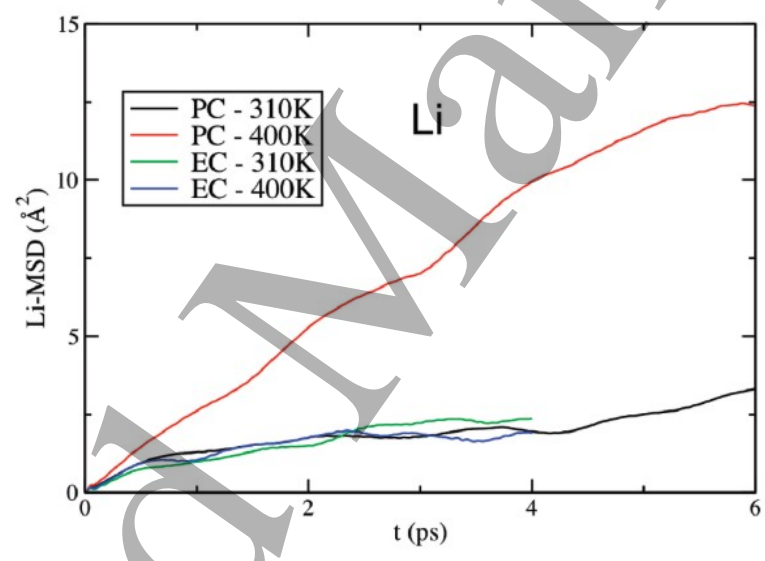

Figure 23: Mean-squared displacement of solvated Li-ion in EC and PC. Reprinted with permission from Ref. 321. Copyright 2011 American Chemical Society.

Investigation of the diffusion of different ions subject to a field gives a sense of the diffusion rate of specific ions and also an idea of exchange rates of solvent molecules. This information can be bbtained using nuclear magnetic resonance (NMR), however, atomistic models can provide more detailed data, as outlined here. For instance, strongly coordinated solvents will have diffusion coefficients closer to the ions they are coordinated to, whereas less strongly coordinated ligands will have diffusion coefficients dissimilar from the coordinating ion. 350354378 380 Examples of this behaviour can be found in the MD studies of Borodin et al., which looked at diffusion in lithium solutions of both the common carbonate ${ }^{\sqrt{354}}$ and 

ethylene glycol oligomer solvents. $\frac{379}{37}$ For the common carbonate, MD predictions of the ion and solvent self-diffusion coefficients and conductivity were in good agreement with experiments, with approximately half of the charge transported by charged ion aggregates

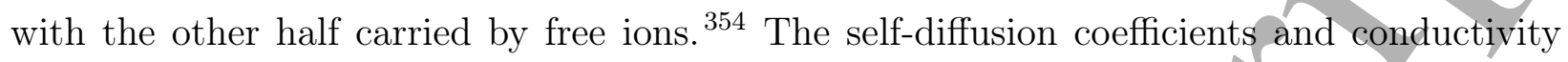
predicted by MD for the ethylene glycol oligomer solvents were also found to be in good agreement with experimental data. $\mathrm{Li}^{+}$transport was found to primarily occur though exchange of $\mathrm{TFSI}^{-}$anions in the first coordination shell. $\frac{379}{\text { The }} 2015$ study of Shimizu et al. investigated a number of different lithium glyme solvate ionic liquids. $\frac{350}{}$ Here, the authors found that although MD was unable to yield quantitative information about the dynamics of the system, it could provide two important pieces of information: the autodiffusion coefficients of glyme molecules in pure glyme are much larger than those of glyme molecules in glyme equimolar mixtures at the same temperature; the decrease in the glyme diffusion coefficients is more pronounced in the $\mathrm{Li}\left[\mathrm{Ntf}_{2}\right]+$ glyme system than in the $\mathrm{Li}\left[\mathrm{NO}_{3}\right]$ + glyme mixture. $\stackrel{350}{30}$ The study of Lesch et al. used MD to investigate lithium salts dissolved in aprotic ionic liquids. 378 The authors found that the exchange of TFSI anions in and out of the first coordination shell/of $\mathrm{Li}^{+}$was faster in $\mathrm{pyr}_{13}$-based systems, compared to emimbased systems, and that the $\mathrm{Li}^{+}$ion transference number was higher. ${ }^{378}$ The atomic scale context provided by atomistic simulation allows for the specific dynamics and atomic scale effects, such as dynamic (de)coordination, which govern diffusion to be observed directly. These behaviours cannot be observed directly in a spatially and globally averaged NMR measurement. 350

In more complex solvents, such as ionic liquids, the nature of the solvent plays a important role too, for instance Borodin and Smith showed the effect of fluorination of ionic liquid cations on diffusion behaviour. .380 This sort of study can be directly compared with pulsed field gradient NMR experiments of battery materials. This was done, for example, when Shimizu et al. studied a LiTFSI-based solvate ionic liquid, which had been proposed as ${ }_{1844}$ a solvent for Lithium Sulfur batteries. $\frac{350}{3}$ The authors found the molecular behaviour of 
solvate ionic liquids to be probed effectively using a combination of MD trajectories and structural/aggregation analysis techniques.

Atomistic simulations are also providing new understanding of the fundamental physics behind non-linear relationships between the electric field and the electrolyte conductiyity, known as Onsager's Wein effect. $\stackrel{381}{{ }^{381}}$ In a recent study of dilute electrolytes and molten salts ${ }^{382}$ Lesnicki et al. performed a direct study of this half century old conundrum, by directly observing the interplay between external fields as well as the correlations between, and clustering of, ions using atomistic modelling and innovative statistical mechanical analysis. This direct observation of liquid structure and dynamics could only be obtained through simulation. Such studies are likely to expand into the study of battery electrolytes in the coming years where this sort of behaviour has a direct effect on conductivity and function. In the above examples of diffusion studies, ion pairing plays a contributing role; in general, any phenomenon originating from ion paring can benefit from investigation by atomistic simulation.

Shimizu et al.'s work highlights a key advantage of molecular simulation. While the authors utilised both scattering and NMR based experimental probes, neither could provide the same unambiguous detail of the nature and dynamics of ion pairing, on the single atom scale in the liquid, that can be obtained from simulation. $\frac{350}{}$ More recently, the richness of simulated data sets has allowed for the analysis of liquid structures with deep learning based approaches, providing a more complete picture of electrolytes, their disorder, and complexity. $\underline{383}$

\subsubsection{Solvation Energies}

Solvation energies in electrolytes have been widely studied and, though research focus has been on aqueous solvation of biomolecules, these techniques can also be used to look at solvation of metal ions with organic solvents. Dependent on the exact thermodynamics 
1871 1872

Skarmoutsos et al. combined DFT and MD methods to look at the solvation structures of lithium salts in ternary mixtures of different carbonate solvents and showed that different solvents were found to dominate at different distances from a central lithium cation, observing a particular preference for solvation of lithium by DMC ions over PC and EC, as shown in Figure 24. Takeuchi et al. looked even deeper at the energetics behind the direct contact between cations and anions in solution. $\frac{385}{35}$ The relative stabilities of the mono-, bi-, and tridentate coordination structures were assessed with and without solvent, where water, PC, and DMC were found to favour the ion pair (CIP)-solvent contact. Vacant sites of $\mathrm{Li}^{+}$cation in CIP are solvated with three carbonyl oxygen atoms of PC and DMC solvent molecules, and the solvation is stronger for the monodentate CIP than for the multidentate. ${ }^{385}$ Such detailed analysis is not possible to resolve through experimental techniques.

These are just a few notable studies on solvation energies in liquid electrolytes. A compelling theoretical description of solvation is given by Lazaridis. 386

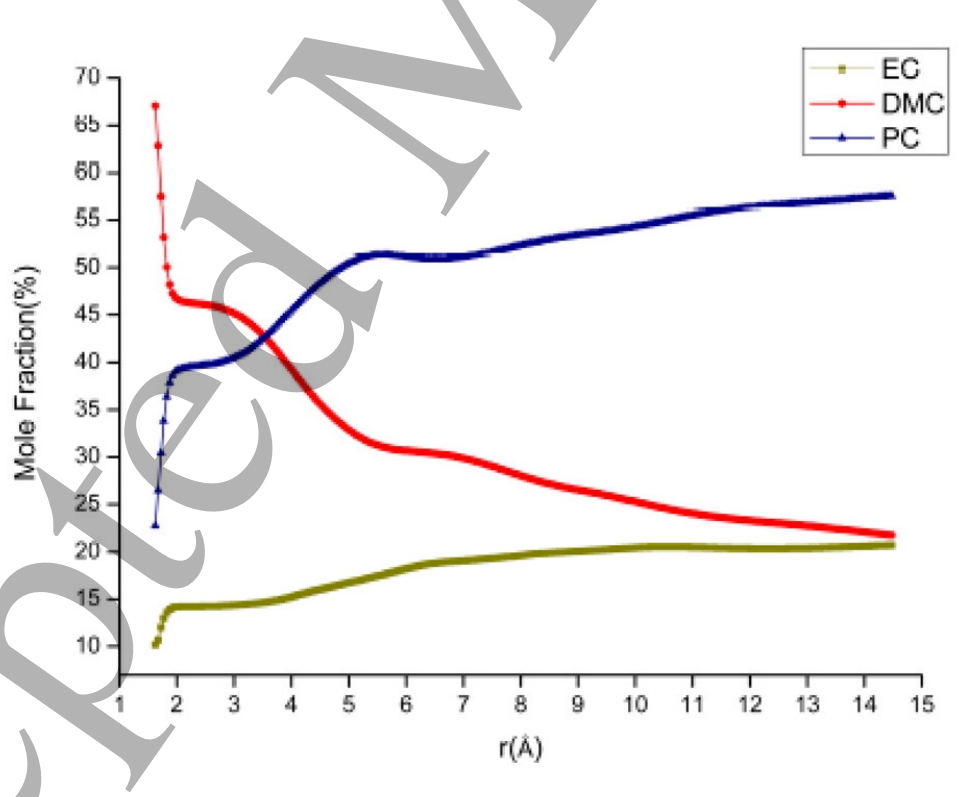

Figure 24: Local mole fractions (\%) of ethylene carbonate, propylene carbonate, and dimethyl carbonate as a function of the distance from the lithium cation in the ternary mixture. Reprinted with permission from Ref. 384. Copyright 2015 American Chemical Society. 
1884

\subsubsection{Activity coefficients of electrolytes}

The activity coefficients describe the deviation of actual electrolytes from an ideal mixture of substances $^{171}$ and can be calculated using DFT + PBE simulations (c.f. section 2.1.1) of solutes in electrolyte solutions, as described in sec 2.3.2. The experimental value of bulk permittivity of EC is $\left(\varepsilon^{\infty}=90.7\right)^{387}$ and its surface tension is $\left(0.0506 \mathrm{~N} \mathrm{~m}^{-1}\right)$. ${ }^{388}$ These values were used by Dziedzic et al. to calculate the activity coefficient of $\mathrm{LiPF}_{6}$ in EC.135 The solvent radius was set to $R_{k}^{\text {solvent }}=10.5 a_{0}$, to approximate the size of an EC molecule, and the isovalue of solute electronic density, $\left(\rho_{\mathrm{e}}^{\lambda}\right)$, is varied to match the experimental activity coefficients. A plot of the computed activity coefficients as a function of the square root of electrolyte concentration is given in Figure 25, along with experimental values from Stewart and Newman. ${ }^{389}$ Here, we see a good agreement for $\rho_{\mathrm{e}}^{\lambda}=0.002 \mathrm{e} / \mathrm{a}_{0}^{3}$. Trends are also plotted from the linearised approximation of $\mathrm{PBE}$, where the solvent radius is reduced to resemble the prediction for point charges from the Debye-Hückel theory. $\frac{390}{3}$ The thermodynamic factor can be obtained from numerically differentiating these curves. This is a novel technique of calculating activity coefficients and thermodynamic factors from hybrid atomistic-continuum methods.

\subsubsection{Interfacial Nanostructure of Electrolytes}

In sections 3.3 and 5.4 , the interfaces between solids and liquids from the perspective of the solid have been discussed. However, the interface from the perspective of the liquid is also of interest. The structure of liquid electrolytes at metallic ${ }^{391}$ and charged dielectric ${ }^{365}$ interfaces will normally extend away from the interfacial region and can be observed prominently for tens of nanometers and, dependent on concentration of the liquid, can either be monotonic or oscillatory, as described in section 4.2. Spectroscopic and surface methods used to study the liquid-solid interface are often indirect and require specific conditions for analysis (e.g. transparency and smoothness) $392 \frac{394}{39}$, which often constrains the interfaces we can study as well as the detail and conclusiveness of the data obtained. Computational modelling provides 


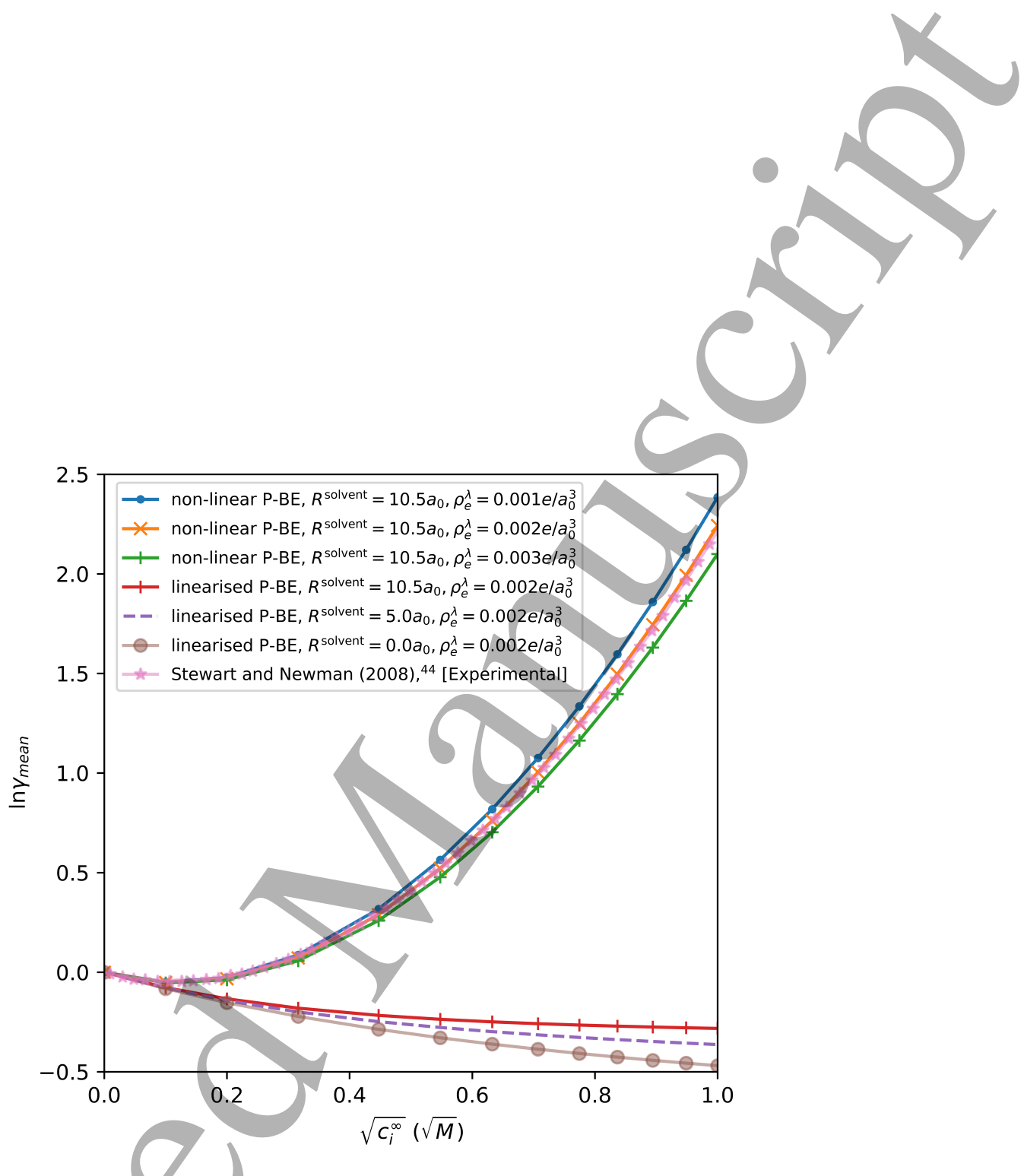

Figure 25: Mean activity coefficients for $\mathrm{LiPF}_{6}$ in ethylene carbonate at $T=308 \mathrm{~K}$ as a function of concentration and for different values of the atomic electronic density isovalue parameter which determines the extent of the accessibility function. Calculations with the linearised approximation to P-BE are also shown. Reprinted with permission from Ref. 135. Copyright 2020 American Chemical Society. 

a route to direct and data rich understanding of the liquid solid interface. This section will highlight some particular areas of study.

Concentrated electrolytes and ionic liquids both adopt the characteristic overscreening structure at charged interfaces, including electrodes. This structure, comprising oseillations

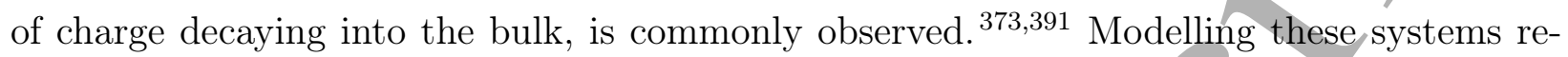
quires an appropriate electrode model. While interesting information can be gained from simulating ions at an electrode with a fixed charge, for example in a high throughput study looking at structural changes with electrode surface charge, ${ }^{[733}$ fixed potential boundary conditions will provide a more accurate description of the capacitance, ${ }^{391395}$ interfacial structuring of a liquid electrolyte, 3733966397 and the decoordination and dechelation dynamics of coordinated ions. ${ }^{398}$ Though we note that, in light of a recent study by Scalfi et al., this field continues to evolve as more nuanced/classical electrode models are employed, such as the Thomas-Fermi based model proposed by Scalfi et al. ${ }^{395}$

A wide variety of different electrolytes have been studied using fixed potential electrolytes, from ionic liquids to concentrated electrolyte. Both nanoporous ${ }^{\frac{\sqrt{371} 139663981399}{3}}$ and nanoscopically rough electrode surfaces, have been heavily used. ${ }^{400}$ A specific example of interest is the work of Borodin and Bedrov, where MD simulations were performed on dilithium ethylene dicarbonate $\left(\mathrm{Li}_{2} \mathrm{EDC}\right)$ and dilithium butylene dicarbonate $\left(\mathrm{Li}_{2} \mathrm{BDC}\right)$, in contact with mixed solvent electrolyte (EC:DMC) doped with $\mathrm{LiPF}_{6} \cdot{ }^{4011}$ In this study, the authors examined the SEI-electrolyte interface and found an increase of EC and PF6- molecules and a decrease of DMC at the interfacial layer next to the SEI surface, compared to bulk electrolyte concentrations. The activation energies for the $\mathrm{Li}^{+}$solvation-desolvation reaction were estimated to be $0.42-0.46 \mathrm{eV}$ for the $\mathrm{Li}_{2} \mathrm{EDC}-$ electrolyte and $\mathrm{Li}_{2} \mathrm{BDC}$-electrolyte interfaces.

While the context provided by these methods is useful, more generally the capacitance curves generated by atomistic studies of fixed potential electrodes have frequently been able to replicate experimental results when mean field theory cannot. For instance, Simoncelli 1936 et al. were able to replicate the experimental behaviour, in particular the double layer ca- 
pacitance, of a nanoporous brine based capacitor, where a variety of mean field models were unsuccessful. ${ }^{402}$ Li et al. were able to show the exact rearrangements of the molecules and ions at a metallic interface which gave rise to the nature of the experimentally and computationally observed relationship between capacitance and voltage of a water in salt electrolyte. $\stackrel{397}{3}$

\subsubsection{Outlook and challenges}

Liquid electrolytes will likely remain the most prominent form of commercialised electrolyte for battery applications in the near future. This is partly due to their monopoly in the market and partly due to their low cost, which will continue to drive popularity. Despite the overwhelming success of commercial liquid electrolytes, there is still room for further performance improvements, with several key issues as limiting factors. Liquid electrolytes are known to be limited by narrow electrochemical windows, solvent toxicity, and material flammability/safety concerns. There are two potential avenues for solving these issues:

- Resolving these limitations within the confines of liquid electrolytes: ionic liquids have a large electrochemical window and high thermal stability, and their conductivities are similar to those of conventional organic solvent solutions. ${ }^{[07}$ However, they are expensive and there are associated safety concerns. ${ }^{[282[283} \mathrm{A}$ liquid electrolyte alternative to this could be in water-in-salt electrolytes. Water-in-salt electrolytes are a novel class of electrolytes, which inverts the conventional idea of a salt being dissolved in a solvent, with a small amount of water being dissolved in a hygroscopic lithium salt to the point where a/liquid is obtained,, 4031404 analogous to the high concentration organic electrolyte solutions described by Yamada et al. ${ }^{306}$ These liquids have the advantage of being comprised solely of a lithium salt and water, which decreases cost and eliminates the toxicity and risk of flammability and thermal runaway traditionally asseciated with organic solvents. The high concentration of salt also leads to a greatly expanded electrochemical window of $3 \mathrm{~V}^{308}$ from the $1.23 \mathrm{~V}$ value for dilute aqueous 
solutions. However, the highly concentrated solutions in these electrolytes lead to re- 
SEI, conducive to ion transmission. ${ }^{16}$ Further details of the formation and function of the SEI at the graphite anode are summarised in section 3.3 .5 .

Liquid electrolytes are complex substances and are therefore difficult to fully capture in atomistic models. In recent years, computational capacity has expanded, allowing more complex models to be studied. Alongside this, new computational methods have been developed under the open source license, allowing research of these materials to become more accessible. .357371401402 Future advances in computational ability, combined with improved experimental studies, provide a framework for high throughput screening of electrolyte materials.

Developments in expanding the achievable time and length scales of AIMD will allow more complex models to be developed. However, it is still implausible that AIMD will be able to simulate whole electrodes/interfacês/battery cells for long enough time and length periods to achieve full ergodicity (statistical convergence). Therefore, methods which can provide long scale simulations are still needed. In particular, the emerging fields of fitting machine learnt potentials for liquid electrolytes,, $13 \sqrt[415]{41}$ and more complex classical models which incorporate polarisability ${ }^{3491357}$ or bond breaking dynamics. ${ }^{4161417}$ This would enable simulations of electron transfer, bond formation, and the effect of ion and solvent polarisability at larger scales and in greater detail.

Atomistic modelling of liquid electrolytes does not necessarily require more computational expense to advance. Exploitation of underused physical methods to model liquid systems at far lower cost has been explored. One such method, classical DFT, has already been applied to model aqueous capacitors ${ }^{369}$ and confined ionic liquids. $\frac{418}{}$ This has the potential to be coupled with electronic DFT (c.f. section 2.1.1) to model electron transfer. $\underline{419}$

It should be emphasised that, for practical use, the interfaces between the liquid electrolyte and the electrodes are the major limiting factors in terms of performance, stability, and safety. Therefore, advancement through electrolyte design is crucial, where the critical obstacles discussed here could be resolved by the use of novel, solvents, salts, or electrolyte 
2016

2017

2018

2019

2020

2021

2022

2023

2024

2025

2026

2027

2028

2029

2030

2031

2032

2033

2034

2035

2036

2037

2038

2039

2040

2041

salts. Several articles discuss the challenges of this topic in greater detail. 420,422

\subsection{Solid Electrolytes}

\subsubsection{Introduction}

Solid electrolytes have attracted considerable attention as an alternative to highly-flammable liquid electrolytes, as they significantly improve device safety and have the potential to improve energy and power densities, while also reducing the cost of synthesis. ${ }^{285 \mid 423}$ An ideal solid electrolyte material should possess high electronic resistance, high ionic conductivity, outstanding thermal stability, strong electrochemical stability, excellent mechanical strength, and reduced interfacial resistance. ${ }^{427 / 428}$ There are three different categories of solid electrolytes used in rechargeable batteries: $\stackrel{426}{(1)}$ inorganic ceramic electrolytes, (2) organic polymer electrolytes, and (3) composite electrolytes.

Solid electrolytes were discovered by Michael Faraday in the early 1830s through research on the conduction properties of heated solid silver sulfide $\left(\mathrm{Ag}_{2} \mathrm{~S}\right)$ and lead fluoride $\left(\mathrm{PbF}_{2}\right) . \underline{429}$ The use of a ceramic-based $\beta$-alumina $\left(\mathrm{Na}_{2} \gamma \cdot 11 \mathrm{Al}_{2} \mathrm{O}_{3}\right)$ in high-temperature sodium-sulfur (Na-S) batteries in the 1960s was considered as a milestone in the development of batteries,

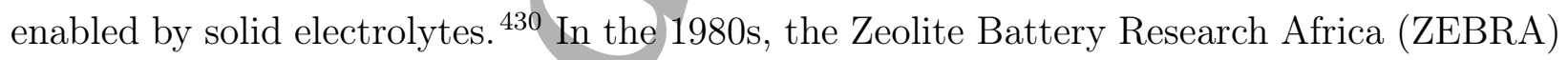
group developed the "ZEBRA" batteries using $\mathrm{Na}_{2} \mathrm{O} \cdot 11 \mathrm{Al}_{2} \mathrm{O}_{3}$ as the solid electrolyte. 431 So far, the Na-S battery has been commercialised in Japan, $\stackrel{432}{ }$ whereas the ZEBRA battery is currently being developed by the General Electric Corporation in the United States. $\stackrel{433}{4}$

In 1990, the Oak Ridge National Laboratory synthesised a lithium phosphorus oxynitride (LiPON) material, $\frac{4341435}{4}$ opening up the use of inorganic solid-state electrolytes in LiBs. Since then, a huge number of inorganic, lithium-ion conductive ceramic materials have been developed, including perovskite-type, $\stackrel{436}{ }$ garnet-type oxides, $\stackrel{437438}{ }$ garnet-type sulfides, $\underline{439}$ lithium super ionic conductor (LISICON), $\stackrel{440}{2}$ sodium super ionic conductor (NASICON)-

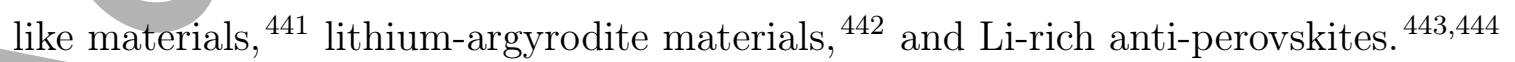

Despite recent advancements in crystalline inorganic electrolytes, they are still brit- 

tle and therefore difficult to fit into different battery shapes. Solid-state polymer electrolytes (SSPEs), due to their high flexibility, can fit into any battery shape and present improved safety and stability features compared to crystalline inorganic electrolytes. $\frac{426}{4}$ Since 1980, various high molecular weight, dielectric polymer hosts were investigated for LiBs as high conductivity electrolytes, such as poly(ethylene oxide) (PEO), ${ }^{445}$ polyacrylonitrile (PAN), $\stackrel{46 / 447}{ }$ poly(vinylidene fluoride) (PVDF), 448 450 poly(methyl methacrylate) (PMMA), $\underline{41452}$ and poly(vinylidene fluoride-hexa-fluoropropylene) (PVDF-HFP). 453,455

The ionic conductivities of most polymer electrolytes are significantly lower than those of both oxide solid electrolytes and liquid electrolytes. ${ }^{456}$ A possible solution to this limitation is to create composites by integrating nanoscale, highly conductive, inorganic, particulate fillers into the polymer electrolyte material. $\stackrel{426}{\square}$ This enhances the jonic conductivity and improves the mechanical strength and stability of the SSPEs, including the interfacial stability. $\frac{457}{47}$ Here, heterogeneous doping increases the ionic conductivity as a result of increasing interfacial regions between an inert solid phase, such as silica, alumina, or boron oxide particles, and an electrolyte. $\stackrel{458}{ } \mathrm{~A}$ wide range of inorganic solid composite electrolytes have previously been studied, based on oxides $\left(\mathrm{Li}_{2} \mathrm{O}: \mathrm{Al}_{2} \mathrm{O}_{3}, \stackrel{459}{{ }^{4}} \mathrm{Li}_{2} \mathrm{O}: \mathrm{B}_{2} \mathrm{O}_{3}, \stackrel{460+}{462}\right)$ hydrides $\left(\mathrm{LiBH}_{4}: \mathrm{SiO}_{2}, \stackrel{463}{)}\right)$ halides ( $\left.\mathrm{LiI}: \mathrm{Al}_{2} \mathrm{O}_{3}, \stackrel{464}{,} \mathrm{LiI}: \mathrm{SiO}_{2}, \frac{465}{{ }^{4}} \mathrm{LiF}: \mathrm{Al}_{2} \mathrm{O}_{3},{ }^{466}\right)$ and sulfides $\left(\mathrm{Li}_{2} \mathrm{~S}: \mathrm{SiS}_{2} \cdot{ }^{467}\right)$

Over the last decade, a limited number of candidates with high ionic conductivities $(>1$ $\mathrm{mS} \mathrm{cm}^{-1}$ ) have arisen as potential competitors to liquid electrolytes. ${ }^{468} \sqrt[476]{4}$ Figure 26 presents the ionic conductivities of most currently known solid electrolytes. $\underline{26}$

In this section, we review atomistic modelling investigations into the structure-property relationships in selected solid-state electrolytes: $\mathrm{Li}_{10} \mathrm{GeP}_{2} \mathrm{~S}_{12}$ (LGPS), lithium argyrodites, and $\mathrm{Li}_{7} \mathrm{La}_{3} \mathrm{Zr}_{2} \mathrm{O}_{12}$ (LLZO), belonging to the inorganic solid ceramic electrolyte type, and $\mathrm{Li}_{2} \mathrm{O}: \mathrm{B}_{2} \mathrm{O}_{3}$ materials, belonging to the oxide-based solid composite type. A particular focus is given to the ion transport mechanism in those materials, which is important for reaching high conductivities, a key property of battery materials. Finally, we take a more detailed 2068 look at the interface of solid electrolytes with the electrodes, and discuss the challenges and 


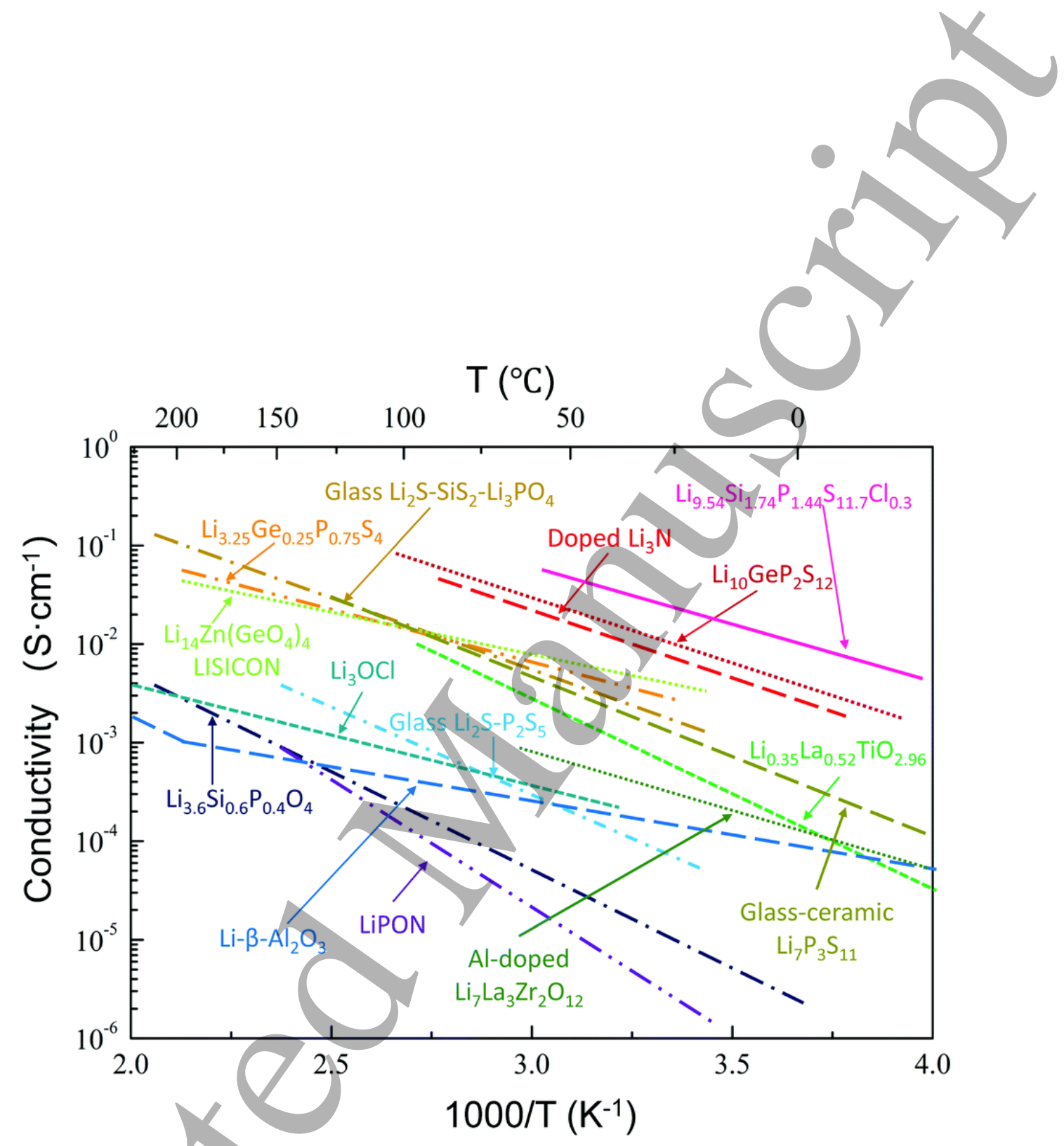

Figure 26: Ion conductivity of several well-known solid lithium ion conductors, including glass and crystalline conductors. Reproduced from Ref. 26- Published by The Royal Society of Chemistry.

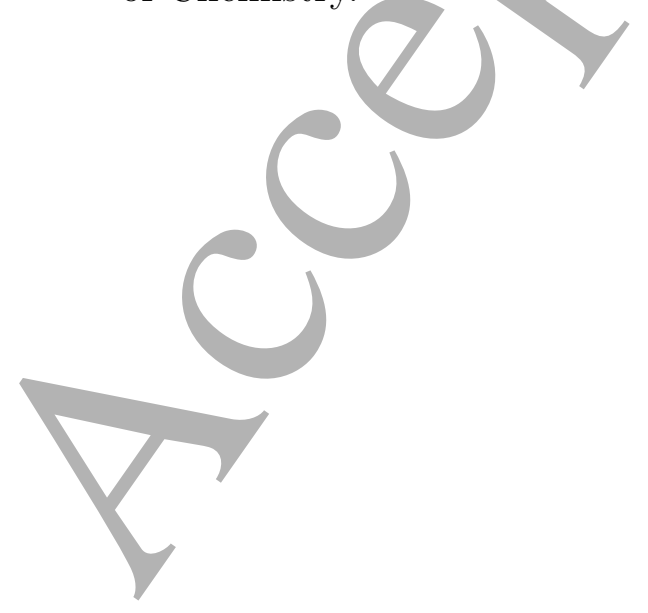


2069

2070

2071

2072

2073

2074

2075

2076

2077

2078

2079

2080

2081

2082

2083

2084

2085

2086

2087

2088

2089

2090

2091

2092

2093

outlook for future atomistic modelling investigations.

\subsubsection{Sulfides}

There are a substantial number of computational studies of sulfides which largely relate to a recent emergence of newly discovered crystalline sulfide superionic conductors. Sulfides also tend to have comparatively lower intrinsic electrochemical and chemical stability, which has stimulated interest in understanding the interfacial interactions within batteries. $\underline{28}$ The sulfide group encompasses a range of sulfide-based solid electrolytes, including glass

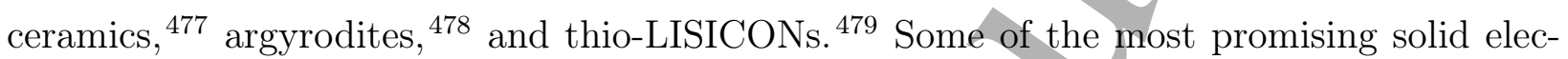
trolytes to emerge in recent years include LGPS, 2881480481 and the Li-argyrodite $\left(\mathrm{Li}_{6} \mathrm{PS}_{5} X\right.$, $X=\mathrm{Cl}, \mathrm{Br}, \mathrm{I})^{442 \sqrt{482} 486}$ families of superionic conductors.

LGPS A study by Kamaya et al. reports that LGPS can reach high room temperature ionic conductivities of $12 \mathrm{mS} \mathrm{cm}^{-1}$, comparable to that of commercial liquid electrolytes $(\sim$ $\left.10 \mathrm{mS} \mathrm{cm}{ }^{-1}\right) .288$ The authors also determined that diffusion in LGPS is anisotropic, where $c$ directional motion is predominant over the $a b$ plane, with an overall energy barrier for $\mathrm{Li}$

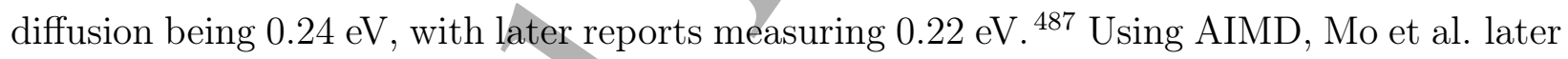
determined the average direction energy/ barriers of $0.17 \mathrm{eV}$ along the $c$ channel and $0.28 \mathrm{eV}$ in the cross channel ( $a b$ plane), $\frac{481}{1}$ with $\mathrm{Xu}$ et al. showing the Li migration mechanism is through cooperative motion, instead of the initially determined single hop mechanism. 488 More recently, Adams and Prasada Rao predicted the presence of additional Li sites using

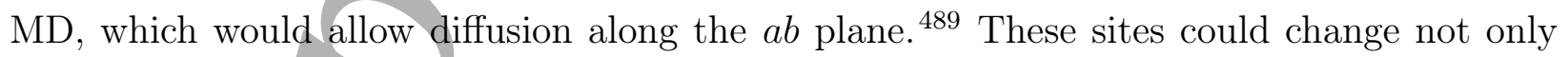
the Li occupancies in the $c$ channel, but also provide a diffusion mechanism involving the $a b$ plane, opening up the possibility of cross-channel diffusion. The presence of these additional sites were later confirmed experimentally using single crystal X-ray Diffraction (XRD). 490

More recently, Bhandari and Bhattacharya also investigated the lithium diffusion dimensionality in LGPS by performing a DFT study of the lithium diffusion energy barrier, using

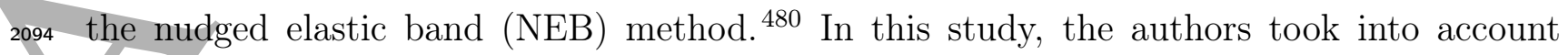


the fractional occupancies leading to variable $c$ channel Li populations, variable chemical environments surrounding Li, and all possible migration mechanisms. The authors found that lithium diffusion is neither purely $c$ directional nor purely along the $a b$ plane, but there exists a correlated mechanism of motion along $c-a b$ which critically controls the degree of anisotropy of Li diffusion in LGPS. The energy barriers for different mechanisms of Lidiffusion, shown in Figure 27, suggest that correlated hopping has the lowest energy barrier. Bhandari and Bhattacharya further performed a statistical average of all diffusion energy barriers, taking into account the formation energy of various Li configurations and predicting an overall energy barrier of $239 \mathrm{meV}$, $\stackrel{480}{ }$ which is in close agreement with experiments. 288 Thus, the DFT approach not only explained the overall diffusivities and energy barriers, but also gave insight into the underlying mechanism behind the fast Li diffusion in LGPS, resolving the discrepancy about the anisotropy of Li diffusion in this compound, which was insight not possible to obtain only from experiments.

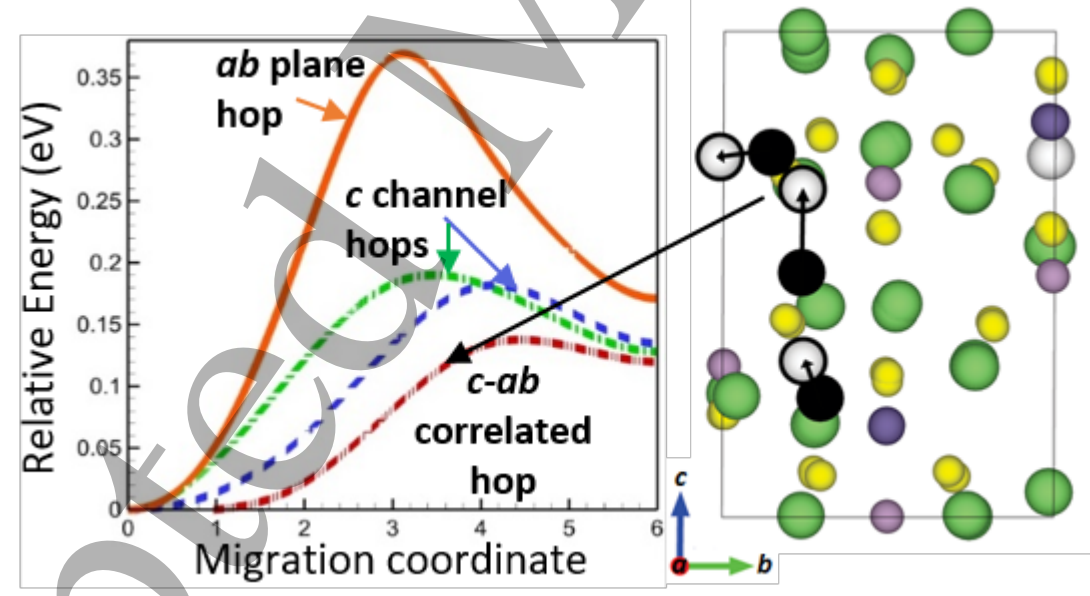

Figure 27: Energy barrier for Li-ion diffusion in the solid electrolyte, $\mathrm{Li}_{10} \mathrm{GeP}_{2} \mathrm{~S}_{12}$ (LGPS), calculated using the nudged elastic band (NEB) method. Reprinted with permission from Ref. 480, Copyright 2016 American Chemical Society.

$2108 \quad$ Lithium argyrodites, $\mathrm{Li}_{6} \mathrm{PS}_{5} X(X=\mathrm{Cl}, \mathrm{Br}, \mathrm{I})$, can reportedly reach ionic conductivities 2109 of up to $10^{-2} \mathrm{~S} \mathrm{~cm}^{-1}{ }^{483}$ While $\mathrm{Li}_{6} \mathrm{PS}_{5} \mathrm{Cl}$ and $\mathrm{Li}_{6} \mathrm{PS}_{5} \mathrm{Br}$ exhibit high ionic conductivities of $211010^{-3} \mathrm{~S} \mathrm{~cm}^{-1}$ at room temperature, $\mathrm{Li}_{6} \mathrm{PS}_{5} \mathrm{I}$ has considerably lower conductivitives of $10^{-6}$ ${ }_{2111} S \mathrm{~cm}^{-1}$. 491 The difference of three orders of magnitude is surprising, as the identical crystal 
2112

2113

2114

2115

2116

2117

2118

2119

2120

2121

2122

2123

2124

2125

2126

2127

2128

2129

2130

2131

2132

2133

2134

2135

2136

2137

structures suggest the same Li diffusion pathways exist in all systems. Another intriguing aspect is that the conductivity trend runs counter to other families of solid electrolytes, such as LGPS, where larger, more polarisable and less electronegative anions are linked with increased ionic conductivites. $\underline{492}$

Understanding which properties and mechanisms influence the conductivity is essential to obtaining higher ionic conductivities and improving battery performance. Material stoichiometry, anion/cation disorder, and doping, have all been shown to influence conductivity. Modification of the lithium stoichiometry has been achieved through aliovalent cation substitution on the $\mathrm{P}$ sites $\frac{482 \sqrt{493}+495}{49}$ and through anion substitution on the $\mathrm{S}^{2-} / \mathrm{X}^{-}$sites. $\frac{486 / 496 / 497}{4}$ For example, Ge substitution on the $\mathrm{P}$ site to give $\mathrm{Li}_{6+x} \mathrm{P}_{1-x} \mathrm{Ge}_{x} \mathrm{~S}_{5} \mathrm{I}$ is able to reach conductivities of up to $5.4 \mathrm{mS} \mathrm{cm}^{-1}$. $482 \mid 495$ The underlying reasons for increased ionic conductivity through cation/anion doping in lithium argyrodites are not clear, with two competing explanations proposed in the literature. 1) Doping increases anion disorder, resulting in percolating networks of lithium diffusion pathways. ${ }^{498}$ 2) Doping increases lithium stoichiometry. The additional lithium is accommodated as "interstitials" which switches on a low-energy concerted diffusion mechanism.

The effects of interrelated factors on ionic conductivity is challenging to resolve purely from experiment. In doped Li-argyrodites there are always both changes to the host-framework (e.g. disorder on anion sites and/or substitution and disorder on the $\mathrm{P}$ sites) and a change in lithium stoichiometry. This is where computational analysis can provide vital insight, allowing deconvolution of coupled properties by modelling non-charge-balanced hypothetical systems to better understand how each of these factors affect ionic conductivity, which is not feasible in experimental systems.

${ }_{2138} 5$ tetrahedral sites in $x(\mathrm{Li})=6$ argyrodites, with occupation of non-type 5 sites only recently 
observed experimentally. $\stackrel{4931500}{ }$ Computational studies, however, have previously predicted occupation of non-type 5 sites, showing lithium distributed over tetrahedral types 5 , 2, and 4.48314981501

Li hopping within these cages, while effectively barrierless, does not contribute/to longrange diffusion. In fact, a combination of inter-cage and intra-cage hopping is needed, with occupation of non-type 5 sites and transitions between all adjacent site types, to achieve long-range diffusion. This is shown schematically in Figure 28, showing the connectivity between the Li tetrahedral sites. AIMD simulations have shown that cation and anion substitution,, $442[493$ anion site disorder, $\underline{498 / 500}$ and lithium concentration $\stackrel{502,504}{504}$ all influence the ionic conductivity.

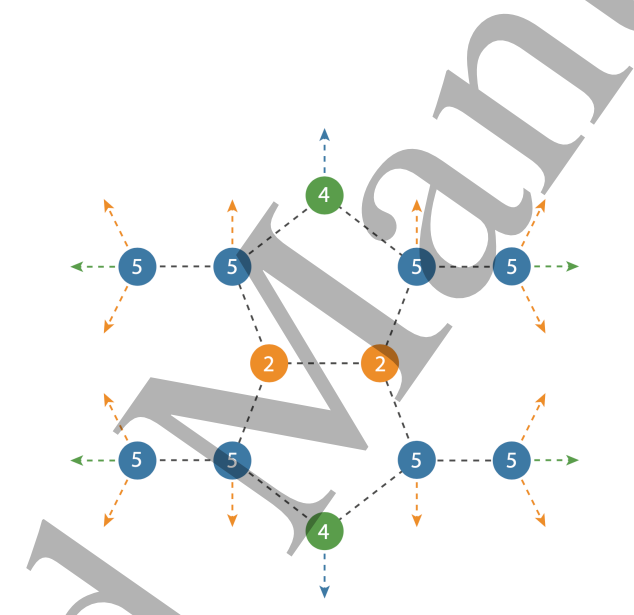

Figure 28: (a) Possible Li diffusion pathways in Li-argyrodites, involving type 2, 4, and 5 tetrahedra for long-range diffusion. Reprinted with permission from Ref. 498. Copyright 2020 American Chemical Society.

The influence of anion substituent concentration on conductivity is currently uncertain, with research by De Klerk et al. determining excess $\mathrm{Cl}$ in $\mathrm{Li}_{5} \mathrm{PS}_{4} \mathrm{Cl}_{2}$, resulting in similar conductivities to $\mathrm{Li}_{6} \mathrm{PS}_{5} \mathrm{Cl},{ }^{442} \mathrm{in}$ contrast to research by $\mathrm{Yu}$ et al. and Feng et al., who concluded that excess $\mathrm{Cl}$ improved Li conductivity. ${ }^{[04505} \mathrm{Yu}$ et al. determined the highest conductivity was produced by $\mathrm{Li}_{5.7} \mathrm{PS}_{4.7} \mathrm{Cl}_{1.3}\left(6.4 \mathrm{mS} \mathrm{cm}{ }^{-1}\right), \underline{5031505}$ while Feng et al. determined this to be $\mathrm{Li}_{5.3} \mathrm{PS}_{4.3} \mathrm{Cl}_{1.7}\left(17 \mathrm{mS} \mathrm{cm}{ }^{-1}\right) . \underline{504}$ Feng et al., however, presented alternative, or coupled, reasoning for this increased conductivity. Drawing from previous studies, $\frac{486[496}{\text { they pro- }}$ posed that the increased $\mathrm{Cl}$ content amplified the anion disorder in the system, which is the 
underpinning cause of the higher conductivities.

\subsubsection{Oxides}

LLZO Cubic $\mathrm{Li}_{7} \mathrm{La}_{3} \mathrm{Zr}_{2} \mathrm{O}_{12}$ (c-LLZO) has a high Li-ion conductivity of $10^{-4} \mathrm{~S} \mathrm{~cm}^{-1}, 506$ a high shear modulus of $59 \mathrm{GPa}, \stackrel{507}{\sqrt{3}}$ and the largest thermodynamic stability window with reference to lithium metal $\frac{24 \mid 508 / 509}{5}$ of current solid electrolyte materials (c.f. section 4.3.4). However, at low temperatures $\left(<150^{\circ} \mathrm{C}\right)$, c-LLZO is not stable and transitions to the less conductive tetragonal LLZO (t-LLZO) phase. ${ }^{510}$ Attempts have been made to retain the more desirable c-LLZO by Al doping on lithium sites, with some success. $\frac{510 \mid 511}{51}$

Lithium dendrite growth has been shown to be a challenge in solid-electrolytes. For LLZO, dendrite growth has caused short circuits in the cells after relatively short peri-

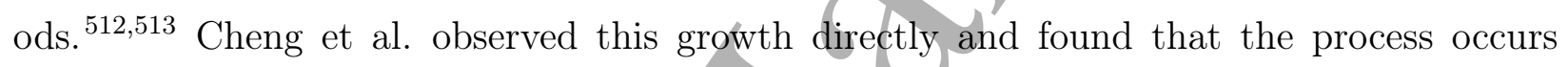
mostly through grain boundaries. $\frac{514}{5 e c e n t l y, ~ K i m ~ e t ~ a l . ~ c o n f i r m e d ~ t h e s e ~ o b s e r v a t i o n s ~ a n d ~}$ investigated the use of an interlayer buffer, to restrict Li propagation through grain boundaries. $\frac{515}{5}$

There has been a wide effort to understand dendrite formation through modelling. $.516,518$ For example, Tian et al. used DFT to investigate dendrite growth through analysis of c-LLZO and t-LLZO bulk and slab surface energies, via the total density of states (TDOS). 517 The authors found that t-LLZO forms at the surface of bulk c-LLZO, even with Al-doping, $\underline{519 \mid 520}$ and that extra states appear in the band gap for the slab structures, which do not appear in the bulk, potentially allowing electrons to be trapped on the surface of LLZO. Electrons localised primarily around $\mathrm{Li}^{+}$and $\mathrm{La}^{3+}$ ions on the surface lead to the nucleation of lithium metal, which can result in lithium growth through grain boundaries and pores in the LLZO, eventually forming dendrites, $\frac{512}{2}$ as shown in Figure 29. This analysis was also conducted on LiPON, where no electron trapping was found to occur, indicating that LiPON could be a suitable coating to prevent dendrite and t-LLZO formation (c.f. section 4.3.4). 


\section{c-LLZO}

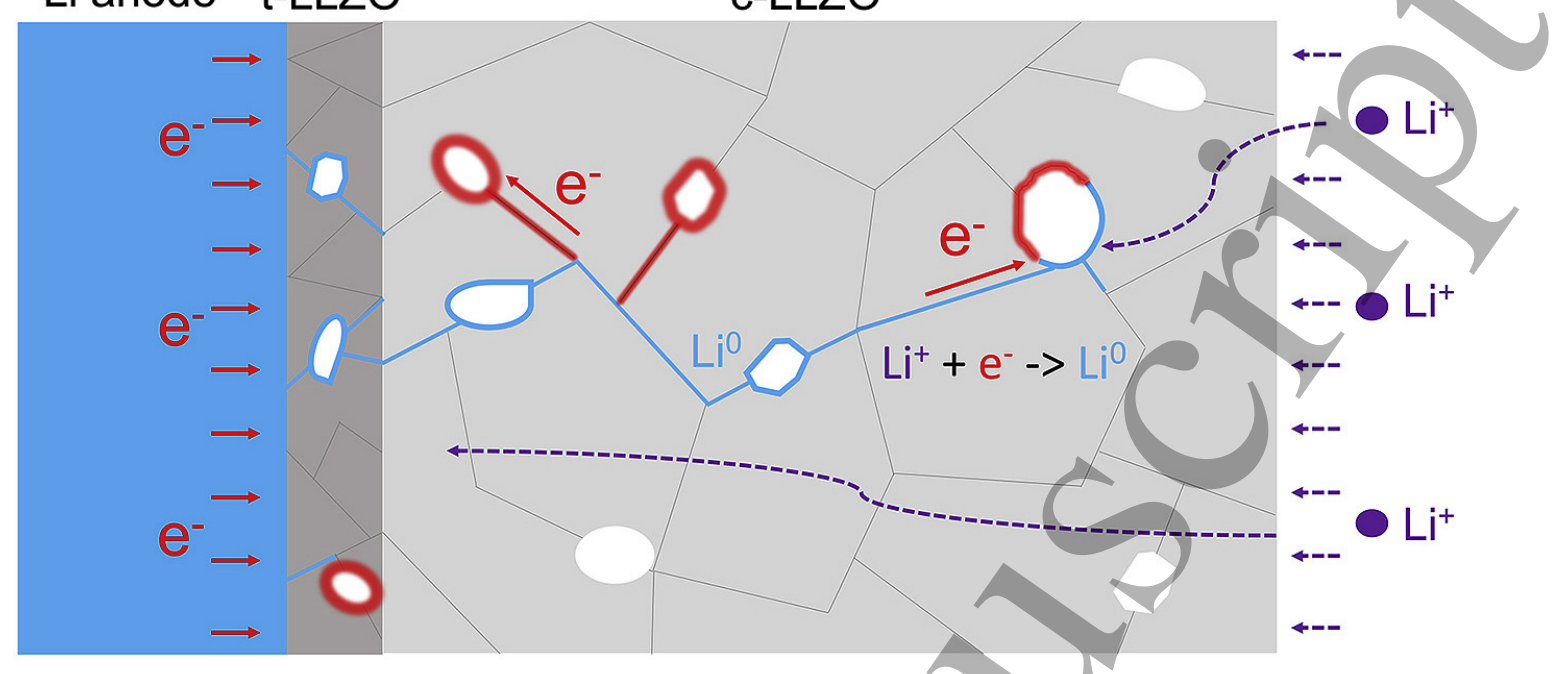

Figure 29: Schematic showing Li metal formation (blue) along grain boundaries and pores, due to electron accumulation (red) combining with $\mathrm{Li}^{+}$as they move through the electrolyte. Reprinted from Ref. 517, Copyright 2018, with permission from Elsevier.

Gao et al. attributed the dendrite growth mechanism to the under-coordination of $\mathrm{Zr}$ present on some of the stable interfaces of LLZO with $\mathrm{Li}, \frac{518}{,}$ leading to inhomogenous $\mathrm{Li}$ depletion, which has been linked to Li metal deposition and dendrite formation. ${ }^{521}[525$ It is unclear whether the suggested cause by Gao et al. is complementary evidence of Tian et al.'s electron trapping theory or a separate cause of interface dendrite growth. However, the papers do differ on their choice of surface. Tian et al. used Li and La rich surfaces, which were determined to be more stable by Thompson et al., who used DFT to investigate 6 different LLZO slabs for the (100) and (110) planes. ${ }^{526}$ By contrast, Gao et al. drew upon results presented in several methods ${ }^{516 \mid 526 / 527}$ and performed DFT calculations on a wider range of surfaces, finding (100) and (001) surfaces to be the most stable. The findings of these studies agree that Li and La rich surfaces are the most stable. However, Gao et al. calculated the interface formation energies of the Li-LLZO interfaces using the CALYPSO interface structure prediction method ${ }^{528}$ and determined the Zr-rich surfaces to be the most stable at this interface. $\frac{529}{}$ Experimental observations corroborate these findings, also determining that the formation of Zr-rich surfaces to be a cause of interfacial degradation. $\frac{524}{52}$

Experimental measurements have suggested a non-uniform distribution of current on the 
surfaces as a possible cause of dendrite growth. $\stackrel{522 / 530}{ }$ Non-uniform current distribution produces random, local spikes in current density for short periods of time, leading to a reduction of Li at these sites. Squires et al. used DFT to model the electronic conductivity in LLZO to probe the importance of the surface current to dendrite formation. ${ }^{531}$ The authors determined that at room temperature, bulk c-LLZO was found to have negligible electron/electron-hole concentrations, indicating that bulk defects are not a significant factor in dendrite growth. However, these models did not account for other forms of defects, such as grain boundary and surface effects.

Understanding Li-ion migration is key to improving battery conductivity. $\mathrm{Xu}$ et al. analysed the Li-ion migration path through LLZO using DFT with the NEB method (c.f. section 2.1.3. ${ }^{532}$ Two migration paths were observed, depending on Li concentration. Low $\mathrm{Li}_{x}\left(\mathrm{Li}_{5} \mathrm{La}_{3} \mathrm{Zr}_{2} \mathrm{O}_{12}\right)$ led to a higher energy, single hop migration path, whereas higher $\mathrm{Li}_{x}$ $\left(\mathrm{Li}_{7} \mathrm{La}_{3} \mathrm{Zr}_{2} \mathrm{O}_{12}\right)$ led to a lower energy, two hop migration path. Using potentials-based MD (c.f. section 2.1.6), Burbano et al. further investigated the Li-ion transport mechanisms by comparing ionic conductivity in t-LLZO and c-LLZO .533 The authors found that the longer time scale of potentials-based MD allowed the observation of a large sample of diffusion events in both LLZO structural forms. Diffusion events in t-LLZO were less common and involved exactly $8 \mathrm{Li}$ ions, which corresponds to the cyclic movement of Li ions around the 12 octahedral and tetrahedral ring sites in t-LLZO. This cyclic mechanism results in no net long-range diffusion of Li and hampers the ability of t-LLZO to conduct ions. AIMD (c.f. section 2.1.6 investigations of the transport mechanism in LLZO have also been conducted. However, the shorter time scale led to some key disagreements about the transport mechanism in c-LLZO. $.533 \lcm{535}$

DFT calculations have determined that $\mathrm{Al}$ doping reduces the energy barrier for Li-ions to move between octahedral and tetrahedral sites, increasing the ionic conductivity $\sqrt{536 / 537}$ More recent work by Bonilla et al., using potentials-based MD, supports this conclusion, finding ${ }_{2224}$ increased conductivity in t-LLZO, due to the Al forcing Li ions into previously inaccessible 
tetrahedral sites. ${ }^{538}$ The authors also found that Al doping in c-LLZO led to a slight decrease in conductivity. They attributed this to the tendency for $\mathrm{Al}$ to "trap" $\mathrm{Li}$ ions close to the dopant. Resolving Li-ion migration through LLZO experimental measurement is challenging due to the complexity of the system coupled with the need to observe the processes during active (dis)charge. While high-level experimental measurements regarding conductivity can be made, which provide spatially-averaged information, $[539$ techniques such as X-ray and neutron diffraction struggle to probe at the atomistic level required for investigating Li-ion migration pathways, due to low ${ }^{7} \mathrm{Li}$ scattering intensities. $\frac{534}{2}$ Other experimental techniques, such as pulsed-gradient NMR, require high-temperature measurements ${ }^{[540}$ and electrochemical impedance spectroscopy (EIS) often includes non-Li charge carriers such as protons or electrons. $\frac{541}{\text { Brugge et al. }}$ used an ion exchange method with focused ion beam secondary ion mass spectrometry (FIB-SIMS) to directly óbserve ion transport occurring through LLZO .541 The authors noted that the sensitivity of LLZO surfaces to carbon contaminants from the air is problematic and leads to large interfacial resistances. $\frac{541542]}{}$ Atomistic modelling does not have the same hindrances stated above and was able to provide a mechanistic pathway to explain the reduced ionic-conductivity of t-LLZO and a thermodynamic description of the effect of Al-doping on ionic conductivity.

Oxide Nanocomposites Due to attractive mechanical, electrical, optical, and magnetic properties, nanocomposite oxide materials represent a new generation of advanced materials. $\frac{4581460}{2}$ They often show enhanced conductivity, compared to single-phase ceramic oxides, making them suitable candidates as electrolytes for future ASSBs. For example, $\mathrm{Li}_{2} \mathrm{O}: \mathrm{B}_{2} \mathrm{O}_{3} \frac{460}{462}$ and $\mathrm{Li}_{2} \mathrm{O}: \mathrm{Al}_{2} \mathrm{O}_{3}$ nanocomposites ${ }^{459}$ have higher ionic conductivities than nanocrystalline $\mathrm{Li}_{2} \mathrm{O}$, although $\mathrm{B}_{2} \mathrm{O}_{3}$ and $\mathrm{Al}_{2} \mathrm{O}_{3}$ are insulators. The ionic conductivity shows a maximum at about $50 \%$ of $\mathrm{B}_{2} \mathrm{O}_{3} / \mathrm{Al}_{2} \mathrm{O}_{3}$ content. This surprising behaviour was attributed to the increased fraction of structurally disordered interfacial regions and the enhanced surface area of the nanosized particles. $\frac{460}{4}$ The oxide nanocomposites contain three types of interfaces; as presented in Figure 30 (a): interfaces between the ionic conductor grains (green 
lines), between the insulator grains (black lines), and between the ionic conductor and the insulator grains (red lines). The latter can lead to surprising effects in the conductivity of composite materials. In this case, the highly conducting interface region can act as a bridge between two $\mathrm{Li}_{2} \mathrm{O}$ grains not in direct contact with each other, opening up additional paths for Li ions. The conductivity enhancement in the interfacial regions may have different origins, e.g. the formation of space charge layers, an enhanced concentration of dislocations, or defects, or the formation of new phases.

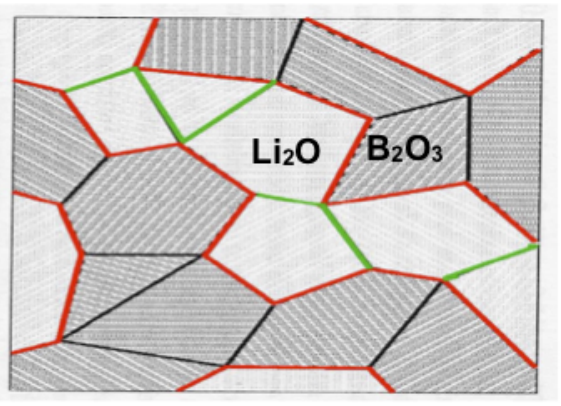

(a)

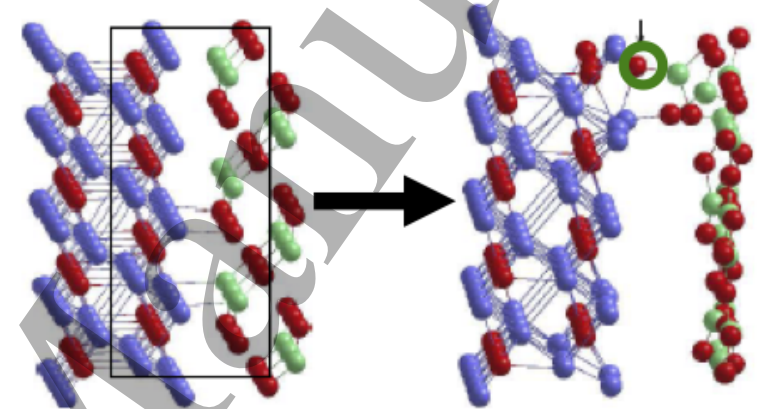

$\mathrm{Li}_{2} \mathrm{O}(111) \mathrm{B}_{2} \mathrm{O}_{3}(001) \quad \mathrm{Li}_{2} \mathrm{O}: \mathrm{B}_{2} \mathrm{O}_{3}$ nanocomposite

(b)

Figure 30: (a) Schematic diagram of $\mathrm{Li}_{2} \mathrm{O}$ and $\mathrm{B}_{2} \mathrm{O}_{3}$ interface (b) Atomistic model of $\mathrm{Li}_{2} \mathrm{O}: \mathrm{B}_{2} \mathrm{O}_{3}$ nanocomposite. Reproduced, with permission from Ref. 543 Copyright IOP Publishing. All rights reserved.

Islam et al. studied the interface of $\mathrm{Li}_{2} \mathrm{O}: \mathrm{B}_{2} \mathrm{O}_{3}$ nanocomposite, by modelling a combination of two favorable surfaces of $\mathrm{Li}_{2} \mathrm{O}$ and $\mathrm{B}_{2} \mathrm{O}_{3}$ using $\mathrm{HF} /$ DFT Hybrid approach. 5431544 After full structural optimisation, it was observed that $\mathrm{Li}-\mathrm{O}$ bonds are weakened, while $\mathrm{B}-\mathrm{O}$ bonds are formed simultaneously at the boundary between the two surfaces, Figure 30 (b). An oxygen atom from the $\mathrm{Li}_{2} \mathrm{O}$ surface (marked by a green circle) is pulled from the surface layer towards a neighbouring boron atom of the $\mathrm{B}_{2} \mathrm{O}_{3}$ surface. This preference of oxygen bonding with $\mathrm{B}$ ( or $\mathrm{Al}$ in $\mathrm{Li}_{2} \mathrm{O}: \mathrm{Al}_{2} \mathrm{O}_{3}$ ) plays a key role in generating low-coordinated Li. As a consequence of this dislocation, the coordination of a $\mathrm{Li}$ atom in the second layer is 2267 reduced from four to three. 
The defect properties were investigated in the interface region. It was observed that the removal of surface oxygen from $\mathrm{Li}_{2} \mathrm{O}$ is responsible for the increased vacancy defect concentration in $\mathrm{Li}_{2} \mathrm{O}: \mathrm{B}_{2} \mathrm{O}_{3}$ (or $\mathrm{Li}_{2} \mathrm{O}: \mathrm{Al}_{2} \mathrm{O}_{3}$ ) nanocomposite materials. Therefore, the nanocomposites of ionic compounds (containing weakly bound and therefore mobile cations) with highly/covalent compounds (with strong metal- or nonmetal-oxygen bonds) are promising candidates for high ionic conductivity. The model calculations showed that the most likely mechanism for $\mathrm{Li}^{+}$migration was in a zigzag pathway, rather than in a straight line along a direction parallel to the interface plane.

The average calculated activation energy for $\mathrm{Li}^{+}$migration in the $\mathrm{Li}_{2} \mathrm{O}: \mathrm{B}_{2} \mathrm{O}_{3}$ interface $(0.28 \mathrm{eV})^{\underline{543 \mid 544}}$ is similar to the experimental values of bulk $\mathrm{Li}_{2} \mathrm{O}(0.31 \mathrm{eV}), \frac{\sqrt[460]{ } \mathrm{Li}_{2} \mathrm{O}: \mathrm{B}_{2} \mathrm{O}_{3}}{}$ $(0.34 \pm 0.04 \mathrm{eV}), \underline{462}$ and $\mathrm{Li}_{2} \mathrm{O}: \mathrm{Al}_{2} \mathrm{O}_{3}(0.30 \pm 0.02 \mathrm{eV})^{459}$ nanocomposites. According to the defect formation energies, the interface region of $\mathrm{Li}_{2} \mathrm{O}: \mathrm{B}_{2} \mathrm{O}_{3}$ nanocomposites contains higher concentrations of both $\mathrm{Li}$ vacancies and Frenkel defects than bulk $\mathrm{Li}_{2} \mathrm{O}$ and $\mathrm{Li}_{2} \mathrm{O}$ surfaces. $\frac{543544}{4}$ Therefore, the experimentally observed enhanced Li mobility in the $\mathrm{Li}_{2} \mathrm{O}: \mathrm{B}_{2} \mathrm{O}_{3}$ interface region is thermodynamically and not kinetically controlled. The models proposed in this study allowed a direct simulation of the defect formation and ion mobility at the atomic scale, without any experimental input. They provide a deep insight into the local bonding situation at the interface of oxide nanocomposites, which is difficult to obtain from experiments. State-of-the-art synchrotron techniques, like hard x-ray photoelectron spectroscopy (HAXPES), could possibly shed light on this challenge. .5451546

\subsubsection{Interface stability}

Experimental investigations of solid electrolyte interfaces are often challenging, making atomistic modelling a vital tool. ${ }^{[29}$ The interfacial stability properties of solid electrolyte materials in contact with an electrode are best described by the electrochemical stability window, defined by Zhu et al. as the range of voltages under which the interface configuration, a mixture 2293 of electrode $(\mathrm{Li})$ and the solid electrolyte, does not undergo a decomposition reaction. $\frac{508}{.}$ 
Atomistic simulation was able to resolve the characterisation of the electrochemical stability window for a number of SSEs. Cyclic voltammetry experiments had reported no significant SSE degradation in contact with a $\mathrm{Li}$ anode for a large $(>5 \mathrm{~V})$ voltage range. $2881547 / 548$ For example, Kamaya et al. used cyclic voltammetry to probe the stability of the LGPS/Li interface for a voltage range of -0.5 to $5 \mathrm{~V} .288$ The authors found no evidence of electrolyte decomposition using this technique, and thus concluded that LGPS has a high electrochemical stability. However, a DFT based investigation by Mo et al. predicted an electrochemical stability window of $3.6 \mathrm{~V}$ for LGPS by simply calculating the band gap of the SSE, rendering an electrochemical stability window $>5 \mathrm{~V}$, as indicated by the cyclic voltammetry results, to be unlikely. $\frac{481}{1} \mathrm{~A}$ more recent DFT study by Zhu et al., posits the formation of an interphase layer, and determining the electrochemical stability window for LGPS to be $0.43 \mathrm{~V}$ (Figure 31). ${ }^{24}$ The authors attribute the apparent contradictory interpretation of the cyclic voltammetry results to the slow kinetics of degradation that occur at SSE/Li interfaces, forming the interphase layer, i.e. there is a large kinetic overpotential for the oxidation and reduction reactions calculated by $\mathrm{Zhu}$ et al. ${ }^{24}$ Cyclic voltammetry is also only able to detect electrochemical degradation processes, in which electrons are transferred. These experiments are unable to detect purely chemical processes (i.e. no electron transfer), which may also occur in parallel with electrochemical processes and lead to erroneous conclusions of "stability" of solid electrolytes through cyclic voltammetry.

A smaller thermodynamic window increases the importance of the interphase layer formation. Zhu et al. determined that a range of solid electrolytes are unstable with respect to Li metal at low and high voltages, with the exception of LLZO, which appears to be kinetically stabilised at low voltages, due to an unfavourable reduction energy of $-0.02 \mathrm{eV}$ per atom. Any potential outside of the thermodynamic stability window results in decomposition into lithium binary compounds, unless otherwise kinetically stabilised. This is problematic for germanium- and titanium-containing compounds, as they form electronically conductive alloys upon decomposition. ${ }^{24}$ This renders the passivation process, proposed by Mo et al., ${ }^{481549}$ 
2321 2322

2323

2324

2325

as the cause of cyclic voltammetry overestimates, unlikely as this degradation process would be sustained throughout the bulk cycling, severely limiting the efficacy of these materials as electrolytes. ${ }^{24}$ Such degradation can also increase interfacial resistance $[\sqrt[5501551]{5}$ Other solid electrolytes face different problems. As explained in section 4.3.3, LLZO forms the far less ionically conductive tetragonal LLZO at the surface. The Li-LiPON and Li-argyrodite interfaces were reported to degrade favourably, forming an ionically conductive and electronically insulating interphase consisting of $\mathrm{Li}_{2} \mathrm{O}, \mathrm{Li}_{2} \mathrm{~S}, \mathrm{Li}_{3} \mathrm{P}, \mathrm{Li}_{3} \mathrm{~N}$, and LiI. 24

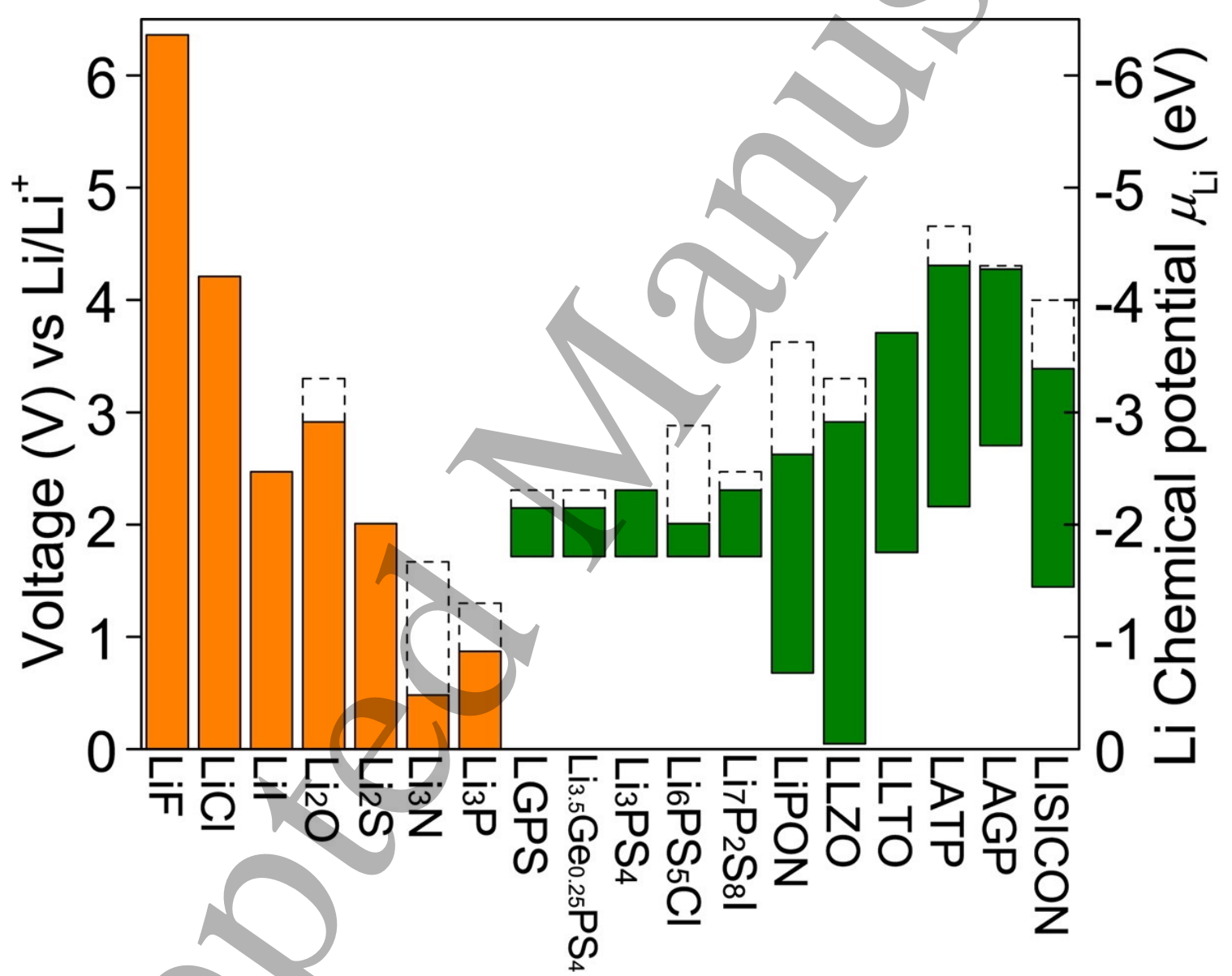

Figure 31: A comparison of the voltage stability windows for a selection of solid electrolytes (green) and the binary compounds that often form upon decomposition of the solid electrolyte (orange). The dashed line represents the oxidation potential to fully delithiate the material. Reprinted with permission from Ref. 24. Copyright 2015 American Chemical Society.

Further study by Zhu et al. sought to investigate the mechanism behind the degrada${ }_{2329}$ tion/instability at the surface. $\frac{508}{}$ In order to probe these mechanisms, the authors calculated 
the chemical and electrochemical stability of several solid electrolytes (LGPS, LLZO, LiPON, NASICON-type, lithium lanthanum titanate oxide (LLTO)) as well as the equilibrium conditions at the interfaces. Examining the cathode-electrolyte interface, using lithium cobalt oxide (LCO) as the cathode, a similar pattern emerged: oxides were found to be far more stable than their sulfide counterparts. However, LLTO and lithium aluminum titanium phosphate (LATP) had the best electrochemical stability against LCO.

Studies looking into the interfacial resistance have been conducted, $301552 / 554$ with the main source of resistance attributed to the electric double layer, which, in liquid electrolytes, consists of a capacitance and diffusion layer (c.f. section 4.1) $\cdot 30$ Tateyama et al. used the CALYPSO method ${ }^{528529}$ to find low-energy surfaces to probe the interface. The lithium chemical potential of these stable interfaces in the Helmholtz layer, corresponding to the negative of the Li ion vacancy formation energy, was determined. These energies correspond to lithium moving from the electrode to the electrolyte, with the vacant lithium sites becoming a potential source of interfacial resistance. Okuno et al. use DFT calculations to compare the interfacial resistances of sulfide and oxide based solid electrolytes with LCO cathodes. .552 The Li vacancy formation energy and ion exchange across various interfaces were calculated. It was found that sulfide-based electrolytes had a higher interfacial resistance, due to the presence of more sites with a low vacancy formation energy on the surface. The authors also found the interfacial resistance to be dependent on the orientation of the crystals at the interface. Interfacial resistance is a major impediment to the commercialisation of ASSBs. The cause of this phenomenon has been elucidated through atomistic simulation of the interface and has provided direction to future SSE development.

A study by Lepley and Holzwarth used DFT to investigate the interface energies between the Li electrode and the compounds that make up the interphase layer of the electrolyte. 555 They defined the interface energy as:

$$
\gamma_{a b}(\Omega)=\frac{E_{a b}\left(\Omega, A, n_{a}, n_{b}\right)-n_{a} E_{a}-n_{b} E_{b}}{A},
$$


where $\Omega$ is the interface configuration of atoms, $E_{a b}$ is the energy of the complete system, $E_{x}$ is the bulk energy per for formula unit and $A$ is the surface energy. Because the interface energy is intensive, calculating larger systems will give a converging value for $\gamma_{a b}$,

$$
\lim _{\Omega_{s} \rightarrow \Omega}\left[\gamma_{a b}\left(\Omega_{s}\right)\right]=\gamma_{a b}(\Omega)
$$

where $\Omega_{s}$ is the atomic configuration in a sample of the interface volume. Because the exact matching of lattice constants between interfaces is unlikely, a semi-coherent interface is considered, meaning lattice strain needed to be taken into account. Using the lowest overall lattice energy structure and explicitly accounting for the lattice strain, the most probable interfaces could be found. The $\mathrm{Li} / \mathrm{Li}_{3} \mathrm{PO}_{4}, \mathrm{Li} / \mathrm{Li}_{2} \mathrm{O}$ and $\mathrm{Li} / \mathrm{Li}_{2} \mathrm{~S}$ interfaces were found to be stable and the $\mathrm{Li} / \mathrm{Li}_{3} \mathrm{PS}_{4}$ interface was found to be unstable. 555

In response to the apparent poor stability of most solid electrolytes, many studies have attempted to simulate the effect of coating the electrolyte with an oxide layer. .51715561557 As discussed in section 4.3.3, Tian et al. identified LiPON as a suitable coating material for LLZO, by comparing the bulk and surface density of states. ${ }^{517}$ The authors found no extra states on the surface structure, so concluded that no electron trapping would occur (the primary mechanism that they attributed to dendrite formation). Recently, Sang et al. proposed an artificial interphase layer between the Li anode and the solid electrolyte, composed of a $\mathrm{Li}_{3 a_{b}} \mathrm{~N}_{a} X_{b}$ compound, where $X$ is a halide. ${ }^{558}$ This material was investigated computationally by screening stable and metastable structures using the USPEX structure prediction software. 5595560 The dynamic stability of the stable structures was found by analysing the

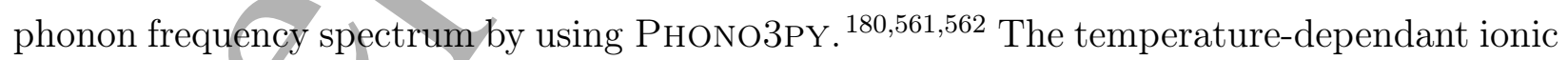
transport properties were found using AIMD (c.f. section 2.1.6).

Phase diagrams for various atomic configurations were then constructed using cluster expansion, implemented through the Alloy Theoretic Automated Toolkit (AT-AT) (c.f. section 2.1.4 801563 Through these various computational techniques, Sang et al. found that $\mathrm{Li}_{6} \mathrm{NCl}_{3}$ has the most favourable properties for use with sulfide-based solid electrolytes, such 
as LGPS. ${ }^{558}$ Authors such as Tian et al. and Sang et al. have leveraged the knowledge of the electronic structure and thermodynamic stability gained through atomistic simulation to predict viable coatings for the SSE. The versatility of computational methods allows for a greater variety of these materials to be analysed and could provide a means to screen potential SSE coating materials before performing costly and time-consuming experimental investigations. $\frac{564}{5}$

\subsubsection{Outlook and challenges}

The drive for the development of commercialised ASSBs has been intense, with the EV industry at the forefront of promoting this. ${ }^{23}$ Although ASSBs can offer high gravimetric energy density (250 Wh $\left.\mathrm{kg}^{-1}\right)$ and volumetric energy density (700 Wh $\mathrm{L}^{-1}$ ), along with improved safety over conventional liquid electrolytes, the slow ionic diffusion can impair fast discharge and charge performance. With solid electrolytes intended to replace both the separator and liquid electrolyte in conventional LiBs, $\frac{565}{5}$ there are still multiple challenges which need to be overcome for this to be viable. In recent years, there have been breakthroughs in the discovery of new solid electrolytes, such as $\mathrm{Li}_{9.54} \mathrm{Si}_{1.74} \mathrm{P}_{1.44} \mathrm{~S}_{11.7} \mathrm{Cl}_{0.3}$, ${ }^{566}$ which exhibit ionic conductivity competitive with that of organic liquid electrolytes. The improved performance of these materials is enabled by interfacial coatings or buffer layers and micro-structure engineering solutions at the electrode/electrolyte interfaces. $\frac{567}{56}$

ASSBs are currently not capable of reliable cycling at current densities $>0.6 \mathrm{~mA} \mathrm{~cm}^{-2} . \underline{424568}$ The current density and stability is limited by: poor electrode/electrolyte physical contact, leading to particle cracking and interface delamination, formation and propagation of $\mathrm{Li}$ dendrites, chemical and electrochemical stability, and high interfacial resistance. ${ }^{424}$ There are several critical issues related to the pairing of solid electrolytes with cathode and anode materials, which need to be addressed for long-term battery operation:

- The limited system sizes of atomistic modelling are not sufficient to capture lattice relaxation, which would allow a coherent (completely matched) interface to form. This 
amplifies the effects of lattice strain in the model, particularly in cases where periodic boundary conditions are used. .555 The lattice strain energy can be calculated and factored into bulk scale calculations, but it is not as accurate as explicitly calculating dislocation defects that naturally relieve lattice strain. $\underline{5691570}$

- Dendrite formation has been a notable problem for even the most physically robust electrolytes (c.f. section 4.3.3). Modelling of dendrite formation mechanisms has yielded

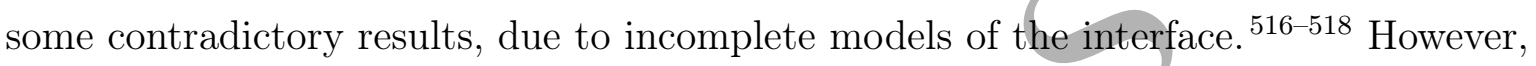
a more detailed understanding requires modelling of larger systems, encompassing the interface and bulk regions of both materials. This incurs a high computational cost not currently reachable through electronic structure methods methods. Further development of the linear-scaling DFT approach (c.f. section 2.1.2) may allow a more complete, multiscale approach.

- The system size limitations in DFT modelling also hinder the modelling of the full electric double layer, which is also applicable to liquid electrolytes. Comparatively, in solid electrolytes the double layer is less understood. For example, Tateyama et al. were only able to successfully model the initial capacitance layer at the interface (Helmholtz layer) $\underline{30}$

- Interfacial resistance presents an interesting challenge, as it can be introduced through multiple mechanisms: ${ }^{\sqrt[554]{4}}$ electric double layer, ${ }^{30}$ surface crystal orientation,,$[552$ and production issues, such as poor wettability. $\frac{[53]}{5}$ Strong collaboration between theorists and experimentalists will be needed, in order to make informed improvements to current interfacial structures.

The interface is the primary source of dendrite formation, lattice mismatch, and interfacial resistance in solid electrolytes. The interface also presents opportunities for atomistic modelling, with the growing popularity of coatings that try to address the shortcomings of 2431 popular solid electrolytes. ${ }^{29|515| 571 \mid} 584 \mid$ For example, Tian et al.'s solution to dendrite growth 
2432

2433

2434

2435

2436

2437

2438

2439

2440

2441

in LLZO by utilising a LiPON coating $\sqrt[517]{ }$ (c.f. section 4.3.3). Understanding how effective coatings are at addressing the aforementioned issues is essential. $517|556| 557$ A very recent review by Kim et al. presents a detailed insight into the challenges and future prospects of solid-state Li-metal batteries, which we have touched upon here. 567

\section{Cathodes}

\subsection{Introduction}

As mentioned in our Introduction (section 1), lithium-ion batteries (LiBs) became promising applications in 1979 when Goodenough and Mizushima successfully demonstrated $\mathrm{LiCoO}_{2}$ as a cathode. ${ }^{585}$ Since then, LiBs have become instrumental in portable electronics, such as mobile phones, and electric vehicles, ${ }^{586}+590$ largely attributed to their high energy den-

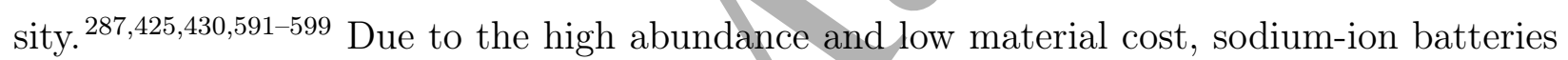
have also received increased attention, especially for grid storage applications. $\frac{600}{604}$ Regardless of the application, the discovery of new materials and the optimisation of current chemistries for improved performance is crucial for the next generation of rechargeable batteries. With that in mind, it is known that the energy density of the cathode material is the limiting factor in improving battery performance, thus current research is largely focused on exploring cathode chemistries. These include layered oxides $\left(\mathrm{Li} M \mathrm{O}_{2}, M=\mathrm{Co}, \mathrm{Mn}, \mathrm{Ni}\right)$, spinel oxides $\left(\mathrm{LiM}_{2} \mathrm{O}_{4}\right)$, olivine phosphates $\left(\mathrm{LiFePO}_{4}\right)$, disordered rock-salts, $\left(\mathrm{Li}_{2} \mathrm{MnO}_{2} \mathrm{~F}\right)$, and other compounds, such as silicates. 6051606

Layered transition metal (TM) oxides $\left(\mathrm{Li} M \mathrm{O}_{2}, M=\mathrm{Co}, \mathrm{Mn}, \mathrm{Ni}\right.$,etc.) are commonly considered to be the first generation of cathode materials in commercial LiBs. These materials possess a theoretical specific capacity of $270 \mathrm{mAh} \mathrm{g}^{-1}$. However, their practical capacity is generally limited to below $200 \mathrm{mAh} \mathrm{g}^{-1} \cdot \frac{607}{\mathrm{LiCoO}_{2}}$ held high capacities but the material was problematic due to capacity fading, low abundance, and the high cost of cobalt and geopolitical issues, including ethical concerns, making large scale applications impractical. $\frac{608}{6 h e r e}$ 

is also considerable instability in the $\mathrm{LiCoO}_{2}$ structure, caused by the extraction of $\mathrm{Li}$ during cycling, which results in undesirable phase transitions from O3-type to O6-type $\mathrm{Li}_{x} \mathrm{CoO}_{2}$ and O1-type $\mathrm{CoO}_{2} \cdot \frac{609610}{6 t h e r}$ layered oxides also pose their own challenges, such as $\mathrm{Li}_{x} \mathrm{NiO}_{2}$ presenting capacity fade and poor safety, ${ }^{611}$ and $\mathrm{Li}_{x} \mathrm{Mn}_{2} \mathrm{O}_{4}$ presenting low capacity. $\frac{612}{6} \mathrm{An}$ emerging alternative to solve some of these challenges is using a combination of the TMs. In 2000, Paulsen et al. presented $\mathrm{Li}_{2 / 3}\left[\mathrm{Ni}_{1 / 3} \mathrm{Mn}_{2 / 3}\right] \mathrm{O}_{2}, \frac{613614}{}$ with $\mathrm{Li}\left[\mathrm{Ni}_{x} \mathrm{Mn}_{1-2 x} \mathrm{Co}_{z}\right] \mathrm{O}_{2}(\mathrm{NMC})$ presented by the authors in 2001.615 Partially replacing $\mathrm{Co}$ in $\mathrm{LiCoO}_{2}$ with $\mathrm{Ni}$ and $\mathrm{Mn}$ to obtain layered $\mathrm{Li}\left[\mathrm{Ni}_{x} \mathrm{Mn}_{y} \mathrm{Co}_{z}\right] \mathrm{O}_{2},{ }^{586}$ where $x+y+z=1$, shows improved electrochemical performance, while also reducing material cost and improving stability. ${ }^{616}$ These layered oxides are commonly termed as NMC, with the subsequent numbering relating to the ratio between the cations.

A huge benefit of combining these TMs is the ability to tune the TM composition to optimise aspects including capacity, (dis)charging rate, electrochemical stability, and lifetime, with the potential of reaching capacities $>220 \mathrm{mAh} \mathrm{g}^{-1}$.617 Some NMC compositions are already used commercially, with industry focus shifting from NMC111 to higher Ni containing compositions including NMC442, NMC532, and NMC622. ${ }^{618}$ These compositions, however, still contain $20 \%$ or more Co. A great deal of research is working towards reducing the Co content even further, with compositions such as NMC811 $\left(\mathrm{Li}\left[\mathrm{Ni}_{0.8} \mathrm{Mn}_{0.1} \mathrm{Co}_{0.1}\right] \mathrm{O}_{2}\right)$ showing promise as future commercial materials for applications, such as in long-range electric vehicles (EVs).619 These Ni-rich NMC compositions are also considered to be the cathode of choice for future all-solid-state LiBs. .607

Recently, research into further improving the capacity of these materials by inserting lithium into the TM cation sites has attracted considerable attention. This has lead to a new generation of cathode materials termed "Li-rich" or lithium excess. The increased capacities of these materials arises from invoking redox chemistry on both the TM and oxide ions, as opposed to just TM ions in traditional oxide-based intercalation compounds. $620 \mid 628$ These Li-rich cathodes, including $\mathrm{Li}_{1+x} \mathrm{Ni}_{y} \mathrm{Co}_{z} \mathrm{Mn}_{(1-x-y-z)} \mathrm{O}_{2}$ layered oxide, can reach high 
capacities of $>300 \mathrm{mAh}^{-1}$. However, synthesis of these materials has proven to be difficult and work is ongoing to improve synthesis techniques. $\frac{629}{6}$

There has also been growing interest in disordered intercalation structures, especially disordered rock-salt structures. They were initially disregarded as cathodes, as their structure appeared to limit lithium diffusion. However, recent research has shown that lithium diffusion can be facile in some disordered materials, provided that there is enough of a lithium excess to allow the formation of an uninterrupted percolating network of channels involving no face-sharing TM ions. $\frac{621 / 630 / 631}{1}$ There have been several examples reported, including $\mathrm{Li}_{1.2} \mathrm{Ni}_{0.33} \mathrm{Ti}_{0.33} \mathrm{Mo}_{0.13} \mathrm{O}_{2},{ }^{631} \mathrm{Li}_{1.2} \mathrm{Ti}_{0.4} \mathrm{Mn}_{0.4} \mathrm{O}_{2},{ }^{632} \mathrm{Li}_{4} \mathrm{Mn}_{2} \mathrm{O}_{5}, \stackrel{633}{\underline{635}} \mathrm{Li}_{3} \mathrm{NbO}_{4}$-based systems, $\underline{636} \underline{638}$ and oxyfluorides, where some of the anion sites are occupied by $\mathrm{F}^{-}$rather than $\mathrm{O}^{2}-$, such as $\mathrm{Li}_{2} \mathrm{MnO}_{2} \mathrm{~F}, \underline{639} \underline{641} \mathrm{Li}_{2} \mathrm{VO}_{2} \mathrm{~F}, \underline{642} \underline{648}$ and $\mathrm{Li}_{2} \mathrm{Mn}_{2 / 3} \mathrm{Nb}_{1 / 3} \mathrm{O}_{2} \mathrm{~F}$. $\underline{649}$ These materials can be difficult to synthesise, however, as Mn-rich 3D TM compounds tend to form ordered phases, such as $\mathrm{LiMnO}_{2}$ or $\mathrm{Li}_{2} \mathrm{MnO}_{3}$, high energy mechano-chemical ball-milling methods have been utilised to counter this. $\frac{633640 / 650}{6}$ These materials are able to reach very high energy storage capacities of $300 \mathrm{mAh} \mathrm{g}^{-1}, 651$ which is attributed to the ability to perform both cationic and anionic redox. $\frac{648651652}{6}$ These materials typically show less first cycle hysteresis than other Li-rich compounds, thought to be because the structure already resembles that of the Li-rich materials after they undergo cation disorder on cycling.

Knowledge of the broad structural and electrochemical properties of cathode materials can be obtained from various experimental methods. However, detailed insight into, for example, TM configurations, vibrational and thermal properties, and atomistic diffusion mechanisms, is challenging and, in some cases, not resolvable using experimental techniques. This is where atomistic modelling can provide greater insight. In this section, we explore a range of cathode material properties, using several Li-ion materials, to highlight different properties and the considerations needed to gain the most desirable electrochemical performance. We describe which atomistic modelling methods are used to investigate the discussed 2510 properties and the importance of modelling in this context. Using a range of promising cath- 
2511

2512

2513

2514

2515

2516

2517

2518

2519

2520

2521

2522

2523

2524

2525

2526

2527

2528

2529

2530

2531

2532

2533

2534

2535 2536

ode materials (layered oxides, spinel oxides, polyanions, and disordered rock-salt oxides and oxyfluorides) to aid in the discussion, we first look at the different cathode crystal structures and the effects of micro-structuring. We then discuss some of the bulk material properties, including ion diffusion, redox and electronic properties, TM ordering, and vibration and thermal properties. Finally, we consider the surfaces and interfaces of these cathode materials, with an outlook to current and future challenges in the atomistic modelling of cathodes.

\subsection{Bulk Properties}

\subsubsection{Crystal Structure and Micro-Structure}

Crystal structure. Cathode materials consist of a range of different crystal structures, with some of the most promising $\mathrm{LiCoO}_{2}$ based materials adopting the $\alpha-\mathrm{NaFeO}_{2}$ structure, with alternating layers of $\left[\mathrm{CoO}_{2}\right]^{-}$and $\mathrm{Li}^{+}$. In $\mathrm{LiBs}$, the cathode is a limiting factor, as the amount of lithium that can be reversibly extracted and re-inserted (cycled) directly influences the battery capacity, with the Fermi energy linked to the cell voltage. ${ }^{606}$ Thermo-chemical stability and high energy density are also important considerations, with several promising candidates for future battery materials. These include mixed-metal layered oxides (NMC), spinel oxides

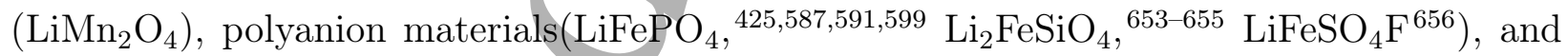
disordered rock-salt oxides and oxyfluorides $\left(\mathrm{Li}_{2} \mathrm{MnO}_{2} \mathrm{~F}^{639164816516521657}\right)$. The crystal structures of these cathode materials are presented in Figures 32 and 33 , where these materials are described in more detail.

Some TM oxides are stable in various structural forms, such as lithium manganese oxide (LMO), which has been synthesised with layered, $\frac{658}{65 i n e l, ~} \frac{659}{6}$ and rock-salt structures. $\frac{660}{6}$ For intercalation-type cathodes used in LiBs, the structural framework is expected to remain relatively unchanged, with only small changes from lattice expansion/contraction. However, phase transitions can occur during the cycling process. For example, during cycling, a phase transition can occur from the $\mathrm{LiMn}_{2} \mathrm{O}_{4}$ spinel structure to the $\mathrm{LiMnO}_{2}$ rock-salt structure, partially due to oxygen evolution. $[61]$ Phase transitions between layered and spinel struc- 

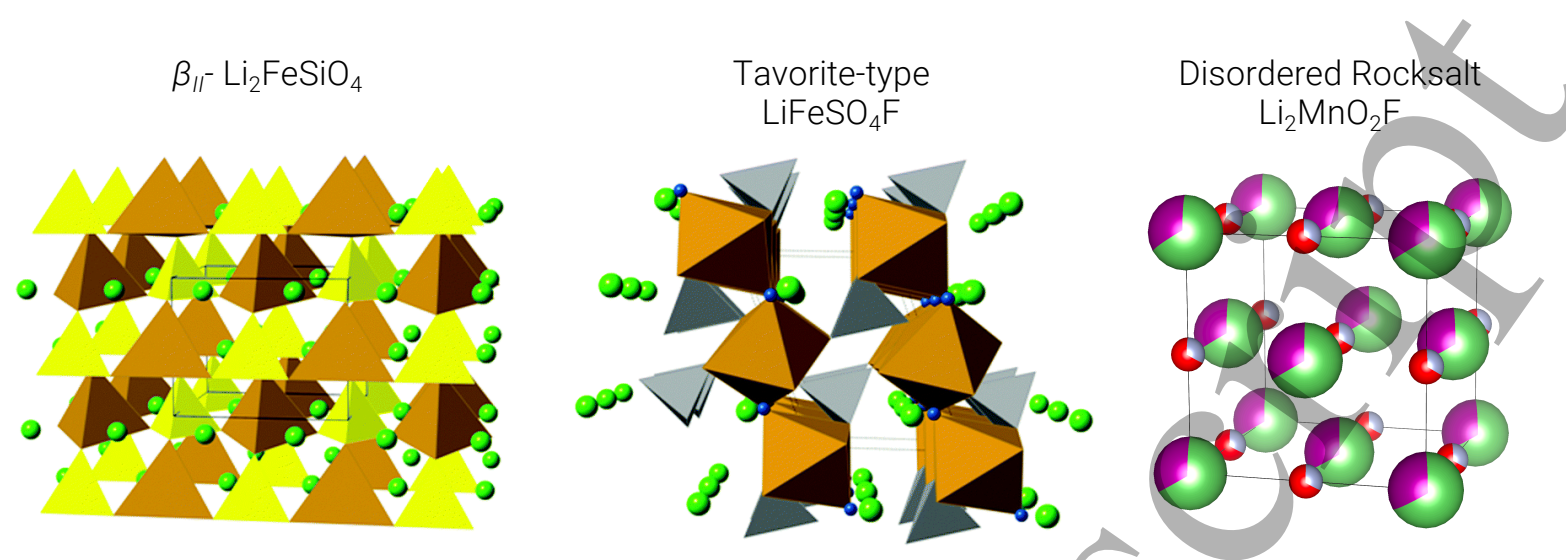

Figure 32: Representative crystal structures of $\beta_{I I}-\mathrm{Li}_{2} \mathrm{FeSiO}_{4}$, tavorite-type $\mathrm{LiFeSO}_{4} \mathrm{~F}$, and disordered rock-salt $\mathrm{Li}_{2} \mathrm{MnO}_{2} \mathrm{~F}$ cathode materials for lithium-ion batteries. $\mathrm{Li}^{+}$ions are shown in green spheres, $\mathrm{O}$ in red, $\mathrm{Mn}$ in mauve, and $\mathrm{F}$ in grey. Fe $\mathrm{O}$ polyhedra are shown in brown, $\mathrm{SiO}_{4}$ tetrahedra in yellow, and $\mathrm{SO}_{4}$ tetrahedra in grey.

tures are also widely observed ${ }^{662}$ For example, Reed et al. investigated the layered to spinel phase transitions in $\mathrm{Li}_{x} \mathrm{MnO}_{2}$ using Density Functional Theory (DFT) modelling (c.f. section 2.1.1). $\frac{663}{6}$ Their investigation determined that partially lithiated layered $\mathrm{Li}_{x} \mathrm{MnO}_{2}$ transitions to spinel in a two-stage process. Firstly, a large percent of Mn and Li ions quickly occupy tetrahedral sites, to form a meta-stable intermediate. Then, a more complex, coordinated rearrangement of $\mathrm{Mn}$ and Li occurs to form spinel. Interestingly, this behaviour is in contrast to $\mathrm{Li}_{x} \mathrm{CoO}_{2}$ and understanding, the reasons for this could prove useful for creating Mn-based cathode materials.

Micro-Structuring. It is clear that control over bulk structure has an impact on the material's performance, as many properties are dependent on shape and size $\frac{664}{64}$ The structural and micro-structural properties of a material are also vital to the cycling stability of a cathode. For example, reducing the particle size of $\mathrm{LiFePO}_{4}$ to the nanometre scale is shown to increase the electrochemical performance, compared to equivalent, but larger, particles, by reducing transport path lengths. $\frac{665}{667}$ Selective structuring can also provide mechanical benefits, for example, where forces acting on the functional cathode during cycling, as the lattice expands and contracts with lithium intercalation, can cause plastic deformation and extinguish desirable activities. Ledwaba et al. modelled diffusion-induced stress in layered-spinel 
LMO composites, revealing structural resilience, enabled by flexing of a porous structure. $\frac{668}{6}$ In this study, Ledwaba et al. found the yield stress of the bulk material was $11.35 \mathrm{GPa}$, whilst the nanoporous material subjected to an equivalent strain experienced a stress of 4.32 GPa. In fact, it has been proposed that a $\beta-\mathrm{MnO}_{2}$ host should be symmetrically porous and heavily twinned to maximise the cathode's electrochemical properties. $\frac{669}{9}$ Further to this, intergrowing structures of two polymorphs of $\mathrm{MnO}_{2}, \beta-\mathrm{MnO}_{2}$ and Ramsdellite- $\mathrm{MnO}_{2}, \frac{670}{6}$ has been shown to enhance cell performance, ${ }^{671}$ due to reduction in stresses and facile diffusion in more open structure of Ramsdellite- $\mathrm{MnO}_{2}$.

\subsubsection{Lithium-ion Diffusion}

As discussed in section 2.3.3, Li-ion diffusion coefficients can be calculated using multiple techniques, including ab initio Molecular Dynamics (MD), classical (potentials-based) MD, and Monte Carlo (MC). Diffusion coefficients, although important experimentally and for parameterising continuum models, are not the only ion transport property of interest on the atomistic scale. Properties such as atomistic diffusion mechanisms, hopping frequencies, and activation energy barriers are all vital to understanding Li-ion transport and (dis)charge rate behaviour. This is of particular interest for investigating the effects of grain-boundaries and interfaces on the migration routes and mechanisms. For example, in $\mathrm{LiCoO}_{2}$, Moriwake et al. determined that the activation energy, $E_{a}$, for Li migration along a twin boundary is $0.20 \mathrm{eV}$, smaller than that in the bulk, while the $E_{a}$ across a twin boundary is $0.4 \mathrm{eV} \cdot \underline{672}$ This demonstrates the influence of grain-boundaries on the kinetic properties.

Computational techniques can provide information regarding a material's diffusion behaviour, which cannot be fully understood through experiments alone. For example, Dixit et al. compared $\mathrm{Li}$ and $\mathrm{Na}$ diffusion in $\mathrm{Li}_{0.25} \mathrm{FePO}_{4}$ and $\mathrm{Na}_{0.25} \mathrm{FePO}_{4}$, respectively, by calculating the potential and free energy diffusion barriers and determining the nuclear quantum effects (NQEs) of the Li ions. $\frac{673}{6}$ Their calculations found that Li diffusion was faster than Na diffusion, which is in agreement with experiments. However, the authors also determined 
that the NQEs for Li-ions were higher than those for Na-ions and that the quantum behaviour of the Li-ions was unusual. This information would not be possible to resolve using current experimental methods.

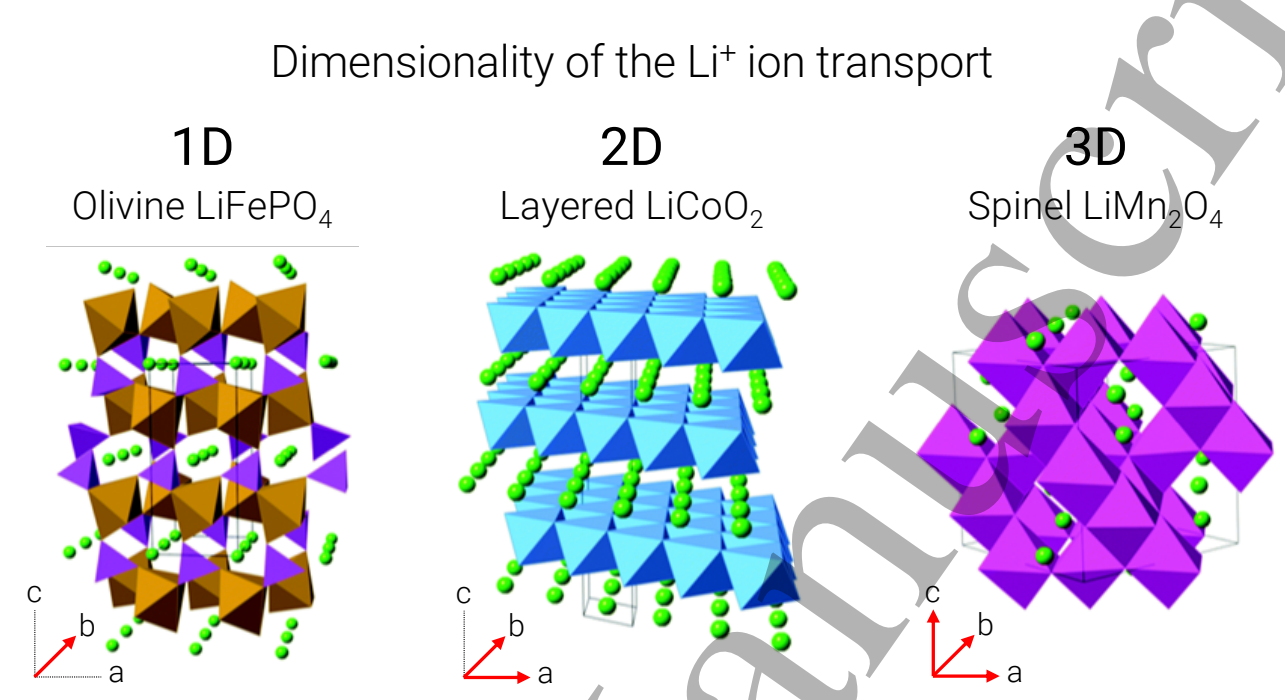

Figure 33: Dimensionality of the $\mathrm{Li}^{+}$ion diffusion in $\mathrm{LiFePO}_{4}, \mathrm{LiCoO}_{2}$, and $\mathrm{LiMn}_{2} \mathrm{O}_{4}$. Figure edited and reproduced with permission from Ref. 606 - Published by The Royal Society of Chemistry.

The cathode crystal structure determines the available diffusion pathways in the material. DFT calculations ${ }^{\sqrt[674675]{67}}$ and classical MD using a core-shell model ${ }^{676}$ show $\mathrm{Li}_{x} \mathrm{FePO}_{4}$ is an olivine based structure which hosts Li over an interstitial network that has one-dimensional connectivity, i.e. 1-D diffusion, along the $b$ lattice vector of the orthorhombic cell. ${ }^{677} \mathrm{Li}_{x} \mathrm{CoO}_{2}$ is a layered compound that aecommodates $\mathrm{Li}$ ions within octahedral sites forming twodimensional triangular lattices, resulting in 2-D diffusion, along the $b$ and $c$ lattice vector of the orthorhombic cell. ${ }^{678}$ The spinel form of $\mathrm{Li}_{x} \mathrm{Mn}_{2} \mathrm{O}_{4}$ has both tetrahedrally and octahedrally coordinated Li interstitial sites, forming a three-dimensional network and resulting in 3-D diffusion, along all lattice vectors. $\frac{679680}{6}$ These different diffusion pathways can bee seen in Figure 33. The 1-D diffusion pathways in $\mathrm{Li}_{x} \mathrm{FePO}_{4}$ are not actually exactly one dimensional. Although they travel solely along the $b$ lattice vector, the pathways themselves 2594 are curved, as shown in Figure 34, as originally predicted by Islam et al. using atomistic 2595 modelling, ${ }^{676}$ before later being observed experimentally. 

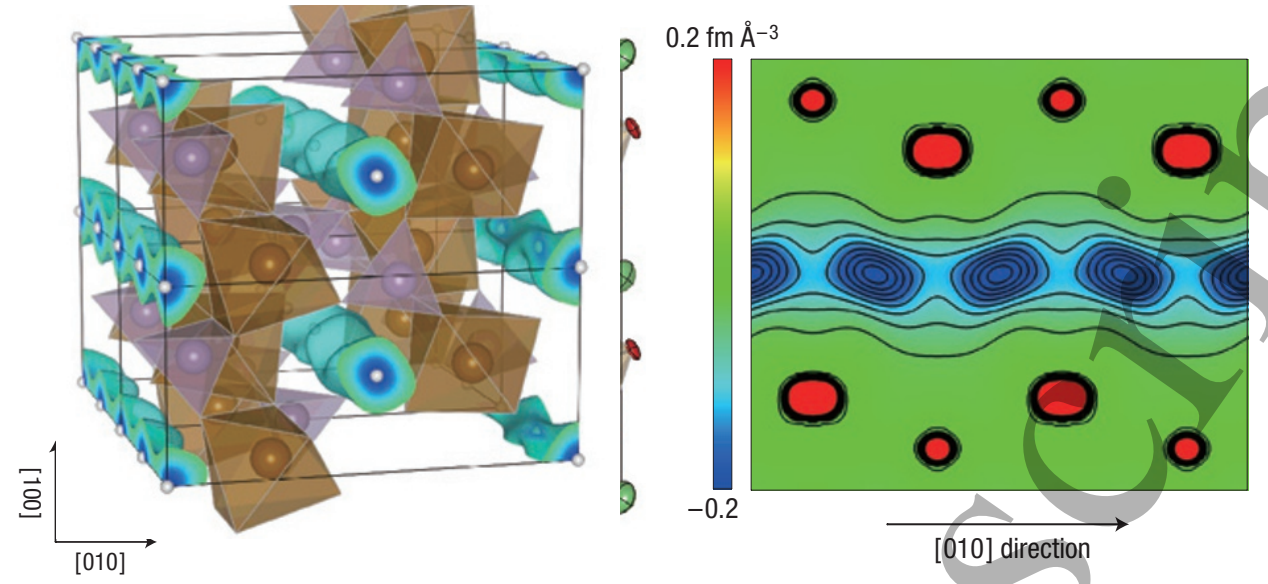

Figure 34: Anisotropic harmonic lithium vibration in $\mathrm{LiFePO}_{4}$. Expected curved onedimensional continuous chains of lithium motion are drawn as dashed lines to show how the motions of Li atoms evolve from vibrations to diffusion. Two-dimensional contour map sliced on the (001) plane at $\mathrm{z}=0.5$; lithium delocalises along the curved one-dimensional chain along the [010] direction, whereas Fe, $\mathrm{P}$, and $\mathrm{O}$ remain near their original positions. Adapted by permission from Springer Nature: Ref. 681, Copyright 2008.

2596

Chemical diffusion coefficient of $\mathrm{Li}$ in an intercalation compound often has a strong dependence on Li concentration and crystal structure. The combination of DFT cluster expansion Hamiltonians with kinetic Monte Carlo (kMC) simulations, as described in sections 2.1.4 and 2.1.5 revealed that the Li diffusion coefficients of TM oxides (and sulfides) are very sensitive to the Li concentration and also to the degree of cation ordering. $\frac{77682 \sqrt[685]{60 r}}{76}$ example, Van der Ven et al. shows the calculated Li diffusion coefficients for the layered (2D) and spinel (3D) forms of $\mathrm{Li}_{x} \mathrm{TiS}_{2}$ as a function of Li concentration. ${ }^{9|682| 683 \mid 685}$ This is presented in Figure 35, along with the structural images and vacancy mechanisms highlighted. Here it can be seen that not only do the Li diffusion coefficients differ by orders of magnitude, but the shape of the diffusion/Li concentration relation is very different. This shows how the crystal structure, and thus the active diffusion pathways, plays a crucial role in determining the concentration dependence of the diffusion coefficients in these materials.

We have already eluded that diffusion is sensitive to the Li-ion concentration. However, the exact relation is through the activation barriers. Early DFT studies ${ }^{\sqrt[678686]{6}}$ of $\mathrm{Li}_{x} \mathrm{CoO}_{2}$ 

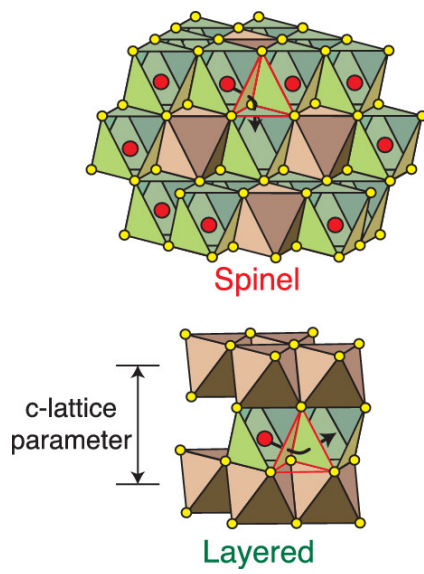

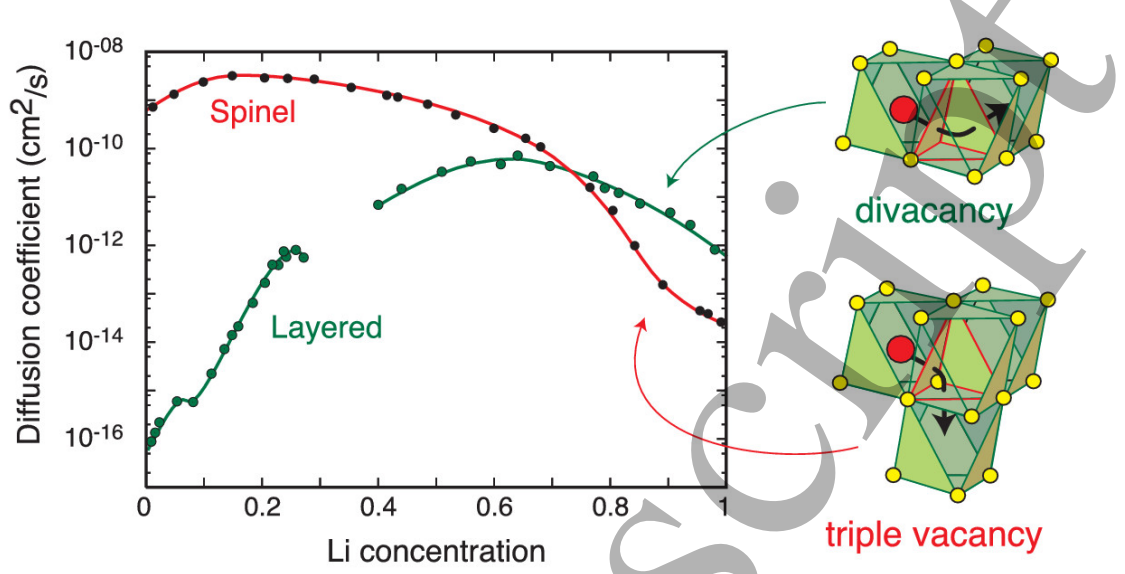

Figure 35: Chemical diffusion coefficient of Li in an intercalation compound often has a strong dependence on Li concentration and crystal structure. Reprinted with permission from Ref. 9. Copyright 2020 American Chemical Society.

2611

2612

2613

2614

2615

2616

2617

2618

2619

2620

2621

2622

2623

2624

2625

2626

nism, when $0 \leq x<1$. However, at infinite vacancy dilutions diffusion is through a single vacancy mechanism. $\frac{606}{6}$ There are two hopping mechanisms at play here; oxygen dumbbell hops and tetrahedral site hops. Oxygen dumbbell hopping occurs when there is a single vacancy and a Li-ion has to travel between two occupied adjacent lithium sites to reach the vacant lithium site. Tetrahedral site hopping occurs when there are divacant or trivacant sites, i.e. when one or both of the adjacent lithium sites are vacant. ${ }^{686}$ Oxygen dumbbell hopping has a significantly lower migration barrier energy compared to tetrahedral site hopping, which highlights the sensitivity of the activation barrier to the lithium concentration.

Experimental studies of mixed-TM layered oxides, such as $\mathrm{Li}\left(\mathrm{Ni}_{0.5} \mathrm{Mn}_{0.5}\right) \mathrm{O}_{2}$, have reported site exchange between Li and $\mathrm{Ni}(\sim 8-12 \%) .{ }^{687}$ DFT has been used to aid in understanding

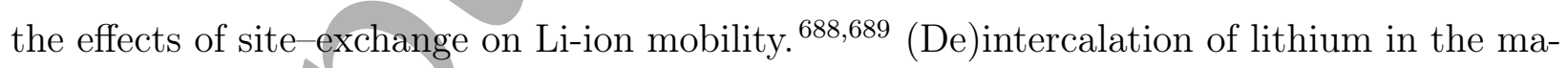
terial changes the distances between the layers. As Li is removed from the structure, there is a reduced "barrier" between the oxygen layers which start to repel one another. By calculating the activation energy as a function of the distance between the O layers on either side of the Li layers, a trend between increased O layer separation and lower activation energy is seen. 6881689 In addition to the crystal structure and available diffusion pathways, doping the cathode 
material can also influence the material properties, including ion diffusion. NMC cathodes are effectively $\mathrm{LiCoO}_{2}$ doped with $\mathrm{Ni}$ and $\mathrm{Mn}$. As previously mentioned in section 5.1 . introducing $\mathrm{Ni}$ and $\mathrm{Mn}$ into the system to form a mixed-TM layered oxide increases the diffusion/conductivity and electrochemical performance. There are very few detaiked computational studies of mixed-TM oxides due to their complexities. An illustration of this is the complexities which arise from TMs, such as $\mathrm{Fe}, \mathrm{Ni}, \mathrm{Co}$, and Mn, which exhibit localised oxidation states. This can be further complicated, or influenced by, TM ordering. For instance, Lee and Persson investigated the effects of TM disorder on the electrochemical properties of $\mathrm{Li}_{x} \mathrm{Ni}_{0.5} \mathrm{Mn}_{1.5} \mathrm{O}_{4}$ using cluster expansion and MC methods (c.f. sections 2.1.4 and 2.1.5). The authors determined a correlation between Li vacancy ordering and TM ordering. ${ }^{690}$ HaO et al. found similar evidence for $\mathrm{Li}_{x}\left(\mathrm{Mn}_{y} \mathrm{Ni}_{1-y}\right)_{2} \mathrm{O}_{4} \cdot \frac{691}{6}$ These also have an effect on the diffusion properties of the material. TM ordering in NMC cathodes is discussed in more detail in section 5.2.4. Using experimental techniques, Capsoni et al. found that doping the cationic sublattice of spinel $\mathrm{LiMn}_{2} \mathrm{O}_{4}$ with as low as $1 \%, \mathrm{Ga}^{3}+$ significantly modifies the temperature of the conductivity drop associated with Jahn-Teller (JT) distortion, preventing the transition observed near room temperature. ${ }^{692}$ This allows for a wider temperature window for the higher conductivity phase. DFT using generalised gradient approximation (GGA) or its variant $\mathrm{GGA}+\mathrm{U}$ (c.f. section 2.1.1), was also employed to analyse the effect of doping $\mathrm{LiMn}_{2} \mathrm{O}_{4}$ on the JT distortion. In this study, Singh et al. found that doping with $\mathrm{Cr}$ and $\mathrm{Mg}$ also suppressed the JT distortion and thus the associated temperature of the conductivity drop. .693

\subsubsection{Redox and Electronic Properties}

The cathode operates by the deintercalation of $\mathrm{Li}^{+}$on charging, and the reinsertion of $\mathrm{Li}^{+}$ on discharging. The charge is balanced by the oxidation and reduction of the TM ion, e.g. $\mathrm{LiCo}^{3+} \mathrm{O}_{2} \rightleftharpoons \mathrm{Li}_{1-x} \mathrm{Co}^{4+} \mathrm{O}_{2}+x \mathrm{Li}^{+}+x \mathrm{e}^{-}$. The role of TM redox in LiBs has been well known since the first publications by Goodenough on $\mathrm{LiCoO}_{2}$ as an intercalation electrode 
in 1980.585 Although various classes of compounds have been investigated over the years, the overall mechanism of TM redox is broadly similar. The three major classes of oxide cathodes, (layered, $\underline{585}$ polyanion, $\underline{694}$ and spinel ${ }^{695}$ ) all function via a TM redox couple. The specific capacity of most LiB cathode materials is limited by the number of electrons per TM cation that can participate in the redox reaction. However, the recent discovery of oxygen redox

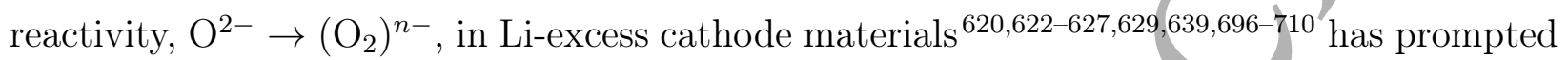
further investigation.

DFT has been pivotal in shedding light on this phenomenon, in conjunction with a range of experimental techniques. DFT can be used to analyse the atomic charge and electronic structure of each ground state, enabling the charge compensation during delithiation to be correctly attributed during simulated charging. Yao et al. were able to propose a sequence

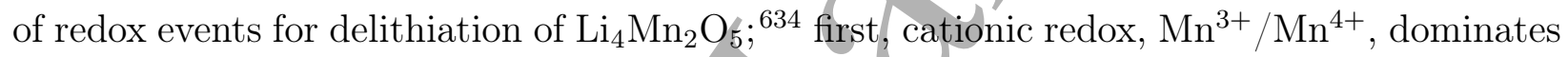
for $\mathrm{Li}_{x} \mathrm{Mn}_{2} \mathrm{O}_{5}$, when $4 \geq \mathrm{x}>2$. Then anionic redox, $\mathrm{O}^{2-} / \mathrm{O}^{1-}$, dominates for $\mathrm{Li}_{x} \mathrm{Mn}_{2} \mathrm{O}_{5}$, when $2 \geq \mathrm{x}>1$. Finally, mixed cationic $\left(\mathrm{Mn}^{4+} / \mathrm{Mn}^{5+}\right)$ and anionic $\left(\mathrm{O}^{2-} / \mathrm{O}^{1-}\right)$ redox for $\mathrm{Li}_{x} \mathrm{Mn}_{2} \mathrm{O}_{5}$, when $1 \geq \mathrm{x} \geq 0$. Meanwhile, fluorinated materials such as $\mathrm{Li}_{2} \mathrm{Mn}_{2 / 3} \mathrm{Nb}_{1 / 3} \mathrm{O}_{2} \mathrm{~F}^{649}$ and $\mathrm{Li}_{2} \mathrm{MnO}_{2} \mathrm{~F}^{639}$ were found to exhibit some overlap between the redox processes, suggesting that the substitution of $\mathrm{O}$ by $\mathrm{F}$ favours lower $\mathrm{Mn}$ oxidation states, therefore leading to more redox overlap with oxygen. DFT has also been used to establish the band structure for cathode materials, determining which TM orbitals hybridise more with the $\mathrm{O}(2 \mathrm{p})$ orbitals $\underline{6991711}$ and to identify hole states. $.704 / 712$

In a combined experimental and computational study, Gent et al. observed a strong correlation between anion redox, cation migration, and open circuit voltage (OCV) hysteresis in Li-rich layered oxides. ${ }^{624}$ Hong et al. offered an explanation for the strong coupling between anion redox and structural disordering in Li rich layered oxides; they found local stabilisation of short $\sim 1.8 \AA$ metal-oxygen $\pi$ bonds and $\sim 1.4 \AA$ O-O dimers during oxygen redox. $\frac{713}{7}$ Seo et al. showed that anion redox chemistry is heavily dependent on the anion nearest${ }_{2680}$ neighbour coordination environment. $\frac{623}{}$ In particular, they described how more Li-O-Li con- 
figurations lead to more potentially labile oxygen electrons, resulting in enhanced $\mathrm{O}$ redox chemistry, as shown in Figure 36, A similar result was found with $\mathrm{Li}_{2} \mathrm{MnO}_{2} \mathrm{~F}$; those oxygens coordinated to at least five $\mathrm{Li}\left(\mathrm{e} . \mathrm{g} . \mathrm{OLi}_{5} \mathrm{Mn}\right)$ in the fully lithiated state were the first to oxidise, whereas those coordinated to three or fewer (e.g. $\mathrm{OLi}_{3} \mathrm{Mn}_{3}$ ) did not undergo oxidation at all. This showcased a more continuous variation in the O-redox potential, dependent on the number of Li coordinated to a given $\mathrm{O}^{2-}$ ion. ${ }^{639}$ Recent computational screening work on layered oxide cathodes using hybrid DFT has reported trends in O-redox activity associated with the electrostatic (Madelung) energy at oxygen sites.

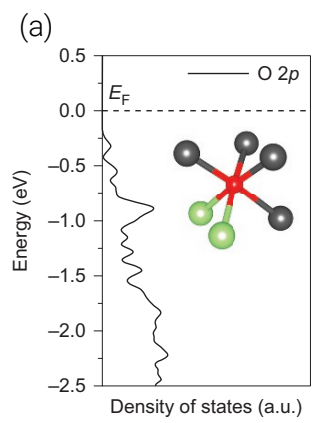

(e)

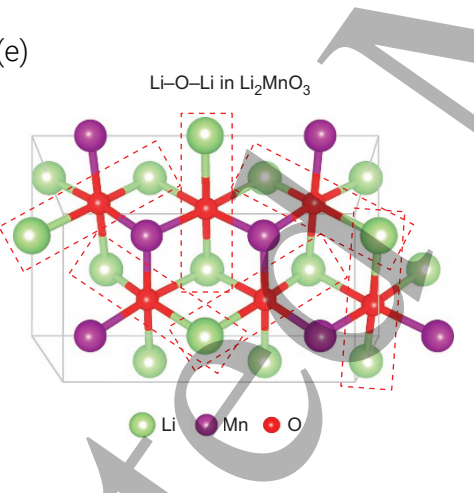

(b)

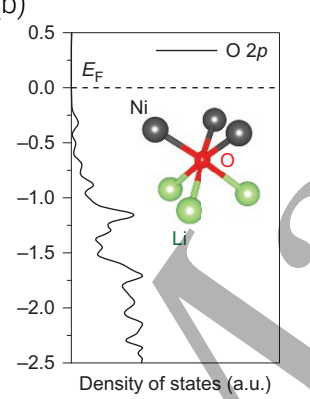

(c)

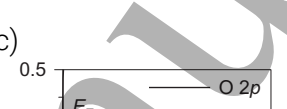

(d)

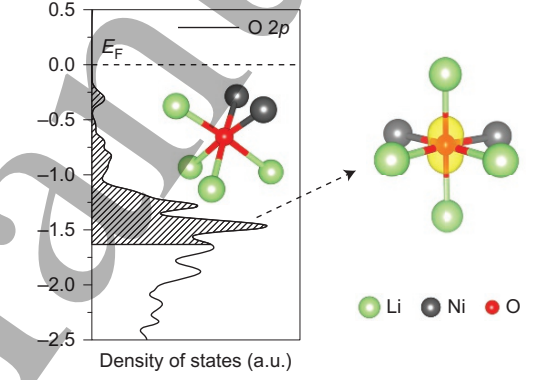

(g)
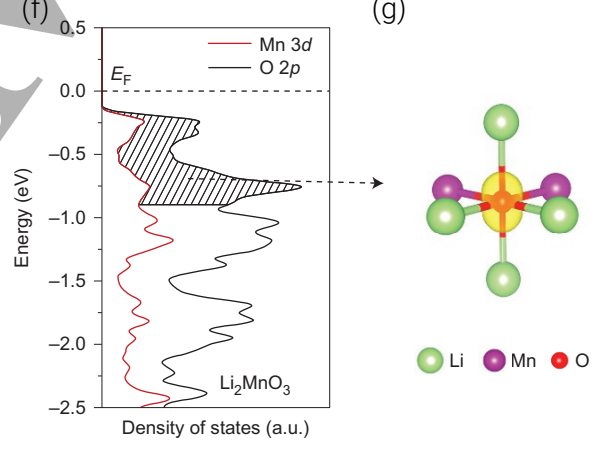

Figure 36: Effect of local atomic environments on the electronic states of $\mathrm{O}$ ions in (a-d) cation-mixed layered $\mathrm{LiNiO}_{2}$ and (e-g) $\mathrm{Li}_{2} \mathrm{MnO}_{3}$. Cation mixing introduces various local environments around oxygen. Projected density of states (pDOS) of the O $2 p$ orbitals of $\mathrm{O}$ atoms in cation-mixed layered $\mathrm{LiNiO}_{2}$ coordinated by (a) two $\mathrm{Li}$ and four $\mathrm{Ni}$, (b) three $\mathrm{Li}$ and three $\mathrm{Ni}$, and (c) four $\mathrm{Li}$ and two $\mathrm{Ni}$. (d) gives the isosurface of the charge density (yellow) around the oxygen coordinated by four $\mathrm{Li}$ and two $\mathrm{Ni}$, in the energy range of 0 to $-1.64 \mathrm{eV}$. (e) gives an illustration of Li-O-Li configurations in $\mathrm{Li}_{2} \mathrm{MnO}_{3}$, with (f) giving the related pDOS of the $\mathrm{O} 2 p$ orbitals and Mn $3 d$ orbitals, and (g) giving the isosurface of the charge density (yellow) around the oxygen, in the energy range of 0 to $-0.9 \mathrm{eV}$. Adapted by permission from Springer Nature: Ref. 623, Copyright 2016.

Chen and Islam investigated delithiation and kinetic processes in $\mathrm{Li}_{2} \mathrm{MnO}_{3}$ using hybrid 

DFT and found that Li extraction is charge-compensated by oxidation of the oxide anion, so that the overall delithiation reaction involves lattice oxygen loss. $\frac{701}{1}$ Localised holes on oxygen $\left(\mathrm{O}^{-}\right)$are formed at the first step but, due to their instability, lead to oxygen dimers (O-O is approximately $1.3 \AA$ ) and eventually to the formation of molecular $\mathrm{O}_{2}$. This then facilitates Mn migration to the octahedral site in the vacant Li layer, leading to a spinel-like structure. DFT has also been used to show the formation of $\mathrm{O}_{2}$ at high states of charge in $\mathrm{Li}_{2} \mathrm{MnO}_{2} \mathrm{~F}^{639}$ and $\mathrm{Li}_{1.2} \mathrm{Ni}_{0.13} \mathrm{Co}_{0.13} \mathrm{Mn}_{0.54} \mathrm{O}_{2},{ }^{628}$ agreeing with experimental resonant inelastic X-ray scattering (RIXS) data, and to report superoxide formation in $\mathrm{Li}_{2} \mathrm{VO}_{2} \mathrm{~F}$, in agreement with electron paramagnetic resonance (EPR) spectroscopy studies. ${ }^{648}$

\subsubsection{TM Ordering in NMC Layered Oxides}

Cation/anion ordering also plays a vital role in the properties/activity of a material, such as the physical and electrochemical properties. A topical illustration of this is the NMC cathode materials, where recent experimental studies show that spin interaction of the TM ions is a major challenge. $\frac{617 / 715}{6}$ The varying compositions, charge distributions, and electronegativities of the TMs lead to a mixture of valence states, where $\mathrm{Ni}$ can exist as $\mathrm{Ni}^{2+}$, $\mathrm{Ni}^{3+}$, and $\mathrm{Ni}^{4+}$, Co can exist as $\mathrm{Co}^{3+}$ and $\mathrm{Co}^{4+}$, and $\mathrm{Mn}$ exists as $\mathrm{Mn}^{4+} .715$ The interactions between these mixed valence states poses a challenge to the identification of ground states. As NMC materials, such as NMC811, emerge as front runners for commercialisation, research into their specific chemistry has become of great interest. Recently, several computational studies have been performed to analyse the influence of TM valence states on the stability and structure-property relationships of NMC materials, which are challenging to resolve experimentally. ${ }^{716}$ 719 For example, Sun and Zhao analysed 81 NMC compositions using DFT, observing that random arrangements of TMs present similar thermodynamic states. ${ }^{716}$ Clusters of random geometries and population were seen, which confirmed that no specific ordering exists at the superlattice scale. This is consistent with previous experi${ }_{2715}$ ment analysis using X-ray and neutron diffraction characterization on a specific composition 
2716

2717

2718

2719

2720

2721

2722

2723

2724

2725

2726

2727

2728

2729

2730

2731

2732

2733

2734

2735

2736

2737

2738

2739

2740

of $\mathrm{NMC}, \mathrm{Li}_{2 / 3}\left[\mathrm{Co}_{x} \mathrm{Ni}_{1 / 3-x} \mathrm{Mn}_{2 / 3}\right] \mathrm{O}_{2}$, demonstrating that Co suppresses the superlattice ordering when $x>1 / 6 . \sqrt[720]{n}$ The authors also demonstrated, through intensive computational screening, that no long-range ordering exists in the TM layer of NMC. These DFT studies provide fundamental understanding of the physicochemical properties at the intrinsic level of electronic structures and will offer important insight in the selection of NMC materials for enhanced electrochemical performance. It would not be tractable to analyse so many compositions of NMC through experiments.

\subsubsection{Vibrational and Thermal Properties}

An important contribution to the thermodynamic properties at finite temperature is the vibrational partition function, which can be evaluated by calculating the material's normal modes of lattice vibrations. A number of rêsearchers have theoretically addressed the vibrational contribution to the material thermodynamic properties in LiBs, especially in NMC cathodes. ${ }^{181|182| 721}$ There are several works studying cathode materials beyond NMC. Shang et al. employed DFT phonon calculations with a mixed-space approach to probe the lattice dynamics and finite-temperature thermodynamic properties of olivine structure $\mathrm{LiMPO}_{4}(\mathrm{M}$ $=\mathrm{Mo}, \mathrm{Fe}, \mathrm{Co}, \mathrm{Ni}) \cdot \stackrel{722}{ }$ The authors reported that $\mathrm{LiMPO}_{4}$ structures from $\mathrm{Mn}$, Fe, Co, to Ni show increasing zero-point yibrational energy, but a diminishing vibrational contribution to the Gibbs energy, due to the decreasing phonon densities of state at the low frequency region of $\mathrm{LiMPO}_{4}$. Recently, lattice dynamics studies have been expanded to solid electrolytes, aiding in the discovery of lithium fast-ion conductors. $\frac{723}{72}$

Two major approaches have been developed to compute lattice thermal conductivity; by solving the Boltzmann transport equation (BTE) using anharmonic lattice dynamics and through MD simulations. Puligheddu et al. compared lattice thermal conductivity values from these two methods and found a satisfactory agreement. ${ }^{724}$ The comparison used empirical potentials and took into account the effects of both fourth order phonon scattering ${ }_{2741}$ and temperature-dependent phonon frequencies, reporting the different effects of quantum 
and classical statistics.

Using BTE within the relaxation-time approximation, Mattila and Karttunen reported the highly anisotropic lattice thermal conductivities in isotopic $\mathrm{LiCoO}_{2}$, close to the values in Yang et al.'s work, $\frac{181 \mid 182}{182}$ and illustrated the effect of the alkali metal atom by replacing Li by $\mathrm{Na} \cdot \stackrel{725}{7}$ The authors explained this through the significantly shorter phonon lifetimes in $\mathrm{LiCoO}_{2}$. They found that in-plane lattice thermal conductivities in $\mathrm{NaCoO}_{2}$ are $\sim 0.7$ times larger than that in $\mathrm{LiCoO}_{2}$ at room temperature, since the former has significantly longer phonon life times. While Feng et al. report much lower thermal conductivity values by including four-phonon scattering, using a different functional, the local density approximation (LDA), for exchange and correlation. $\frac{726}{}$ They also investigated the thermal transport reduction during delithiation (charging) due to reduced phonon velocities and increasing anharmonicity. Furthermore, grain-boundary'effects reduced thermal transport and suppressed thermal conductivites in polycrystals are well reproduced when grain sizes were reduced down to several $\mathrm{nm}$ in either BTE or MD simulations. 155

The thermal conductivity investigation can be also performed on anodes and many other materials. ${ }^{727 / 728}$ Recently, a high-throughput study was reported for 37 binary rock-salt and zinc blende material systems, in which the authors highlight the importance of high-order phonon-phonon interactions based on harmonic calculations. ${ }^{[729}$ Modelling heat transport using DFT calculations is complex but essential due to the difficulties inherent in preparing high-quality samples for experimental measurements.

\subsection{Surfaces}

Surface structures and morphologies of cathode particles can be difficult to determine using experimental microscopy and spectroscopy methods alone and thus computational investigations can provide vital insights. ${ }^{730}$ Due to their synthesis conditions, experimental cathode materials comprise different surface facets, defects, and particle sizes. It is therefore necessary to use model systems to determine which of these effects is more important by studying 
them in isolation, separating the effects, which is not possible using experimental materials. Both ab initio and potentials-based MD have been extensively used to investigate the surfaces and morphologies of layered oxides, spinel oxides, and olivine phosphates, which will be briefly discussed here. These techniques have also been used to investigate/cathode materials in sodium-ion batteries, which is covered in more detail in Ref. 606

With oxides at the forefront of the battery revolution, it is unsurprising that there have been many DFT and potentials-based MD studies into layered $\mathrm{LiCoO}_{2}, \mathrm{LiMn}_{2} \mathrm{O}_{4}$ spinel, $\mathrm{MnO}_{2}$-type and related materials, looking at properties including the surfaces, nanostructures, and morphologies. ${ }^{171}$ S38 Surface energies for low-index layered $\mathrm{LiCoO}_{2}$ surfaces, as a function of external $\mathrm{Li}$ and $\mathrm{O}$ chemical potentials, revealed the (0001) and (1014) surfaces were present for all reasonable values of $\mathrm{Li}$ and $\mathrm{O}$ chemical potentials, whereas the $(01 \overline{1} 2)$ surface was only stable under oxidising conditions. ${ }^{731}$ Studies into the low-index surface facets of $\mathrm{LiMn}_{2} \mathrm{O}_{4}$ determine the (111) surface to be the most stable. This is due to the site exchange of under-coordinated Mn on the surface, which exhibit a cubo-octahedral type, predominately comprising $\{111\}$ surfaces. ${ }^{736}$ Other studies show that the Mn-terminated (111) surfaces undergo surface reconstruction, indicating instead that the Li-terminated (001) surface has the lowest energy.

It has also been shown that electronic spin state transitions occur on the surfaces of stoichiometric $\mathrm{LiCoO}_{2}$. Here Qian et al. found that the trivalent Co ions at the surface adopt an intermediate spin state if they are square-pyramidally coordinated and a high spin state if they are pseudo-tetrahedrally coordinated. This highlighted the effect of low-coordinated geometries at the particle surface on the $\mathrm{Co}^{3+}-\mathrm{Co}^{4+}$ redox potential. ${ }^{739}$ Hong et al. investigated the surface properties of $\mathrm{LiCoO}_{2}$ nanoplatelets and their chemical modifications with $\mathrm{Al}^{3+}$, using combined experimental and theoretical approaches. ${ }^{740}$ Their models also showed the electronic structures of several $\mathrm{LiCoO}_{2}$ surface facets are different from those of the bulk, attributing this to the altered spin states of surface $\mathrm{Co}^{3+}$ atoms. The authors found splitting ${ }_{2794}$ of the Co $3 \mathrm{~d}-\mathrm{O} 2 \mathrm{p}$ states, which were linked with high-spin-state $\mathrm{Co}^{3+}$ at the surface. Partial 
substitution of $\mathrm{Co}^{3+}$ by $\mathrm{Al}^{3+}$ was found to increase the ratio of low-spin-state $\mathrm{Co}^{3+}$ at the surface, resulting in a distinct change in the intensity ratio of the split Co $3 \mathrm{~d}-\mathrm{O} 2 \mathrm{p}$ states.

When exposed to certain environmental conditions, $\mathrm{LiCoO}_{2}$ releases $\mathrm{Co}$ cations, a known toxicant. Abbaspour-Tamijani et al. has applied DFT (with different functionals) and thermodynamics modelling to study the $\mathrm{LiCoO}_{2}$ surface transformations. ${ }^{741}$ They assessed how the calculated predictions for ion release depend on aspects of the structural surface model. Here, the authors propose a generalised scheme for predicting a threshold $\mathrm{pH}$ at which Co release becomes favourable, providing information that could be used to inform macroscopic contaminant fate models. More recently, these authors have furthered this investigation in cation dissolution at the $\mathrm{LiCoO}_{2}$ surface, finding that at a $\mathrm{pH}$ of $7,16 \%$ of surface $\mathrm{Co}$ undergoes dissolution.

Phase transitions in cathode materials can have negative effects on the desirable properties. However, there are circumstances where use of different structural phases are beneficial. For example, post-modification of Li-rich layered material surfaces to form a spinel $\mathrm{LiMn}_{2} \mathrm{O}_{4}$ membrane, i.e. encapsulating the layered particle, has shown enhanced related rate capability and cycling stability. $\frac{148 / 742743}{74}$ More significantly, insertion of a spinel component $\frac{744}{17}$ or the formation of platelets ${ }^{745}$ on layered-layered composites of NMC cathodes, yields a high specific capacity $\left(\sim 250 \mathrm{mAh}^{-1}\right)$ and can partly correct for voltage fade. $\stackrel{148}{ }$ Phase transitions can also be a negative consequence of particle surface stress. Warburton et al. investigated the particle fracturing in $\mathrm{LiMn}_{2} \mathrm{O}_{4}$ caused by stress through the delithiation process. $\frac{[746}{{ }^{-1}} \mathrm{Using}$ DFT, the authors provide a good understanding of the stress buildup at the surface during delithiation, demonstrating that the delithiation of near-surface layers contribute towards the buildup, leading to a $\mathrm{LiMn}_{2} \mathrm{O}_{4} / \mathrm{Li}_{0.5} \mathrm{Mn}_{2} \mathrm{O}_{4}$ low-voltage phase transition, Figure 37. The authors also investigate if there is an orientation preference, concluding that cracks due to tensilestress buildup are not likely to orient preferentially in the [001] direction, because the stresses act in the plane of the (001) surface. ${ }^{746}$ This shows that an in-depth understanding of 2821 the electrochemical processes of cathode materials, at the atomistic scale, is urgently needed, 
especially for more complex chemistries like NMC. A recent study on the NMC surfaces by Liang et al. looked at the surface segregation and anisotropy using DFT $+\mathrm{U}$ calculations. $\frac{747}{7}$ In this study, the authors looked at surface stability, morphology, and elastic anisotropy, all related to the degradation of Li-ion batteries. Ni surface segregation predominantly occurs on the (100), (110), and (104) nonpolar surfaces, showing a tendency to form a rock-salt $\mathrm{NiO}$ domain on the surface, due to severe Li-Ni exchange. The findings of this study showed that an uneven deformation is more likely to form in particles which have been synthesised under low oxygen conditions, leading to crack generation and propagation. 747
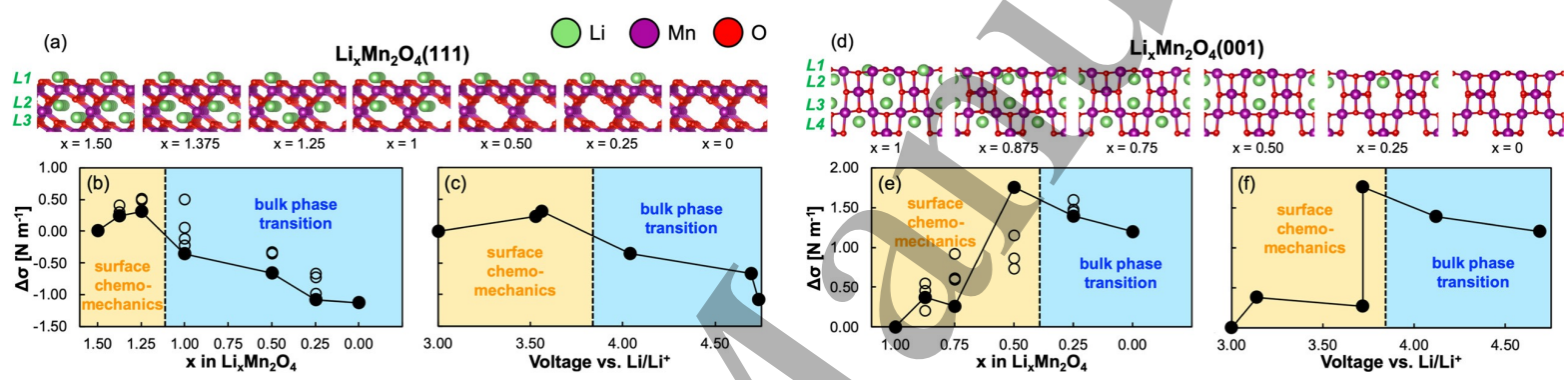

Figure 37: Surface stress evolution upon delithiation of lithium manganese oxide (LMO) surfaces. Low energy structures of the (a) LMO(111) and (d) LMO(001) surfaces at different $\mathrm{Li}^{+}$contents. Differential surface stresses of (b) LMO(111) and (e) LMO(001) as a function of the $\mathrm{Li}^{+}$content for various $\mathrm{Li}^{+}$configurations. The filled circles in (b,e) represent the most energetically stable structures for a given stoichiometry. The unfilled circles in (b,e) denote metastable lithium configurations. Differential surface stresses of (c) LMO(111) and (f) $\mathrm{LMO}(001)$ as a function of the cell voltage. The dashed lines correspond to the calculated equilibrium potential of $3.84 \mathrm{~V}$ vs $\mathrm{Li} / \mathrm{Li}^{+}$between $\mathrm{LMO}$ and $\mathrm{L}_{0.5} \mathrm{MO}$. The yellow-shaded regions correspond to surface-dominated mechanics from the near-surface delithiation. The blue-shaded regions correspond to surface phases that are thermodynamically inaccessible because they become stable only at voltages above the equilibrium potential. Reprinted with permission from Ref 746. Copyright 2020 American Chemical Society.

The surface structures of $\mathrm{LiFePO}_{4}$ exhibit a complex and uneven topology due to the size difference of $\mathrm{Li}^{+}, \mathrm{Fe}^{2+}$, and $\mathrm{PO}_{4}^{3-}$. The majority of terminating surfaces undergo fairly considerable relaxation, which makes predictions based on rigid terminations unreliable. Although $\mathrm{LiFePO}_{4}$ can be synthesised in multiple morphologies exposing different surfaces, ${ }^{6667748}$ studies on the (010) surfaces are particularly interesting. This surface is nor- 

lengths for lithium at the surface, enhancing the electrochemical performance of the cathode. DFT calculation of the diffusion pattern and energy landscape of lithium in $\mathrm{LiFePO}_{4}$ showed that the energy barrier for the Li diffusion along (010) is lower than along the other directions, e.g. (100), indicating that the $\mathrm{Li}$ diffusion in $\mathrm{LiFePO}_{4}$ is one dimensional. 750 Understanding processes such as the lithium (de)intercalation on the $\mathrm{LiFePO}_{4}(010)$ surface is important for developing effective approaches for further improving the material's rate performance. Using DFT calculations, $\mathrm{Xu}$ et al. found that the extraction of Li from the surface layer has a significant effect on the work function of the $\mathrm{LiFePO}_{4}(010)$ surface, providing evidence for whether Li atoms are present in the/outermost layer of $\mathrm{LiFePO}_{4}(010)$ surface or not. ${ }^{751}$ Here, the authors also calculate the redox potential and formation energies for extracting Li from different (010) surface layers. They find that extracting lithium from the outer surface layers has the lowest redox potential and formation energy, indicating that it is energetically favorable to extract Li first from the surface layer. $\mathrm{Xu}$ et al. propose a new method that surface work functions can be used for providing insight into the lithium (de)intercalation on the $\mathrm{LiFePO}_{4}(010)$ surface. $\frac{751}{}$

Zhang et al. used a combined experimental and computational (DFT) approach to investigate the preferential cation doping on the surface of $\mathrm{LiFePO}_{4}$ and its effect on properties. $\frac{752}{72}$ The authors found that, for all chosen dopants, there were increased ratios of $\mathrm{Fe}^{3+} / \mathrm{Fe}^{2+}$ oxidation on the particle surfaces, while the core atoms remained closer to that of the pristine, undoped material. This indicates that the dopants are predominantly pushed to the particle surfaces during phase formation. This disparity in distribution of dopant across the core and surface results in improved conductivities. ${ }^{752}$ ab initio MD simulations with X-ray Diffraction (XRD) and microscopy experiments on the $\mathrm{LiFePO}_{4}$ cathode show Li-ions migrating along the surface, facilitated by solvent molecules. $\frac{753}{75}$ This work establishes fluid-enhanced surface diffusion as a key factor in tuning phase transformation in anisotropic solids. 
2861

\subsection{Interfaces}

Although the cathode-electrolyte interphase (CEI) is thinner than the SEI at the anode, it is still quite complex in structure and composition.115/754 DFT-based simulations can provide insight into adsorption trends, $\frac{755}{r}$ reaction pathways and energetics, $\stackrel{756 / 757 /}{1}$ and migration barriers for Li-ion transfer, ${ }^{758}$ etc. The electrolyte in a Li-ion battery is typically a Li salt, for example $\mathrm{LiPF}_{6}$ in an organic carbonate solvent, such as ethylene carbonate (EC), propylene carbonate (PC), diethyl carbonate (DEC) or dimethyl carbonate (DMC). The $\mathrm{LiPF}_{6}$ electrolyte reacts with trace amounts of moisture to form hydrofluoric acid (HF), which is highly corrosive and reacts with the cathode surface to form fluoride-based products. $\frac{757}{7}$ The organic carbonate solvent also reacts with the cathode surface to form a series of decomposition products. $\frac{759}{75}$ The adsorption of solvent-décomposition and fluoride-based products is the first step in the series of reactions that lead to the formation of the CEI. The decomposition reaction of cyclic organic carbonate solvents proceeds via ring opening, having an energy barrier predicted via climbing image nudged elastic band (CI-NEB) calculations (sections 2.1.1 and 2.1.3 to be around $0.62 \mathrm{eV}$ on (100) $\mathrm{LiMn}_{2} \mathrm{O}_{4}$ surfaces, $\stackrel{737}{ }$ over $1 \mathrm{eV}$ on $(101 \overline{4}) \mathrm{LiCoO}_{2}$ surfaces, ${ }^{759}$ and around $0.29 \mathrm{eV}$ on $(101 \overline{4}) \mathrm{Li}(\mathrm{Ni}, \mathrm{Mn}, \mathrm{Co}) \mathrm{O}_{2}$ surfaces. ${ }^{760}$ While experimental studies on the composition of the CEI have shown the presence of both solvent-decomposition and fluoride-based products on most oxide cathodes, such as $\mathrm{LiMn}_{2} \mathrm{O}_{4}, \mathrm{LiNiO}_{2} \mathrm{LiCoO}_{2}$ and $\mathrm{LiNi}_{0.8} \mathrm{Co}_{0.2} \mathrm{O}_{2}$, no solvent reaction or solvent decomposition products are detected on $\mathrm{LiFePO}_{4} \cdot{ }^{754 / 761}$ Recent calculations of adsorption energies based on DFT have shown that adsorption preference of $\mathrm{HF}$ over EC leads to the entire $\mathrm{LiFePO}_{4}$ nano-particle being covered/by fluoride-based products, further leading to their dominant presence in the CEI. ${ }^{755}$ DFT simulations have also been used to design suitable coatings in order to prevent cathode degradation. $\frac{757}{76}$ These calculations can shortlist effective candidate materials to guide experiments. Thus, atomistic methods can not only provide the necessary insights needed in order to explain experimental observations, but also suggest novel solutions for mitigating cathode degradation. 
Apart from the complexity of structure of the CEI, another challenge is understanding Li-ion migration at the CEI, impacting the rate capability of LiBs. Li-ion conductivity in bulk electrolyte is around $1 \mathrm{~S} \mathrm{~cm}^{-1}$ (c.f. section 4 which is several orders of magnitude higher than that in bulk electrode materials (c.f. sections 3.2 .4 and 5.2.2) (arourd $10^{-7}$ $\left.10^{-2} \mathrm{~S} \mathrm{~cm}^{-1}\right) \cdot \stackrel{287685}{2}$ However, the complex structure of the CEI and uncertainty about the mechanism of Li-ion transfer across it has hindered the understanding of kinetics at the interface. Recent NEB calculations on the $\mathrm{LiFePO}_{4}$ cathode have estimated an energy barrier of $756 \mathrm{meV}$, for Li to move from a near-surface solvated cluster to a sub-surface vacancy in the $\mathrm{LiFePO}_{4}$ cathode material. ${ }^{758}$ Due to preferential adsorption of fluoride on $\mathrm{LiFePO}_{4}$ surfaces, $\frac{754755}{7}$ the energy barrier has been found to decrease to $410 \mathrm{meV}$ in the presence of fluoride. Nevertheless, the interfacial energy barrier is higher than that in bulk cathode material, which is estimated to be around $270-290 \mathrm{meV}$ 674/762 This highlights a rate-limiting behaviour of the interface in the overall Li-ion diffusion process in LiBs. This study motivates further investigation on other cathode electrolyte interfaces, especially with recently developed advanced methods for characterising the interface, as described in section 2.2.1.

\subsection{Outlook and challenges for cathodes}

Lowering the cost, increasing capacity, and improving the sustainability of battery materials is becoming more critical, as we move towards large-scale deployment of LiBs for applications such as EVs. ${ }^{588}$ Here, we highlight some of the outstanding challenges for cathodes and how atomistic modelling can provide insights and suggest solutions.

Ni-rich NMC layered oxides are favorite candidates for cathode materials, due to their high gravimetric and volumetric energy densities. ${ }^{763}$ However, these materials have three critical challenges: cycle instability, thermal instability, and air instability. These are all linked with the instability of $\mathrm{Ni}^{3+}$ and $\mathrm{Ni}^{4+}$ at the surface/interface. Other cathode materials, such as oxyfluorides, have worked towards solving some of these issues, however, there are still outstanding surface and interfacial challenges, for which atomistic modelling is vital: 
- In $\mathrm{Ni}$-rich $\mathrm{NMC}$, the unstable $\mathrm{Ni}^{3+}$ and $\mathrm{Ni}^{4+}$ react aggressively with the electrolyte to form thick CEI layers and cause Ni and Mn dissolution. The dissolute TMs then migrate to the anode and cause electrolyte decomposition, leading to thíck SEI layers which limit the battery cyclability. ${ }^{764765} \mathrm{CEI}$ and SEI formation are crucial challenges to be overcome for both conventional and solid-state batteries. Although electrochemical spectroscopic techniques have been used to obtain molecular scale information, further detail, which cannot be resolved using current experimental techniques, is needed to gain more reliable information. ${ }^{[766}$ For example, deconvoluting impedance components in two-terminal electrochemical impedance spectroscopy (EIS) data for materials that have similar time constants, such as solid-state lithium charge transfer in a cell with a graphitized carbon anode and $\mathrm{LiCoO}_{2}$ cathode, is challenging. ${ }^{767}$ Half-cell measurements can be used to study the/impedance of the two electrodes separately, but these measurements do not fully reflect the processes occurring in a full-cell battery at different states of (dis)charge. .768 Three-electrode cell configurations present a way to potentially disentangle the impedance components from the anode and cathode. .767 However, these measurements are franght with uncertainties, as the insertion of the reference electrode can fundamentally change the electrochemistry. ${ }^{7691770}$ This is where atomistic modelling is well suited to provide the fundamental understanding of the limiting rate constants in electrochemistry, that can be used to guide further experiments. As available experimental techniques are unable to provide significant insight into the atomistic mechanism of Li-ion transfer at the cathode-electrolyte interface, atomistic modelling is ideally suited to shed light in this area. For example, Bhandari et al. used DFT to investigate the interfacial Li-ion transfer mechanism at an atomic level, from bulk ethylene carbonate $(\mathrm{EC}) / \mathrm{LiPF}_{6}$ electrolyte into a $\mathrm{LiFePO}_{4}$ cathode, and provide an estimate on the corresponding energy barrier. ${ }^{758}$

- Phase transitions at the surface of cathode materials occur at a high state of charge and affect the surface reactivity, resulting in increased TM dissolution and CEI/SEI 
formation. The effect of this is rapid capacity fading during cycling. $\frac{771}{}$ Co-free Lirich layered oxides, such as $\mathrm{Li}\left[\mathrm{Li}_{0.2} \mathrm{Mn}_{0.6} \mathrm{Ni}_{0.2}\right] \mathrm{O}_{2}$, are appealing due to their low cost and high capacities $\left(300 \mathrm{mAh} \mathrm{g}^{-1}\right) \cdot[772773$ However, these materials undergo layered to spinel transitions due to low octahedral site stability of $\mathrm{Mn}^{3+}$, leading to voltage decay during cycling and Mn dissolution, ${ }^{[744}$ making these materials challenging to employ as a practical cathode. Atomistic insight into the mechanisms involved in these phase transitions, gained through $a b$ initio and potentials-based MD methods, can provide the detail and understanding needed to prevent these phase transitions from occurring.

- Some cathode materials show reversible O-redox, with lower voltage hysteresis and, where $\mathrm{O}_{2}$ is formed, it reincorporates into the lattice. ${ }^{639} \mathrm{In}$ contrast, other materials show irreversible O-redox, with $\mathrm{O}_{2}$ lost from the surface, 6281701775 leading to unwanted side reactions with the electrolyte. The formation and potential loss of molecular $\mathrm{O}_{2}$ is likely to be heavily dependent on local structure. In the case of $\mathrm{Li}_{2} \mathrm{MnO}_{2} \mathrm{~F}$, DFT showed that $\mathrm{O}_{2}$ is formed only in $\mathrm{O}-\mathrm{Li}$ rich areas, not in O-Mn rich areas. ${ }^{639}$ Meanwhile, other oxyfluorides, such as $\mathrm{Li}_{2} \mathrm{VO}_{2} \mathrm{~F}$, do not show molecular $\mathrm{O}_{2}$ formation at all, but instead form superoxides on charging. 648

It is challenging to model disordered systems as, by their very nature, they can have an almost infinite arrangement of atoms. Use of computational techniques, such as cluster expansion, to generate low energy structures of disordered rock-salts, is a promising route to more realistic DET studies. ${ }^{641}$

As discussed in sections 2.2.2 and 4.2.4 more careful considerations of the factors/parameters to include when fitting interatomic potentials for a system is key to improving the quality of research conducted through potentials-based modelling. It is commonplace to reuse potentials from literature sources, without determining how they were fitted, which can lead to inaccuracies in the calculations performed. For example, if the potentials for a cathode material were fitted only to lattice parameters, elastic constants, and the bulk modulus, then the potential would not be accurately representative of the cathode redox properties. If prop- 

erties such as the dielectric constant were included, then redox chemistry would be better represented. In effect, interatomic potentials in literature are not necessarily transferable to different types of study. It is not feasible to fit to every material property, however, a broader range of properties, most relevant to the study being conducted, is required. There are tools in development ${ }^{142|143| 145 \mid 164}$ aiming to make this potential fitting process more accessible to atomistic modellers, with the ability to fit to a larger range of parameters. However, there is still a need for improved transparency in the publication of studies using interatomic potentials. Use of machine learning to develop potentials has also shown to be a promising avenue. Deringer et al. recently published a progress update, showing how machine learning is improving interatomic potentials by "learning" from electronic-structure data, giving increased accuracy in approximating material properties. ${ }^{776}$

In-depth insight into the elemental distribution, electronic structure, and crystalline structure under electrochemical conditions is challenging to achieve experimentally. Atomistic techniques, including DFT and MD, are well suited to provide the insight needed for these properties. However, future research and development of cathode materials will require collaborative efforts, involving the disciplines of chemistry, physics, material science, nanoscience/nanotechnology, and computational modelling/simulation. 116

\section{Outlook}

In this review, we have introduced the key methods deployed in battery modelling at the atomistic scale (section 2.1) in lithium-ion batteries (LiBs) and solid-state batteries (ASSBs), which are collectively called lithium batteries. We have summarised progress in this field, covering models for anodes, liquid and solid electrolytes, and cathodes. Outlooks specific to these components are summarised as follows: anodes, in section 3.5, liquid electrolytes, in section 4.2.10, solid electrolytes, in section 4.3.5, and cathodes, in section 5.5 .

There are several notable developments in atomistic methods for lithium batteries which 
need to be addressed. These include development of a semi-grand canonical framework, incorporating order parameters, with initial promising work developed by Van der Ven et al. 9,231

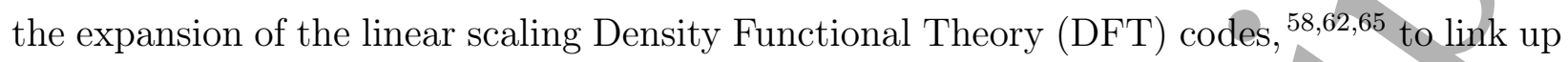
with kinetic Monte Carlo (kMC), the inclusion of entropy effects by parameterising a phase field model (such as those developed by Bazant) ${ }^{95}$ using results obtained from Monte Carlo (MC) calculations, development of more accurate force field potentials, and parallelisation to speed up MC calculations on longer length scales.

Alongside deepening our understanding of atomic structure and processes, atomistic models can be used to aid the design of new materials with improved capacity, rate capability, and stability. Atomistic modelling approaches have been shown to be strong tools to develop novel nanostructures and composites, understand dynamics and phase behaviour, and could identify novel interfaces to accommodate volume expansion in solid solution materials, such as silicides. Promising areas for future work include tuning the morphology and composition of graphite edges $\frac{70 \mid 254 \sqrt{255}}{\text { and interlayer spacings }}{ }^{214}$ to aid intercalation, improved understanding of the phase behaviour and dynamics of silicides as anode materials, $\stackrel{272}{21}$ and investigation of the emerging class of Li-rich cathode materials. $\underline{626|627| 629}$

We have identified several outstanding challenges for further work. For example, certain anode and cathode materials show pronounced hysteresis between charge and discharge cycles. $\frac{71|226| 272 \mid 777+779}{71}$ This results in a difference between expected equilibrium potentials from atomic-scale calculations and the experimentally measurable open circuit voltage (OCV), which creates ambiguity when using the measured OCV in longer length scale models, like control models for battery management systems. Future kinetic models must therefore account for metastable behaviour that can persist over experimental time scales of hours or even days. ${ }^{226}$ The next generation of models should consider the connectivity between different phase transformations, with the framework developed by Van der Ven et al. highlighted above being one promising solution that is potentially transferable to a variety of material 
Flammable liquid electrolyte materials currently dominate the commercial market. Development of safer, non-flammable, electrolyte materials is key to addressing safety concerns and accidents resulting from attempts to confine increasing energy densities into smaller volumes and into geometries that are challenging to thermally manage. More work is needed to investigate potential avenues for resolving these issues, including alternative liquid elec-

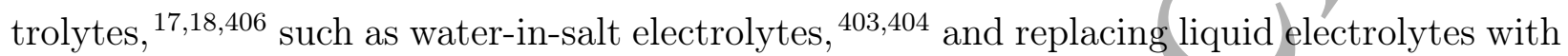
solid or soft matter alternatives. ${ }^{23567}$ Advancements in electrolyte design are crucial, where critical obstacles could be resolved through new novel electrolyte salts and solvents. Development and open source accessibility of atomistic scale models, combined with improved experimental studies, provide a framework for high throughput screening of electrolyte materials. $3571371 / 401402$

More work is needed to incorporate hetêrogeneities formed during material synthesis and

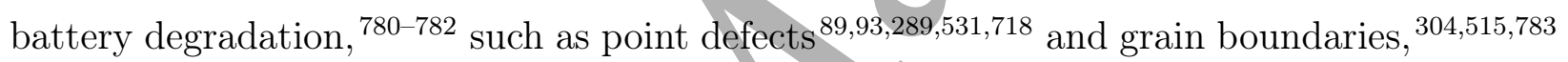
into atomistic models and to determine their effect on battery performance. Modelling of the complex behaviour at interfaces, such as the solid-electrolyte interphase (SEI) in LiBs and lattice mismatch in ASSBs, is a prominent challenge which requires further investigation. Atomistic models have already provided insight into particular aspects of degradation, leading to design of more robust materials, but the development of a universal framework for simulating degradation mechanisms and their interactions would be of great benefit and is still beyond current capabilities. In order for such a framework to be truly multiscale, significant work is needed to connect the modelling scales, linking atomistic to continuum modelling and on to longer length scales, such as control models, as well as forming closer links with experiments at all scales.

As we have seen throughout this review, different atomistic modelling approaches can be used individually to gain insight into different aspects and properties of LiB materials, across the wide range of time and length scales encompassing atomistic modelling. When combined into multiscale modelling (MSM) approaches, these techniques can provide a more complete 
interpretation of the material(s). ${ }^{10111233}$ A popular approach has been to combine DFT calculations of activation energies of different events, which are then used to implement kMC simulations. $\frac{104}{104}$ DFT calculations of the migration mechanisms and activation barriers of Liions have also been combined with classical MD studies of Li-ion diffusion to gain a more complete analysis of the dynamic properties in LiB materials. ${ }^{784785}$ Quantum, mechanical techniques, such as DFT, are also increasing being used to parameterise larger scale techniques, for example classical MD.14314511647776 The key consideration when designing MSM approaches is to reproduce the phenomena that dictate the natural behaviour and evolution of the material in given conditions. Thorough reviews, which focus more on the MSM aspect of $\mathrm{LiB}$ research, have recently been published by Franco et al. ${ }^{233}$ and Shi et al. ${ }^{10}$ kMC in particular has been highlighted as a natural technique to bridge length scales, naturally including different time scale dynamic events. ${ }^{104}$ In this review, we have instead focused on the individual contributions of atomistic techniques, the understanding of which is key to building MSM approaches. However, the full complexity of composite materials, such as $\mathrm{C} / \mathrm{Si}$, may only be accessible by bridging atomistic techniques to MSM such as via phase field methods, volume-averaged approaches, or mesostructurally-resolved models. 10111952233

This review has focused almost entirely on lithium batteries, given that they currently comprise the most technologically advanced rechargeable battery systems that are commercialised at scale. However, atomistic modelling applied to LiBs also improves understanding of batteries that could be based on more environmentally-friendly or Earth-abundant materials, such as sodium. Solid state models of intercalation, applied to LiBs, are directly transferable to other intercalation chemistries. The understanding of interfaces in batteries with other chemistries is even less developed than in LiBs. However, the modelling frameworks highlighted in this review, such as the linear-scaling DFT framework, could also be applied to improve understanding of these interfaces. 
30т2 Acknowledgement

3073

3074

3075

3076

3077

3078

3079

3080

3081

3082

3083

3084

3085

3086

3087

3088

3089

3090

3091

3092

3093

The authors thank the Faraday Institution (https://faraday.ac.uk/; EP/S003053/1), grant number FIRG003, for funding, and Dr. Maxim Zyskin for his discussions. We also thank Mr. Amir Kosha Amiri for his graphical design expertise in constructing and formatting the figures, as well as Dr. Felix Hanke and Dr. Victor Milman from BIOVIA for their comments and suggestions.

\section{References}

(1) Zeng, X.; Li, M.; Abd El-Hady, D.; Alshitari, W.; Al-Bogami, A. S.; Lu, J.; Amine, K. Commercialization of lithium battery technologies for electric vehicles. Advanced Energy Materials 2019, 9, 1900161.

(2) Goodenough, J. B.; Kim, Y. Challenges for rechargeable Li batteries. Chemistry of Materials 2010, 22, 587-603.

(3) Kubiak, P.; Cen, Z.; López, C. M.; Belharouak, I. Calendar aging of a 250 kW/500 kWh Li-ion battery deployed for the grid storage application. Journal of Power Sources 2017, 372, 16-23.

(4) Doyle, M.; Fuller, T. F.; Newman, J. Modeling of galvanostatic charge and discharge of the lithium/polymer/insertion cell. Journal of the Electrochemical society 1993, 140,1526

(5) Fuller, T. F.; Doyle, M.; Newman, J. Simulation and optimization of the dual lithium ion insertion cell. Journal of the Electrochemical Society 1994, 141, 1.

(6) Fuller, T. F.; Doyle, M.; Newman, J. S. Relaxation Phenomena in Lithium-IonInsertion Cells. Journal of The Electrochemical Society 1994, 141, 982. 
(7) Doyle, M.; Newman, J. The use of mathematical modeling in the design of lithium/polymer battery systems. Electrochimica Acta 1995, 40, 2191-2196.

(8) Newman, J.; Thomas-Alyea, K. E. Electrochemical Systems; Wiley-Interscience, 2004; Vol. 3; pp 1-647.

(9) Van der Ven, A.; Deng, Z.; Banerjee, S.; Ong, S. P. Rechargeable Alkali-Ion Battery Materials: Theory and Computation. Chemical Reviews 2020, 120, 6977-7019.

(10) Shi, S.; Gao, J.; Liu, Y.; Zhao, Y.; Wu, Q.; Ju, W.; Ouyang, C.; Xiao, R. Multiscale computation methods: Their applications in lithium-ion battery research and development. Chinese Physics B 2016, 25, 018212.

(11) Franco, A. A. Multiscale modelling and numerical simulation of rechargeable lithium ion batteries: concepts, methods and challenges. Rsc Advances 2013, 3, 13027-13058.

(12) Herbert, J. M. Dielectric continuum methods for quantum chemistry. Wiley Interdisciplinary Reviews: Computational Molecular Science 2021, 1-73.

(13) Scrosati, B. Lithium Rocking Chair Batteries: An Old Concept? Journal of The Electrochemical Society 1992, 139, 2776-2781.

(14) Asenbauer, J.; Eisenmann, T.; Kuenzel, M.; Kazzazi, A.; Chen, Z.; Bresser, D. The success story of graphite as a lithium-ion anode material - fundamentals, remaining challenges, and recent developments including silicon (oxide) composites. Sustainable Energy \& Fuels 2020, 10.1039.D0SE00175A.

(15) Zhang, H.; Yang, Y.; Ren, D.; Wang, L.; He, X. Graphite as anode materials: Fundamental mechanism, recent progress and advances. Energy Storage Materials 2021, $36,147-170$.

(16) Wang, A.; Kadam, S.; Li, H.; Shi, S.; Qi, Y. Review on modeling of the anode solid 

electrolyte interphase (SEI) for lithium-ion batteries. npj Computational Materials 2018, 4.

(17) Shepherd, K.; Siddiqui, F. A driverless Tesla crashed and burned for four hours, police said, killing two passengers in Texas. Washington Post

(18) Pfrang, A.; Kriston, A.; Ruiz, V.; Lebedeva, N.; di Persio, F. Emerging Nanotechnologies in Rechargeable Energy Storage Systems; Elsevier Inc., 2017; pp 253-290.

(19) Liu, L.; Xu, J.; Wang, S.; Wu, F.; Li, H.; Chen, L. Practical evaluation of energy densities for sulfide solid-state batteries. eTransportation 2019, 1, 100010.

(20) Galiński, M.; Lewandowski, A.; Stępniak, I. Ionic liquids as electrolytes. Electrochimica acta 2006, 51, 5567-5580.

(21) Wang, Y.; Meng, X.; Sun, J.; Liu, Y. Hou, L. Recent Progress in "Water-in-Salt" Electrolytes Toward Non-lithium Based Rechargeable Batteries. Frontiers in Chemistry 2020, 8 .

(22) Logan, E.; Dahn, J. Electrolyte Design for Fast-Charging Li-Ion Batteries. Trends in Chemistry 2020, 2, 354-366.

(23) Woods, L. Technological Advancements in Solid State Batteries for Electric Vehicles, 2020 Report - Toyota is the Pioneer of Solid-state Battery Research and Development with a Lion's Share of Patent Ownership. 2021.

(24) Zhu, Y; He, X.; Mo, Y. Origin of Outstanding Stability in the Lithium Solid Electrolyte Materials: Insights from Thermodynamic Analyses Based on First-Principles Calculations. ACS Applied Materials \&f Interfaces 2015, 7, 23685-23693.

(25) Zhang, Q.; Cao, D.; Ma, Y.; Natan, A.; Aurora, P.; Zhu, H. Sulfide-Based Solid-State Electrolytes: Synthesis, Stability, and Potential for All-Solid-State Batteries. Advanced Materials 2019, 31, 1901131. 
3141

3142

3143

3144

3145

3146

3147

3148

3149

3150

3151

3152

3153

3154

3155

3156

3157

3158

3159

3160

3161

3162

3163

(26) Zhang, Z.; Shao, Y.; Lotsch, B.; Hu, Y. S.; Li, H.; Janek, J.; Nazar, L. F.; Nan, C. W.;

Maier, J.; Armand, M.; Chen, L. New horizons for inorganic solid state ion conductors. Energy \& Environmental Science 2018, 11, 1945-1976.

(27) Gurung, A.; Pokharel, J.; Baniya, A.; Pathak, R.; Chen, K.; Lamsal, B. S.; Ghimire, N.; Zhang, W.-H.; Zhou, Y.; Qiao, Q. A review on strategies addressing interface incompatibilities in inorganic all-solid-state lithium batteries. Sustainable Energy \& Fuels 2019, 3, 3279-3309.

(28) Xiao, Y.; Wang, Y.; Bo, S. H.; Kim, J. C.; Miara, L. J.; Ceder, G. Understanding interface stability in solid-state batteries. Nature Reviews Materials 2020, 5, 105-126.

(29) Xu, L.; Tang, S.; Cheng, Y.; Wang, K.; Liang, J.; Liu, C.; Cao, Y.-C.; Wei, F.; Mai, L. Interfaces in Solid-State Lithium Batteries. Joule 2018, 2, 1991-2015.

(30) Tateyama, Y.; Gao, B.; Jalem, R.; Haruyama, J. Theoretical picture of positive electrode-solid electrolyte interface in all-solid-state battery from electrochemistry and semiconductor physics viewpoints. Current Opinion in Electrochemistry 2019, 17, $149-157$.

(31) Ceder, G.; Ong, S. P.; Wang, Y. Predictive modeling and design rules for solid electrolytes. MRS Bulletin 2018, 43, 782-788.

(32) Ma, Y. Computer simulation of cathode materials for lithium ion and lithium batteries: A review. Energy \&3 Environmental Materials 2018, 1, 148-173.

(33) Yan, L.-M.; Su, J.-M.; Sun, C.; Yue, B.-H. Review of the first principles calculations and the design of cathode materials for Li-ion batteries. Advances in Manufacturing 2014, 2, 358-368.

(34) Wang, L.; Chen, B.; Ma, J.; Cui, G.; Chen, L. Reviving lithium cobalt oxide-based 

lithium secondary batteries-toward a higher energy density. Chemical Society Reviews 2018, 47, 6505-6602.

(35) Maleki Kheimeh Sari, H.; Li, X. Controllable cathode-electrolyte interface of $\mathrm{Li}\left[\mathrm{Ni}_{0.8} \mathrm{Co}_{0.1} \mathrm{Mn}_{0.1}\right] \mathrm{O}_{2}$ for lithium ion batteries: a review. Advanced Energy Materials 2019, 9, 1901597.

(36) Parr, R. G.; Yang, W. Density-Functional Theory of Atoms and Molecules (International Series of Monographs on Chemistry); Oxford University Press, USA, 1994.

(37) Hohenberg, P.; Kohn, W. Inhomogeneous Electron Gas. Phys. Rev. 1964, 136, B864B871.

(38) Kohn, W.; Sham, L. J. Self-Consistent Equations Including Exchange and Correlation Effects. Phys. Rev. 1965, 140, A1133-A1138.

(39) Dirac, P. A. M. Note on Exchange Phenomena in the Thomas Atom. Mathematical Proceedings of the Cambridge Philosophical Society 1930, 26, 376-385.

(40) Perdew, J. P.; Wang, Y. Accurate and simple analytic representation of the electrongas correlation energy. Phys. Rev! B 1992, 45, 13244-13249.

(41) Perdew, J. P.; Burke, K.; Ernzerhof, M. Generalized Gradient Approximation Made Simple. Phys. Rev. Lett. 1996, 77, 3865-3868.

(42) Becke, A. D. Density-functional exchange-energy approximation with correct asymptotic behavior. Phys. Rev. A 1988, 38, 3098-3100.

(43) Lee, C.; Yang, W.; Parr, R. G. Development of the Colle-Salvetti correlation-energy formula into a functional of the electron density. Phys. Rev. B 1988, 37, 785-789.

(44) Perdew, J. P.; Kurth, S.; Zupan, A.; Blaha, P. Accurate density functional with correct formal properties: A step beyond the generalized gradient approximation. Physical 
(45) Grimme, S. Semiempirical GGA-type density functional constructed with a long-range dispersion correction. Journal of Computational Chemistry 2006, 27, 1787-1799.

(46) Grimme, S.; Antony, J.; Ehrlich, S.; Krieg, H. A consistent and accurate ab initio parametrization of density functional dispersion correction (DFT-D) for the 94 elements H-Pu. The Journal of Chemical Physics 2010, 132, 154104.

(47) Caldeweyher, E.; Bannwarth, C.; Grimme, S. Extension of the D3 dispersion coefficient model. The Journal of Chemical Physics 2017, 14 7, 034112.

(48) Grimme, S.; Ehrlich, S.; Goerigk, L. Effect of the damping function in dispersion corrected density functional theory. Journal of Computational Chemistry 2011, 32, $1456-1465$.

(49) Johnson, E. R.; Becke, A. D. A post-Hartree-Fock model of intermolecular interactions: Inclusion of higher-order corrections. The Journal of Chemical Physics 2006, 124, 174104.

(50) Becke, A. D. Density-functional thermochemistry. III. The role of exact exchange. The Journal of Chemical Physics 1993, 98, 5648-5652.

(51) Cramer, C. J. Essentials of computational chemistry: theories and models; John Wiley \& Sons, 2013.

(52) Bredow, T; Gerson, A. R. Effect of exchange and correlation on bulk properties of MgO, NiO, and CoO. Phys. Rev. B 2000, 61, 5194-5201.

(53) Islam, M. M.; Bredow, T.; Gerson, A. Electronic properties of oxygen-deficient and aluminum-doped rutile $\mathrm{TiO}_{2}$ from first principles. Phys. Rev. B 2007, 76, 045217.

(54) Heyd, J.; Scuseria, G. E.; Ernzerhof, M. Hybrid functionals based on a screened Coulomb potential. The Journal of Chemical Physics 2003, 118, 8207-8215. 
3211 (55) Dudarev, S. L.; Botton, G. A.; Savrasov, S. Y.; Humphreys, C. J.; Sutton, A. P.

3212

3213

3214

3215

3216

3217

3218

3219

3220

3221

3222

3223

3224

3225

3226

3227

3228

3229

3230

3231

3232

3233 Electron-energy-loss spectra and the structural stability of nickel oxide: An LSDA+U study. Phys. Rev. B 1998, 57, 1505-1509.

(56) Dudarev, S. L.; Liechtenstein, A. I.; Castell, M. R.; Briggs, G. A. D.; Sutton, A. P. Surface states on $\mathrm{NiO}(100)$ and the origin of the contrast reversal in atomically resolved scanning tunneling microscope images. Phys. Rev, B 1997, 56, 4900-4908.

(57) Anisimov, V. I.; Aryasetiawan, F.; Lichtenstein, A. I. First-principles calculations of the electronic structure and spectra of strongly correlated systems: the $\mathbf{L D A}+U$ method. Journal of Physics: Condensed Matter 1997, 9, 767-808.

(58) Goedecker, S. Linear scaling electronic structure methods. Reviews of Modern Physics 1999, 71, 1085-1123.

(59) Prodan, E.; Kohn, W. Nearsightedness of electronic matter. Proceedings of the $\mathrm{Na}^{-}$ tional Academy of Sciences 2005, 102, 11635-11638.

(60) Galli, G.; Parrinello, M. Large scale electronic structure calculations. Phys. Rev. Lett. 1992, 69, 3547-3550.

(61) Hernández, E.; Gillan, M. J. Self-consistent first-principles technique with linear scaling. Phys. Rev. B 1995, 51, 10157-10160.

(62) Skylaris, C. K.; Haynes, P. D.; Mostofi, A. A.; Payne, M. C. Introducing ONETEP: Linear-scaling density functional simulations on parallel computers. Journal of Chemical Physics 2005, 122, 1-10.

(63) Gillan, M. J.; Bowler, D. R.; Torralba, A. S.; Miyazaki, T. Order-N first-principles calculations with the conquest code. Computer Physics Communications 2007, 17r, 
3234

3235

3236

3237

3238

3239

3240

3241

3242

3243

3244

3245

3246

3247

3248

3249

3250

3251

3252

3253

3254

3255

3256

(64) Mohr, S.; Ratcliff, L. E.; Genovese, L.; Caliste, D.; Boulanger, P.; Goedecker, S.; Deutsch, T. Accurate and efficient linear scaling DFT calculations with universal applicability. Physical Chemistry Chemical Physics 2015, 17, 31360-31370.

(65) A., P. J. C. The ONETEP linear-scaling density functional theory program, J. Chem. Phys. 2020, 152, 174111.

(66) Bhandari, A.; Anton, L.; Dziedzic, J.; Peng, C.; Kramer, D.; Skylaris, C.-K. Electronic structure calculations in electrolyte solutions: Methods for neutralization of extended charged interfaces. The Journal of Chemical Physics 2020, 153, 124101.

(67) Jónsson, H.; Mills, G.; Jacobsen, K. W. Nudged elastic band method for finding minimum energy paths of transitions. Classical and Quantum Dynamics in Condensed Phase Simulations 1998, 385-404.

(68) Henkelman, G.; Uberuaga, B. P.; Jónsson, H. A climbing image nudged elastic band method for finding saddle points and minimum energy paths. The Journal of chemical physics 2000, 113, 9901-9904.

(69) Henkelman, G.; Jónsson, H. Improved tangent estimate in the nudged elastic band method for finding minimum energy paths and saddle points. The Journal of chemical physics 2000, 113, 9978-9985.

(70) Peng, C.; Mercer, M. P.; Skylaris, C.-K.; Kramer, D. Lithium intercalation edge effects and doping implications for graphite anodes. Journal of Materials Chemistry A $\mathbf{2 0 2 0}$, $8,7947-7955$.

(71) Mercer, M. P.; Peng, C.; Soares, C.; Hoster, H. E.; Kramer, D. Voltage hysteresis during lithiation/delithiation of graphite associated with meta-stable carbon stackings. J. Mater. Chem. A 2021, 9, 492-504. 
(72) Sanchez, J. M.; Ducastelle, F.; Gratias, D. Generalized cluster description of multicomponent systems. Physica A: Statistical Mechanics and its Applications 1984, 128, $334-350$.

(73) De Fontaine, D. Cluster approach to order-disorder transformations in alloys. Solid state physics 1994, 47, 33-176.

(74) Blum, V.; Zunger, A. Mixed-basis cluster expansion for thermodynamics of bcc alloys. Physical Review B 2004, 70, 155108.

(75) Gallavotti, G. Statistical mechanics: A short treatise; Springer Science \& Business Media, 2013.

(76) Persson, K.; Hinuma, Y.; Meng, Y. S.; Van der Ven, A.; Ceder, G. Thermodynamic and kinetic properties of the Li-graphite system from first-principles calculations. Phys. Rev. B 2010, 82, 125416.

(77) Van der Ven, A.; Ceder, G.; Asta, M.; Tepesch, P. D. First-principles theory of ionic diffusion with nondilute carriers. Phys. Rev. B 2001, 64, 184307.

(78) van de Walle, A. Multicomponent multisublattice alloys, nonconfigurational entropy and other additions to the Alloy Theoretic Automated Toolkit. Calphad 2009, 33, $266-278$.

(79) van de Walle, A.; Asta, M. D.; Ceder, G. The Alloy Theoretic Automated Toolkit: A User Guide. Calphad 2002, 26, 539-553.

(80) van de Walle, A.; Ceder, G. Automating first-principles phase diagram calculations. Journal of Phase Equilibria 2002, 23, 348-359.

(81) Natarajan, A. R.; Thomas, J. C.; Puchala, B.; Van der Ven, A. Symmetry-adapted order parameters and free energies for solids undergoing order-disorder phase transitions. 
3281

3282

3283

3284

3285

3286

3287

3288

3289

3290

3291

3292

3293

3294

3295

3296

3297

3298

3299

3300

3301

3302

3303

(82) Pickard, C. J.; Needs, R. J. Ab initiorandom structure searching. Journal of Physics: Condensed Matter 2011, 23, 053201.

(83) Ångqvist, M.; Muñoz, W. A.; Rahm, J. M.; Fransson, E.; Durniak, C.; Rozyezko, P.; Rod, T. H.; Erhart, P. ICET - A Python Library for Constructing and Sampling Alloy Cluster Expansions. Advanced Theory and Simulations 2019, 2, 1900015.

(84) Chang, J. H.; Kleiven, D.; Melander, M.; Akola, J.; Garcia-Lastra, J. M.; Vegge, T. CLEASE: a versatile and user-friendly implementation of cluster expansion method. Journal of Physics: Condensed Matter 2019, 31, 325901.

(85) Binder, K.; Landau, D. P. A Guide to Monte-Carlo Simulations in Statistical Physics, Third Edition; Cambridge University Press: Cambridge, UK, 2009.

(86) Lee, T. D.; Yang, C. N. Statistical Theory of Equations of State and Phase Transitions. II. Lattice Gas and Ising Model. Phys. Rev. 1952, 87, 410-419.

(87) Mercer, M. P.; Hoster, H. E. Ultrahigh vacuum and electrocatalysis - The powers of quantitative surface imaging. Nano Energy 2016, 29, 394-413.

(88) Oviedo, O.; Reinaudi, L.; Garcia, S.; Leiva, E. Underpotential Deposition: From Fundamentals and Theory to Applications at the Nanoscale; Monographs in Electrochemistry; Springer International Publishing, 2015.

(89) Mercer, M. P.; Finnigan, S.; Kramer, D.; Richards, D.; Hoster, H. E. The influence of point defects on the entropy profiles of Lithium Ion Battery cathodes: a lattice-gas Monte Carlo study. Electrochimica Acta 2017, 241, 141-152.

(90) Kim, S.-W.; Pyun, S.-I. Thermodynamic and kinetic approaches to lithium intercalation into a $\mathrm{Li}_{1-\delta} \mathrm{Mn}_{2} \mathrm{O}_{4}$ electrode using Monte Carlo simulation. Electrochimica Acta 2001, 46, 987-997. 
(99) Darling, R.; Newman, J. Dynamic Monte Carlo Simulations of Diffusion in $\mathrm{Li}_{y} \mathrm{Mn}_{2} \mathrm{O}_{4}$. Journal of The Electrochemical Society 1999, 146, 3765-3772.

(100) Thomas, K. E.; Newman, J. Heats of mixing and of entropy in porous insertion electrodes. Journal of Power Sources 2003, 119-121, 844-849.

(101) Zhang, X.-F.; Zhao, Y.; Patel, Y.; Zhang, T.; Liu, W.-M.; Chen, M.; Offer, G. J.; Yan, Y. Potentiometric measurement of entropy change for lithium batteries. Phys. Chem. Chem. Phys. 2017, 19, 9833-9842.

(102) Gavilán-Arriazu, E.; Pinto, O.; López de Mishima, B.; Barraco, D.; Oviedo, O.; Leiva, E. Kinetic Monte Carlo applied to the electrochemical study of the Li-ion graphite system. Electrochimica Acta 2020, 331, 135439.

(103) Gavilán-Arriazu, E. M.; Mercer, M.P.; Pinto, O. A.; Oviedo, O. A.; Barraco, D. E.; Hoster, H. E.; Leiva, E. P. M. Effect of Temperature on The Kinetics of Electrochemical Insertion of Li-Ions into a Graphite Eleetrode Studied by Kinetic Monte Carlo. Journal of The Electrochemical Society 2020, 167, 013533.

(104) Gavilán-Arriazu, E. M.; Mercer, M. P.; Barraco, D. E.; Hoster, H. E.; Leiva, E. P. M. Kinetic Monte Carlo simulations applied to Li-ion and post Li-ion batteries: a key link in the multi-scale chain. Progress in Energy 2021, 3, 042001.

(105) Car, R.; Parrinello, M. Unified Approach for Molecular Dynamics and DensityFunctional Theory. Physical Review Letters 1985, 55, 2471-2474.

(106) Tuckerman, M. E.; Parrinello, M. Integrating the Car-Parrinello equations. I. Basic integration techniques. The Journal of Chemical Physics 1994, 101, 1302-1315.

(107) Grossman, J. C.; Schwegler, E.; Draeger, E. W.; Gygi, F.; Galli, G. Towards an assessment of the accuracy of density functional theory for first principles simulations of water. The Journal of Chemical Physics 2004, 120, 300-311. 
3352

3353

3354

3355

3356

3357

3358

3359

3360

3361

3362

3363

3364

3365

3366

3367

3368

3369

3370

3371

3372

3373

3374

(108) Harding, J. H. Computer simulation of defects in ionic solids. Reports on Progress in Physics 1990, 53, 1403-1466.

(109) Buckingham, R. A. The classical equation of state of gaseous helium, neon and argon. Proceedings of the Royal Society of London. Series A. Mathematical and Physical Sciences 1938, 168, 264-283.

(110) Born, M.; Mayer, J. On the Lattice Theory of Ionic Crystals. Zeitschrift für Physik 1932, $75,1-18$.

(111) Mayer, J. E. Dispersion and Polarizability and the Van der Waals Potential in the Alkali Halides. The Journal of Chemical Physics 1932, 1, 270.

(112) Todorov, I. T.; Smith, W.; Trachenko, K.; Dove, M. T. DL_POLY_3: new dimensions in molecular dynamics simulations via massive parallelism. Journal of Materials Chemistry 2006, 16, 1911-1918.

(113) Plimpton, S. Fast Parallel Algorithms for Short-Range Molecular Dynamics. Journal of Computational Physics 1995, 117, 1 - 19.

(114) Gale, J. D. GULP: A computer program for the symmetry-adapted simulation of solids. Journal of the Chemical Society, Faraday Transactions 1997, 93, 629-637.

(115) Gauthier, M.; Carney, T. J.; Grimaud, A.; Giordano, L.; Pour, N.; Chang, H.-H.; Fenning, D. P.; Lux, S. F.; Paschos, O.; Bauer, C.; Maglia, F.; Lupart, S.; Lamp, P.; Shao-Horn, Y. The Electrode-Electrolyte Interface in Li-ion Batteries: Current Understanding and New Insights. The Journal of Physical Chemistry Letters 2015, 4653-4672 .

(116) Yu, X.; Manthiram, A. Electrode-electrolyte interfaces in lithium-based batteries. Energy \& Environmental Science 2018, 11, 527-543. 
(117) Jinnouchi, R.; Kodama, K.; Morimoto, Y. Electronic structure calculations on electrolyte-electrode interfaces: Successes and limitations. Current Opinion in Electrochemistry 2018, 8, 103-109.

(118) Hansen, M. H.; Rossmeisl, J. PH in Grand Canonical Statistics of an Electrochemical Interface. Journal of Physical Chemistry C 2016, 120, 29135-29143.

(119) Sakong, S.; Naderian, M.; Mathew, K.; Hennig, R. G.; Groß, A. Density functional theory study of the electrochemical interface between a Pt electrode and an aqueous electrolyte using an implicit solvent method. Journal of Chemical Physics 2015, 142.

(120) Skyner, R. E.; McDonagh, J. L.; Groom, C. R.; Van Mourik, T.; Mitchell, J. B. A review of methods for the calculation of solution free energies and the modelling of systems in solution. Physical Chemistry Chemical Physics 2015, 17, 6174-6191.

(121) Kang, J.; Wei, S. H.; Zhu, K.; Kim, Y. H. First-principles theory of electrochemical capacitance of nanostructured materials: Dipole-assisted subsurface intercalation of lithium in pseudocapacitive $\mathrm{TiO}_{2}$ anatase nanosheets. Journal of Physical Chemistry C 2011, 115, 4909-4915.

(122) Dufils, T.; Jeanmairet, G.; Rotenberg, B.; Sprik, M.; Salanne, M. Simulating Electrochemical Systems by Combining the Finite Field Method with a Constant Potential Electrode. Physical Review Letters 2019, 123, 195501.

(123) Jorn, R.; Kumar, R; Abraham, D. P.; Voth, G. A. Atomistic modeling of the electrodeelectrolyte interface in Li-ion energy storage systems: Electrolyte structuring. Journal of Physical Chemistry C 2013, 117, 3747-3761.

(124) Zhang, C.; Sayer, T.; Hutter, J.; Sprik, M. Modelling electrochemical systems with finite field molecular dynamics. Journal of Physics: Energy 2020, 0-21. 
(125) Cramer, C. J.; Truhlar, D. G. Implicit Solvation Models: Equilibria, Structure, Spectra, and Dynamics. Chemical Reviews 1999, 99, 2161-2200.

(126) Tomasi, J.; Mennucci, B.; Cammi, R. Quantum mechanical continuum solvation models. Chemical Reviews 2005, 105, 2999-3093.

(127) Grochowski, P.; Trylska, J. Continuum molecular electrostatics, salt effects, and counterion binding - A review of the Poisson-Boltzmann theory and its modifications. Biopolymers 2008, 89, 93-113.

(128) Jinnouchi, R.; Anderson, A. B. Electronic structure calculations of liquid-solid interfaces: Combination of density functional theory and modified Poisson-Boltzmann theory. Phys. Rev. B 2008, 7r, 245417.

(129) Gunceler, D.; Letchworth-Weaver, K.; Sundararaman, R.; Schwarz, K. A.; Arias, T. A. The importance of nonlinear fluid response in joint density-functional theory studies of battery systems. Modelling and Simulation in Materials Science and Engineering 2013, 21.

(130) Ringe, S.; Oberhofer, H.; Hille, C.; Matera, S.; Reuter, K. Function-Space-Based Solution Scheme for the Size-Modified Poisson-Boltzmann Equation in Full-Potential DFT. Journal of Chemical Theory and Computation 2016, 12, 4052-4066.

(131) Nattino, F.; Truscott, M.; Marzari, N.; Andreussi, O. Continuum models of the electrochemical diffuse layer in electronic-structure calculations. The Journal of Chemical Physics 2019, 150, 41722.

(132) Melander, M. M.; Kuisma, M. J.; Christensen, T. E. K.; Honkala, K. Grand-canonical approach to density functional theory of electrocatalytic systems: Thermodynamics of solid-liquid interfaces at constant ion and electrode potentials. Journal of Chemical Physics 2019, 150, 041706. 
3422

3423

3424

3425

3426

3427

3428

3429

3430

3431

3432

3433

3434

3435

3436

3437

3438

3439

3440

3441

3442

3443

3444

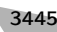

(133) Stein, C. J.; Herbert, J. M.; Head-Gordon, M. The Poisson-Boltzmann model for implicit solvation of electrolyte solutions: Quantum chemical implementation and assessment via Sechenov coefficients. Journal of Chemical Physics 2019, 151, 22411.

(134) Womack, J. C.; Anton, L.; Dziedzic, J.; Hasnip, P. J.; Probert, M. I.; Skylaris, C. K. DL-MG: A Parallel Multigrid Poisson and Poisson-Boltzmann Solver for Electronic Structure Calculations in Vacuum and Solution. Journal of Chemical Theory and Computation 2018, 14, 1412-1432.

(135) Dziedzic, J.; Bhandari, A.; Anton, L.; Peng, C.; Womack, J. C.; Famili, M.; Kramer, D.; Skylaris, C.-K. A Practical Approach to Large Scale Electronic Structure Calculations in Electrolyte Solutions via Continuum-Embedded Linear-Scaling DFT. The Journal of Physical Chemistry C 2020, 124, 7860-7872.

(136) Andreussi, O.; Dabo, I.; Marzari, N. Revised self-consistent continuum solvation in electronic-structure calculations. Journal of Chemical Physics 2012, 136, 064102.

(137) Fisicaro, G.; Genovese, L.; Andreussi, O.; Mandal, S.; Nair, N. N.; Marzari, N.; Goedecker, S. Soft-Sphere Continuum Solvation in Electronic-Structure Calculations. Journal of Chemical Theory and Computation 2017, 13, 3829-3845.

(138) Sundararaman, R.; Letchworth-Weaver, K.; Schwarz, K. A. Improving accuracy of electrochemical capacitance and solvation energetics in first-principles calculations. Journal of Chemical Physics 2018, 148, 144105.

(139) Dziedzic, J.; Helal, H. H.; Skylaris, C. K.; Mostofi, A. A.; Payne, M. C. Minimal parameter implicit solvent model for ab initio electronic-structure calculations. Epl 2011, 95, 1-6.

(140) Jones, J. E. On the determination of molecular fields. - II. From the equation of state of a gas. Proceedings of the Royal Society of London. Series A, Containing Papers of ${ }_{3446}$ a Mathematical and Physical Character 1924, 106, 463-477. 
3447

3448

3449

3450

3451

3452

3453

3454

3455

3456

3457

3458

3459

3460

3461

3462

3463

3464

3465

3466

3467

3468

(141) Gale, J. D. GULP fitting. 1996; https://gulp.curtin.edu.au/local/docs/gulp/ gulp_31_manual/gulpnode38.html.

(142) Gale, J. D. Empirical potential derivation for ionic materials. Philosophical Magazine B 1996, 73, 3-19.

(143) Stukowski, A.; Fransson, E.; Mock, M.; Erhart, P. Atomicrex-a general purpose tool for the construction of atomic interaction models. Modelling and Simulation in Materials Science and Engineering 2017, 25, 055003.

(144) Ostrouchov, C. dftfit. https://chrisostrouchov.com/dftfit.

(145) Wen, M.; Li, J.; Brommer, P.; Elliott, R. S.; Sethna, J. P.; Tadmor, E. B. A KIMcompliant potfit for fitting sloppy interatomic potentials: application to the EDIP model for silicon. Modelling and Simulation in Materials Science and Engineering 2017, 25, 014001.

(146) Wen, M.; Li, J.; Brommer, P.; Elliott, R. S.; Sethna, J. P.; Tadmor, E. B. potfit. https://www.potfit.net/wiki/doku.php.

(147) Lewis, G. V.; Catlow, C. R. A. Potential models for ionic oxides. Journal of Physics C: Solid State Physics 1985, 18, 1149-1161.

(148) Ledwaba, R. S.; Sayle, D. C.; Ngoepe, P. E. Atomistic Simulation and Characterization of Spinel $\mathrm{Li}_{1+x} \mathrm{Mn}_{2} \mathrm{O}_{4}(0 \leq x \leq 1)$ Nanoparticles. ACS Applied Energy Materials 2020, 3, 1429-1438.

(149) Sayle, T. X. T.; Catlow, C. R. A.; Maphanga, R. R.; Ngoepe, P. E.; Sayle, D. C. Generating $\mathrm{MnO}_{2}$ Nanoparticles Using Simulated Amorphization and Recrystallization. Journal of the American Chemical Society 2005, 127, 12828-12837.

(150) Dawson, J. A.; Tanaka, I. Oxygen Vacancy Formation and Reduction Properties of 
3470

3471

3472

3473

3474

3475

3476

3477

3478

3479

3480

3481

3482

3483

3484

3485

3486

3487

3488

3489

3490

3491

3492

$\beta-\mathrm{MnO}_{2}$ Grain Boundaries and the Potential for High Electrochemical Performance. ACS Applied Materials \& Interfaces 2014, 6, 17776-17784.

(151) Hart, F. X.; Bates, J. B. Lattice model calculation of the strain energy density and other properties of crystalline $\mathrm{LiCoO}_{2}$. Journal of Applied Physics 1998, 83, 75607566.

(152) A. J. Fisher, C.; Saiful Islam, M.; Moriwake, H. Atomic Level Investigations of Lithium Ion Battery Cathode Materials. Journal of the Physical Society of Japan 2010, 79, $59-64$.

(153) Ammundsen, B.; Burns, G. R.; Islam, M. S.; Kanoh, H.; Rozière, J. Lattice Dynamics and Vibrational Spectra of Lithium Manganese Oxidés: A Computer Simulation and Spectroscopic Study. The Journal of Physical Chemistry B 1999, 103, 5175-5180.

(154) Kerisit, S.; Chaka, A. M.; Droubay, T. C; flton, E. S. Shell Model for Atomistic Simulation of Lithium Diffusion in Mixed Mn/Ti Oxides. The Journal of Physical Chemistry C 2014, 118, 24231-24239.

(155) He, J.; Zhang, L.; Liu, L. Thermal transport in monocrystalline and polycrystalline lithium cobalt oxide. Physical Chemistry Chemical Physics 2019, 21, 12192-12200.

(156) Lee, S.; Park, S. S. Atomistic simulation study of mixed-metal oxide $\left(\mathrm{LiNi}_{1 / 3} \mathrm{Co}_{1 / 3} \mathrm{Mn}_{1 / 3} \mathrm{O}_{2}\right)$ cathode material for lithium ion battery. The Journal of Physical Chemistry C 2012, 116, 6484-6489.

(157) Lindan, P. J. D.; Gillan, M. J. Shell-model molecular dynamics simulation of superionic conduction in CaF2. Journal of Physics: Condensed Matter 1993, 5, 1019-1030.

(158) Mitchell, P. J.; Fincham, D. Shell model simulations by adiabatic dynamics. Journal of Physics: Condensed Matter 1993, 5, 1031-1038. 
(159) Wang, B.; Cormack, A. N. Molecular dynamics simulations of Mg-doped beta"-alumina with potential models fitted for accurate structural response to thermal vibrations. Solid State Ionics 2014, 263, 9-14.

(160) Escribano, B.; Lozano, A.; Radivojević, T.; Fernández-Pendás, M.; Carrasco, J.; Akhmatskaya, E. Enhancing sampling in atomistic simulations of solid-state materials for batteries: a focus on olivine $\mathrm{NaFePO}_{4}$. Theoretical Chemistry Accounts 2017, $136,43$.

(161) Lee, S.; Park, S. S. Lithium transition metal fluorophosphates $\left(\mathrm{Li}_{2} \mathrm{CoPO}_{4} \mathrm{~F}\right.$ and $\left.\mathrm{Li}_{2} \mathrm{NiPO}_{4} \mathrm{~F}\right)$ as cathode materials for lithium ion battery from atomistic simulation. Journal of Solid State Chemistry 2013, 204, 329-334.

(162) Dai, J.; Chen, Q.; Glossmann, T.; Lai, W. Comparison of interatomic potential models on the molecular dynamics simulation of fast-ion conductors: A case study of a $\mathrm{Li}$ garnet oxide $\mathrm{Li}_{7} \mathrm{La}_{3} \mathrm{Zr}_{2} \mathrm{O}_{12}$. Computational Materials Science 2019, 162, 333-339.

(163) Pedone, A.; Malavasi, G.; Menziani, M. C.; Cormack, A. N.; Segre, U. A New SelfConsistent Empirical Interatomic Potential Model for Oxides, Silicates, and SilicaBased Glasses. The Journal of Physical Chemistry B 2006, 110, 11780-11795.

(164) Morgan, Lucy M. and Clarke, Matthew and Islam, M. Saiful and Morgan, Benjamin J., PopOff: POtential Parameter Optimisation for Force-Fields. 2021; https://doi. org/10.5281/zenodo.4773795.

(165) Fisher, C. A. J.; Hart Prieto, V. M.; Islam, M. S. Lithium Battery Materials $\mathrm{LiMPO}_{4}$ $(\mathrm{M}=\mathrm{Mn}, \mathrm{Fe}, \mathrm{Co}$,and $\mathrm{Ni})$ : Insights into Defect Association, Transport Mechanisms, and Doping Behavior. Chemistry of Materials 2008, 20, 5907-5915.

(166) Urban, A.; Seo, D.-H.; Ceder, G. Computational understanding of Li-ion batteries. npj Computational Materials 2016, 2, 16002. 
3517 (167) Ceder, G.; Van der Ven, A. Phase diagrams of lithium transition metal oxides: inves-

3518

3519

3520

3521

3522

3523

3524

3525

3526

3527

3528

3529

3530

3531

3532

3533

3534

3535

3536

3537 tigations from first principles. Electrochimica Acta 1999, 45, 131 - 150.

(168) Van Der Ven, A.; Marianetti, C.; Morgan, D.; Ceder, G. Phase transformations and volume changes in spinel $\mathrm{Li}_{x} \mathrm{Mn}_{2} \mathrm{O}_{4}$. Solid State Ionics 2000, 135, 21-32.

(169) Reynier, Y.; Graetz, J.; Swan-Wood, T.; Rez, P.; Yazami, R.; Fultz, B.' Entropy of Li intercalation in $\mathrm{Li}_{x} \mathrm{CoO}_{2}$. Phys. Rev. B 2004, 70, 174304.

(170) Yazami, R.; Reynier, Y. Thermodynamics and crystal structure anomalies in lithiumintercalated graphite. Journal of Power Sources 2006, 153, 312-318.

(171) Atkins, P. W.; De Paula, J. Atkins' Physical chemistry, 9th Ed.; 2014.

(172) Heitjans, P.; Kärger, J. Diffusion in condensed matter: methods, materials, models; Springer Science \& Business Media, 2006.

(173) Heumann, T. Diffusion in Metallen: Grundlagen, Theorie, Vorgänge in Reinmetallen und Legierungen; Springer-Verlag, 2013; Vol. 10.

(174) Crank, J. The mathematics of diffusion; Oxford university press, 1979.

(175) Einstein, A. The presumed movement of suspended particles in static fluids. Ann. Phys. Lpz 1905, 17, 549-560.

(176) Von Smoluchowski, M. Zur kinetischen theorie der brownschen molekularbewegung und der suspensionen. Annalen der physik 1906, 326, 756-780.

(177) Ladd, A. J.; Moran, B.; Hoover, W. G. Lattice thermal conductivity: A comparison of molecular dynamics and anharmonic lattice dynamics. Physical Review B 1986, 34 , 5058. 
3559

(178) Turney, J.; Landry, E.; McGaughey, A.; Amon, C. Predicting phonon properties and thermal conductivity from anharmonic lattice dynamics calculations and molecular dynamics simulations. Physical Review B 2009, 79, 064301.

(179) Seko, A.; Togo, A.; Hayashi, H.; Tsuda, K.; Chaput, L.; Tanaka, I. Prediction of lowthermal-conductivity compounds with first-principles anharmonic lattice-dynamics calculations and Bayesian optimization. Physical review letters 2015, 115, 205901.

(180) Togo, A.; Chaput, L.; Tanaka, I. Distributions of Phonon Lifetimes in Brillouin Zones. Phys. Rev. B 2015, 91, 094306.

(181) Yang, H.; Yang, J.-y.; Savory, C. N.; Skelton, J. M.; Morgan, B. J.; Scanlon, D. O.; Walsh, A. Highly Anisotropic Thermal Transport in $\mathrm{LiCoO}_{2}$. The journal of physical chemistry letters 2019, 10, 5552-5556.

(182) Yang, H.; Savory, C. N.; Morgan, B. J.; Scanlon, D. O.; Skelton, J. M.; Walsh, A. Chemical Trends in the Lattice Thermal Conductivity of Li (Ni, Mn, Co) O2 (NMC) Battery Cathodes. Chemistry of Materials 2020, 32, 7542-7550.

(183) Tadano, T.; Gohda, Y.; Tsuneyuki, S. Anharmonic force constants extracted from first-principles molecular dynamics: applications to heat transfer simulations. Journal of Physics: Condensed Matter 2014, 26, 225402.

(184) Li, W.; Carrete, J.; Katcho, N. A.; Mingo, N. ShengBTE: a solver of the Boltzmann transport equation for phonons. Comp. Phys. Commun. 2014, 185, 1747-1758.

(185) Vetter, J.; Novák, P.; Wagner, M.; Veit, C.; Möller, K.-C.; Besenhard, J.; Winter, M.; Wohlfahrt-Mehrens, M.; Vogler, C.; Hammouche, A. Ageing mechanisms in lithiumion batteries. Journal of Power Sources 2005, 147, $269-281$.

(186) Agubra, V.; Fergus, J. Lithium Ion Battery Anode Aging Mechanisms. Materials 2013, 6, 1310-1325. 
(187) Yang, X.-G.; Leng, Y.; Zhang, G.; Ge, S.; Wang, C.-Y. Modeling of lithium plating induced aging of lithium-ion batteries: Transition from linear to nonlinear aging. Journal of Power Sources 2017, 360, 28 - 40.

(188) Guerard, D.; Herold, A. Intercalation of lithium into graphite and other carbons. Carbon 1975, 13, $337-345$.

(189) Woo, K. C.; Kamitakahara, W. A.; DiVincenzo, D. P.; Robinson, D. S.; Mertwoy, H.; Milliken, J. W.; Fischer, J. E. Effect of In-Plane Density on the Structural and Elastic Properties of Graphite Intercalation Compounds. Phys. Rev.Lett. 1983, 50, 182-185.

(190) Basu, S.; Zeller, C.; Flanders, P.; Fuerst, C.; Johnson, W.; Fischer, J. Synthesis and properties of lithium-graphite intercalation compounds. Materials Science and Engineering 1979, 38, $275-283$.

(191) Besenhard, J. The electrochemical preparation and properties of ionic alkali metaland NR4-graphite intercalation compounds in organic electrolytes. Carbon 1976, 14, $111-115$.

(192) Yazami, R.; Touzain, P. A reversible graphite-lithium negative electrode for electrochemical generators. Journal of Power Sources 1983, 9, 365 - 371.

(193) Fong, R.; von Sácken, U.; Dahn, J. R. Studies of Lithium Intercalation into Carbons Using Nonaqueous Electrochemical Cells. Journal of The Electrochemical Society $1990,137,2009-2013$.

(194) Tarascon, J.; Guyomard, D. The $\mathrm{Li}_{1+x} \mathrm{Mn}_{2} \mathrm{O}_{4} / \mathrm{C}$ rocking-chair system: a review. Electrochimica Acta 1993, 38, 1221 - 1231.

(195) Dahn, J. R. Phase diagram of $\mathrm{Li}_{x} \mathrm{C}_{6}$. Physical Review B 1991, 44, 9170-9177.

(196) Hazrati, E.; de Wijs, G. A.; Brocks, G. Li intercalation in graphite: A van der Waals 3585 density-functional study. Physical Review B 2014, 90, 155448. 
(197) Konar, S.; Häusserman, U.; Svensson, G. Intercalation compounds from LiH and graphite: Relative stability of metastable stages and thermodynamic stability of dilute stage Id. Chemistry of Materials 2015, 27, 2566-2575.

(198) Shi, H.; Barker, J.; Saïdi, M. Y.; Koksbang, R. Structure and Lithium Intercalation Properties of Synthetic and Natural Graphite. Journal of The Electrochemical Society 1996, 143, 3466-3472.

(199) Sethuraman, V. A.; Hardwick, L. J.; Srinivasan, V.; Kostecki, R. Surface structural disordering in graphite upon lithium intercalation/deintercalation. Journal of Power Sources 2010, 195, 3655-3660.

(200) Ohzuku, T. Formation of Lithium-Graphite Intercalation Compounds in Nonaqueous Electrolytes and Their Application as a Negative Electrode for a Lithium Ion (Shuttlecock) Cell. Journal of The Electrochemical Society 1993, 140, 2490.

(201) He, H.; Huang, C.; Luo, C.-W.; Liu, J.-J.; Chao, Z.-S. Dynamic study of Li intercalation into graphite by in situ high energy synchrotron XRD. Electrochimica Acta 2013, 92, $148-152$.

(202) Trucano, P.; Chen, R. Structure of graphite by neutron diffraction. Nature 1975, 258, $136-137$.

(203) Okamoto, H. The C-Li (Carbon-Lithium) system. Bulletin of Alloy Phase Diagrams 1989, 10, $69-72$.

(204) Billaud, D.; Henry, F.; Lelaurain, M.; Willmann, P. Revisited structures of dense and dilute stage II lithium-graphite intercalation compounds. Journal of Physics and Chemistry of Solids 1996, 57, 775-781.

(205) Billaud, D.; Henry, F. Structural studies of the stage III lithium-graphite intercalation compound. Solid State Communications 2002, 124, 299 - 304. 
(206) Didier, C.; Pang, W. K.; Guo, Z.; Schmid, S.; Peterson, V. K. Phase Evolution and Intermittent Disorder in Electrochemically Lithiated Graphite Determined Using in Operando Neutron Diffraction. Chemistry of Materials 2020, 32, 2518-2531.

(207) Zheng, T.; Reimers, J. N.; Dahn, J. R. Effect of turbostratic disorder in graphitic carbon hosts on the intercalation of lithium. Physical Review B 1995, 51, 734-741.

(208) Senyshyn, A.; Dolotko, O.; Muhlbauer, M. J.; Nikolowski, K.; Fuess, H.; Ehrenberg, H. Lithium Intercalation into Graphitic Carbons Revisited: Experimental Evidence for Twisted Bilayer Behavior. Journal of the Electrochemical Society 2013, 160, A3198A3205.

(209) Taminato, S. et al. Real-time observations of lithium battery reactions - Operando neutron diffraction analysis during practical operation. Scientific Reports 2016, 6, 28843.

(210) Thinius, S.; Islam, M. M.; Heitjans, P.; Bredow, T. Theoretical study of Li migration in lithium-graphite intercalation compounds with dispersion-corrected DFT methods. The Journal of Physical Chemistry C 2014, 118, 2273-2280.

(211) Garcia, J. C.; Bloom, I.; Johnson, C.; Dees, D.; Iddir, H. Graphite Lithiation under Fast Charging Conditions: Atomistic Modeling Insights. The Journal of Physical Chemistry C 2020, 124, 8162-8169.

(212) Olsson, E.; Cottom, J.; Au, H.; Guo, Z.; Jensen, A. C. S.; Alptekin, H.; Drew, A. J.; Titirici, M.-M.; Cai, Q. Elucidating the Effect of Planar Graphitic Layers and Cylindrical Pores on the Storage and Diffusion of Li, Na, and $\mathrm{K}$ in Carbon Materials. Advanced Functional Materials 2020, 30, 1908209.

(213) Lenchuk, O.; Adelhelm, P.; Mollenhauer, D. Comparative study of density functionals for the description of lithium-graphite intercalation compounds. Journal of Computational Chemistry 2019, 40, 2400-2412. 
(214) Ji, X.; Wang, Y.; Zhang, J. Understanding the anisotropic strain effects on lithium diffusion in graphite anodes: A first-principles study. Physica B: Condensed Matter 2018, 539, $66-71$.

(215) Wang, Z.; Ratvik, A. P.; Grande, T.; Selbach, S. M. Diffusion of alkali metals in the first stage graphite intercalation compounds by vdW-DFT calculations. $R S C A d v$. 2015, 5, 15985-15992.

(216) Persson, K.; Sethuraman, V. A.; Hardwick, L. J.; Hinuma, Y.; Meng, Y. S.; Van Der Ven, A.; Srinivasan, V.; Kostecki, R.; Ceder, G. Lithium diffusion in graphitic carbon. The journal of physical chemistry letters 2010, 1, 1176-1180.

(217) Otero, M.; Sigal, A.; Perassi, E.; Barraco, D.; Leiva, E.Statistical mechanical modeling of the transition Stage II $\rightarrow$ Stage I of Li-ion storage in graphite. A priori vs induced heterogeneity. Electrochimica Acta 2017, 245, 569 - 574.

(218) Gavilán-Arriazu, E. M.; Pinto, O. A.; de Mishima, B. A. L.; Leiva, E. P. M.; Oviedo, O. A. Grand Canonical Monte Carlo Study of Li Intercalation into Graphite. Journal of The Electrochemical Society 2018, 165, A2019-A2025.

(219) Gavilán Arriazu, E. M.; López de Mishima, B. A.; Oviedo, O. A.; Leiva, E. P. M.; Pinto, O. A. Criticality of the phase transition on stage two in a lattice-gas model of a graphite anode in a lithium-ion battery. Phys. Chem. Chem. Phys. 2017, 19, $23138-23145$.

(220) Gavilán-Arriazu, E.; Pinto, O.; López de Mishima, B.; Barraco, D.; Oviedo, O.; Leiva, E. The kinetic origin of the Daumas-Hérold model for the Li-ion/graphite intercalation system. Electrochemistry Communications 2018, 93, 133 - 137.

(221) Plett, G. L. Extended Kalman filtering for battery management systems of LiPB-based HEV battery packs: Part 2. Modeling and identification. Journal of Power Sources

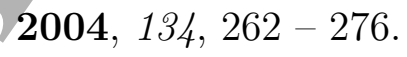


(222) Sole, C.; Drewett, N. E.; Hardwick, L. J. In situ Raman study of lithium-ion intercalation into microcrystalline graphite. Faraday Discussions 2014, 172, 223-237.

(223) Allart, D.; Montaru, M.; Gualous, H. Model of Lithium Intercalation into Graphite by Potentiometric Analysis with Equilibrium and Entropy Change Curves of Graphite Electrode. Journal of The Electrochemical Society 2018, 165, A380-A387.

(224) Markevich, E.; Levi, M. D.; Aurbach, D. New Insight into Studies of the Cycling Performance of Li-Graphite Electrodes. Journal of The Electrochemical Society 2005, 152, A778.

(225) Reynier, Y.; Yazami, R.; Fultz, B. The entropy and enthalpy of lithium intercalation into graphite. Journal of Power Sources 2003, 119-121, 850 - 855.

(226) Liu, H.; Strobridge, F. C.; Borkiewicz, O. J.; Wiaderek, K. M.; Chapman, K. W.; Chupas, P. J.; Grey, C. P. Capturing metastable structures during high-rate cycling of $\mathrm{LiFePO}_{4}$ nanoparticle electrodes. Science 2014, 344, 1252817-1-7.

(227) Orikasa, Y.; Maeda, T.; Koyama, Y.; Murayama, H.; Fukuda, K.; Tanida, H.; Arai, H.; Matsubara, E.; Uchimoto, Y.; Ogumi, Z. Direct Observation of a Metastable Crystal Phase of $\mathrm{Li}_{x} \mathrm{FePO}_{4}$ under Electrochemical Phase Transition. Journal of the American Chemical Society 2013, 135, 5497-5500.

(228) Gallagher, K. G.; Dees, D. W.; Jansen, A. N.; Abraham, D. P.; Kang, S.-H. A Volume Averaged Approach to the Numerical Modeling of Phase-Transition Intercalation Electrodes Presented for $\mathrm{Li}_{x} \mathrm{C}_{6}$. Journal of The Electrochemical Society 2012, 159, A2029-A2037.

(229) Grimsmann, F.; Gerbert, T.; Brauchle, F.; Gruhle, A.; Parisi, J.; Knipper, M. Hysteresis and current dependence of the graphite anode color in a lithium-ion cell and analysis of lithium plating at the cell edge. Journal of Energy Storage 2018, 15, 17 - 
3685

3686

3687

3688

3689

3690

3691

3692

3693

3694

3695

3696

3697

3698

3699

3700

3701

3702

3703

3704

3705

3706

3707

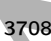

(230) Osswald, P. J.; del Rosario, M.; Garche, J.; Jossen, A.; Hoster, H. E. Fast and accurate measurement of entropy profiles of commercial lithium-ion cells. Electrochimica Acta 2015, 177, 270-276.

(231) Van der Ven, A.; Thomas, J.; Puchala, B.; Natarajan, A. First-Principles Statistical Mechanics of Multicomponent Crystals. Annual Review of Materials Research 2018, $48,27-55$.

(232) Lorie Lopez, J. L.; Grandinetti, P. J.; Co, A. C. Enhancing the real-time detection of phase changes in lithium-graphite intercalated compounds through derivative operando (dOp) NMR cyclic voltammetry. J. Mater. Chem. A 2018, 6, 231-243.

(233) Franco, A. A.; Rucci, A.; Brandell, D.; Frayret, C.; Gaberscek, M.; Jankowski, P.; Johansson, P. Boosting Rechargeable Batteries R\&D by Multiscale Modeling: Myth or Reality? Chemical Reviews 2019, 119, 4569-4627.

(234) Imai, Y.; Watanabe, A. Energetic evaluation of possible stacking structures of Liintercalation in graphite using a first-principle pseudopotential calculation. Journal of alloys and compounds 2007, 439, 258-267.

(235) Toyoura, K.; Koyama, Y.; Kuwabara, A.; Tanaka, I. Effects of off-stoichiometry of LiC $_{6}$ on the lithium diffusion mechanism and diffusivity by first principles calculations. The Journal of Physical Chèmistry C 2010, 114, 2375-2379.

(236) Yao, F.; Gunes, F.; Ta, H. Q.; Lee, S. M.; Chae, S. J.; Sheem, K. Y.; Cojocaru, C. S.; Xie, S. S.; Lee, Y. H. Diffusion mechanism of lithium ion through basal plane of layered graphene. Journal of the American Chemical Society 2012, 134, 8646-8654.

(237) NuLi, Y.; Yang, J.; Jiang, Z. Intercalation of lithium ions into bulk and powder highly oriented pyrolytic graphite. Journal of Physics and Chemistry of Solids 2006, 67, 882-886. 
3709

3710

3711

3712

3713

3714

3715

3716

3717

3718

3719

3720

3721

3722

3723

3724

3725

3726

3727

3728

3729

3730

3731

3732

(238) Liu, T.; Lin, L.; Bi, X.; Tian, L.; Yang, K.; Liu, J.; Li, M.; Chen, Z.; Lu, J.; Amine, K. et al. In situ quantification of interphasial chemistry in Li-ion battery. Nature nanotechnology 2019, 14, 50-56.

(239) Zhang, Z.; Smith, K.; Jervis, R.; Shearing, P. R.; Miller, T. S.; Brett, D. J. Operando Electrochemical Atomic Force Microscopy of Solid-Electrolyte-Interphase Formation on Graphite Anodes: The Evolution of SEI Morphology and Mechanical Properties. ACS applied materials \& interfaces 2020, 12, 35132-35141.

(240) Thinius, S.; Islam, M. M.; Bredow, T. Reconstruction of low-index graphite surfaces. Surface Science 2016, 649, 60-65.

(241) Larciprete, R.; Lacovig, P.; Gardonio, S.; Baraldi, A.; Lizzit, S. Atomic Oxygen on Graphite: Chemical Characterization and Thermal Reduction. The Journal of Physical Chemistry C 2012, 116, 9900-9908.

(242) Bernardo, P.; Le Meins, J.-M.; Vidal, L.; Dentzer, J.; Gadiou, R.; Märkle, W.; Novák, P.; Spahr, M. E.; Vix-Guterl, C. Influence of graphite edge crystallographic orientation on the first lithium intercalation in Li-ion battery. Carbon 2015, 91, 458467.

(243) Toyoura, K.; Koyama, Y.; Kuwabara, A.; Oba, F.; Tanaka, I. First-principles approach to chemical diffusion of lithium atoms in a graphite intercalation compound. Physical Review B 2008, 78, 214303.

(244) Takami, N.; Satoh, A.; Hara, M.; Ohsaki, T. Structural and kinetic characterization of lithium intercalation into carbon anodes for secondary lithium batteries. Journal of the Electrochemical Society 1995, 142, 371.

(245) Yang, H.; Bang, H. J.; Prakash, J. Evaluation of electrochemical interface area and lithium diffusion coefficient for a composite graphite anode. Journal of the Electro$3733 \quad$ chemical Society 2004, 151, A1247. 
3734

3735

3736

3737

3738

3739

3740

3741

3742

3743

3744

3745

3746

3747

3748

3749

3750

3751

3752

3753

3754

3755

$3756 \quad(255)$

(246) Yu, P.; Popov, B. N.; Ritter, J. A.; White, R. E. Determination of the lithium ion diffusion coefficient in graphite. Journal of The Electrochemical Society 1999, 146, 8.

(247) Uthaisar, C.; Barone, V. Edge effects on the characteristics of Li diffusion in graphene. Nano letters 2010, 10, 2838-2842.

(248) Leggesse, E. G.; Chen, C.-L.; Jiang, J.-C. Lithium diffusion in graphène and graphite: Effect of edge morphology. Carbon 2016, 103, 209-216.

(249) Chan, T.-L.; Chelikowsky, J. R. Controlling diffusion of lithium in silicon nanostructures. Nano letters 2010, 10, 821-825.

(250) Fujita, M.; Wakabayashi, K.; Nakada, K.; Kusakabe, K. Peculiar localized state at zigzag graphite edge. Journal of the Physical Society of Japan 1996, 65, 1920-1923.

(251) Lee, H.; Son, Y.-W.; Park, N.; Han, S.; Yu, J. Magnetic ordering at the edges of graphitic fragments: Magnetic tail interactions between the edge-localized states. Physical Review B 2005, 72, 174431.

(252) Velický, M.; Toth, P. S.; Woods, C. R.; Novoselov, K. S.; Dryfe, R. A. Electrochemistry of the Basal Plane versus Edge Plane of Graphite Revisited. The Journal of Physical Chemistry C 2019, 128, 11677-11685.

(253) Gerischer, H. An interpretation of the double layer capacity of graphite electrodes in relation to the density of states at the Fermi level. The Journal of Physical Chemistry $1985,89,4249-4251$.

(254) Weydanz, W.; Way, B.; Van Buuren, T.; Dahn, J. Behavior of Nitrogen-Substituted Carbon $\left(\mathrm{N}_{z} \mathrm{C}_{1-z}\right)$ in $\mathrm{Li} / \mathrm{Li}\left(\mathrm{N}_{z} \mathrm{C}+1-z\right)_{6}$ Cells. Journal of the Electrochemical Society $1994,141,900$. ${ }_{3757}$ dithium in $\mathrm{Li}_{x}\left(\mathrm{~B}_{z} \mathrm{C}_{1-z}\right)_{6}$. Journal of The Electrochemical Society 1994, 141, 907. 
(256) Endo, M.; Hayashi, T.; Hong, S.-H.; Enoki, T.; Dresselhaus, M. S. Scanning tunneling microscope study of boron-doped highly oriented pyrolytic graphite. Journal of Applied Physics 2001, 90, 5670-5674.

(257) Ishii, T.; Kaburagi, Y.; Yoshida, A.; Hishiyama, Y.; Oka, H.; Setoyama, N.; ichi Ozaki, J.; Kyotani, T. Analyses of trace amounts of edge sites in natural graphite, synthetic graphite and high-temperature treated coke for the understanding of their carbon molecular structures. Carbon 2017, 125, 146-155.

(258) Peng, C.; Bhandari, A.; Dziedzic, J.; Owen, J. R.; Skylaris, C.-K.; Kramer, D. Mechanism of Li nucleation at graphite anodes and mitigation strategies. J. Mater. Chem. A 2021, 9, 16798-16804.

(259) Gao, T.; Han, Y.; Fraggedakis, D.; Das, S.; Zhou, T.; Yeh, C. N.; Xu, S.; Chueh, W. C.; Li, J.; Bazant, M. Z. Interplay of Lithium Intercalation and Plating on a Single Graphite Particle. Joule 2021, 5, 393-414.

(260) Bhandari, A.; Peng, C.; Dziedzic, J.; Anton, L.; Owen, J. R.; Kramer, D.; Skylaris, C. K. Electrochemistry from first-principles in the grand canonical ensemble. Journal of Chemical Physics 2021, 155, 1-14.

(261) Winter, M. The solid electrolyte interphase-the most important and the least understood solid electrolyte in rechargeable Li batteries. Zeitschrift für physikalische Chemie 2009, 223, 1395-1406.

(262) Verma, P.; Maire, P.; Novák, P. A review of the features and analyses of the solid electrolyte interphase in Li-ion batteries. Electrochimica Acta 2010, 55, 6332 - 6341.

(263) Haruyama, J.; Ikeshoji, T.; Otani, M. Analysis of lithium insertion/desorption reaction at interfaces between graphite electrodes and electrolyte solution using density functional + implicit solvation theory. The Journal of Physical Chemistry C 2018, 3782 122, 9804-9810. 
3783

3784

3785

3786

3787

3788

3789

3790

3791

3792

3793

3794

3795

3796

3797

3798

3799

3800

3801

3802

3803

3804

3805

(264) Yamada, Y.; Iriyama, Y.; Abe, T.; Ogumi, Z. Kinetics of Lithium Ion Transfer at the Interface between Graphite and Liquid Electrolytes: Effects of Solvent and Surface Film. Langmuir 2009, 25, 12766-12770.

(265) Shi, S.; Lu, P.; Liu, Z.; Qi, Y.; Hector, L. G.; Li, H.; Harris, S. J. Direct calculation of Li-ion transport in the solid electrolyte interphase. Journal of the American Chemical Society 2012, 134, 15476-15487.

(266) Larcher, D.; Beattie, S.; Morcrette, M.; Edstroem, K.; Jumas, J.-C.; Tarascon, J.-M. Recent findings and prospects in the field of pure metals as negative electrodes for Li-ion batteries. Journal of Materials Chemistry 2007, 17, 3759-3772.

(267) Feng, K.; Li, M.; Liu, W.; Kashkooli, A. G.; Xiao, X.; Cai, M.; Chen, Z. Silicon-based anodes for lithium-ion batteries: from fundamentals to practical applications. Small 2018, 14, 1702737.

(268) Nitta, N.; Yushin, G. High-capacity anode materials for lithium-ion batteries: choice of elements and structures for active particles. Particle $\&$ Particle Systems Characterization 2014, 31, 317-336.

(269) Wagner, N. P.; Tron, A.; Tolchard, J. R.; Noia, G.; Bellmann, M. P. Silicon anodes for lithium-ion batteries produced from recovered kerf powders. Journal of Power Sources 2019, 414, 486-494.

(270) Okamoto, H. The Li-Si (Lithium-Silicon) system. Bulletin of Alloy Phase Diagrams 1990, 11,306-312.

(271) Gruber, T.; Thomas, D.; Röder, C.; Mertens, F.; Kortus, J. Raman spectroscopic studies of $\mathrm{Li}_{x} \mathrm{Si}_{y}$ compounds. Journal of Raman Spectroscopy 2013, 44, 934-938.

(272) Jiang, Y.; Offer, G.; Jiang, J.; Marinescu, M.; Wang, H. Voltage Hysteresis Model for Silicon Electrodes for Lithium Ion Batteries, Including Multi-Step Phase Transfor- 

mations, Crystallization and Amorphization. Journal of The Electrochemical Society 2020, 167, 130533 .

(273) Kuhn, A.; Sreeraj, P.; Pöttgen, R.; Wiemhöfer, H.-D.; Wilkening, M.; Heitjans, P. Li Ion Diffusion in the Anode Material $\mathrm{Li}_{12} \mathrm{Si}_{7}$ : Ultrafast Quasi-1D Diffusion and Two Distinct Fast 3D Jump Processes Separately Revealed by 7 Li NMR Relaxometry. Journal of the American Chemical Society 2011, 133, 11018-11021.

(274) Wang, D.; Gao, M.; Pan, H.; Wang, J.; Liu, Y. High performance amorphous-Si@ $\mathrm{SiOx} / \mathrm{C}$ composite anode materials for Li-ion batteries derived from ball-milling and in situ carbonization. Journal of Power Sources 2014, 256, 190-199.

(275) Kirklin, S.; Meredig, B.; Wolverton, C. High-Throughput Computational Screening of New Li-Ion Battery Anode Materials. Advanced Energy Materials 2013, 3, 252-262.

(276) Fang, C.; Wang, X.; Meng, Y. S. Key issues hindering a practical lithium-metal anode. Trends in Chemistry 2019, 1, 152-158.

(277) Li, S.; Jiang, M.; Xie, Y.; Xu, H.; Jia, J.; Li, J. Developing high-performance lithium metal anode in liquid electrolytes: challenges and progress. Advanced Materials 2018, 30, 1706375 .

(278) Radin, M. D.; Alvarado, J., Meng, Y. S.; Van der Ven, A. Role of Crystal Symmetry in the Reversibility of Stacking-Sequence Changes in Layered Intercalation Electrodes. Nano Letters 2017, 17, 7789-7795.

(279) Vinckeviciūtè, J.; Radin, M. D.; Van der Ven, A. Stacking-Sequence Changes and Na Ordering in Layered Intercalation Materials. Chemistry of Materials 2016, 28, $8640-8650$.

(280) Schmickler, W.; Santos, E. Interfacial electrochemistry, 2nd ed.; Springer-Verlag Berlin Heidelberg, 2010; pp 1-272. 
3831

3832

3833

3834

3835

3836

3837

3838

3839

3840

3841

3842

3843

3844

3845

3846

3847

3848

3849

3850

3851

3852

3853

(281) Bard, A. J.; Faulkner Larry R., Electrochemical Methods: Fundamentals and Applications, 2nd ed.; John Wiley \& Sons, Ltd, 2010; pp 1-864.

(282) Kralisch, D.; Stark, A.; Körsten, S.; Kreisel, G.; Ondruschka, B. Energetic, environmental and economic balances: Spice up your ionic liquid research efficiency. Green Chemistry 2005, 7, 301-309.

(283) Smiglak, M.; Reichert, W. M.; Holbrey, J. D.; Wilkes, J. S.; Sun, L.; Thrasher, J. S.; Kirichenko, K.; Singh, S.; Katritzky, A. R.; Rogers, R. D. Combustible ionic liquids by design: is laboratory safety another ionic liquid myth? Chemical Communications 2006, 2554-2556.

(284) Ding, F.; Xu, W.; Chen, X.; Zhang, J.; Engelhard, M. H.; Zhang, Y.; Johnson, B. R.; Crum, J. V.; Blake, T. A.; Liu, X., et al. Effects of carbonate solvents and lithium salts on morphology and coulombic efficiency of lithium electrode. Journal of The Electrochemical Society 2013, 160, A1894.

(285) Janek, J.; Zeier, W. G. A solid future for battery development. Nature Energy 2016, 1,16141 .

(286) Kazemiabnavi, S.; Zhang, Z.; Thornton, K.; Banerjee, S. Electrochemical stability window of imidazolium-based ionic liquids as electrolytes for lithium batteries. The Journal of Physical Chemistry B 2016, 120, 5691-5702.

(287) Park, M.; Zhang, X.; Chung, M.; Less, G. B.; Sastry, A. M. A review of conduction phenomena in Li-ion batteries. Journal of Power Sources 2010, 195, 7904-7929.

(288) Kamaya, N.; Homma, K.; Yamakawa, Y.; Hirayama, M.; Kanno, R.; Yonemura, M.; Kamiyama, T.; Kato, Y.; Hama, S.; Kawamoto, K.; Mitsui, A. A lithium superionic conductor. Nature materials 2011, 10, 682-6. 
(289) Swift, M. W.; Swift, J. W.; Qi, Y. Modeling the electrical double layer at solid-state electrochemical interfaces. Nature Computational Science 2021, 1, 212-220.

(290) Xu, K.; von Cresce, A. Interfacing electrolytes with electrodes in $\mathrm{Li}$ ion batteries. Journal of Materials Chemistry 2011, 21, 9849.

(291) Yu, C.; Ganapathy, S.; van Eck, E. R. H.; Wang, H.; Basak, S.; Li, Z., Wagemaker, M. Accessing the bottleneck in all-solid state batteries, lithium-ion transport over the solid-electrolyte-electrode interface. Nature Communications 2017, 8, 1086.

(292) Xu, K. Nonaqueous Liquid Electrolytes for Lithium-Based Rechargeable Batteries. Chemical Reviews 2004, 104, 4303-4418.

(293) Xu, K. Electrolytes and interphases in Li-ion patteries and beyond. Chemical Reviews 2014, 114, 11503-11618.

(294) Maier, J. Ionic conduction in space charge regions. Prog. Sol. Stat. Chem. 1995, 23, $171-263$.

(295) Mebane, D. S. A variational approach to surface cation segregation in mixed conducting perovskites. Comp. Mater. Sci. 2015, 103, $231-236$.

(296) Mebane, D. S.; De Souza, R. A. A generalised space-charge theory for extended defects in oxygen-ion conducting electrolytes: from dilute to concentrated solid solutions. Energy Environ. Sci. 2015, 8, 2935-2940.

(297) Tong, X.; Mebane, D, S.; De Souza, R. A. Analyzing the grain-boundary resistance of oxide-ion conducting electrolytes: Poisson-Cahn vs Poisson-Boltzmann theories. J. Amer. Ceram. Soc. 2020, 103, 5-22.

(298) Lund, J.; Vikrant, K.; Bishop, C.; Rheinheimer, W.; García, R. Thermodynamically consistent variational principles for charged interfaces. Acta Materialia 2021, 205, 
(299) Bazant, M. Z.; Storey, B. D.; Kornyshev, A. A. Double layer in ionic liquids: Overscreening versus crowding. Physical review letters 2011, 106, 046102.

(300) Perkin, S. Ionic liquids in confined geometries. Phys. Chem. Chem. Phys. 2012, 14, $5052-5062$.

(301) Groves, T. S.; Perez-Martinez, C. S.; Lhermerout, R.; Perkin, S. Surrface Forces and Structure in a Water-in-Salt Electrolyte. The Journal of Physical Chemistry Letters 2021, 12, 1702-1707.

(302) Bowers, J.; Vergara-Gutierrez, M. C.; Webster, J. R. P. Surface Ordering of Amphiphilic Ionic Liquids. Langmuir 2004, 20, 309-312.

(303) Sloutskin, E.; Ocko, B. M.; Tamam, L.; Kuzmenko, 1.; Gog, T.; Deutsch, M. Surface Layering in Ionic Liquids: An X-ray Reflectivity Study. Journal of the American Chemical Society 2005, 127, 7796-7804.

(304) Dean, J. M.; Coles, S. W.; Saunders, W. R.; McCluskey, A. R.; Wolf, M. J.; Walker, A. B.; Morgan, B. J. Overscreening and Underscreening in Solid-Electrolyte Grain Boundary Space-Charge Layers. Phys. Rev. Lett. 2021, 127, 135502.

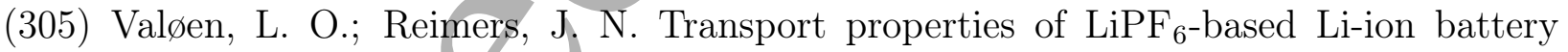
electrolytes. Journal of The Electrochemical Society 2005, 152, A882.

(306) Yamada, Y.; Yaegashi, M.; Abe, T.; Yamada, A. A superconcentrated ether electrolyte for fast-charging Li-ion batteries. Chem. Commun. 2013, 49, 11194.

(307) MacFarlane, D. R.; Tachikawa, N.; Forsyth, M.; Pringle, J. M.; Howlett, P. C.; Elliott, G. D.; Davis, J. H.; Watanabe, M.; Simon, P.; Angell, C. A. Energy applications of ionic liquids. Energy \& Environmental Science 2014, 7, 232-250.

308) Suo, L.; Borodin, O.; Sun, W.; Fan, X.; Yang, C.; Wang, F.; Gao, T.; Ma, Z.; Schroeder, M.; von Cresce, A., et al. Advanced high-voltage aqueous lithium-ion bat- 
3902

3903

3904

3905

3906

3907

3908

3909

3910

3911

3912

3913

3914

3915

3916

3917

3918

3919

3920

3921

3922

3923

3924

tery enabled by "water-in-bisalt" electrolyte. Angewandte Chemie 2016, 128, 72527257.

(309) Qian, J.; Henderson, W. A.; Xu, W.; Bhattacharya, P.; Engelhard, M.; Borodin, O.; Zhang, J.-G. High rate and stable cycling of lithium metal anode. Nature communications 2015, 6, 1-9.

(310) Kim, H.; Wu, F.; Lee, J. T.; Nitta, N.; Lin, H.-T.; Oschatz, M.; Cho, W. I.; Kaskel, S.; Borodin, O.; Yushin, G. In situ formation of protective coatings on sulfur cathodes in lithium batteries with LiFSI-based organic electrolytes. Advanced energy materials 2015, 5, 1401792 .

(311) Zhou, X.; Zhou, J.; Yin, Y. Modeling and Diagnostics of Polymer Electrolyte Fuel Cells; Springer, 2009; pp 307-380.

(312) Enderby, J.; Neilson, G. The structure of electrolyte solutions. Reports on Progress in Physics 1981, 44, 593.

(313) Fujie, T.; Takenaka, N.; Suzuki, Y.; Nagaoka, M. Red Moon methodology compatible with quantum mechanics/molecular mechanics framework: Application to solid electrolyte interphase film formation in lithium-ion battery system. The Journal of Chemical Physics 2018, 149, 044113.

(314) Koch, V. R.; Dominey, L. A.; Nanjundiah, C.; Ondrechen, M. J. The Intrinsic Anodic Stability of Several Anions Comprising Solvent-Free Ionic Liquids. Journal of The Electrochemical Society 1996, 143, 798-803.

(315) Ong, S. P.; Andreussi, O.; Wu, Y.; Marzari, N.; Ceder, G. Electrochemical windows of room-temperature ionic liquids from molecular dynamics and density functional theory calculations. Chemistry of Materials 2011, 23, 2979-2986. 
(316) Vuilleumier, R.; Sprik, M. Electronic properties of hard and soft ions in solution: Aqueous $\mathrm{Na}^{+}$and $\mathrm{Ag}^{+}$compared. The Journal of Chemical Physics 2001, 115, 34543468 .

(317) Blumberger, J.; Tavernelli, I.; Klein, M. L.; Sprik, M. Diabatic free energy curves and coordination fluctuations for the aqueous $\mathrm{Ag}^{+} / \mathrm{Ag}^{2+}$ redox couple: A biased Born-Oppenheimer molecular dynamics investigation. The Journal of chemical physics 2006, 124, 064507.

(318) Blumberger, J.; Bernasconi, L.; Tavernelli, I.; Vuilleumier, R.; Sprik, M. Electronic structure and solvation of copper and silver ions: a theoretical picture of a model aqueous redox reaction. Journal of the American Chemical Society 2004, 126, 39283938 .

(319) VandeVondele, J.; Sulpizi, M.; Sprik, M. From solvent fluctuations to quantitative redox properties of quinones in methanol and acetonitrile. Angewandte Chemie 2006, 118, 1970-1972.

(320) Lynden-Bell, R. Can Marcus theory be applied to redox processes in ionic liquids? A comparative simulation study of dimethylimidazolium liquids and acetonitrile. The Journal of Physical Chemistry B 2007, 111, 10800-10806.

(321) Ganesh, P.; Jiang, D.-E.; Kent, P. R. Accurate static and dynamic properties of liquid electrolytes for Li-ion batteries from ab initio molecular dynamics. Journal of Physical Chemistry B 2011, 115, 3085-3090.

(322) Kameda, Y.; Umebayashi, Y.; Takeuchi, M.; Wahab, M. A.; Fukuda, S.; Ishiguro, S. I.; Sasaki, M.; Amo, Y.; Usuki, T. Solvation structure of $\mathrm{Li}+$ in concentrated $\mathrm{LiPF}_{6^{-}}$ propylene carbonate solutions. Journal of Physical Chemistry B 2007, 111, 61046109 . 
(323) Soetens, J.-C.; Millot, C.; Maigret, B. Molecular Dynamics Simulation of $\mathrm{Li}^{+} \mathrm{BF}_{4}^{-}$ in Ethylene Carbonate, Propylene Carbonate, and Dimethyl Carbonate Solvents. J. Phys. Chem. A 1998, 102, 1055-1061.

(324) Lespes, N.; Filhol, J.-S. Using implicit solvent in ab initio electrochemical modeling: investigating $\mathrm{Li}^{+} / \mathrm{Li}$ electrochemistry at a $\mathrm{Li} /$ solvent interface. Journal of chemical theory and computation 2015, 11, 3375-3382.

(325) Morgan, B. J.; Watson, G. W. Role of Lithium Ordering in the $\mathrm{Li}_{x} \mathrm{TiO}_{2}$ Anatase $\rightarrow$ Titanate Phase Transition. J. Phys. Chem. Lett. 2011, 2, 1657-1661.

(326) Zhao, X.-G.; Dalpian, G. M.; Wang, Z.; Zunger, A. Polymorphous nature of cubic halide perovskites. Phys. Rev. B 2020, 101, 155137.

(327) Yeh, I.-C.; Hummer, G. System-Size Dependence of Diffusion Coefficients and Viscosities from Molecular Dynamics Simulations/with Periodic Boundary Conditions. J. Phys. Chem. B 2004, 108, 15873-15879.

(328) Botan, A.; Marry, V.; Rotenberg, B, Diffusion in bulk liquids: finite-size effects in anisotropic systems. Molecular Physics 2015, 113, 2674-2679.

(329) Horbach, J.; Kob, W.; Binder, K.; Angell, C. A. Finite size effects in simulations of glass dynamics. Phys. Rev. E 1996, 54, R5897-R5900.

(330) Coles, S. W.; Park, C.; Nikam, R.; Kanduč, M.; Dzubiella, J.; Rotenberg, B. Correlation Length in Concentrated Electrolytes: Insights from All-Atom Molecular Dynamics Simulations. J. Phys. Chem. B 2020, 124, 1778-1786.

(331) Frenkel, D.; Smit, B. Understanding molecular simulation; Academic Press, 2002.

(332) Lindahl,; Abraham,; Hess,; van der Spoel, GROMACS 2021 Manual. 2021,

(333) Canongia Lopes, J. N.; Pádua, A. A. H. CL\&P: A generic and systematic force field 
${ }_{3973}$ (334) Jorgensen, W. L.; Maxwell, D. S.; Tirado-Rives, J. Development and Testing of the

3974

3975

3976

3977

3978

3979

3980

3981

3982

3983

3984

3985

3986

3987

3988

3989

3990

3991

3992

3993

3994 OPLS All-Atom Force Field on Conformational Energetics and Properties of Organic Liquids. J. Am. Chem. Soc. 1996, 118, 11225-11236.

(335) Hess, B.; Bekker, H.; Berendsen, H. J. C.; Fraaije, J. G. E. M. LINCS: A linear constraint solver for molecular simulations. Journal of Computational Chemistry 1997, $18,1463-1472$.

(336) Ryckaert, J.-P.; Ciccotti, G.; Berendsen, H. J. C. Numerical integration of the cartesian equations of motion of a system with constraints: molecular dynamics of n-alkanes. Journal of Computational Physics 1977, 23, 327-341.

(337) Andersen, H. C. Rattle: A "velocity" version of the shake algorithm for molecular dynamics calculations. Journal of Computational Physics 1983, 52, 24-34.

(338) Ewald, P. P. Die Berechnung optischer und elektrostatischer Gitterpotentiale. Annalen der Physik 1921, 369, 253-287.

(339) Darden, T.; York, D.; Pedersen, L. Particle mesh Ewald: An N.log(N) method for Ewald sums in large systems. J. Chem. Phys. 1993, 98, 10089-10092.

(340) Deserno, M.; Holm, C. How to mesh up Ewald sums. II. An accurate error estimate for the particle-particle-particle-mesh algorithm. J. Chem. Phys. 1998, 109, 7694-7701.

(341) Yeh, I.-C.; Berkowitz, M. L. Ewald summation for systems with slab geometry. J. Chem. Phys. 1999, 111, 3155-3162.

(342) Canongia Lopes, J. N.; Deschamps, J.; Pádua, A. A. H. Modeling Ionic Liquids Using a Systematic All-Atom Force Field. J. Phys. Chem. B 2004, 108, 2038-2047.

(343) Canongia Lopes, J. N.; Pádua, A. A. H. Molecular Force Field for Ionic Liquids Composed of Triflate or Bistriflylimide Anions. J. Phys. Chem. B 2004, 108, 16893-16898. 
3996

3997

3998

3999

4000

4001

4002

4003

4004

4005

4006

4007

4008

4009

4010

4011

4012

4013

4014

4015

4016

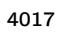

4018

(344) Canongia Lopes, J. N.; Pádua, A. A. H. Molecular Force Field for Ionic Liquids III: Imidazolium, Pyridinium, and Phosphonium Cations; Chloride, Bromide, and Dicyanamide Anions. J. Phys. Chem. B 2006, 110, 19586-19592.

(345) Spackman, M. A. Potential derived charges using a geodesic point selection scheme. Journal of Computational Chemistry 1996, 17, 1-18.

(346) Breneman, C. M.; Wiberg, K. B. Determining atom-centered monopoles from molecular electrostatic potentials. The need for high sampling density in formamide conformational analysis. Journal of Computational Chemistry 1990, 11, 361-373.

(347) Singh, U. C.; Kollman, P. A. An approach to computing electrostatic charges for molecules. Journal of Computational Chemistry 1984, 5, 129-145.

(348) Schröder, C. Comparing reduced partial charge models with polarizable simulations of ionic liquids. Phys. Chem. Chem. Phys. 2012, 14, 3089-3102.

(349) Schröder, C.; Lyons, A.; Rick, S. W. Polarizable MD simulations of ionic liquids: How does additional charge transfer change the dynamics? Phys. Chem. Chem. Phys. 2020, 22, 467-477.

(350) Shimizu, K.; Freitas, A. A.; Atkin, R.; Warr, G. G.; FitzGerald, P. A.; Doi, H.; Saito, S.; Ueno, K.; Umebayashi, Y.; Watanabe, M.; Lopes, J. N. C. Structural and aggregate analyses of ( $\mathrm{Li}$ salt + glyme) mixtures: the complex nature of solvate ionic liquids. Phys. Chem. Chem. Phys. 2015, 17, 22321-22335.

(351) Sangster, M.; Dixon, M. Interionic potentials in alkali halides and their use in simulations of the molten salts. Advances in Physics 1976, 25, 247-342.

(352) Madden, P. A.; Wilson, M. "Covalent" effects in "ionic" systems. Chem. Soc. Rev. 1996, 25, 339-350. 
4019

4020

4021

4022

4023

4024

4025

4026

4027

4028

4029

4030

4031

4032

4033

4034

4035

4036

4037

4038

4039

4040

4041

(353) Borodin, O. Polarizable Force Field Development and Molecular Dynamics Simulations of Ionic Liquids. J. Phys. Chem. B 2009, 113, 11463-11478.

(354) Borodin, O; Smith, G. D. LiTFSI Structure and Transport in Ethylene Carbonate from Molecular Dynamics Simulations. J. Phys. Chem. B 2006, 110, 4971-4977.

(355) Bedrov, D.; Piquemal, J.-P.; Borodin, O.; MacKerell, A. D.; Roux, B.; Schröder, C. Molecular Dynamics Simulations of Ionic Liquids and Electrolytes Using Polarizable Force Fields. Chem. Rev. 2019, 119, 7940-7995.

(356) Bedrov, D.; Borodin, O.; Li, Z.; Smith, G. D. Influence of Polarization on Structural, Thermodynamic, and Dynamic Properties of Ionic Liquids Obtained from Molecular Dynamics Simulations. J. Phys. Chem. B 2010, 114, 4984-4997.

(357) Marin-Laflèche, A.; Haefele, M.; Scalfi, L.; Coretti, A.; Dufils, T.; Jeanmairet, G.; Reed, S. K.; Alessandra, S.; Berthin, R.; Bacon, C.; Bonella, S.; Rotenberg, B.; Madden, P. A.; Salanne, M. MetalWalls: A classical molecular dynamics software dedicated to the simulation of electrochemical systems. Journal of Open Source Software 2020, 5, 2373.

(358) Mamatkulov, S.; Fyta, M.; Netz, R. R. Force fields for divalent cations based on single-ion and ion-pair properties. J. Chem. Phys. 2013, 138, 024505.

(359) Mamatkulov, S.; Schwierz, N. Force fields for monovalent and divalent metal cations in TIP3P water based on thermodynamic and kinetic properties. J. Chem. Phys. 2018, $148,074504$.

(360) Pethes, I. A comparison of classical interatomic potentials applied to highly concentrated aqueous lithium chloride solutions. Journal of Molecular Liquids 2017, 242, $845-858$. 
4042

4043

4044

4045

4046

4047

4048

4049

4050

4051

4052

4053

4054

4055

4056

4057

4058

4059

4060

4061

4062

4063

4064

(361) Hanke, C. G.; Price, S. L.; Lynden-Bell, R. M. Intermolecular potentials for simulations of liquid imidazolium salts. Molecular Physics 2001, 99, 801-809.

(362) Tsuzuki, S.; Shinoda, W.; Saito, H.; Mikami, M.; Tokuda, H.; Watanabe, M. Molecular Dynamics Simulations of Ionic Liquids: Cation and Anion Dependence of SelfDiffusion Coefficients of Ions. J. Phys. Chem. B 2009, 113, 10641-10649.

(363) Migliorati, V.; Serva, A.; Aquilanti, G.; Olivi, L.; Pasearelli, S.; Mathon, O.; D'Angelo, P. Combining EXAFS spectroscopy and molecular dynamics simulations to understand the structural and dynamic properties of an imidazolium iodide ionic liquid. Physical Chemistry Chemical Physics 2015, 17, 2464-2474.

(364) Chaudhari, M. I.; Muralidharan, A.; Pratt L. R.; Rempe, S. B. Assessment of Simple Models for Molecular Simulation of Ethylene Carbonate and Propylene Carbonate as Solvents for Electrolyte Solutions. Top Curr Chem (Z) 2018, 376, 376.

(365) Smith, A. M.; Lee, A. A.; Perkin, S. The Electrostatic Screening Length in Concentrated Electrolytes Increases with Concentration. J. Phys. Chem. Lett. 2016, 7, $2157-2163$.

(366) Gaddam, P.; Ducker, W. Electrostatic Screening Length in Concentrated Salt Solutions. Langmuir 2019, 35, 5719-5727.

(367) Zeman, J.; Kondrat, S.; Holm, C. Bulk ionic screening lengths from extremely largescale molecular dynamics simulations. Chem. Commun. 2020, 56, 15635-15638.

(368) Krucker-Velasquez, E.; Swan, J. W. Underscreening and hidden ion structures in large scale simulations of concentrated electrolytes. The Journal of Chemical Physics 2021, $155,134903$.

(369) Jeanmairet, G.; Rotenberg, B.; Borgis, D.; Salanne, M. Study of a water-graphene 

capacitor with molecular density functional theory. The Journal of chemical physics 2019, 151, 124111.

(370) Cats, P.; Evans, R.; Härtel, A.; van Roij, R. Primitive model electrolytes in the near and far field: Decay lengths from DFT and simulations. The Journal of Chemical Physics 2021, 154, 124504.

(371) Merlet, C.; Péan, C.; Rotenberg, B.; Madden, P. A.; Daffos, B.; Táberna, P.-L.; Simon, P.; Salanne, M. Highly confined ions store charge more efficiently in supercapacitors. Nature Communications 2013, 4, 2701.

(372) Coles, S. W.; Mishin, M.; Perkin, S.; V. Fedorov, M.; B. Ivaništšev, V. The nanostructure of a lithium glyme solvate ionic liquid at electrified interfaces. Physical Chemistry Chemical Physics 2017, 19, 11004-11010.

(373) Coles, S. W.; Ivaništšev, V. B. Simulation of a Solvate Ionic Liquid at a Polarizable Electrode with a Constant Potential. J. Phys. Chem. C 2019, 123, 3935-3943.

(374) Sergeev, A. V.; Chertovich, A. V.; Itkis, D. M.; Sen, A.; Gross, A.; Khokhlov, A. R. Electrode/Electrolyte Interface in/ the Li- $\mathrm{O}_{2}$ Battery: Insight from Molecular Dynamics Study. J. Phys. Chem. C 2017, 121, 14463-14469.

(375) Schröder, C. Collective translational motions and cage relaxations in molecular ionic liquids. J. Chem. Phys. 2011, 135, 024502.

(376) Schröder, C.; Steinhauser, O. On the dielectric conductivity of molecular ionic liquids. J. Chem. Phys. 2009, 131, 114504.

(377) Hayamizu, K.; Aihara, Y.; Arai, S.; Martinez, C. G. Pulse-gradient spin-echo ${ }^{1} \mathrm{H},{ }^{7} \mathrm{Li}$, and ${ }^{1} 9 \mathrm{~F}$ NMR diffusion and ionic conductivity measurements of 14 organic electrolytes containing $\mathrm{LiN}\left(\mathrm{SO}_{2} \mathrm{CF}_{3}\right)_{2}$. Journal of Physical Chemistry B 1999, 103, 519-524. 
4088

4089

4090

4091

4092

4093

4094

4095

4096

4097

4098

4099

4100

4101

4102

4103

4104

4105

4106

4107

4108

4109

4110

(378) Lesch, V.; Li, Z.; Bedrov, D.; Borodin, O.; Heuer, A. The influence of cations on lithium ion coordination and transport in ionic liquid electrolytes: a MD simulation study. Phys. Chem. Chem. Phys. 2015, 18, 382-392.

(379) Borodin, O.; Smith, G. D.; Henderson, W. Li ${ }^{+}$Cation Environment, Transport, and Mechanical Properties of the LiTFSI Doped N-Methyl-N-alkylpyrrolidinium ${ }^{+} \mathrm{TFSI}^{-}$ Ionic Liquids. J. Phys. Chem. B 2006, 110, 16879-16886.

(380) Borodin, O.; Smith, G. D. Li ${ }^{+}$Transport Mechanism in Oligo(Ethylene Oxide)s Compared to Carbonates. J Solution Chem 2007, 36, 803-813.

(381) Onsager, L.; Kim, S. K. Wien Effect in Simple Strong Electrolytes. The Journal of Physical Chemistry 1957, 61, 198-215.

(382) Lesnicki, D.; Gao, C. Y.; Rotenberg, B.; Limmer, D. T. Field-Dependent Ionic Conductivities from Generalized Fluctuation-Dissipation Relations. Phys. Rev. Lett. 2020, 124, 206001.

(383) Jones, P.; Coupette, F; Härtel, A.; Lee, A. A. Bayesian unsupervised learning reveals hidden structure in concentrated êlectrolytes. The Journal of Chemical Physics 2021, 154, 134902.

(384) Skarmoutsos, I.; Ponnuchamy, V.; Vetere, V.; Mossa, S. Li + Solvation in Pure, Binary, and Ternary Mixtures of Organic Carbonate Electrolytes. J. Phys. Chem. C 2015, $119,4502-4515$.

(385) Takeuchi, M.; Matubayasi, N.; Kameda, Y.; Minofar, B.; ichi Ishiguro, S.; Umebayashi, Y. Free-Energy and Structural Analysis of Ion Solvation and Contact Ion-Pair Formation of $\mathrm{Li}^{+}$with $\mathrm{BF}_{4}^{-}$and $\mathrm{PF}_{6}^{-}$in Water and Carbonate Solvents. J. Phys. Chem. B 2012, 116, 6476-6487. 
4111 (386) Lazaridis, T. Inhomogeneous Fluid Approach to Solvation Thermodynamics, 1. The$4112 \quad$ ory. J. Phys. Chem. B 1998, 102, 3531-3541.

${ }_{4113}$ (387) Hall, D. S.; Self, J.; Dahn, J. R. Dielectric Constants for Quantum Chemistry and ${ }_{4114}$ Li-Ion Batteries: Solvent Blends of Ethylene Carbonate and Ethyl Methyl Carbonate.

(394) Perkin, S.; Chai, L.; Kampf, N.; Raviv, U.; Briscoe, W.; Dunlop, I.; Titmuss, S.; Seo, M.; Kumacheva, E.; Klein, J. Forces between Mica Surfaces, Prepared in Different 
${ }_{4135}$ Ways, Across Aqueous and Nonaqueous Liquids Confined to Molecularly Thịn Films.

4136

4137

4138

4139

4140

4141

4142

4143

4144

4145

4146

4147

4148

4149

4150

4151

4152

4153

4154

4155

4156

4157

4158

(402) Simoncelli, M.; Ganfoud, N.; Sene, A.; Haefele, M.; Daffos, B.; Taberna, P.-L.; Salanne, M.; Simon, P.; Rotenberg, B. Blue Energy and Desalination with Nanoporous

Langmuir 2006, 22, 6142-6152.

(395) Scalfi, L.; Dufils, T.; Reeves, K. G.; Rotenberg, B.; Salanne, M. A semiclassical Thomas-Fermi model to tune the metallicity of electrodes in molecular simulations. J. Chem. Phys. 2020, 153, 174704.

(396) Vatamanu, J.; Borodin, O. Ramifications of Water-in-Salt Interfacial Structure at Charged Electrodes for Electrolyte Electrochemical Stability. J. Phys. Chem. Lett. 2017, 8, 4362-4367.

(397) Li, Z.; Jeanmairet, G.; Méndez-Morales, T.; Rotenberg, B.; Salanne, M. Capacitive Performance of Water-in-Salt Electrolytes in Supercapacitors: A Simulation Study. J. Phys. Chem. C 2018, 122, 23917-23924.

(398) Vatamanu, J.; Borodin, O.; Smith, G. D. Molecular dynamics simulations of atomically flat and nanoporous electrodes with a molten salt electrolyte. 2009, 12, 170-182.

(399) Merlet, C.; Rotenberg, B.; Madden, P. A.; Taberna, P.-L.; Simon, P.; Gogotsi, Y.; Salanne, M. On the molecular origin of supercapacitance in nanoporous carbon electrodes. Nature Materials 2012, 11, 306-310.

(400) Vatamanu, J.; Cao, L.; Borodin, O.; Bedrov, D.; Smith, G. D. On the Influence of Surface Topography on the Electric Double Layer Structure and Differential Capacitance of Graphite/Ionic Liquid Interfaces. J. Phys. Chem. Lett. 2011, 2, 2267-2272.

(401) Borodin, O.; Bedrov, D. Interfacial Structure and Dynamics of the Lithium Alkyl Dicarbonate SEI Components in Contact with the Lithium Battery Electrolyte. J. Phys. Chem. C 2014, 118, 18362-18371. 

Carbon Electrodes: Capacitance from Molecular Simulations to Continuous, Models. Phys. Rev. X 2018, 8, 021024.

(403) Suo, L.; Borodin, O.; Gao, T.; Olguin, M.; Ho, J.; Fan, X.; Luo, C.; Wang, C.; Xu, K. "Water-in-salt" electrolyte enables high-voltage aqueous lithium-ion chemiștries. Science 2015, 350, 938-943.

(404) Chen, M.; Feng, G.; Qiao, R. Water-in-salt electrolytes: An interfacial perspective. Current Opinion in Colloid $\mathscr{G}$ Interface Science 2020, 47, 99-110.

(405) Li, Z.; Bouchal, R.; Mendez-Morales, T.; Rollet, A.-L.; Rizzi, C.; Le Vot, S.; Favier, F.; Rotenberg, B.; Borodin, O.; Fontaine, O., et al. Transport properties of Li-TFSI waterin-salt electrolytes. The Journal of Physical Chemistry B 2019, 123, 10514-10521.

(406) McCurry, J. Samsung blames two separate battery faults for Galaxy Note 7 fires. 2017; http://www.theguardian.com/technology/2017/jan/23/ samsung-blames-faulty-batteries-for-causing-galaxy-note-7-fires

(407) Zhang, S. S. Liquid eleetrolyte lithium/sulfur battery: Fundamental chemistry, problems, and solutions. Journal of Power Sources 2013, 231, 153-162.

(408) Wu, X.; Pan, K.; Jia, M.; Ren, Y.; He, H.; Zhang, L.; Zhang, S. Electrolyte for lithium protection: From liquid to solid. Green Energy and Environment 2019, 4, 360-374.

(409) Kushima, A.; So, K. P.; Su, C.; Bai, P.; Kuriyama, N.; Maebashi, T.; Fujiwara, Y.; Bazant, M. Z.; L1, J. Liquid cell transmission electron microscopy observation of lithium metal growth and dissolution: Root growth, dead lithium and lithium flotsams. Nano Energy 2017, 32, 271-279.

(410) Lin, D.; Liu, Y.; Li, Y.; Li, Y.; Pei, A.; Xie, J.; Huang, W.; Cui, Y. Fast galvanic lithium corrosion involving a Kirkendall-type mechanism. Nature chemistry 2019, 11, $382-389$. 


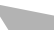

(411) Hallinan Jr, D. T.; Balsara, N. P. Polymer electrolytes. Annual review of materials research 2013, 43, 503-525.

(412) Popovic, J. Chemistry of Soft Matter Battery Electrolytes. Encyclopedia of Inorganic and Bioinorganic Chemistry 2011, 1-11.

(413) Shao, Y.; Knijff, L.; Dietrich, F. M.; Hermansson, K.; Zhang, C. Modelling Bulk Electrolytes and Electrolyte Interfaces with Atomistic Machine Learning. Batteries \& Supercaps 2021, 4, 585-595.

(414) Hellström, M.; Behler, J. Concentration-dependent proton transfer mechanisms in aqueous $\mathrm{NaOH}$ solutions: From acceptor-driven to donor-driven and back. The journal of physical chemistry letters 2016, 7, 3302-3306.

(415) Tovey, S.; Narayanan Krishnamoorthy, A.; Sivaraman, G.; Guo, J.; Benmore, C.; Heuer, A.; Holm, C. DFT Accurate Interatomic Potential for Molten NaCl from Machine Learning. The Journal of Physical Chemistry C 2020, 124, 25760-25768.

(416) Fedkin, M. V.; Shin, Y. K.; Dasgupta, N.; Yeon, J.; Zhang, W.; Van Duin, D.; Van Duin, A. C.; Mori, K.; Fujiwara, A.; Machida, M., et al. Development of the ReaxFF Methodology for Electrolyte-Water Systems. The Journal of Physical Chemistry A 2019, 123, 2125-2141.

(417) Hossain, M. J.; Pawar, G.; Liaw, B.; Gering, K. L.; Dufek, E. J.; van Duin, A. C. Lithium-electrolyte solvation and reaction in the electrolyte of a lithium ion battery: A ReaxFF reactive force field study. The Journal of chemical physics 2020, 152, 184301.

(418) Forsman, J.; Woodward, C. E.; Trulsson, M. A Classical Density Functional Theory of Ionic Liquids. J. Phys. Chem. B 2011, 115, 4606-4612. 
4206

4207

4208

4209

4210

4211

4212

4213

4214

4215

4216

4217

4218

4219

4220

4221

4222

4223

4224

4225

4226

4227

4228

4229

(419) Jeanmairet, G.; Rotenberg, B.; Levesque, M.; Borgis, D.; Salanne, M. A molecular density functional theory approach to electron transfer reactions. Chemical science 2019, 10, 2130-2143.

(420) Jie, Y.; Ren, X.; Cao, R.; Cai, W.; Jiao, S. Advanced liquid electrolytes for rechargeable Li metal batteries. Advanced Functional Materials 2020, 30, 1910777.

(421) Francis, C. F.; Kyratzis, I. L.; Best, A. S. Lithium-Ion Battery Separators for IonicLiquid Electrolytes: A Review. Advanced Materials 2020, 32, 1904205.

(422) Xiong, S.; Regula, M.; Wang, D.; Song, J. Toward better lithium-sulfur batteries: functional non-aqueous liquid electrolytes. Electrochemical Energy Reviews 2018, 1, $388-402$.

(423) Culver, S. P.; Koerver, R.; Krauskopf, T.; Zeier, W. G. Designing Ionic Conductors: The Interplay between Structural Phenomena and Interfaces in Thiophosphate-Based Solid-State Batteries. Chemistry of Materials 2018, 30, 4179-4192.

(424) Famprikis, T.; Canepa,P.; Dawson, J. A.; Islam, M. S.; Masquelier, C. Fundamentals of inorganic solid-state electrolytes for batteries. Nature Materials 2019, 18, 12781291.

(425) Goodenough, J. B.; Park, K.-S. The Li-Ion Rechargeable Battery: A Perspective. Journal of the American Chemical Society 2013, 135, 1167-1176.

(426) Dirican, M.; Yan, C.; Zhu, P.; Zhang, X. Composite solid electrolytes for all-solid-state lithium batteries. Materials Science and Engineering: R: Reports 2019, 136, 27 - 46.

(427) Han, L.; Lehmann, M. L.; Zhu, J.; Liu, T.; Zhou, Z.; Tang, X.; Heish, C.-T.; Sokolov, A. P.; Cao, P.; Chen, X. C., et al. Recent Developments and Challenges in Hybrid Solid Electrolytes for Lithium-Ion Batteries. Frontiers in Energy Research 2020, 8, 202. 
4230

4231

4232

4233

4234

4235

4236

4237

4238

4239

4240

4241

4242

4243

4245

4246

4247

4248

4249

4250

4251

(428) Manthiram, A.; Yu, X.; Wang, S. Lithium battery chemistries enabled by solid-state electrolytes. Nature Reviews Materials 2017, 2, 1-16.

(429) Faraday, M. IV. Experimental researches in electricity.-Third series. Philosophical Transactions of the Royal Society of London 1833, 123, 23-54.

(430) Armand, M.; Tarascon, J.-M. Building better batteries. Nature 2008, 451, 652-657.

(431) Sudworth, J. The sodium/nickel chloride (ZEBRA) battery. Journal of Power Sources 2001, 100, $149-163$.

(432) Oshima, T.; Kajita, M.; Okuno, A. Development of sodium-sulfur batteries. International Journal of Applied Ceramic Technology 2004, 1, 269-276.

(433) Capasso, C.; Veneri, O. Experimental analysis of a zebra battery based propulsion system for urban bus under dynamic conditions. Energy Procedia 2014, 61, 11381141.

(434) Dudney, N.; Bates, J.; Zuhr, R.; Luck, C.; Robertson, J. Sputtering of lithium compounds for preparation of electrolyte thin films. solid state ionics 1992, 53, 655-661.

(435) Bates, J.; Dudney, N.; Gruzalski, G.; Zuhr, R.; Choudhury, A.; Luck, C.; Robertson, J. Electrical properties of amorphous lithium electrolyte thin films. Solid state ionics 1992, 53, 647-654.

(436) Inaguma, Y.; Liquan, C.; Itoh, M.; Nakamura, T.; Uchida, T.; Ikuta, H.; Wakihara, M. High ionic conductivity in lithium lanthanum titanate. Solid State Communications 1993, 86, $689-693$.

(437) Kasper, H. Series of rare earth garnets $\mathrm{Ln}_{3}^{3+} \mathrm{M}_{2} \mathrm{Li}_{3}^{+} \mathrm{O}_{12}(\mathrm{M}=\mathrm{Te}, \mathrm{W})$. Inorganic Chemistry 1969, 8, 1000-1002.

(438) Mazza, D. Remarks on a ternary phase in the $\mathrm{La}_{2} \mathrm{O}_{3} \square \mathrm{Me}_{2} \mathrm{O}_{5} \square \mathrm{Li}_{2} \mathrm{O}$ system ( $\mathrm{Me}=\mathrm{Nb}$, ${ }_{4253}^{2}$ Ta). Materials Letters 1988, 7, 205-207. 
4254

4255

4256

4257

4258

4259

4260

4261

4262

4263

4264

4265

4266

4267

4268

4269

4270

4271

4272

4273

4274

4275

4276

(439) Kennedy, J. H.; Sahami, S.; Shea, S. W.; Zhang, Z. Preparation and conductivity measurements of $\mathrm{SiS}_{2} \square \mathrm{Li}_{2} \mathrm{~S}$ glasses doped with LiBr and LiCl. Solid State Ionics 1986, $18,368-371$.

(440) Ivanov-Shits, A.; Sigaryov, S. Ionic conductivity in LISICON-type materials. Solid state ionics 1988, 27, 89-100.

(441) Lang, B.; Ziebarth, B.; Elsässer, C. Lithium ion conduction in $\mathrm{LiTi}_{2}\left(\mathrm{PO}_{4}\right)_{3}$ and related compounds based on the NASICON structure: a first-principles study. Chemistry of Materials 2015, 27, 5040-5048.

(442) De Klerk, N. J.; Rosłoń, I.; Wagemaker, M. Diffusion/mechanism of Li argyrodite solid electrolytes for Li-ion batteries and prediction of optimized halogen doping: the effect of Li vacancies, halogens, and halogen disorder. Chemistry of Materials 2016, 28, 7955-7963.

(443) Dawson, J. A.; Attari, T. S., Chen, H.; Emge, S. P.; Johnston, K. E.; Islam, M. S. Elucidating lithium-ion and proton dynamics in anti-perovskite solid electrolytes. Energy E) Environmental Science 2018, 11, 2993-3002.

(444) Ahiavi, E.; Dawson, J.A.; Kudu, U.; Courty, M.; Islam, M. S.; Clemens, O.; Masquelier, C.; Famprikis, T. Mechanochemical synthesis and ion transport properties of $\mathrm{Na}_{3} \mathrm{OX}\left(\mathrm{X}=\mathrm{Cl}, \mathrm{Br}, \mathrm{I}\right.$ and $\left.\mathrm{BH}_{4}\right)$ antiperovskite solid electrolytes. Journal of Power Sources 2020, 471, 228489.

(445) Fenton, D. Complexes of alkali metal ions with poly (ethylene oxide). Polymer 1973, 14,589

(446) Abraham, K.; Alamgir, M. Li ${ }^{+}$-conductive solid polymer electrolytes with liquid-like conductivity. J. Electrochem. Soc 1990, 137, 1657. 
${ }_{4277}$ (447) Dautzenberg, G.; Croce, F.; Passerini, S.; Scrosati, B. Characterization of PAN-based

4278

4279

4280

4281

4282

4283

4284

4285

4286

4287

4288

4289

4290

4291

4292

4293

4294

4295

4296

4297

4298

4299 gel electrolytes. electrochemical stability and lithium cyclability. Chemistry of materials 1994, 6, 538-542.

(448) Arcella, V.; Sanguineti, A.; Quartarone, E.; Mustarelli, P. Vinylidenefluoridehexafluoropropylene copolymers as hybrid electrolyte components for lithium batteries. Journal of power sources 1999, 81, 790-794.

(449) Kataoka, H.; Saito, Y.; Sakai, T.; Quartarone, E.; Mustarelli, P. Conduction mechanisms of PVDF-type gel polymer electrolytes of lithium prepared by a phase inversion process. The Journal of Physical Chemistry B 2000, 104, 11460-11464.

(450) Li, W.; Zhu, Z.; Shen, W.; Tang, J.; Yang, G.; Xu, Z./A novel PVdF-based composite gel polymer electrolyte doped with ionomer modified graphene oxide. RSC Advances 2016, 6, 97338-97345.

(451) Appetecchi, G.; Croce, F.; Scrosati, B. Kinetics and stability of the lithium electrode in poly (methylmethacrylate)-based gel electrolytes. Electrochimica Acta 1995, 40, 991-997.

(452) Bohnke, O.; Frand, G.; Rezrazi, M.; Rousselot, C.; Truche, C. Fast ion transport in new lithium electrolytes gelled with PMMA. 1. Influence of polymer concentration. Solid State Ionics 1993, 66, 97-104.

(453) Abbrent, S.; Plestil, J.; Hlavata, D.; Lindgren, J.; Tegenfeldt, J.; Wendsjö, A. Crystallinity and morphology of PVdF-HFP-based gel electrolytes. Polymer 2001, 42, $1407-1416$.

(454) Park, M.-S.; Hyun, S.-H.; Nam, S.-C.; Cho, S. B. Performance evaluation of printed $\mathrm{LiCOO}_{2}$ cathodes with PVDF-HFP gel electrolyte for lithium ion microbatteries. Electrochimica Acta 2008, 53, 5523-5527. 
4301

4302

4303

4304

4305

4306

4307

4308

4309

4310

4311

4312

4313

4314

4315

4316

4317

4318

4319

4320

4321

4322

4323

(455) Yang, C.-C.; Lian, Z.-Y.; Lin, S.; Shih, J.-Y.; Chen, W.-H. Preparation and application of PVDF-HFP composite polymer electrolytes in $\mathrm{LiNi}_{0.5} \mathrm{Co}_{0.2} \mathrm{Mn}_{0.3} \mathrm{O}_{2}$ lithium-polymer batteries. Electrochimica Acta 2014, 134, 258-265.

(456) Zhou, W.; Wang, S.; Li, Y.; Xin, S.; Manthiram, A.; Goodenough, J. B.Plating a dendrite-free lithium anode with a polymer/ceramic/polymer sandwich electrolyte. Journal of the American Chemical Society 2016, 138, 9385-9388.

(457) Zhang, T.; He, W.; Zhang, W.; Wang, T.; Li, P.; Sun, Z.; Yu, X. Designing composite solid-state electrolytes for high performance lithium ion or lithium metal batteries. Chem. Sci. 2020, 11, 8686-8707.

(458) Uvarov, N. Composite solid electrolytes: recent advances and design strategies. Journal of Solid State Electrochemistry 2011, 15, 367-389.

(459) Wilkening, M.; Indris, S.; Heitjans, P. Heterogeneous lithium diffusion in nanocrystalline $\mathrm{Li}_{2} \mathrm{O}: \mathrm{Al}_{2} \mathrm{O}_{3}$ composites. Phys. Chem. Chem. Phys. 2003, 5, 2225-2231.

(460) Heitjans, P.; Indris, S. Diffusion and ionic conduction in nanocrystalline ceramics. Journal of Physics: Condensed Matter 2003, 15, R1257-R1289.

(461) Indris, S.; Heitjans, P.; Roman, H. E.; Bunde, A. Nanocrystalline versus Microcrystalline $\mathrm{Li}_{2} \mathrm{O}: \mathrm{B}_{2} \mathrm{O}_{3}$ Composites: Anomalous Ionic Conductivities and Percolation Theory. Phys. Rev. Lett. 2000, 84, 2889-2892.

(462) Indris, S.; Heitjans, P. Heterogeneous ${ }^{7} \mathrm{Li}$ NMR relaxation in nanocrystalline $\mathrm{Li}_{2} \mathrm{O}: \mathrm{B}_{2} \mathrm{O}_{3}$ composites. Journal of Non-Crystalline Solids 2002, 307-310, 555 - 564 .

(463) Blanchard, D.; Nale, A.; Sveinbjörnsson, D.; Eggenhuisen, T. M.; Verkuijlen, M. H.; Vegge, T.; Kentgens, A. P.; de Jongh, P. E. Nanoconfined $\mathrm{LiBH}_{4}$ as a fast lithium ion conductor. Advanced Functional Materials 2015, 25, 184-192. 
(464) Liang, C. Conduction characteristics of the lithium iodide-aluminum oxide solid electrolytes. Journal of the Electrochemical Society 1973, 120, 1289.

(465) Phipps, J.; Whitmore, D. Ion transport in $\mathrm{LiSiO}_{2}$ composites. Solid State Ionics 1983, 9, 123-130.

(466) Uvarov, N.; Isupov, V.; Sharma, V.; Shukla, A. Effect of morphology and particle size on the ionic conductivities of composite solid electrolytes. Solid State Ionics 1992, 51, $41-52$.

(467) Pradel, A.; Ribes, M. Electrical properties of lithium conductive silicon sulfide glasses prepared by twin roller quenching. Solid State Ionics 1986, 18, 351-355.

(468) Kanno, R. Synthesis of a new lithium ionic conductor, thio-LISICON-lithium germanium sulfide system. Solid State Ionics 2000, 130, 97-104.

(469) Murayama, M. Synthesis of New Lithium Ionic Conductor Thio-LISICON-Lithium Silicon Sulfides System. Journal of Solid State Chemistry 2002, 168, 140-148.

(470) Murayama, M.; Sonoyama, N.; Yamada, A.; Kanno, R. Material design of new lithium ionic conductor, thio-LISICON, in the $\mathrm{Li}_{2} \mathrm{~S}-\mathrm{P}_{2} \mathrm{~S}_{5}$ system. Solid State Ionics 2004, 170, 173-180.

(471) Minafra, N.; Culver, S. P.; Li, C.; Senyshyn, A.; Zeier, W. G. Influence of the Lithium Substructure on the Diffusion Pathways and Transport Properties of the Thio-LISICON Li $\mathrm{Ge}_{1}{ }_{x} \mathrm{Sn}_{x} \mathrm{~S}_{4}$. Chemistry of Materials 2019, 31, 3794-3802.

(472) Bron, P.; Johansson, S.; Zick, K.; Schmedt auf der Günne, J.; Dehnen, S.; Roling, B. $\mathrm{Li}_{10} \mathrm{SnP}_{2} \mathrm{~S}_{12}$ : An Affordable Lithium Superionic Conductor. Journal of the American Chemical Society 2013, 135, 15694-15697.

(473) Whiteley, J. M.; Woo, J. H.; Hu, E.; Nam, K.-W.; Lee, S.-H. Empowering the Lithium 

Metal Battery through a Silicon-Based Superionic Conductor. Journal of The Electrochemical Society 2014, 161, A1812-A1817.

(474) Huang, W.; Yoshino, K.; Hori, S.; Suzuki, K.; Yonemura, M.; Hirayama, M.; Kanno, R. Superionic lithium conductor with a cubic argyrodite-type structure in the Li-Al-Si-S system. Journal of Solid State Chemistry 2019, 270, 487-492.

(475) Yamane, H.; Shibata, M.; Shimane, Y.; Junke, T.; Seino, Y.; Adams, S.; Minami, K.; Hayashi, A.; Tatsumisago, M. Crystal structure of a superionic conductor, $\mathrm{Li}_{7} \mathrm{P}_{3} \mathrm{~S}_{11}$. Solid State Ionics 2007, 178, 1163-1167.

(476) Homma, K.; Yonemura, M.; Kobayashi, T.; Nagao, M.; Hirayama, M.; Kanno, R. Crystal structure and phase transitions of the lithium ionic conductor $\mathrm{Li}_{3} \mathrm{PS}_{4}$. Solid State Ionics 2011, 182, 53-58.

(477) Minami, T.; Hayashi, A.; Tatsumisago, M. Recent progress of glass and glass-ceramics as solid electrolytes for lithium secondary batteries. Solid State Ionics 2006, 177, 2715-2720.

(478) Bai, X.; Duan, Y.; Zhuang, W.; Yâng, R.; Wang, J. Research progress in Li-argyroditebased solid-state electrolytes. Journal of Materials Chemistry A 2020, 8, 25663-25686.

(479) Minafra, N.; Hogrefe, K.; Barbon, F.; Helm, B.; Li, C.; Wilkening, H. M. R.; Zeier, W. G. Two-Dimensional Substitution: Toward a Better Understanding of the Structure-Transport Correlations in the Li-Superionic Thio-LISICONs. Chemistry of Materials 2021, 33, 727-740.

(480) Bhandari, A.; Bhattacharya, J. Origin of Fast Ion Conduction in $L i_{10} G e P_{2} S_{12}$, a Superionic Conductor. Journal of Physical Chemistry C 2016, 120.

(481) Mo, Y.; Ong, S. P.; Ceder, G. First Principles Study of the $\mathrm{Li}_{10} \mathrm{GeP}_{2} \mathrm{~S}_{12}$ Lithium Super Ionic Conductor Material. Chemistry of Materials 2012, 24, 15-17. 
${ }_{4371}$ (482) Kraft, M. A.; Ohno, S.; Zinkevich, T.; Koerver, R.; Culver, S. P.; Fuchs, T.;

4372

4373

4374

4375

4376

4377

4378

4379

4380

4381

4382

4383

4384

4385

4386

4387

4388

4389

4390

4391

4392

4393

4394 Senyshyn, A.; Indris, S.; Morgan, B. J.; Zeier, W. G. Inducing High Ionic Conductivity in the Lithium Superionic Argyrodites $\mathrm{Li}_{6+x} \mathrm{P}_{1-x} \mathrm{Ge}_{x} \mathrm{~S}_{5} \mathrm{I}$ for All-Solid-State Batteries. Journal of the American Chemical Society 2018, 140, 16330-16339.

(483) Deiseroth, H.-J.; Kong, S.-T.; Eckert, H.; Vannahme, J.; Reiner, C.; Zai $\beta$, T.; Schlosser, M. $\mathrm{Li}_{6} \mathrm{PS}_{5} X$ : A Class of Crystalline Li-Rich Solids With an Unusually High $\mathrm{Li}^{+}$Mobility. Angewandte Chemie International Edition 2008, 47, 755-758.

(484) Kraft, M. A.; Culver, S. P.; Calderon, M.; Böcher,F.; Krauskopf, T.; Senyshyn, A.; Dietrich, C.; Zevalkink, A.; Janek, J.; Zeier, W. G. Influence of lattice polarizability on the ionic conductivity in the lithium superionic argyrodites $\mathrm{Li}_{6} \mathrm{PS}_{5} X(X=\mathrm{Cl}, \mathrm{Br}$, I). Journal of the American Chemical Society 2017, 139, 10909-10918.

(485) Minafra, N.; Culver, S. P.; Krauskopf, T.; Senyshyn, A.; Zeier, W. G. Effect of Si substitution on the structural and transport properties of superionic Li-argyrodites. Journal of Materials Chemistry A 2018, 6, 645-651.

(486) Adeli, P.; Bazak, J. D.; Park, K. H.; Kochetkov, I.; Huq, A.; Goward, G. R.; Nazar, L. F. Boosting solid-state diffusivity and conductivity in lithium superionic argyrodites by halide substitution. Angewandte Chemie International Edition 2019, $58,8681-8686$.

(487) Kuhn, A.; Duppel, V.; Lotsch, B. V. Tetragonal $\mathrm{Li}_{10} \mathrm{GeP}_{2} \mathrm{~S}_{12}$ - exploring the Li ion dynamics in LGPS Li electrolytes. Energy Environ. Sci. 2013, 6, 3548-3552.

(488) Xu, M.; Ding, J.; Ma, E. One-dimensional stringlike cooperative migration of lithium ions in an ultrafast ionic conductor. Applied Physics Letters 2012, 101, 031901.

(489) Adams, S.; Prasada Rao, R. Structural requirements for fast lithium ion migration in $\mathrm{Li}_{10} \mathrm{GeP}_{2} \mathrm{~S}_{12}$. Journal of Materials Chemistry 2012, 22, 7687. 
(490) Kuhn, A.; Köhler, J.; Lotsch, B. V. Single-crystal X-ray structure analysis of the superionic conductor $\mathrm{Li}_{10} \mathrm{GeP}_{2} \mathrm{~S}_{12}$. Phys. Chem. Chem. Phys. 2013, 15, 11620-2.

(491) Zhou, L.; Minafra, N.; Zeier, W. G.; Nazar, L. F. Innovative Approaches to LiArgyrodite Solid Electrolytes for All-Solid-State Lithium Batteries. Accounts of Chemical Research 2021, 19002-19013.

(492) Bachman, J. C.; Muy, S.; Grimaud, A.; Chang, H.-H.; Pour, N.; Lux, S. F.; Paschos, O.; Maglia, F.; Lupart, S.; Lamp, P., et al. Inorganic solid-state electrolytes for lithium batteries: mechanisms and properties governing ion conduction. Chemical reviews 2016, 116, 140-162.

(493) Ohno, S.; Helm, B.; Fuchs, T.; Dewald, G.; Kraft, M./A.; Culver, S. P.; Senyshyn, A.; Zeier, W. G. Further Evidence for Energy Landscape Flattening in the Superionic Argyrodites $\mathrm{Li}_{6+x} \mathrm{P}_{1-x} \mathrm{M}_{x} \mathrm{~S}_{5} \mathrm{I}(\mathrm{M}=\mathrm{Si}, \mathrm{Ge}, \mathrm{Sn})$. Chemistry of Materials 2019, 31, 49364944.

(494) Zhao, F.; Liang, J.; Yu, C.; Sun, Q.; Li, X.; Adair, K.; Wang, C.; Zhao, Y.; Zhang, S.; Li, W., et al. A Versatile Sn-Substituted Argyrodite Sulfide Electrolyte for All-SolidState Li Metal Batteries. Adv. Energy Mater. 2020, 10, 1903422.

(495) Zhou, L.; Assoud, A.; Zhang, Q.; Wu, X.; Nazar, L. F. New Family of Argyrodite Thioantimonate Lithium Superionic Conductors. J. Am. Chem. Soc. 2019, 141, 19002-19013.

(496) Zhou, L.; Park, K.-H.; Sun, X.; Lalère, F.; Adermann, T.; Hartmann, P.; Nazar, L. F. Solvent-Engineered Design of Argyrodite $\mathrm{Li}_{6} \mathrm{PS}_{5} X(X=\mathrm{Cl}, \mathrm{Br}, \mathrm{I})$ Solid Electrolytes with High Ionic Conductivity. ACS Energy Letters 2019, 4, 265-270.

(497) Wang, P.; Liu, H.; Patel, S.; Feng, X.; Chien, P.-H.; Wang, Y.; Hu, Y.-Y. Fast Ion Conduction and Its Origin in $\mathrm{Li}_{6-x} \mathrm{PS}_{5-x} \mathrm{Br}_{1+x}$. Chem. Mater. 2020, 32, 3833-3840. 
(506) Murugan, R.; Thangadurai, V.; Weppner, W. Fast lithium ion conduction in garnet$\mathrm{Li}_{6} \mathrm{PS}_{5} X$ Argyrodites. Chem. Mater. 2021, 33, 2004-2018.

(499) Kuhs, W.; Nitsche, R.; Scheunemann, K. The argyrodites- A new family of tetrahedrally close-packed structures. Materials Research Bulletin 1979,14, 241-248.

(500) Gautam, A.; Sadowski, M.; Ghidiu, M.; Minafra, N.; Senyshyn, A.; Albe, K.; Zeier, W. G. Engineering the Site-Disorder and Lithium Distribution in the Lithium Superionic Argyrodite $\mathrm{Li}_{6} \mathrm{PS}_{5} \mathrm{Br}$. Advanced Energy Materials 11, 2003369.

(501) Minafra, N.; Kraft, M.; Bernges, T.; Li, C.; Schlem, R.; Morgan, B.; Zeier, W. On the Local Charge Inhomogeneity and Lithium Distribution in the Superionic Argyrodites Li6PS5X $(\mathrm{X}=\mathrm{Cl}, \mathrm{Br}, \mathrm{I}) .2020$,

(502) Deng, Z.; Zhu, Z.; Chu, I.-H.; Ong, S. P. Data-Driven First-Principles Methods for the Study and Design of Alkali Superionic Conductors. Chemistry of Materials 2017, 29, 281-288.

(503) Yu, C. et al. Superionic conductivity in lithium argyrodite solid-state electrolyte by controlled Cl-doping. Nano Energy 2020, 69, 104396.

(504) Feng, X.; Chien, P.-H.; Wang, Y.; Patel, S.; Wang, P.; Liu, H.; Immediato-Scuotto, M.; $\mathrm{Hu}, \mathrm{Y} .-\mathrm{Y}$. Enhanced ion conduction by enforcing structural disorder in Li-deficient argyrodites $\mathrm{Li}_{6-x} \mathrm{PS}_{5-x} \mathrm{Cl}_{1+x}$. Energy Storage Materials 2020, 30, $67-73$.

(505) Yu, C.; Hageman, J.; Ganapathy, S.; van Eijck, L.; Zhang, L.; Adair, K. R.; Sun, X.; Wagemaker, M. Tailoring $\mathrm{Li}_{6} \mathrm{PS}_{5} \mathrm{Br}$ ionic conductivity and understanding of its role in cathode mixtures for high performance all-solid-state Li-S batteries. Journal of Materials Chemistry A 2019, 7, 10412-10421. type $\mathrm{Li}_{7} \mathrm{La}_{3} \mathrm{Zr}_{2} \mathrm{O}_{1}$ 2. Angewandte Chemie - International Edition 2007, 46, 7778-7781.

(498) Morgan, B. J. Mechanistic Origin of Superionic Lithium Diffusion in Anion-Disordered 
${ }_{4443}$ (507) Ni, J. E.; Case, E. D.; Sakamoto, J. S.; Rangasamy, E.; Wolfenstine, J. B. Room

4444

4445

4446

4447

4448

4449

4450

4451

4452

4453

4454

4455

4456

4457

4458

4459

4460

4461

4462

4463

4464

4465

4466

4467 temperature elastic moduli and Vickers hardness of hot-pressed LLZO cubic garnet. Journal of Materials Science 2012, 47, 7978-7985.

(508) Zhu, Y.; He, X.; Mo, Y. First principles study on electrochemical and chemical stability of solid electrolyte-electrode interfaces in all-solid-state Li-ion batteries. Journal of Materials Chemistry A 2016, 4, 3253-3266.

(509) Binninger, T.; Marcolongo, A.; Mottet, M.; Weber, V.; Laino, T. Comparison of computational methods for the electrochemical stability window of solid-state electrolyte materials. Journal of Materials Chemistry A 2020, 8, 1347-1359.

(510) Geiger, C. A.; Alekseev, E.; Lazic, B.; Fisch, M.; Armbruster, T.; Langner, R.; Fechtelkord, M.; Kim, N.; Pettke, T.; Weppner, W. Crystal chemistry and stability of " $\mathrm{Li}_{7} \mathrm{La}_{3} \mathrm{Zr}_{2} \mathrm{O}_{12}$ " garnet: A fast lithium-ion conductor. Inorganic Chemistry 2011, 50, 1089-1097.

(511) Rangasamy, E.; Wolfenstine, J.; Sakamoto, J. The role of Al and Li concentration on the formation of cubic garnet solid electrolyte of nominal composition $\mathrm{Li}_{7} \mathrm{La}_{3} \mathrm{Zr}_{2} \mathrm{O}_{12}$. Solid State Ionics 2012, 206, 28-32.

(512) Ren, Y.; Shen, Y.; Lin, Y.; Nan, C. W. Direct observation of lithium dendrites inside garnet-type lithium-ion solid electrolyte. Electrochemistry Communications 2015, 57, 27-30.

(513) Sudo, R.; Nakata, Y.; Ishiguro, K.; Matsui, M.; Hirano, A.; Takeda, Y.; Yamamoto, O.; Imanishi, N. Interface behavior between garnet-type lithium-conducting solid electrolyte and lithium metal. Solid State Ionics 2014, 262, 151-154.

(514) Cheng, E. J.; Sharafi, A.; Sakamoto, J. Intergranular Li metal propagation through polycrystalline $\mathrm{Li}_{6.25} \mathrm{Al}_{0.25} \mathrm{La}_{3} \mathrm{Zr}_{2} \mathrm{O}_{12}$ ceramic electrolyte. Electrochimica Acta $\mathbf{2 0 1 7}$, 
(515) Kim, S.; Jung, C.; Kim, H.; Thomas-Alyea, K. E.; Yoon, G.; Kim, B.; Badding, M. E.; Song, Z.; Chang, J. M.; Kim, J.; Im, D.; Kang, K. The Role of Interlayer Chemistry in Li-Metal Growth through a Garnet-Type Solid Electrolyte. Advanced Energy Materials 2020, 10, 1903993.

(516) Canepa, P.; Dawson, J. A.; Sai Gautam, G.; Statham, J. M.; Parker, S. C.; Islam, M. S. Particle Morphology and Lithium Segregation to Surfaces of the $\mathrm{Li}_{7} \mathrm{La}_{3} \mathrm{Zr}_{2} \mathrm{O}_{12}$ Solid Electrolyte. Chemistry of Materials 2018, 30, 3019-3027.

(517) Tian, H. K.; Xu, B.; Qi, Y. Computational study of lithium nucleation tendency in $\mathrm{Li}_{7} \mathrm{La}_{3} \mathrm{Zr}_{2} \mathrm{O}_{12}$ (LLZO) and rational design of interlayer materials to prevent lithium dendrites. Journal of Power Sources 2018, 392, 79-86.

(518) Gao, B.; Jalem, R.; Tateyama, Y. Surface-Dependent Stability of the Interface between Garnet $\mathrm{Li}_{7} \mathrm{La}_{3} \mathrm{Zr}_{2} \mathrm{O}_{12}$ and the Li Metal in the All-Solid-State Battery from FirstPrinciples Calculations. ACS Applied Materials \& Interfaces 2020, 12, 16350-16358.

(519) Ma, C.; Cheng, Y.; Yin, K.; Luo, J.; Sharafi, A.; Sakamoto, J.; Li, J.; More, K. L.; Dudney, N. J.; Chi, M. Interfacial Stability of Li Metal-Solid Electrolyte Elucidated via in Situ Electron Microscopy. Nano Letters 2016, 16, 7030-7036.

(520) Rettenwander, D.; Wagner, R.; Reyer, A.; Bonta, M.; Cheng, L.; Doeff, M. M.; Limbeck, A.; Wilkening, M.; Amthauer, G. Interface instability of fe-stabilized $\mathrm{Li}_{7} \mathrm{La}_{3} \mathrm{Zr}_{2} \mathrm{O}_{12}$ versus li metal. Journal of Physical Chemistry C 2018, 122, 3780-3785.

(521) Shen, F.; Dixit, M. B.; Xiao, X.; Hatzell, K. B. Effect of Pore Connectivity on Li Dendrite Propagation within LLZO Electrolytes Observed with Synchrotron X-ray Tomography. ACS Energy Letters 2018, 3, 1056-1061.

(522) Aguesse, F.; Manalastas, W.; Buannic, L.; Del Amo, J. M. L.; Singh, G.; Llordés, A.; Kilner, J. Investigating the dendritic growth during full cell cycling of garnet elec- 

trolyte in direct contact with Li metal. ACS Applied Materials and Interfaces 2017, 9, 3808-3816.

(523) Krauskopf, T.; Hartmann, H.; Zeier, W. G.; Janek, J. Toward a Fundamental Understanding of the Lithium Metal Anode in Solid-State Batteries - An ElectrochemoMechanical Study on the Garnet-Type Solid Electrolyte $\mathrm{Li}_{6.25} \mathrm{Al}_{0.25} \mathrm{La}_{3} \mathrm{Zr}_{2} \mathrm{O}_{12}$. ACS Applied Materials and Interfaces 2019, 11, 14463-14477.

(524) Zhu, Y.; Connell, J. G.; Tepavcevic, S.; Zapol, P.; Garcia-Mendez, R.; Taylor, N. J.; Sakamoto, J.; Ingram, B. J.; Curtiss, L. A.; Freeland, J. W.; Fong, D. D.; Markovic, N. M. Dopant-Dependent Stability of Garnet Solid Electrolyte Interfaces with Lithium Metal. Advanced Energy Materials 2019, 9, 1-11.

(525) Tsai, C.-L.; Roddatis, V.; Chandran, C. V.; Ma, Q.; Uhlenbruck, S.; Bram, M.; Heitjans, P.; Guillon, O. $\mathrm{Li}_{7} \mathrm{La}_{3} \mathrm{Zr}_{2} \mathrm{O}_{12}$ Interface Modification for Li Dendrite Prevention. ACS Applied Materials \& Interfaces 2016, 8, 10617-10626.

(526) Thompson, T.; Yu, S.; Williams, L.; Schmidt, R. D.; Garcia-Mendez, R.; Wolfenstine, J.; Allen, J. L.; Kioupakis, E.; Siegel, D. J.; Sakamoto, J. Electrochemical Window of the Li-Ion Solid Electrolyte $\mathrm{Li}_{7} \mathrm{La}_{3} \mathrm{Zr}_{2} \mathrm{O}_{12}$. ACS Energy Letters 2017, 2, 462-468.

(527) Yu, S.; Schmidt, R. D.; Garcia-Mendez, R.; Herbert, E.; Dudney, N. J.; Wolfenstine, J. B., Sakamoto, J.; Siegel, D. J. Elastic Properties of the Solid Electrolyte $\mathrm{Li}_{7} \mathrm{La}_{3} \mathrm{Zr}_{2} \mathrm{O}_{12}$ (LLZO), Chemistry of Materials 2016, 28, 197-206.

(528) Wang, Y.; Lv, J.; Zhu, L.; Ma, Y. CALYPSO: A method for crystal structure prediction. Computer Physics Communications 2012, 183, 2063-2070.

(529) Gao, B.; Gao, P.; Lu, S.; Lv, J.; Wang, Y.; Ma, Y. Interface structure prediction via CALYPSO method. Science Bulletin 2019, 64, 301-309. 
4516 (530) Han, F.; Westover, A. S.; Yue, J.; Fan, X.; Wang, F.; Chi, M.; Leonard, D. N.;

4517

4518

4519

4520

4521

4522

4523

4524

4525

4526

4527

4528

4529

4530

4531

4532

4533

4534

4535

4536

4537

4538 2012, 85, 052301. Review Letters 2016, 116, 1-6.

Dudney, N. J.; Wang, H.; Wang, C. High electronic conductivity as the origin of lithium dendrite formation within solid electrolytes. Nature Energy 2019, 4, 187-196.

(531) Squires, A.; Davies, D.; Kim, S.; Scanlon, D.; Walsh, A.; Morgan, B. Low Electronic Conductivity of $\mathrm{Li}_{7} \mathrm{La}_{3} \mathrm{Zr}_{2} \mathrm{O}_{12}$ (LLZO) Solid Electrolytes from/First Principles. 2020,

(532) Xu, M.; Park, M. S.; Lee, J. M.; Kim, T. Y.; Park, Y. S.; Ma, E. Mechanisms of $\mathrm{Li}^{+}$ transport in garnet-type cubic $\mathrm{Li}_{3+x} \mathrm{La}_{3} M_{2} \mathrm{O}_{12}(M=\mathrm{Te}, \mathrm{Nb}, \mathrm{Zr})$. Physical Review $B$

(533) Burbano, M.; Carlier, D.; Boucher, F.; Morgan, B. J.; Salanne, M. Sparse Cyclic Excitations Explain the Low Ionic Conductivity of Stoichometric $\mathrm{Li}_{7} \mathrm{La}_{3} \mathrm{Zr}_{2} \mathrm{O}_{12}$. Physical

(534) Meier, K.; Laino, T.; Curioni, A. Solid-state electrolytes: Revealing the mechanisms of Li-Ion conduction in tetragonal and cubic LLZO by first-principles calculations. Journal of Physical Chemistry C 2014, 118, 6668-6679.

(535) Jalem, R.; Yamamoto, Y.; Shiiba, H.; Nakayama, M.; Munakata, H.; Kasuga, T.; Kanamura, K. Concerted migration mechanism in the Li ion dynamics of garnet-type $\mathrm{Li}_{7} \mathrm{La}_{3} \mathrm{Zr}_{2} \mathrm{O}_{1} 2$. Chemistry of Materials 2013, 25, 425-430.

(536) Rettenwander, D.; Blaha, P.; Laskowski, R.; Schwarz, K.; Bottke, P.; Wilkening, M.; Geiger, C.A.; Amthauer, G. DFT study of the role of $\mathrm{Al}^{3+}$ in the fast ion-conductor $\mathrm{Li}_{7-3 x} \mathrm{Al}_{34 x} \mathrm{La}_{3} \mathrm{Zr}_{2} \mathrm{O}_{12}$ garnet. Chemistry of Materials 2617-2623.

(537) Rettenwander, D.; Redhammer, G.; Preishuber-Pflügl, F.; Cheng, L.; Miara, L.; Wagner, R.; Welzl, A.; Suard, E.; Doeff, M. M.; Wilkening, M.; Fleig, J.; Amthauer, G. Structural and Electrochemical Consequences of $\mathrm{Al}$ and Ga Cosubstitution in $\mathrm{Li}_{7} \mathrm{La}_{3} \mathrm{Zr}_{2} \mathrm{O}_{12}$ Solid Electrolytes. Chemistry of Materials 2016, 28, 2384-2392. 
4540

4541

4542

4543

4544

4545

4546

4547

4548

4549

4550

4551

4552

4553

4554

4555

4556

4557

4558

4559

4560

4561

4562

(538) Bonilla, M. R.; García Daza, F. A.; Carrasco, J.; Akhmatskaya, E. Exploring Li-ion conductivity in cubic, tetragonal and mixed-phase Al-substituted $\mathrm{Li}_{7} \mathrm{La}_{3} \mathrm{Zr}_{2} \mathrm{O}_{12}$ using atomistic simulations and effective medium theory. Acta Materialia 2019, 175, 426435.

(539) Awaka, J.; Kijima, N.; Hayakawa, H.; Akimoto, J. Synthesis and structure analysis of tetragonal $\mathrm{Li}_{7} \mathrm{La}_{3} \mathrm{Zr}_{2} \mathrm{O}_{12}$ with the garnet-related type structure. Journal of Solid State Chemistry 2009, 182, 2046-2052.

(540) Hayamizu, K.; Seki, S.; Haishi, T. Lithium ion micrometer diffusion in a garnet-type cubic $\mathrm{Li}_{7} \mathrm{La}_{3} \mathrm{Zr}_{2} \mathrm{O}_{12}$ (LLZO) studied using ${ }^{7} \mathrm{Li} \mathrm{NMR}$ spectroscopy. The Journal of Chemical Physics 2017, 146, 024701.

(541) Brugge, R. H.; Chater, R. J.; Kilner, J. A.; Aguadero, A. Experimental determination of Li diffusivity in LLZO using isotopic exchange and FIB-SIMS. Journal of Physics: Energy 2021, 3, 034001.

(542) Sharafi, A.; Yu, S.; Naguib, M.; Lee, M.; Ma, C.; Meyer, H. M.; Nanda, J.; Chi, M.; Siegel, D. J.; Sakamoto, J. Impact of air exposure and surface chemistry on Li$\mathrm{Li}_{7} \mathrm{La}_{3} \mathrm{Zr}_{2} \mathrm{O}_{12}$ interfacial resistance. Journal of Materials Chemistry A 2017, 5, 1347513487 .

(543) Islam, M. M.; Bredow, T.; Heitjans, P. The ionic conductivity in lithium-boron oxide materials and its relation to structural, electronic and defect properties: insights from theory. Journal of Physics: Condensed Matter 2012, 24, 203201.

(544) Islam, M. M.; Bredow, T.; Indris, S.; Heitjans, P. Enhanced Conductivity at the Interface of $\mathrm{Li}_{2} \mathrm{O}: \mathrm{B}_{2} \mathrm{O}_{3}$ Nanocomposites: Atomistic Models. Phys. Rev. Lett. 2007, 99, 145502.

4563 (545) Baggio, B. F.; Grunder, Y. In Situ X-Ray Techniques for Electrochemical Interfaces. 

Annual Review of Analytical Chemistry 2021, 14, DOI: 10.1146/annurev-anchem091020-100631.

(546) Claessen, R.; Sing, M.; Paul, M.; Berner, G.; Wetscherek, A.; Müller, A.; Drube, W. Hard x-ray photoelectron spectroscopy of oxide hybrid and heterostructures: a new method for the study of buried interfaces. New Journal of Physies 2009, 11, 125007.

(547) Thangadurai, V.; Weppner, W. $\mathrm{Li}_{6} \mathrm{ALa}_{2} \mathrm{Ta}_{2} \mathrm{O}_{12}(\mathrm{~A}=\mathrm{Sr}, \mathrm{Ba})$ : Novel garnet-like oxides for fast lithium ion conduction. Advanced Functional Materials 2005, 15, 107-112.

(548) Liu, Z.; Fu, W.; Payzant, E. A.; Yu, X.; Wu, Z.; Dudney, N. J.; Kiggans, J.; Hong, K.; Rondinone, A. J.; Liang, C. Anomalous High Ionic Conductivity of Nanoporous $\beta$-Li 3 PS 4. Journal of the American Chemical Society 2013, 135, 975-978.

(549) Kobayashi, T.; Yamada, A.; Kanno, R. Interfacial reactions at electrode/electrolyte boundary in all solid-state lithium battery using inorganic solid electrolyte, thioLISICON. Electrochimica Acta 2008, 53, 5045-5050.

(550) Takada, K.; Ohta, N.; Zhang, L.; Fukuda, K.; Sakaguchi, I.; Ma, R.; Osada, M.; Sasaki, T. Interfacial modification for high-power solid-state lithium batteries. Solid State Ionics 1333-1337.

(551) Sakuda, A.; Hayashi, A.; Tatsumisago, M. Interfacial observation between $\mathrm{LiCoO}_{2}$ electrode and $\mathrm{Li}_{2} \mathrm{~S}-\mathrm{P}_{2} \mathrm{~S}_{5}$ solid electrolytes of all-solid-state lithium secondary batteries using transmission electron microscopy. Chemistry of Materials 2010, 22, 949-956.

(552) Okuno, Y.; Haruyama, J.; Tateyama, Y. Comparative study on sulfide and oxide electrolyte interfaces with cathodes in all-solid-state battery via first-principles calculations. ACS Applied Energy Materials 2020, 3, 11061-11072.

(553) Sharafi, A.; Kazyak, E.; Davis, A. L.; Yu, S.; Thompson, T.; Siegel, D. J.; Dasgupta, N. P.; Sakamoto, J. Surface Chemistry Mechanism of Ultra-Low Interfacial 

Resistance in the Solid-State Electrolyte $\mathrm{Li}_{7} \mathrm{La}_{3} \mathrm{Zr}_{2} \mathrm{O}_{12}$. Chemistry of Materials $\mathbf{2 0 1 7}$, 29, 7961-7968.

(554) Jiang, Z.; Han, Q.; Wang, S.; Wang, H. Reducing the Interfacial Resistance in All-Solid-State Lithium Batteries Based on Oxide Ceramic Electrolytes. ChemElectroChem 2019, 6, 2970-2983.

(555) Lepley, N. D.; Holzwarth, N. A. Modeling interfaces between solids: Application to Li battery materials. Physical Review B - Condensed Matter and Materials Physics 2015, 92, 1-15.

(556) Zhang, Y. Q.; Tian, Y.; Xiao, Y.; Miara, L. J.; Aihara, Y.; Tsujimura, T.; Shi, T.; Scott, M. C.; Ceder, G. Direct Visualization of the Interfacial Degradation of Cathode Coatings in Solid State Batteries: A Combined Experimental and Computational Study. Advanced Energy Materials 2020, 10.

(557) Xiao, Y.; Miara, L. J.; Wang, Y.; Ceder, G. Computational Screening of Cathode Coatings for Solid-State Batteries. Joule 2019, 3, 1252-1275.

(558) Sang, J.; Yu, Y.; Wang, Z.; Shao, G. Theoretical formulation of $\operatorname{Li}_{3 a+b} \mathrm{~N}_{a} \mathrm{X}_{b}$ (X = halogen) as a potential artificial solid electrolyte interphase (ASEI) to protect the $\mathrm{Li}$ anode. Physical Chemistry Chemical Physics 2020, 22, 12918-12928.

(559) Glass, C. W . Oganov, A. R.; Hansen, N. USPEX-Evolutionary crystal structure prediction. Computer Physics Communications 2006, 175, 713-720.

(560) Oganov, A. R.; Glass, C. W. Crystal structure prediction using ab initio evolutionary techniques: Principles and applications. Journal of Chemical Physics 2006, 124.

(561) Parlinski, K.; Li, Z. Q.; Kawazoe, Y. First-principles determination of the soft mode in cubic $\mathrm{ZrO}_{2}$. Physical Review Letters 1997, 78, 4063-4066. 
4611 (562) Togo, A.; Oba, F.; Tanaka, I. First-principles calculations of the ferroelastic transition between rutile-type and $\mathrm{CaCl}_{2}$-type $\mathrm{SiO}_{2}$ at high pressures. Physical Review B Condensed Matter and Materials Physics 2008, 78, 1-9.

4614

4615

4616

4617

4618

4619

4620

4621

4622

4623

4624

4625

4626

4627

4628

4629

4630

4631

4632

4633

(563) Hart, G. L.; Forcade, R. W. Algorithm for generating derivative structures. Physical Review B - Condensed Matter and Materials Physics 2008, 7\%, 1-12.

(564) Sang, L.; Kissoon, N.; Wen, F. Characterizations of dynamic interfaces in all-solid lithium batteries. Journal of Power Sources 2021, 506.

(565) Schnell, J.; Knörzer, H.; Imbsweiler, A. J.; Reinhart, G. Solid versus Liquid-A Bottom-Up Calculation Model to Analyze the Manufacturing Cost of Future HighEnergy Batteries. Energy Technology 2020, 8, 1901237.

(566) Kato, Y.; Hori, S.; Saito, T.; Suzuki, K.; Hirayama, M.; Mitsui, A.; Yonemura, M.; Iba, H.; Kanno, R. High-power all-solid-state batteries using sulfide superionic conductors. Nature Energy 2016, 1, 1-7.

(567) Kim, K. J.; Balaish, M.; Wadaguchi, M.; Kong, L.; Rupp, J. L. Solid-State Li-Metal Batteries: Challenges and Horizons of Oxide and Sulfide Solid Electrolytes and Their Interfaces. Advanced Energy Materials 2021, 11, 2002689.

(568) Albertus, P.; Babinec, S.; Litzelman, S.; Newman, A. Status and challenges in enabling the lithium metal electrode for high-energy and low-cost rechargeable batteries. Nature Energy 2018, 3, 16-21.

(569) Rodney, D.; Ventelon, L.; Clouet, E.; Pizzagalli, L.; Willaime, F. Ab initio modeling of dislocation core properties in metals and semiconductors. Acta Materialia 2017, $124,633-659$.

(570) Clouet, E. In Handbook of Materials Modeling: Methods: Theory and Modeling; Andreoni, W., Yip, S., Eds.; Springer International Publishing, 2020; pp 1503-1524. 

istry 2021, 52, 202-209.

(571) Chen, H.; Liu, Q. Y.; Jing, M. X.; Chen, F.; Yuan, W. Y.; Ju, B. W.; Tu, F. Y.; Shen, X. Q.; Qin, S. B. Improved Interface Stability and Room-Temperature Performance of Solid-State Lithium Batteries by Integrating Cathode/Electrolyte and Graphite Coating. ACS Applied Materials and Interfaces 2020, 12,15120-15127,

(572) Ito, Y.; Yamakawa, S.; Hayashi, A.; Tatsumisago, M. Effects of the microstructure of solid-electrolyte-coated $\mathrm{LiCoO}_{2}$ on its discharge properties in all-solid-state lithium batteries. Journal of Materials Chemistry A 2017, 5, 10658-10668.

(573) Yin, Y.; Jiang, C.-S.; Guthrey, H.; Xiao, C.; Seitzman, N.; Ban, C.; Al-Jassim, M. Improved Stability and Cyclability of Ceramic Solid Electrolyte by Coating Polymer. Journal of The Electrochemical Society 2020, 167, 020519.

(574) Ji, X.; Hou, S.; Wang, P.; He, X.; Piao, N.; Chen, J.; Fan, X.; Wang, C. Solid-State Electrolyte Design for Lithium Dendrite Suppression. Advanced Materials 2020, 32.

(575) Li, H.; Lian, F.; Meng, N.; Xiong, C.; Wu, N.; Xu, B.; Li, Y. Constructing Electronic and Ionic Dual Conductive Polymeric Interface in the Cathode for High-EnergyDensity Solid-State Batteries. Advanced Functional Materials 2021, 31, 2008487.

(576) Yi, J.; He, P.; Liu, H.; Ni, H.; Bai, Z.; Fan, L. Z. Manipulating interfacial stability of $\mathrm{LiNi}_{0.5} \mathrm{Co}_{0.3} \mathrm{Mn}_{0.2} \mathrm{O}_{2}$ cathode with sulfide electrolyte by nanosized LLTO coating to achieve high-performance all-solid-state lithium batteries. Journal of Energy Chem-

(577) Dai, H.; Chen, Y.; Xú, W.; Hu, Z.; Gu, J.; Wei, X.; Xie, F.; Zhang, W.; Wei, W.; Guo,R.; Zhang, G. A Review of Modification Methods of Solid Electrolytes for AllSolid-State Sodium-Ion Batteries. Energy Technology 2021, 9, 1-13.

(578) High Voltage Stable Polyoxalate Catholyte with Cathode Coating for All-Solid-State Li-Metal/NMC622 Batteries. Advanced Energy Materials 2020, 10, 1-11. 
(579) Jing, M. X.; Yuan, W. Y.; Shen, X. Q.; Zhang, L. K.; Yang, H.; Liu, Q. Y.; Chen, F.; Liu, M. Q.; Ji, Y. S. Highly efficient interface modification between poly(propylene carbonate)-based solid electrolytes and a lithium anode by facile graphite coating. ACS Sustainable Chemistry and Engineering 2020, 8, 17106-17115.

(580) Wang, C.; Sun, X.; Yang, L.; Song, D.; Wu, Y.; Ohsaka, T.; Matsumoto, F.; Wu, J. In Situ Ion-Conducting Protective Layer Strategy to Stable Lithium Metal Anode for All-Solid-State Sulfide-Based Lithium Metal Batteries. Advanced Materials Interfaces $2021,8,1-10$.

(581) Zhao, F. et al. Tuning bifunctional interface for advanced sulfide-based all-solid-state batteries. Energy Storage Materials 2020, 33,139-146.

(582) Zhao, B.; Feng, X.; Yu, M.; Wang, W.; Hao, S.; Chen, H.; Huang, Y.; Gong, W.; Liu, L.; Qiu, H. Interfacial modification enabled room temperature solid-state lithiummetal batteries. Ionics 2021, 27, 1569-1578.

(583) Liang, J. et al. Stabilizing and understanding the interface between nickel-rich cathode and PEO-based electrolyte by lithium niobium oxide coating for high-performance allsolid-state batteries. Nano Energy 2020, 78, 105107.

(584) Zhang, Q.; Bruck, A. M.; Stavola, A. M.; Liang, W.; Aurora, P.; Gallaway, J. W. Enhanced Electrochemical Stability of Sulfide-Based $\mathrm{LiNi}_{0.8} \mathrm{Mn}_{0.1} \mathrm{Co}_{0.1} \mathrm{O}_{2}$ All-SolidState Batteries by Ti Surface Doping. Batteries $\&$ Supercaps 2020, 1-8.

(585) Mizushima, K.; Jones, P.; Wiseman, P.; Goodenough, J. B. $\mathrm{Li}_{x} \mathrm{CoO}_{2}(0<x<-1)$ : A new cathode material for batteries of high energy density. Materials Research Bulletin $\mathbf{1 9 8 0}, 15,783-789$.

(586) Rozier, P.; Tarascon, J. M. Li-rich layered oxide cathodes for next-generation Li-ion batteries: chances and challenges. Journal of The Electrochemical Society 2015, 162, 
(587) Whittingham, M. S. Materials challenges facing electrical energy storage. MRS Bulletin 2008, 33, 411-419.

(588) Dunn, B.; Kamath, H.; Tarascon, J.-M. Electrical energy storage for the grid: a battery of choices. Science 2011, 334, 928-935.

(589) Liu, J.; Zhang, J.-G.; Yang, Z.; Lemmon, J. P.; Imhoff, O.; Graff, G. L.; Li, L.; Hu, J.; Wang, C.; Xiao, J., et al. Materials science and materials chemistry for large scale electrochemical energy storage: from transportation to electrical grid. Advanced Functional Materials 2013, 23, 929-946.

(590) Palacin, M. R. Recent advances in rechargeable battery materials: a chemist's perspective. Chemical Society Reviews 2009, 38, 2565-2575.

(591) Masquelier, C.; Croguennec, L. Polyanionic (phosphates, silicates, sulfates) frameworks as electrode materials for rechargeable $\mathrm{Li}$ (or Na) batteries. Chemical Reviews 2013, 113, 6552-6591.

(592) Bruce, P. G.; Freunberger, S. A.; Hardwick, L. J.; Tarascon, J.-M. Li--O 2 and Li-S batteries with high energy storage. Nature materials 2012, 11, 19.

(593) Scrosati, B.; Hassoun, J.; Sun, Y.-K. Lithium-ion batteries. A look into the future. Energy \& Environmental Science 2011, 4, 3287-3295.

(594) Etacheri, V.; Marom, R.; Elazari, R.; Salitra, G.; Aurbach, D. Challenges in the development of advanced Li-ion batteries: a review. Energy \& Environmental Science 2011, 4, 3243-3262,

(595) Takada, K. Progress and prospective of solid-state lithium batteries. Acta Materialia 2013, 61, 759-770.

(596) Fergus, J. W. Recent developments in cathode materials for lithium ion batteries. Journal of power sources 2010, 195, 939-954. 
4713

4714

4715

4716

4717

4718

4719

4720

4721

4722

4723

4724

4725

4726

4727

4728

4729

4730

(597) Ellis, B. L.; Lee, K. T.; Nazar, L. F. Positive electrode materials for Li-ion and Libatteries. Chemistry of materials 2010, 22, 691-714.

(598) He, P.; Yu, H.; Zhou, H., et al. Layered lithium transition metal oxide cathodes towards high energy lithium-ion batteries. Journal of Materials Chemistry 2012, 22, 36803695 .

(599) Zaghib, K.; Guerfi, A.; Hovington, P.; Vijh, A.; Trudeau, M.; Mauger, A.; Goodenough, J.; Julien, C. Review and analysis of nanostructured olivine-based lithium recheargeable batteries: Status and trends. Journal of Power Sources 2013, 232, 357-369.

(600) Ellis, B. L.; Nazar, L. F. Sodium and sodium-ion energy storage batteries. Current Opinion in Solid State and Materials Science 2012, 16, 168-177.

(601) Kim, S.-W.; Seo, D.-H.; Ma, X.; Ceder, G.; Kang, K. Electrode materials for rechargeable sodium-ion batteries: potential alternatives to current lithium-ion batteries. $A d$ vanced Energy Materials 2012, 2, 710-721.

(602) Palomares, V.; Serras, P.; Villaluenga, I.; Hueso, K. B.; Carretero-González, J.; Rojo, T. Na-ion batteries, recent advances and present challenges to become low cost energy storage systems. Energy $\mathscr{E}$ Environmental Science 2012, 5, 5884-5901.

(603) Fergus, J. W. Ion transport in sodium ion conducting solid electrolytes. Solid State Ionics 2012, 22\%, 102-112.

(604) Yabuuchi, N.; Kajiyama, M.; Iwatate, J.; Nishikawa, H.; Hitomi, S.; Okuyama, R.; Usui, R., Yamada, Y.; Komaba, S. P2-type $\mathrm{Na}_{x}\left[\mathrm{Fe}_{1 / 2} \mathrm{Mn}_{1 / 2}\right] \mathrm{O}_{2}$ made from earthabundant elements for rechargeable Na batteries. Nature materials 2012, 11, 512-517.

(605) Daniel, C.; Mohanty, D.; Li, J.; Wood, D. L. Cathode materials review. 2014, 1597, $26-43$. 
${ }_{4732}$ (606) Islam, M. S.; Fisher, C. A. Lithium and sodium battery cathode materials: computa-

4733

4734

4735

4736

4737

4738

4739

4740

4741

4742

4743

4744

4745

4746

4747

4748

4749

4750

4751

4752

4753

4754

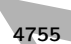
tional insights into voltage, diffusion and nanostructural properties. Chemical Society Reviews 2014, 43, 185-204.

(607) Myung, S.-T.; Maglia, F.; Park, K.-J.; Yoon, C. S.; Lamp, P.; Kim, S.-J.; Sun, Y.-K. Nickel-rich layered cathode materials for automotive lithium-ion batteries: achievements and perspectives. ACS Energy Letters 2017, 2, 196-223.

(608) Mo, J. Y.; Jeon, W. The impact of electric vehicle demand and battery recycling on price dynamics of lithium-ion battery cathode materials: A vector error correction model (VECM) analysis. Sustainability 2018, 10, 2870.

(609) Goonetilleke, D.; Sharma, N.; Pang, W. K.; Peterson, V. K.; Petibon, R.; Li, J.; Dahn, J. Structural Evolution and High-Voltage Structural Stability of $\mathrm{Li}\left(\mathrm{Ni}_{x} \mathrm{Mn}_{y} \mathrm{Co}_{z}\right) \mathrm{O}_{2}$ Electrodes. Chemistry of Materials 2018, 31, 376-386.

(610) Chen, Z.; Lu, Z.; Dahn, J. Staging phase transitions in $\mathrm{Li}_{x} \mathrm{CoO}_{2}$. Journal of the Electrochemical Society 2002, 149, A1604.

(611) Min, K.; Kim, K.; Jung, C.; Seo,S.-W.; Song, Y. Y.; Lee, H. S.; Shin, J.; Cho, E. A comparative study of structural changes in lithium nickel cobalt manganese oxide as a function of Ni content during delithiation process. Journal of Power Sources 2016, $315,111-119$.

(612) Tian, Z.; Yu, H.; Zhang, Z.; Xu, X. Performance Improvements of Cobalt Oxide Cathodes for Rechargeable Lithium Batteries. ChemBioEng Reviews 2018, 5, 111118.

(613) Paulsen, J.; Thomas, C.; Dahn, J. O ${ }_{2}$ Structure $\mathrm{Li}_{2 / 3}\left[\mathrm{Ni}_{1 / 3} \mathrm{Mn}_{2 / 3}\right] \mathrm{O}_{2}$ : A New Layered Cathode Material for Rechargeable Lithium Batteries. I. Electrochemical Properties. 
4756 (614) Paulsen, J.; Dahn, J. O ${ }_{2}$-Type $\mathrm{Li}_{2 / 3}\left[\mathrm{Ni}_{1 / 3} \mathrm{Mn}_{2 / 3}\right] \mathrm{O}_{2}$ : A New Layered Cathode Mate-

4757

4758

4759

4760

4761

4762

4763

4764

4765

4766

4767

4768

4769

4770

4771

4772

4773

4774

4775

4776

4777

4778 rial for Rechargeable Lithium Batteries II. Structure, Composition, and Properties. Journal of the Electrochemical Society 2000, 147, 2478.

(615) Lu, Z.; MacNeil, D.; Dahn, J. Layered Li[ $\left[\mathrm{Ni}_{x} \mathrm{Co}_{1-2 x} \mathrm{Mn}_{x}\right] \mathrm{O}_{2}$ Cathode materials for lithium-ion batteries. Electrochemical and Solid State Letters 2001, 4, A200.

(616) Ohzuku, T.; Makimura, Y. Layered lithium insertion material of $\mathrm{LiNi}_{1 / 2} \mathrm{Mn}_{1 / 2} \mathrm{O}_{2}$ : A possible alternative to $\mathrm{LiCoO}_{2}$ for advanced lithium-ion batteries. Chemistry Letters 2001, 30, 744-745.

(617) Duan, Y.; Yang, L.; Zhang, M.-J.; Chen, Z.; Bai, J.; Amine, K.; Pan, F.; Wang, F. Insights into $\mathrm{Li} / \mathrm{Ni}$ ordering and surface reconstruction during synthesis of Ni-rich layered oxides. Journal of Materials Chemistry A 2019, 7, 513-519.

(618) Zhang, N.; Li, J.; Li, H.; Liu, A.; Huang, Q.; Ma, L.; Li, Y.; Dahn, J. R. Structural, Electrochemical, and Thermal Properties of Nickel-Rich $\mathrm{LiNi}_{x} \mathrm{Mn}_{y} \mathrm{Co}_{z} \mathrm{O}_{2}$ Materials. Chemistry of Materials 2018, 30, 8852-8860.

(619) Azevedo, M.; Campagnol, N.; Hagenbruch, T.; Hoffman, K.; Lala, A.; Ramsbottom, O. Metals and Mining 2018, https://www.mckinsey.com/industries/metals-andmining/our-insights/lithium-and-cobalt-a-tale-of-two-commodities.

(620) Sathiya, M.; Rousse, G.; Ramesha, K.; Laisa, C.; Vezin, H.; Sougrati, M. T.; Doublet, M.-L; Foix, D.; Gonbeau, D.; Walker, W., et al. Reversible anionic redox chemistry in high-capacity/layered-oxide electrodes. Nature materials 2013, 12, 827-835.

(621) Lee, J.; Urban, A.; Li, X.; Su, D.; Hautier, G.; Ceder, G. Unlocking the potential of cation-disordered oxides for rechargeable lithium batteries. Science 2014, 343, 519522. 
4779 (622) Oishi, M.; Yogi, C.; Watanabe, I.; Ohta, T.; Orikasa, Y.; Uchimoto, Y.; Ogumi, Z.

4780

4781

4782

4783

4784

4785

4786

4787

4788

4789

4790

4791

4792

4793

4794

4795

4796

4797

4798

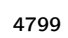

4800

4801

Direct observation of reversible charge compensation by oxygen ion in Li-rich manganese layered oxide positive electrode material, $\mathrm{Li}_{1.16} \mathrm{Ni}_{0.15} \mathrm{Co}_{0.19} \mathrm{Mn}_{0.50} \mathrm{O}_{2}$. Journal of Power Sources 2015, 276, 89-94.

(623) Seo, D.-H.; Lee, J.; Urban, A.; Malik, R.; Kang, S.; Ceder, G. The structural and chemical origin of the oxygen redox activity in layered and cation-disordered Li-excess cathode materials. Nature Chemistry 2016, 8, 692-697.

(624) Gent, W. E. et al. Coupling between oxygen redox and cation migration explains unusual electrochemistry in lithium-rich layered oxides. Nature Communications $\mathbf{2 0 1 7}$, 8,2091 .

(625) Assat, G.; Tarascon, J.-M. Fundamental understanding and practical challenges of anionic redox activity in Li-ion batteries. Nature Energy 2018, 3, 373-386.

(626) Naylor, A. J.; Makkos, E.; Maibach, J.; Guerrini, N.; Sobkowiak, A.; Björklund, E.; Lozano, J. G.; Menon, A. S.; Younesi, R.; Roberts, M. R., et al. Depth-dependent oxygen redox activity in lithium-rich layered oxide cathodes. Journal of Materials Chemistry A 2019, 7, 25355-25368.

(627) House, R. A.; Maitra, U.; Pérez-Osorio, M. A.; Lozano, J. G.; Jin, L.; Somerville, J. W.; Duda, L. C.; Nag, A.; Walters, A.; Zhou, K.-J.; Roberts, M. R.; Bruce, P. G. Superstructure control of first-cycle voltage hysteresis in oxygen-redox cathodes. Nature 2020, 577, 502-508.

(628) House, R. A.; Rees, G. J.; Pérez-Osorio, M. A.; Marie, J. J.; Boivin, E.; Robertson, A. W.; Nag, A.; Garcia-Fernandez, M.; Zhou, K. J.; Bruce, P. G. First-cycle voltage hysteresis in Li-rich $3 \mathrm{~d}$ cathodes associated with molecular $\mathrm{O}_{2}$ trapped in the bulk. Nature Energy 2020, 5, 777-785. 
${ }_{4803}$ (629) Hy, S.; Liu, H.; Zhang, M.; Qian, D.; Hwang, B.-J.; Meng, Y. S. Performance and

4804

4805

4806

4807

4808

4809

4810

4811

4812

4813

4814

4815

4816

4817

4818

4819

4820

4821

4822

4823

4824

4825 design considerations for lithium excess layered oxide positive electrode materials for lithium ion batteries. Energy \& Environmental Science 2016, 9, 1931-1954.

(630) Urban, A.; Lee, J.; Ceder, G. The Configurational Space of Rocksalt-Type Oxides for High-Capacity Lithium Battery Electrodes. Advanced Energy Materials 2014, 4, 1400478.

(631) Lee, J.; Seo, D.-H.; Balasubramanian, M.; Twu, N.; Li, X.; Ceder, G. A new class of high capacity cation-disordered oxides for rechargeable lithium batteries: Li-Ni-Ti-Mo oxides. Energy \& Environmental Science 2015, 8, 3255-3265.

(632) Yabuuchi, N.; Nakayama, M.; Takeuchi, M.; Komaba, S.; Hashimoto, Y.; Mukai, T.; Shiiba, H.; Sato, K.; Kobayashi, Y.; Nakao, A.; Yonemura, M.; Yamanaka, K.; Mitsuhara, K.; Ohta, T. Origin of stabilization and destabilization in solid-state redox reaction of oxide ions for lithium-ion batteries. Nature Communications 2016, 7, 110.

(633) Freire, M.; Kosova, N. V.; Jordy, C.; Chateigner, D.; Lebedev, O. I.; Maignan, A.; Pralong, V. A new active Li-Mn-O compound for high energy density Li-ion batteries. Nature Materials 2016, 15, 173-177.

(634) Yao, Z.; Kim, S.; He, J.;Hegde, V. I.; Wolverton, C. Interplay of cation and anion redox in $\mathrm{Li}_{4} \mathrm{Mn}_{2} \mathrm{O}_{5}$ cathode material and prediction of improved $\mathrm{Li}_{4}(\mathrm{Mn}, \mathrm{M})_{2} \mathrm{O}_{5}$ electrodes for Li-ion batteries. Science Advances 2018, 4, eaao6754.

(635) Bhandari, A.; Jindal, P.; Bhattacharya, J. Discovery of new ground state structures for $\mathrm{Li}_{4} \mathrm{Mn}_{2} \mathrm{O}_{5}$ and $\mathrm{V}_{2} \mathrm{O}_{5}$ from first principles. Computational Materials Science $\mathbf{2 0 1 9}$, $159,454-459$.

(636) Nakajima, M.; Yabuuchi, N. Lithium-Excess Cation-Disordered Rocksalt-Type Oxide 

with Nanoscale Phase Segregation: $\mathrm{Li}_{1.25} \mathrm{Nb}_{0.25} \mathrm{~V}_{0.5} \mathrm{O}_{2}$. Chemistry of Materials 2017, 29, 6927-6935.

(637) Yabuuchi, N.; Takeuchi, M.; Nakayama, M.; Shiiba, H.; Ogawa, M.; Nakayama, K.; Ohta, T.; Endo, D.; Ozaki, T.; Inamasu, T.; Sato, K.; Komaba, S. High-capacity electrode materials for rechargeable lithium batteries: $\mathrm{Li}_{3} \mathrm{NbO}_{4}$-based system with cation-disordered rocksalt structure. Proceedings of the National Academy of Sciences 2015, 112, 7650-7655.

(638) Wang, R.; Li, X.; Liu, L.; Lee, J.; Seo, D.-H.; Bo, S.-H.; Urban, A.; Ceder, G. A disordered rock-salt Li-excess cathode material with high capacity and substantial oxygen redox activity: $\mathrm{Li}_{1.25} \mathrm{Nb}_{0.25} \mathrm{Mn}_{0.5} \mathrm{O}_{2}$. Electrochemistry Communications 2015, $60,70-73$.

(639) Sharpe, R.; House, R. A.; Clarke, M. J.; Forstermann, D.; Marie, J.-J.; Cibin, G.; Zhou, K.-J.; Playford, H. Y.; Bruce, P. G.; Islam, M. S. Redox Chemistry and the Role of Trapped Molecular $\mathrm{O}_{2}$ in Li-Rich Disordered Rocksalt Oxyfluoride Cathodes. J. Am. Chem. Soc. 2020, 142, 21799-21809.

(640) House, R. A.; Jin, L.; Maitra, U.; Tsuruta, K.; Somerville, J. W.; Förstermann, D. P.; Massel, F.; Duda, L.; Roberts, M. R.; Bruce, P. G. Lithium manganese oxyfluoride as a new cathode material exhibiting oxygen redox. Energy \& Environmental Science 2018, 11, 926-932.

(641) Lun, Z; Ouyang, B.; Cai, Z.; Clément, R. J.; Kwon, D.-H.; Huang, J.; Papp, J. K.; Balasubramanian, M.; Tian, Y.; McCloskey, B. D.; Ji, H.; Kim, H.; Kitchaev, D. A.; Ceder, G. Design Principles for High-Capacity Mn-Based Cation-Disordered Rocksalt Cathodes. Chem 153-168.

(642) Chen, R.; Ren, S.; Yavuz, M.; Guda, A. A.; Shapovalov, V.; Witter, R.; Fichtner, M.; 
Hahn, $\mathrm{H} . \mathrm{Li}^{+}$intercalation in isostructural $\mathrm{Li}_{2} \mathrm{VO}_{3}$ and $\mathrm{Li}_{2} \mathrm{VO}_{2} \mathrm{~F}$ with $\mathrm{O}^{2-}$ and mixed $\mathrm{O}^{2-} / \mathrm{F}^{-}$anions. Physical Chemistry Chemical Physics 2015, 17, 17288-17295.

(643) Chen, R.; Ren, S.; Knapp, M.; Wang, D.; Witter, R.; Fichtner, M.; Hahn, H. Disordered Lithium-Rich Oxyfluoride as a Stable Host for Enhanced $\mathrm{Li}^{+}$Intercalation Storage. Advanced Energy Materials 2015, 5, 1401814.

(644) Baur, C. et al. Improved cycling stability in high-capacity bi-rich vanadium containing disordered rock salt oxyfluoride cathodes. Journal of Materials Chemistry A 2019, 7, 21244-21253.

(645) Cambaz, M. A.; Vinayan, B. P.; Pervez, S. A.; Johnsen, R. E.; Geßwein, H.; Guda, A. A.; Rusalev, Y. V.; Kinyanjui, M. K.; Kaiser, U.; Fichtner, M. Suppressing Dissolution of Vanadium from Cation-Disordered $\mathrm{Li}_{2-x} \mathrm{VO}_{2} \mathrm{~F}$ via a Concentrated Electrolyte Approach. Chemistry of Materials 2019, 31, 7941-7950.

(646) Baur, C.; Lacatusu, M.-E.; Fichtner, M.; Johnsen, R. E. Insights into Structural Transformations in the Local Structure of $\mathrm{Li}_{2} \mathrm{VO}_{2} \mathrm{~F}$ Using Operando X-ray Diffraction and Total Scattering: Amorphization and Recrystallization. ACS Applied Materials \& Interfaces 2020, 12, 27010-27016.

(647) Källquist, I.; Naylor, A. J.; Baur, C.; Chable, J.; Kullgren, J.; Fichtner, M.; Edström, K.; Brandell, D.; Hahlin, M. Degradation Mechanisms in $\mathrm{Li}_{2} \mathrm{VO}_{2} \mathrm{~F}$ Li-Rich Disordered Rock-Salt Cathodes. Chemistry of Materials 2019, 31, 6084-6096.

(648) Chang, J. H.; Baur, C.; Mba, J.-M. A.; Arčon, D.; Mali, G.; Alwast, D.; Behm, R. J.; Fichtner, M.; Vegge, T.; Lastra, J. M. G. Superoxide Formation in $\mathrm{Li}_{2} \mathrm{VO}_{2} \mathrm{~F}$ Cathode Material - a Combined Computational and Experimental Investigation of Anionic Redox Activity. Journal of Materials Chemistry A 2020, 8, 16551-16559.

649) Lee, J.; Kitchaev, D. A.; Kwon, D.-H.; Lee, C.-W.; Papp, J. K.; Liu, Y.-S.; Lun, Z.; Clément, R. J.; Shi, T.; McCloskey, B. D.; Guo, J.; Balasubramanian, M.; Ceder, G. Re- 

versible $\mathrm{Mn}^{2+} / \mathrm{Mn}^{4+}$ double redox in lithium-excess cathode materials. Nature $\mathbf{2 0 1 8}$, $556,185-190$.

(650) Freire, M.; Lebedev, O.; Maignan, A.; Jordy, C.; Pralong, V. Nanostructured $\mathrm{Li}_{2} \mathrm{MnO}_{3}$ : a disordered rock salt type structure for high energy density Li ion batteries. Journal of Materials Chemistry A 2017, 5, 21898-21902.

(651) Jacquet, Q.; Iadecola, A.; Saubanere, M.; Li, H.; Berg, E.J.; Rousse, G.; Cabana, J.; Doublet, M. L.; Tarascon, J. M. Charge Transfer Band Gap as an Indicator of Hysteresis in Li-Disordered Rock Salt Cathodes for Li-Ion Batteries. J. Am. Chem. Soc. 2019, 141, 11452-11464.

(652) Clément, R.; Lun, Z.; Ceder, G. Cation-disordered rocksalt transition metal oxides and oxyfluorides for high energy lithium-ion cathodes. Energy \& Environmental Science 2020, 13, 345-373.

(653) Nytén, A.; Abouimrane, A.; Armand, M.;Gustafsson, T.; Thomas, J. O. Electrochemical performance of $\mathrm{Li}_{2} \mathrm{FeSiO}_{4}$ as a new Li-battery cathode material. Electrochemistry communications 2005, 7, 156-160.

(654) Sirisopanaporn, C.; Dominko, R.; Masquelier, C.; Armstrong, A. R.; Mali, G.; Bruce, P. G. Polymorphism in $\mathrm{Li}_{2}(\mathrm{Fe}, \mathrm{Mn}) \mathrm{SiO}_{4}$ : A combined diffraction and NMR study. Journal of Materials Chemistry 2011, 21, 17823-17831.

(655) Islam, M. S.; Dominko, R.; Masquelier, C.; Sirisopanaporn, C.; Armstrong, A. R.; Bruce, P. G. Silicate cathodes for lithium batteries: alternatives to phosphates? Journal of Materials Chemistry 2011, 21, 9811-9818.

(656) Padhi, A.; Nanjundaswamy, K.; Masquelier, C.; Goodenough, J. Mapping of transition metal redox energies in phosphates with NASICON structure by lithium intercalation. Journal of the Electrochemical Society 1997, 144, 2581. 
(657) Tygesen, A. S.; Chang, J. H.; Vegge, T.; García-Lastra, J. M. Computational framework for a systematic investigation of anionic redox process in Li-rich compounds. $n p j$ Computational Materials 2020, 6, 1-9.

(658) Armstrong, A. R.; Bruce, P. G. Synthesis of layered $\mathrm{LiMnO}_{2}$ as an electrode for rechargeable lithium batteries. Nature 1996, 381, 499-500.

(659) Mosbah, A.; Verbaere, A.; Tournoux, M. Phases $\mathrm{Li}_{x} \mathrm{MnO}_{2}$ rattachees au type spinelle. Materials research bulletin 1983, 18, 1375-1381.

(660) Dittrich, G.; Hoppe, R. Zur Kristallstruktur von LiMnO 2 . Zeitschrift für anorganische und allgemeine Chemie 1969, 368, 262-270.

(661) Gao, P.; Ishikawa, R.; Tochigi, E.; Kumamoto, A.; Shibata, N.; Ikuhara, Y. AtomicScale Tracking of a Phase Transition from Spinel to Rocksalt in Lithium Manganese Oxide. Chemistry of Materials 2017, 29, 1006-1013.

(662) Chen, B.; Ben, L.; Yu, H.; Chen, Y.; Huang, X. Understanding Surface Structural Stabilization of the High-Temperature and High-Voltage Cycling Performance of $\mathrm{Al}^{3+}$ Modified $\mathrm{LiMn}_{2} \mathrm{O}_{4}$ Cathode Matérial. ACS Applied Materials \& Interfaces 2018, 10, 550-559.

(663) Reed, J.; Ceder, G.; Van Der Ven, A. Layered-to-spinel phase transition in $\mathrm{Li}_{x} \mathrm{MnO}_{2}$. Electrochemical and Solid State Letters 2001, 4, A78.

(664) Bruce, P. G.; Scrosati, B.; Tarascon, J.-M. Nanomaterials for rechargeable lithium batteries. Angewandte Chemie International Edition 2008, 47, 2930-2946.

(665) Franger, S.; Benoit, C.; Bourbon, C.; Le Cras, F. Chemistry and electrochemistry of composite $\mathrm{LiFePO}_{4}$ materials for secondary lithium batteries. Journal of Physics and Chemistry of Solids 2006, 67, 1338-1342. 
${ }_{4923}$ (666) Ellis, B.; Kan, W. H.; Makahnouk, W.; Nazar, L. Synthesis of nanocrystals and mor-

4924

4925

4926

4927

4928

4929

4930

4931

4932

4933

4934

4935

4936

4937

4938

4939

4940

4941

4942

4943

4944

4945 phology control of hydrothermally prepared $\mathrm{LiFePO}_{4}$. Journal of Materials Chemistry 2007, 17, 3248-3254.

(667) Malik, R.; Burch, D.; Bazant, M.; Ceder, G. Particle size dependence of the ionic diffusivity. Nano letters 2010, 10, 4123-4127.

(668) Ledwaba, R. S.; Ngoepe, P.; Sayle, D. Modeling Diffusion-Induced Stress In Li-Mn-O Nanocomposite Cathode Materials. APS March Meeting Abstracts. 2018; p E20.006.

(669) Sayle, T. X.; Maphanga, R. R.; Ngoepe, P. E.; Sayle, D. C. Predicting the electrochemical properties of $\mathrm{MnO}_{2}$ nanomaterials used in rechargeable Li batteries: simulating nanostructure at the atomistic level. Journal of the American Chemical Society 2009 , 131, 6161-6173.

(670) Gupta, P. K.; Bhandari, A.; Bhattacharya, J.; Pala, R. G. S. Correlating Voltage Profile to Molecular Transformations in Ramsdellite $\mathrm{MnO}_{2}$ and Its Implication for Polymorph Engineering of Lithium Ion Battery Cathodes. The Journal of Physical Chemistry C 2018, 122, 11689-11700.

(671) Gupta, P. K.; Bhandari, A.; Bhattacharya, J.; Ganesh S Pala, R. Higher voltage, wider voltage plateau, longer cycle life, and faster kinetics via thermally modulated interfaces between Ramsdellite and Pyrolusite $\mathrm{MnO}_{2}$ for lithium-ion battery cathodes. Journal of Power Sources 2020, 450, 227619.

(672) Moriwake, H.; Kuwabara, A.; Fisher, C. A.; Huang, R.; Hitosugi, T.; Ikuhara, Y. H.; Oki, H.; Ikuhara, Y. First-Principles Calculations of Lithium-Ion Migration at a Coherent Grain Boundary in a Cathode Material, $\mathrm{LiCoO}_{2}$. Advanced Materials 2013, 25, 618-622.

4946 (673) Dixit, M.; Engel, H.; Eitan, R.; Aurbach, D.; Levi, M. D.; Kosa, M.; Major, D. T. 
4947

4948

4949

4950

4951

4952

4953

4954

4955

4956

4957

4958

4959

4960

4961

4962

4963

4964

4965

4966

4967

4968

4969

Classical and Quantum Modeling of $\mathrm{Li}$ and $\mathrm{Na}$ Diffusion in $\mathrm{FePO}_{4}$. The Journal of Physical Chemistry C 2015, 119, 15801-15809.

(674) Morgan, D.; Van der Ven, A.; Ceder, G. Li Conductivity in $\mathrm{Li}_{x} \mathrm{MPO}_{4}$ $(\mathrm{M}=\mathrm{Mn}, \mathrm{Fe}, \mathrm{Co}, \mathrm{Ni})$ Olivine Materials. Electrochemical and Solid-State Letters 2004, 7, A30-A32.

(675) Ouyang, C.; Shi, S.; Wang, Z.; Huang, X.; Chen, L. First-principles study of Li ion diffusion in $\mathrm{LiFePO}_{4}$. Physical Review B 2004, 69, 104303.

(676) Islam, M. S.; Driscoll, D. J.; Fisher, C. A. J.; Slater, P. R. Atomic-scale investigation of defects, dopants, and lithium transport in the $\mathrm{LiFePO}_{4}$ olivine-type battery material. Chemistry of Materials 2005, 17, 5085-5092.

(677) Amin, R.; Balaya, P.; Maier, J. Anisotropy of electronic and ionic transport in $\mathrm{LiFePO}_{4}$ single crystals. Electrochemical and Solid State Letters 2006, 10, A13.

(678) Van der Ven, A.; Ceder, G. Lithium diffusion in layered $\mathrm{Li}_{x} \mathrm{CoO}_{2}$. Electrochemical and Solid State Letters 2000, 3, 301.

(679) Thackeray, M. M. Manganese oxides for lithium batteries. Progress in Solid State Chemistry 1997, 25, 1-71.

(680) Proell, J.; Kohler, R.; Mangang, A.; Ulrich, S.; Ziebert, C.; Pfleging, W. 3D structures in battery materials. Journal of Laser Micro Nanoengineering 2012, 7, 97.

(681) Nishimura, S.+i.; Kobayashi, G.; Ohoyama, K.; Kanno, R.; Yashima, M.; Yamada, A. Experimental visualization of lithium diffusion in $\mathrm{Li}_{x} \mathrm{FePO}_{4}$. Nature materials 2008, 7, $707-711$.

(682) Van der Ven, A.; Thomas, J. C.; Xu, Q.; Swoboda, B.; Morgan, D. Nondilute diffusion from first principles: Li diffusion in $\mathrm{Li}_{x} \mathrm{TiS}_{2}$. Physical Review B 2008, 78, 104306. 
4970 (683) Bhattacharya, J.; Van der Ven, A. First-principles study of competing mechanisms of

4971

4972

4973

4974

4975

4976

4977

4978

4979

4980

4981

4982

4983

4984

4985

4986

4987

4988

4989

4990

4991

4992 nondilute Li diffusion in spinel $\mathrm{Li}_{x} \mathrm{TiS}_{2}$. Physical Review B 2011, 83, 144302.

(684) Bhattacharya, J.; Van der Ven, A. Phase stability and nondilute Li diffusion in spinel $\mathrm{Li}_{1+x} \mathrm{Ti}_{2} \mathrm{O}_{4}$. Physical Review B 2010, 81, 104304.

(685) Van Der Ven, A.; Bhattacharya, J.; Belak, A. A. Understanding Li diffusion in Liintercalation compounds. Accounts of Chemical Research 2013, 46, 1216-1225.

(686) Van der Ven, A.; Ceder, G. Lithium diffusion mechanisms in layered intercalation compounds. Journal of power sources 2001, 97, 529-531.

(687) Choi, S. H.; Shlyakhtin, O.; Kim, J.; Yoon, Y. S. Structural and electrochemical properties of $\mathrm{Lil}_{1+x} \mathrm{Ni}_{0.5} \mathrm{Mn}_{0.5} \mathrm{O}_{2+\delta}(0 \leq x \leq 0.7)$ cathode materials for lithium-ion batteries. Journal of power sources 2005, 140, 355-360.

(688) Kang, K.; Meng, Y. S.; Breger, J.; Grey, C.P.; Ceder, G. Electrodes with high power and high capacity for rechargeable lithium batteries. Science 2006, 311, 977-980.

(689) Laubach, S.; Laubach, S.; Schmidt, P. C.; Gröting, M.; Albe, K.; Jaegermann, W.; Wolf, W. Structure, Electronic Structure and Defect Formation Energies of $\mathrm{Li}_{x} \mathrm{Co}_{1-y} \mathrm{Ni}_{y} \mathrm{O}_{2}$ as a Function of $x(0<x<1)$ and $y(y=0,0.5,1)$. Zeitschrift für Physikalische Chemie 2009, 223, 1327-1340.

(690) Lee, E.; Persson, K. A. Solid-Solution Li Intercalation as a Function of Cation Order/Disorder in the High-Voltage $\mathrm{Li}_{x} \mathrm{Ni}_{0.5} \mathrm{Mn}_{1.5} \mathrm{O}_{4}$ Spinel. Chemistry of Materials 2013, 25, 2885-2889.

(691) Hao, S.; Lu, Z.; Wolverton, C. Quaternary phase diagrams of spinel $\mathrm{Li}_{y} \square_{1-y} \mathrm{Mn}_{x} \mathrm{Ni}_{2-x} \mathrm{O}_{4}$ and composite cathode voltages for concentration gradient materials. Physical Review B 2016, 94, 014114. 
(692) Capsoni, D.; Bini, M.; Chiodelli, G.; Mustarelli, P.; Massarotti, V.; Azzoni, C. B.; Mozzati, M. C.; Linati, L. Inhibition of Jahn-Teller Cooperative Distortion in $\mathrm{LiMn}_{2} \mathrm{O}_{4}$ Spinel by $\mathrm{Ga}^{3+}$ Doping. The Journal of Physical Chemistry B 2002, 106, 7432-7438.

(693) Singh, G.; Gupta, S.; Prasad, R.; Auluck, S.; Gupta, R.; Sil, A. Suppression of JahnTeller distortion by chromium and magnesium doping in spinel $\mathrm{LiMn}_{2} \mathrm{O}_{4}$ : A firstprinciples study using GGA and GGA + U. Journal of Physics and Chemistry of Solids 2009, 70, 1200-1206.

(694) Padhi, A.; Nanjundaswamy, K.; Goodenough, J. Phospho-olivines as positive-electrode materials for rechargeable lithium batteries. Journal of the Electrochemical Society 1997, $144,1188-1194$.

(695) Thackeray, M. M.; David, W. I. F.; Bruce, P. G.; Goodenough, P. G. Lithium Insertion into Manganese Spinels. Materials Research Bulletin 1983, 18, 461-472.

(696) Koga, H.; Croguennec, L.; Menetrier, M.; Douhiol, K.; Belin, S.; Bourgeois, L.; Suard, E.; Weill, F. Reversible Oxygen Participation to the Redox Processes Revealed for $\mathrm{Li}_{1.20} \mathrm{Mn}_{0.54} \mathrm{Co}_{0.13} \mathrm{Ni}_{0.13} \mathrm{O}_{2}$. J. Electrochem. Soc. 2013, 160, A786-A792.

(697) Sathiya, M.; Lerich, J.-B.; Salager, E.; Gourier, D.; Tarascon, J.-M.; Vezin, H. Electron paramagnetic resonance imaging for real-time monitoring of Li-ion batteries. Nat. Commun. 2015, 6, 6276.

(698) McCalla, E.; Abakumov, A. M.; Saubanère, M.; Foix, D.; Berg, E. J.; Rousse, G.; Doublet, M.-L.; Gonbeau, D.; Novák, P.; Van Tendeloo, G., et al. Visualization of OO peroxo-like dimers in high-capacity layered oxides for Li-ion batteries. Science $\mathbf{2 0 1 5}$ $350,1516-1521$.

(699) Cao, T.; Shi, C.; Zhao, N.; He, C.; Li, J.; Liu, E. Understanding the Electrochemical Properties of Li-Rich Cathode Materials from First-Principles Calculations. The Journal of Physical Chemistry C 2015, 119, 28749-28756. 
5018 (700) Shimoda, K.; Minato, T.; Nakanishi, K.; Komatsu, H.; Matsunaga, T.; Tanida, H.;

5019

5020

5021

5022

5023

5024

5025

5026

5027

5028

5029

5030

5031

5032

5033

5034

5035

5036

5037

5038

5039

5040

5041 Arai, H.; Ukyo, Y.; Uchimoto, Y.; Ogumi, Z. Oxidation behaviour of lattice oxygen in Li-rich manganese-based layered oxide studied by hard X-ray photoelectron spectroscopy. J. Mater. Chem. A 2016, 4, 5909-5916.

(701) Chen, H.; Islam, M. S. Lithium Extraction Mechanism in Li-Rich $\mathrm{Li}_{2} \mathrm{MnO}_{3}$ Involving Oxygen Hole Formation and Dimerization. Chemistry of Materials 2016, 28, 66566663.

(702) Luo, K.; Roberts, M. R.; Hao, R.; Guerrini, N.; Pickup, D. M.; Liu, Y.-S.; Edström, K.; Guo, J.; Chadwick, A. V.; Duda, L. C.; Bruce, P. G. Charge-compensation in 3dtransition-metal-oxide intercalation cathodes through the generation of localized electron holes on oxygen. Nature Chemistry 2016, 8, 684-691.

(703) Muhammad, S.; Kim, H.; Kim, Y.; Kim, D; Song, J. H.; Yoon, J.; Park, J.-H.; Ahn, S.-J.; Kang, S.-H.; Thackeray, M. M.; Yoon, W.-S. Evidence of reversible oxygen participation in anomalously high capacity Li- and Mn-rich cathodes for Li-ion batteries. Nano Energy 2016, 21, 172-184.

(704) Zhan, C. et al. Enabling the high capacity of lithium-rich anti-fluorite lithium iron oxide by simultaneous anionic and cationic redox. Nature Energy 2017, 2, 963-971.

(705) Zheng, J.; Myeong, S.; Cho, W.; Yan, P.; Xiao, J.; Wang, C.; Cho, J.; Zhang, J. G. Liand Mn-Rich Cathode Materials: Challenges to Commercialization. 2017, 7, 1601284.

(706) Ben Yahia, M.; Vergnet, J.; Saubanère, M.; Doublet, M.-L. Unified picture of anionic redox in Li/Na-ion batteries. Nature Materials 2019, 18, 496-502.

(707) Hua, W.; Wang, S.; Knapp, M.; Leake, S. J.; Senyshyn, A.; Richter, C.; Yavuz, M.; Binder, J. R.; Grey, C. P.; Ehrenberg, H.; Indris, S.; Schwarz, B. Structural insights into the formation and voltage degradation of lithium- and manganese-rich layered $5042 \quad$ oxides. Nature Communications 2019, 10, 5365. 
${ }_{5043}$ (708) Li, L.; Castro, F. C.; Park, J. S.; Li, H.; Lee, E.; Boyko, T. D.; Freeland, Z., J.

5044

5045

5046

5047

5048

5049

5050

5051

5052

5053

5054

5055

5056

5057

5058

5059

5060

5061

5062

5063

5064

5065

5066

5067 W.and Yao; Fister, T. T.; Vinson, J.; Shirley, E. L.; Wolverton, C.; Cabana, J.; Dravid, V. P.; M., T. M.; Chan, M. K. Y. Probing Electrochemically Induced Structural Evolution and Oxygen Redox Reactions in Layered Lithium Iridate. Chem. Mater. 2019, 31, 4341-4352.

(709) Eum, D.; Kim, B.; Kim, S. J.; Park, H.; Wu, J.; Cho, S.-P.; Yoon, G.; Lee, M. H.; Jung, S.-K.; Yang, W., et al. Voltage decay and redox asymmetry mitigation by reversible cation migration in lithium-rich layered oxide electrodes. Nature materials 2020, 19, 419-427.

(710) Gent, W. E.; Abate, I. I.; Yang, W.; Nazar, L. F.; Chueh, W. C. Design Rules for High-Valent Redox in Intercalation Electrodes. 2020, 4, 1369-1397.

(711) Sathiya, M.; Ramesha, K.; Rousse, G.; Foix, D.; Gonbeau, D.; Prakash, A. S.; Doublet, M. L.; Hemalatha, K.; Tarascon, J. M. High performance $\mathrm{Li}_{2} \mathrm{Ru}_{1-y} \mathrm{Mn}_{y} \mathrm{O}_{3}(0.2 \leq$ $\mathrm{y} \leq 0.8)$ cathode materials for rechargeable lithium-ion batteries: Their understanding. Chemistry of Materials 2013, 25, 1121-1131.

(712) Xiao, R.; Li, H.; Chen, L. Density functional investigation on $\mathrm{Li}_{2} \mathrm{MnO}_{3}$. Chemistry of Materials 2012, 24, 4242-4251.

(713) Hong, J. et al. Metal-oxygen decoordination stabilizes anion redox in Li-rich oxides. Nature Materials 2019, 18, 256-265.

(714) Davies, D. W.; Morgan, B. J.; Scanlon, D. O.; Walsh, A. Low-cost descriptors of electrostatic and electronic contributions to anion redox activity in batteries. IOP SciNotes 2020, 1, 024805.

(715) Xiao, Y.; Liu, T.; Liu, J.; He, L.; Chen, J.; Zhang, J.; Luo, P.; Lu, H.; Wang, R.; Zhu, W., et al. Insight into the origin of lithium/nickel ions exchange in layered Li $\left(\mathrm{Ni}_{x} \mathrm{Mn}_{y} \mathrm{Co}_{z}\right) \mathrm{O}_{2}$ cathode materials. Nano Energy 2018, 49, 77-85. 
(716) Sun, H.; Zhao, K. Electronic Structure and Comparative Properties of $\mathrm{LiNi}_{x} \mathrm{Mn}_{y} \mathrm{Co}_{z} \mathrm{O}_{2}$ Cathode Materials. The Journal of Physical Chemistry C 2017, 121, 6002-6010.

(717) Dixit, M.; Markovsky, B.; Schipper, F.; Aurbach, D.; Major, D. T. Origin of structural degradation during cycling and low thermal stability of Ni-rich layered transition metal-based electrode materials. The Journal of Physical Chemistry $C$ 2017, 121, $22628-22636$.

(718) Hoang, K.; Johannes, M. Defect physics and chemistry in layered mixed transition metal oxide cathode materials:(Ni, $\mathrm{Co}, \mathrm{Mn})$ vs (Ni, Co, Al). Chemistry of Materials 2016, 28, 1325-1334.

(719) Dixit, M.; Markovsky, B.; Aurbach, D.; Major, D. T. Unraveling the effects of Al doping on the electrochemical properties of $\mathrm{LiNi}_{0.5} \mathrm{Co}_{0.2} \mathrm{Mn}_{0.3} \mathrm{O}_{2}$ using first principles. Journal of The Electrochemical Society 2017, 164, A6359.

(720) Lu, Z.; Donaberger, R.; Dahn, J. Superlattice ordering of Mn, Ni, and Co in layered alkali transition metal oxides with P2, P3, and O3 structures. Chemistry of materials 2000, 12, 3583-3590.

(721) Du, T.; Xu, B.; Wu, M.; Liu, G.; Ouyang, C. Insight into the vibrational and thermodynamic properties of layered lithium transition-metal oxides $\mathrm{LiMO}_{2}(\mathrm{M}=\mathrm{Co}, \mathrm{Ni}$, Mn): a first-principles study. The Journal of Physical Chemistry C 2016, 120, 58765882.

(722) Shang, S.; Wang, Y.; Mei, Z.; Hui, X.; Liu, Z. Lattice dynamics, thermodynamics, and bonding strength of lithium-ion battery materials $\mathrm{LiMPO}_{4}(\mathrm{M}=\mathrm{Mn}, \mathrm{Fe}, \mathrm{Co}$, and $\mathrm{Ni}$ : a comparative first-principles study. Journal of Materials Chemistry 2012, 22, $1142-1149$. 5092 conductivity: A first-principles study. Physical Review Materials 2019, 3, 035405. 
5093

5094

5095

5096

5097

5098

5099

5100

5101

5102

5103

5104

5105

5106

5107

5108

5109

5110

5111

5112

5113

5114

5115

5116

(732) Xu, B.; Fell, C. R.; Chi, M.; Meng, Y. S. Identifying surface structural changes in layered Li-excess nickel manganese oxides in high voltage lithium ion batteries: A thermal conductivity: A comparison of molecular dynamics and Boltzmann transport approaches. Physical Review Materials 2019, 3, 085401.

(725) Mattila, N.; Karttunen, A. J. Lattice thermal conductivity of $\mathrm{NaCoO}_{2}$ and $\mathrm{LiCoO}_{2}$ intercalation materials studied by hybrid density functional theory. Materials Research Express 2020, 7, 075502.

(726) Feng, T.; O'Hara, A.; Pantelides, S. T. Quantum prediction of ultra-low thermal conductivity in lithium intercalation materials. Naño Energy 2020, 104916.

(727) Qian, X.; Gu, X.; Dresselhaus, M. S.; Yang, R. Anisotropic tuning of graphite thermal conductivity by lithium intercalation. The Journal of Physical Chemistry Letters 2016, 7, 4744-4750.

(728) Wei, Z.; Yang, F.; Bi, K.; Yang, J.; Chen, Y. Tunable Anisotropic Thermal Conductivity and Elastic Properties in Intercalated Graphite via Lithium Ions. The Journal of Physical Chemistry C 2018, 122, 1447-1455.

(729) Xia, Y.; Hegde, V. I.; Pal, K.; Hual, X.; Gaines, D.; Patel, S.; He, J.; Aykol, M.; Wolverton, C. High-Throughput Study of Lattice Thermal Conductivity in Binary Rocksalt and Zinc Blende Compounds Including Higher-Order Anharmonicity. Physical Review $X 2020,10,041029$.

(730) Zhang, Q.; Uchaker, E.; Candelaria, S. L.; Cao, G. Nanomaterials for energy conversion and storage. Chemical Society Reviews 2013, 42, 3127-3171.

(731) Kramer, D.; Ceder, G. Tailoring the morphology of $\mathrm{LiCoO}_{2}$ : a first principles study. Chemistry of Materials 2009, 21, 3799-3809.

(724) Puligheddu, M.; Xia, Y.; Chan, M.; Galli, G. Computational prediction of lattice 
$5117 \quad$ joint experimental and theoretical study. Energy $\&$ Environmental Science 2011, 4, $5118 \quad 2223-2233$.

5119 (733) Dahéron, L.; Martinez, H.; Dedryvere, R.; Baraille, I.; Ménétrier, M.; Denage, C.; 5120 Delmas, C; Gonbeau, D. Surface properties of $\mathrm{LiCoO}_{2}$ investigated by XPS analyses 5121 5122

5123 5124 5125 5126 5127 5128 5129 5130 5131 5132 5133 5134 5135 5136 5137 5138 5139 (2) and theoretical calculations. The Journal of Physical Chemistry C 2009, 113, 58435852.

(734) Kim, Y.; Lee, H.; Kang, S. First-principles and experimental investigation of the morphology of layer-structured $\mathrm{LiNiO}_{2}$ and $\mathrm{LiCoO}_{2}$. Journal of Materials Chemistry 2012, 22, 12874-12881.

(735) Benedek, R.; Thackeray, M. Simulation of the surface structure of lithium manganese oxide spinel. Physical Review B 2011, 83, 195439.

(736) Karim, A.; Fosse, S.; Persson, K. A. Surface structure and equilibrium particle shape of the $\mathrm{LiMn}_{2} \mathrm{O}_{4}$ spinel from first-principles calculations. Physical Review B 2013, 87, 075322 .

(737) Leung, K. First-principles modeling of the initial stages of organic solvent decomposition on $\mathrm{Li}_{x} \mathrm{Mn}_{2} \mathrm{O}_{4}$ (100) surfaces. The Journal of Physical Chemistry C 2012, 116, $9852-9861$.

(738) Tompsett, D. A.; Parker, S. C.; Bruce, P. G.; Islam, M. S. Nanostructuring of $\beta-\mathrm{MnO}_{2}$ : The important role of surface to bulk ion migration. Chemistry of materials 2013, 25, $536-541$.

(739) Qian, D.; Hinuma, Y.; Chen, H.; Du, L.-S.; Carroll, K. J.; Ceder, G.; Grey, C. P.; Meng, Y. S. Electronic spin transition in nanosize stoichiometric lithium cobalt oxide. Journal of the American Chemical Society 2012, 134, 6096-6099. 
5140 (740) Hong, L.; Hu, L.; Freeland, J. W.; Cabana, J.; Öğüt, S.; Klie, R. F. Electronjc Structure of $\mathrm{LiCoO}_{2}$ Surfaces and Effect of Al Substitution. The Journal of Physical Chemistry C 2019, 123, 8851-8858.

5143

5144

5145

5146

5147

5148

5149

5150

5151

5152

5153

5154

5155

5156

5157

5158

5159

5160

5161

5162

5163

5164

(741) Abbaspour-Tamijani, A.; Bennett, J. W.; Jones, D. T.; Cartagena-Gonzalez, N.; Jones, Z. R.; Laudadio, E. D.; Hamers, R. J.; Santana, J. A.; Mason, S. E. DFT and thermodynamics calculations of surface cation release in $\mathrm{LiCoO}_{2}$. Applied Surface Science 2020, 515, 145865.

(742) Deng, Y.-P.; Yin, Z.-W.; Wu, Z.-G.; Zhang, S.-J.; Fu, F.; Zhang, T.; Li, J.T.; Huang, L.; Sun, S.-G. Layered/spinel heterostructured and hierarchical micro/nanostructured Li-rich cathode materials with enhanced electrochemical properties for Li-ion batteries. ACS applied materials \&f interfaces 2017, 9, 21065-21070.

(743) Deng, Y.-P.; Fu, F.; Wu, Z.-G.; Yin, Z.-W.; Zhang, T.; Li, J.-T.; Huang, L.; Sun, S.-G. Layered/spinel heterostructured Li-rich materials synthesized by a one-step solvothermal strategy with enhanced electrochemical performance for Li-ion batteries. Journal of Materials Chemistry A 2016, 4, 257-263.

(744) Long, B. R.; Croy, J. R.; Park, J. S.; Wen, J.; Miller, D. J.; Thackeray, M. M. Advances in stabilizing 'layered-layered' $\mathrm{xLi}_{2} \mathrm{MnO}_{3} \cdot(1-\mathrm{x}) \mathrm{LiMO}_{2}(\mathrm{M}=\mathrm{Mn}, \mathrm{Ni}, \mathrm{Co})$ electrodes with a spinel component. Journal of the Electrochemical Society 2014, 161, A2160.

(745) Wang, D.; Belharouak, I.; Zhou, G.; Amine, K. Nanoarchitecture multi-structural cathode materials for high capacity lithium batteries. Advanced Functional Materials 2013, 23, 1070-1075.

(746) Warburton, R. E.; Castro, F. C.; Deshpande, S.; Madsen, K. E.; Bassett, K. L.; Dos Reis, R.; Gewirth, A. A.; Dravid, V. P.; Greeley, J. Oriented $\mathrm{LiMn}_{2} \mathrm{O}_{4}$ Particle Fracture from Delithiation-Driven Surface Stress. ACS applied materials $\&$ interfaces 2020, 12, 49182-49191. 
5165 (747) Liang, C.; Longo, R. C.; Kong, F.; Zhang, C.; Nie, Y.; Zheng, Y.; Cho, K. Ab

5166

5167

5168

5169

5170

5171

5172

5173

5174

5175

5176

5177

5178

5179

5180

5181

5182

5183

5184

5185

5186

5187

5188

(755) Bhandari, A.; Bhattacharya, J.; Pala, R. G. S. Adsorption Preference of HF over Ethylene Carbonate Leads to Dominant Presence of Fluoride Products in $\mathrm{LiFePO}_{4}$ $(\mathrm{NCM})(\mathrm{y} \leq 0.1)$ Cathodes. ACS applied materials \& interfaces 2018, 10, 6673-6680.

(748) Chen, G.; Song, X.; Richardson, T. J. Electron microscopy study of the LiFePO 4 to $\mathrm{FePO}_{4}$ phase transition. Electrochemical and Solid State Letters 2006, 9, A295.

(749) Islam, M. S. Recent atomistic modelling studies of energy materials: batteries included. Philosophical Transactions of the Royal Society A: Mathematical, Physical and Engineering Sciences 2010, 368, 3255-3267.

(750) Tankhilsaikhan, B.; Gorfman, S.; Tungalag, N.; Tuvjargal, N.; Davaasambuu, J.; Pietsch, U. Solid State Phenomena; 2019; Vol. 288; pp 98-103.

(751) Xu, G.; Zhong, K.; Yang, Y.; Zhang, J.-M.; Huang, Z. Insight into delithiation process on the $\mathrm{LiFePO}_{4}$ (010) surface from a novel viewpoint of the work function. Solid State Ionics 2019, 338, 25-30.

(752) Zhang, Y.; Alarco, J. A.; Nerkar, J. Y.; Best, A. S.; Snook, G. A.; Talbot, P. C.; Cowie, B. C. Observation of preferential cation doping on the surface of $\mathrm{LiFePO}_{4}$ particles and its effect on properties. ACS Applied Energy Materials 2020, 3, 91589167.

(753) Li, Y.; Chen, H.; Lim, K.; Deng, H. D.; Lim, J.; Fraggedakis, D.; Attia, P. M.; Lee, S. C.; Jin, N.; Moškon, J., et al. Fluid-enhanced surface diffusion controls intraparticle phase transformations. Nature materials 2018, 17, 915-922.

(754) Edström, K.; Gustafsson, T.; Thomas, J. O. The cathode-electrolyte interface in the Li-ion battery. Electrochimica Acta 2004, 50, 397-403.

Initio Study on Surface Segregation and Anisotropy of Ni-Rich $\mathrm{LiNi}_{1-2 y} \mathrm{Co}_{y} \mathrm{Mn}_{y} \mathrm{O}_{2}$ 
Cathode-Electrolyte Interface in Li-Ion Batteries. The Journal of Physical Chemistry C 2020, 124, 9170-9177.

5191

5192

5193

5194

5195

5196

5197

5198

5199

5200

5201

5202

5203

5204

5205

5206

5207

5208

5209

5210

5211

5212

(762) Dathar, G. K. P.; Sheppard, D.; Stevenson, K. J.; Henkelman, G. Calculations of

(756) Tebbe, J. L.; Fuerst, T. F.; Musgrave, C. B. Mechanism of hydrofluoric acid formation in ethylene carbonate electrolytes with fluorine salt additives. Journal of Power Sources 2015, 297, 427-435.

(757) Tebbe, J. L.; Holder, A. M.; Musgrave, C. B. Mechanisms of $\mathrm{LiCoO}_{2}$ Cathode Degradation by Reaction with HF and Protection by Thin Oxide Coatings. ACS Applied Materials and Interfaces 2015, 7, 24265-24278.

(758) Bhandari, A.; Gupta, P. K.; Bhattacharya, J.; Pala, R. G. S. Higher Energy Barrier for Interfacial Li-Ion Transfer from EC/LiPF 6 Electrolyte into (010) $\mathrm{LiFePO}_{4}$ Cathode Surface than Bulk Li-Ion Diffusion within Both Cathode and Electrolyte. Journal of The Electrochemical Society 2019, 166, A2966-A2972.

(759) Tebbe, J. L.; Fuerst, T. F.; Musgrave, C. B. Degradation of Ethylene Carbonate Electrolytes of Lithium Ion Batteries yia Ring Opening Activated by $\mathrm{LiCoO}_{2}$ Cathode Surfaces and Electrolyte Species. ACS Applied Materials and Interfaces 2016, 8, 26664-26674.

(760) Xu, S.; Luo, G.; Jacobs, R.; Fang, S.; Mahanthappa, M. K.; Hamers, R. J.; Morgan, D. Ab Initio Modeling of Electrolyte Molecule Ethylene Carbonate Decomposition Reaction on $\mathrm{Li}(\mathrm{Ni}, \mathrm{Mn}, \mathrm{Co}) \mathrm{O}_{2}$ Cathode Surface. ACS Applied Materials \& Interfaces 2017, 9, 20545-20553.

(761) Malmgren, S.; Rensmo, H.; Gustafsson, T.; Gorgoi, M.; Edström, K. Non-destructive depth profiling of the solid electrolyte interphase on $\mathrm{LiFePO}_{4}$ and graphite electrodes. ECS Transactions 2010, 25, 201-210. 5213 Li-Ion Diffusion in Olivine Phosphates. Chemistry of Materials 2011, 23, 4032-4037. 
5214

5215

5216

5217

5218

5219

5220

5221

5222

5223

5224

5225

5226

5227

5228

5229

5230

5231

5232

5233

5234

5235

5236

5237

(763) Li, W.; Erickson, E. M.; Manthiram, A. High-nickel layered oxide cathodes for lithiumbased automotive batteries. Nature Energy 2020, 5, 26-34.

(764) Li, W.; Dolocan, A.; Oh, P.; Celio, H.; Park, S.; Cho, J.; Manthiram, A. Dynamic behaviour of interphases and its implication on high-energy-density cathode materials in lithium-ion batteries. Nature communications 2017, 8, 1-10.

(765) Li, W.; Liu, X.; Celio, H.; Smith, P.; Dolocan, A.; Chi, M.; Manthiram, A. Mn versus $\mathrm{Al}$ in layered oxide cathodes in lithium-ion batteries: a comprehensive evaluation on long-term cyclability. Advanced Energy Materials 2018, 8, 1703154.

(766) Middlemiss, L. A.; Rennie, A. J.; Sayers, R.; West, A. R. Characterisation of batteries by electrochemical impedance spectroscopy. Energy Reports 2020, 6, 232-241.

(767) Li, J.; Murphy, E.; Winnick, J.; Kohl, P. Studies on the cycle life of commercial lithium ion batteries during rapid charge-discharge cycling. Journal of Power Sources 2001, 102, 294-301.

(768) Song, J.; Lee, H.; Wang, Y.; Wan, C. Two-and three-electrode impedance spectroscopy of lithium-ion batteries. Journal of Power Sources 2002, 111, 255-267.

(769) McTurk, E.; Birkl, C. R.; Roberts, M. R.; Howey, D. A.; Bruce, P. G. Minimally Invasive Insertion of Reference Electrodes into Commercial Lithium-Ion Pouch Cells. ECS Electrochemistry Letters 2015, 4, A145-A147.

(770) Belt, J. R.; Bernardi, D. M.; Utgikar, V. Development and Use of a Lithium-Metal Reference Electrode in Aging Studies of Lithium-Ion Batteries. Journal of The Electrochemical Society 2014, 161, A1116-A1126.

(771) Li, J.; Manthiram, A. A Comprehensive Analysis of the Interphasial and Structural Evolution over Long-Term Cycling of Ultrahigh-Nickel Cathodes in Lithium-Ion Bat- 
5238

5239

5240

5241

5242

5243

5244

5245

5246

5247

5248

5249

5250

5251

5252

5253

5254

5255

5256

5257

5258

5259

5260

5261

5262

(772) Kim, J.-S.; Johnson, C. S.; Vaughey, J. T.; Thackeray, M. M.; Hackney, S. A.;

Yoon, W.; Grey, C. P. Electrochemical and Structural Properties of $x \operatorname{Li}_{2} \mathbf{M}^{\prime} \mathrm{O}_{3} \cdot(1-$ $x) \mathrm{LiMn}_{0.5} \mathrm{Ni}_{0.5} \mathrm{O}_{2}$ Electrodes for Lithium Batteries $\left(\mathrm{M}^{\prime}=\mathrm{Ti}, \mathrm{Mn}, \mathrm{Zr} ; 0 \leq x \leq 0.3\right)$. Chemistry of Materials 2004, 16, 1996-2006.

(773) Armstrong, A. R.; Holzapfel, M.; Novák, P.; Johnson, C. S.; Kang, S.-H.; Thackeray, M. M.; Bruce, P. G. Demonstrating oxygen loss and associated structural reorganization in the lithium battery cathode $\mathrm{Li}\left[\mathrm{Ni}_{0.2} \mathrm{Li}_{0.2} \mathrm{Mn}_{0.6}\right] \mathrm{O}_{2}$. Journal of the American Chemical Society 2006, 128, 8694-8698.

(774) Bhandari, A.; Bhattacharya, J. Review-Manganese Dissolution from Spinel Cathode: Few Unanswered Questions. Journal of The Electrochemical Society 2016, 164, A106A127.

(775) Nakayama, K.; Ishikawa, R.; Kobayashi, S.; Shibata, N.; Ikuhara, Y. Dislocation and oxygen-release driven delithiation in $\mathrm{Li}_{2} \mathrm{MnO}_{3}$. Nature Communications 2020, 11, 4452.

(776) Deringer, V. L.; Caro, M. A.; Csányi, G. Machine learning interatomic potentials as emerging tools for materials science. Advanced Materials 2019, 31, 1902765.

(777) Assat, G.; Glazier, S. L.; Delacourt, C.; Tarascon, J.-M. Probing the thermal effects of voltage hysteresis in anionic redox-based lithium-rich cathodes using isothermal calorimetry. Nature Energy 2019, 4, 647-656.

(778) Grimsmann, F.; Brauchle, F.; Gerbert, T.; Gruhle, A.; Knipper, M.; Parisi, J. Hysteresis and current dependence of the thickness change of lithium-ion cells with graphite anode. Journal of Energy Storage 2017, 12, 132-137.

(779) Zheng, T.; McKinnon, W. R.; Dahn, J. R. Hysteresis during Lithium Insertion in Hydrogen-Containing Carbons. Journal of The Electrochemical Society 1996, 143, 
${ }_{5263}$ (780) Edge, J. S.; O’Kane, S.; Prosser, R.; Kirkaldy, N. D.; Patel, A. N.; Hales, A.; Ghosh, A.;

5264

5265

5266

5267

5268

5269

5270

5271

5272

5273

5274

5275

5276

5277

5278

5279

5280

5281

46
47
48
49
50
51
52
53
54
55
56
57
58
59
60
Ai, W.; Chen, J.; Jiang, J., et al. Lithium ion battery degradation: what you need to know. Physical Chemistry Chemical Physics 2021, 23, 8200-8221.

(781) Birkl, C. R.; Roberts, M. R.; McTurk, E.; Bruce, P. G.; Howey, D. A. Degradation diagnostics for lithium ion cells. Journal of Power Sources 2017, 341, 373-386.

(782) Zúlke, A.; Li, Y.; Keil, P.; Burrell, R.; Belaisch, S.; Nagarathinam, M.; Mercer, M. P.; Hoster, H. E. High-Energy Nickel-Cobalt-Aluminium Oxide (NCA) Cells on Idle: Anode- versus Cathode-Driven Side Reactions. Batteries \& Supercaps 2021, 4, 934947.

(783) Symington, A. R.; Molinari, M.; Dawson, J. A.; Statham, J. M.; Purton, J.; Canepa, P.; Parker, S. C. Elucidating the nature of grain boundary resistance in lithium lanthanum titanate. Journal of Materials Chemistry A 2021, 9, 6487-6498.

(784) Vicent-Luna, J. M.; Ortiz-Roldan, J. M.; Hamad, S.; Tena-Zaera, R.; Calero, S.; Anta, J. A. Quantum and classical molecular dynamics of ionic liquid electrolytes for $\mathrm{Na} /$ Li-based batteries: molecular origins of the conductivity behavior. ChemPhysChem 2016, 1\%, 2473-2481.

(785) Choi, Y.-S.; Park, J.-H.; Ahn, J.-P.; Lee, J.-C. Interfacial reactions in the Li/Si diffusion couples: origin of anisotropic lithiation of crystalline Si in Li-Si batteries. Scientific reports $2017, \%, 1-9$. 